\title{
Chiral Phosphoramide Catalyzed Aldol Additions of Ketone Trichlorosilyl Enolates. Mechanistic Aspects
}

\author{
Scott E. Denmark, * Son M. Pham, Robert A. Stavenger, Xiping Su, Ken-Tsung Wong and \\ Yutaka Nishigaichi
}

\section{SUPPORTING INFORMATION}

Department of Chemistry, Roger Adams Laboratory, University of Illinois, Urbana, IL 61801

Table of Contents

Page

General Experimental

S1-S3

Preparation of Phosphoramide Promoters

S3-S12

Catalyzed Aldol Additions of Trichlorosilyl Enolates

S43-S50

Non-Linear Effect Studies

S50-51

Benzoic Acid Effects

S52

Kinetics: General Procedures

S53-S55

Raw Kinetic Data (GC, ReactIR, RINMR)

S56-107

Natural Abundance ${ }^{12} \mathrm{C} /{ }^{13} \mathrm{C}$ Isotope Effects

S108-S109

References

S110

\section{General Experimental}

${ }^{1} \mathrm{H}$ NMR spectra and ${ }^{13} \mathrm{C}$ NMR spectra were recorded at $500 \mathrm{MHz}$ and $125 \mathrm{MHz}$, respectively. Spectra are referenced to residual chloroform $\left(\delta 7.26,{ }^{1} \mathrm{H} ; \delta 77.0,{ }^{13} \mathrm{C}\right)$. Chemical shifts are reported in ppm ( $\delta$ ); multiplicities are indicated by s (singlet), d (doublet), $\mathrm{t}$ (triplet), $\mathrm{q}$ (quartet), quint (quintet), sext (sextet), m (multiplet) and br (broad). Coupling constants, $J$, are reported in Hertz. ${ }^{1} \mathrm{H}$ and ${ }^{13} \mathrm{C}$ NMR assignments are corroborated by $2 \mathrm{D}$ experiments (HETCOR and COSY). Purity criteria for new compounds was established by microanalysis where possible. Otherwise, HRMS was obtained for elemental formula and P-31 NMR (>60/1 S/N) H-1 NMR $(>100 / 1 \mathrm{~S} / \mathrm{N})$ data for purity $(>98 \%)$. This criterion also applies to all the products of allylation which are known compounds (purity also assured by SFC and GC analysis). Spectra 
are available on request from denmark@scs.uiuc.edu.

Mass spectrometric data are reported in the form of $\mathrm{m} / \mathrm{z}$ (intensity relative to base peak = 100). Infrared spectra (IR) are reported in $\mathrm{cm}^{-1}$ with the indicated relative intensities: br (broad); s (strong, 67-100\%); m (medium, 34-66\%); w (weak, 0-33\%). Optical rotations are reported as follows: $[\alpha]_{D}{ }^{T}$ temperature $(\mathrm{T})$, concentration $(\mathrm{c}=\mathrm{g} / 100 \mathrm{~mL})$ and solvent. Analytical supercritical fluid chromatography (SFC) was performed on packed-column SFC with a built-in photometric detector $(\lambda=220 \mathrm{~nm})$ using a Daicel Chiralpak AD, AS, OD or OJ column, as indicated. Kugelrohr (bulb-to-bulb) distillation boiling points (bp) correspond to uncorrected airbath temperatures $(\mathrm{ABT})$. All temperatures correspond to internal reaction temperatures measured by Teflon-coated thermocouples unless otherwise noted.

${ }^{1} \mathrm{H}$ NMR spectra for all kinetic runs were recorded at $500 \mathrm{MHz}$ using a $5 \mathrm{~mm}{ }^{15} \mathrm{~N}-{ }^{31} \mathrm{P}$ broad band $(\mathrm{BB})$ probe, in methylene chloride- $d_{2}$ unless otherwise stated. Tredicator temperatures were calibrated using standard methods involving ethylene glycol and methanol as needed. Analytical gas chromatography (GC) was performed using a flame ionization detector and a Hewlett-Packard HP-5 (50 meter) column or a Hewlett-Packard Ultra-2 (50 meter) column. The injector temperature was $225^{\circ} \mathrm{C}$ (set to $160{ }^{\circ} \mathrm{C}$ for all kinetic experiments). The detector temperature was $300{ }^{\circ} \mathrm{C}$ with a split ratio of approximately 100:1.

All reactions were performed using oven $\left(140^{\circ} \mathrm{C}\right)$ and/or flame-dried glassware under an atmosphere of dry nitrogen, unless otherwise stated. Solvents and commercial reagents were purified using established procedures prior to use, with the exception of methylene chloride- $d_{2}$, which was used directly from the ampoule.

Fourier transform IR analysis was performed using a ReactIRTM 1000 fitted with a 5/8" DiComp ${ }^{\mathrm{TM}}$ Probe. Mettler-Toledo AutoChem Inc., 7075 Samuel Morse Drive, Columbia, MD, 21046 USA, or visit http://us.mt.com. Acquisitions were recorded using software version 2.1a. Small-volume, flat-bottomed reaction vessels fitted with multiple side arms were designed to mount tightly around the IR probe. In this manner, the mounted reaction vessel could be carefully flame-dried under vacuum and purged with an inert gas, in our case dry nitrogen. 1,2Dichloroethane was then carefully introduced via syringe. The appropriate background scans were taken following thermal equilibration.

Rapid Injection NMR (RINMR) analysis was performed on a home-built apparatus. For details contact denmark@scs.uiuc.edu. Samples were prepared for RINMR analysis in the 
following manner. A carefully dried NMR tube was charged with the desired amount of catalyst and fitted with a small septum. The tube was then placed under vacuum and purged several times with dry nitrogen. Dichloromethane- $d_{2}$ was then added via syringe followed by 4 . The NMR tube was quickly vortexed to ensure homogeneity and placed into a short Dewar flask at $-78^{\circ} \mathrm{C}$. Several tubes could be prepared and stored in this manner to allow sequential collection of the desired kinetic data. The septum is then removed from the cooled NMR tube and it is quickly lowered into a precooled NMR magnet. The RINMR injection probe is then carefully lowered into the open, spinning NMR tube and the entire apparatus is allowed to equilibrate for several minutes. After equilibration, the desired amount of benzaldehyde in $\mathrm{CDCl}_{3}$ is rapidly injected and is followed immediately by data collection.

Natural abundance ${ }^{13} \mathrm{C}$ NMR experiments were performed at $500 \mathrm{MHz}$ spectrometer. To obtain the highest quality NMR data, the $90^{\circ}$ pulse width (pw90) was determined by measuring the $360^{\circ}$ pulse width and dividing by 4 . Using this value, $\mathrm{T} 1$ was accurately measured and the NMR delay (d1) was set to $8 \times$ the longest T1. To assure an integration accuracy of $\leq 1 \%$, a sufficient number of transients were collected to achieve a signal-to-noise of not less than 250:1 as measured using the shortest peak. Integration was standardized by selecting a region $20 \times$ the peak width at half-height, centered about the peak in question.

\section{Literature Preparations. The following compounds were prepared following the literature methods.}

$(S, S)$ - and $(R, R)-1,2-D i p h e n y l-1,2-e t h a n e d i a m i n e,{ }^{1}(S, S)-N, N^{\prime}$-dimethyl-1,2-diphenyl-1,2ethanediamine and all other chiral diamines not listed, ${ }^{2}(S, S)-N, N^{\prime}$-diethyl-1,2-diphenyl-1,2ethanediamine, ${ }^{3}(R, R)$ - and $(S, S)-N, N^{\prime}$-1,2-tetraphenylethylene diamine, ${ }^{4}$ 1-piperidinophosphoric dichloride, ${ }^{5}$ 1-pyrrolidinylphosphoramic dichloride, ${ }^{5}$ 1-piperidinophosphorus dichloride, ${ }^{5}$ di-isopropylaminophosphorus dichloride, ${ }^{5}$ diphenylaminophosphamic dichloride. ${ }^{6}$ 


\section{Procedures.}

Preparation of Phosphoramide Promoters.

Representative Procedure I: Synthesis of Phosphoramides from Aminophosphoryl

Dichlorides. (4S,5S)-(+)-1,3-Dimethyl-4,5-diphenyl-2-piperidino-1,3,2-diazaphospholidine

2-Oxide $((S, S)-7 \mathbf{a})$

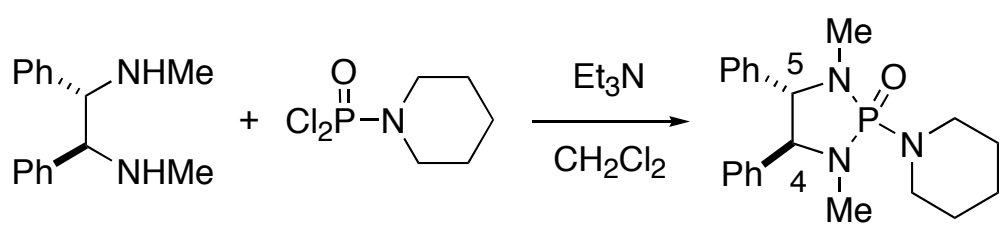

$(S, S)-18$

$(S, S)-7 \mathbf{a}$

To a solution of $(S, S)-N, N^{\prime}$-dimethyl-1,2-diphenyl-1,2-ethanediamine $(S, S)-\mathbf{1 8}^{2}$ (3.53 g, $14.7 \mathrm{mmol})$ and triethylamine $\left(5.2 \mathrm{~mL}, 37.1 \mathrm{mmol}, 2.5\right.$ equiv) in $\mathrm{CH}_{2} \mathrm{Cl}_{2}(300 \mathrm{~mL})$ was added $N$-piperidinophosphoric dichloride $(2.4 \mathrm{~mL}, 15.6 \mathrm{mmol}, 1.05$ equiv). The solution was heated to reflux under nitrogen for $44 \mathrm{~h}$ and then was cooled to rt. The solution was washed with $5 \%$ aq. $\mathrm{HCl}(2 \mathrm{X} 50 \mathrm{~mL})$ and sat. aq. $\mathrm{NaHCO}_{3}$ solution $(60 \mathrm{~mL})$ and was dried $\left(\mathrm{Na}_{2} \mathrm{SO}_{4}\right)$, filtered and concentrated on a rotavap. The residue was purified by column chromatography $\left(\mathrm{SiO}_{2}\right.$, EtOAc/i-PrOH, 19/1) and was recrystallized from hexane to afford $3.15 \mathrm{~g}(58 \%)$ of $(S, S)-7 \mathbf{a}$ as white needles.

Analytical Data for $(S, S)-7 \mathbf{a}$ :

mp: $\quad 110-111^{\circ} \mathrm{C}$ (hexane)

1ㅁN $\underline{\text { NMR: }} \quad\left(400 \mathrm{MHz}, \mathrm{CDCl}_{3}\right)$

7.30-7.26 (m, 6 H, 2 X m-, p-Ph), 7.13-7.10 (m, 2 H, o-Ph), 7.07-7.02 (m, 2 H, o$\mathrm{Ph}), 3.94$ (d, $J=8.8,1 \mathrm{H}, \mathrm{HC}(4)), 3.89$ (d, $J=9.0,1 \mathrm{H}, \mathrm{HC}(5)), 3.33-3.24$ (m, 4 $\left.\mathrm{H}, \mathrm{HC}\left(1^{\prime}\right)\right), 2.38$ (d, $\left.J=9.5,3 \mathrm{H}, \mathrm{NMe}\right), 2.37$ (d, $\left.J=9.2,3 \mathrm{H}, \mathrm{NMe}\right), 1.72-1.54$ $\left(\mathrm{m}, 6 \mathrm{H},\left(\mathrm{HC}\left(2^{\prime}, 3^{\prime}\right)\right)\right.$

${ }^{13} \mathrm{C} \mathrm{NMR}: \quad\left(100 \mathrm{MHz}, \mathrm{CDCl}_{3}\right)$

138.5 (d, $J=9.9$, ipso-Ph), 138.3 (d, $J=6.1$, ipso-Ph), $128.4(m-\mathrm{Ph}), 127.96$ (m$\mathrm{Ph}), 127.89$ (o-Ph), $127.6(o-\mathrm{Ph}), 72.4$ (d, $J=11.5, \mathrm{C}(4)), 70.5$ (d, $J=10.7, \mathrm{C}(5))$, 45.4 (d, $\left.J=3.1, \mathrm{C}\left(1^{\prime}\right)\right), 30.1$ (d, $\left.J=2.3, \mathrm{NMe}\right), 29.5$ (d, $\left.J=4.6, \mathrm{NMe}\right), 26.8$ (d, $J=$ $\left.3.8, \mathrm{C}\left(2^{\prime}\right)\right), 24.8\left(\mathrm{C}\left(3^{\prime}\right)\right)$ 
31

27.35

IR: $\quad\left(\mathrm{CHCl}_{3}\right)$

3067 (w, CH), 3036 (w, CH), 3020 (w, CH), 3012 (w, CH), 2979 (m, CH), 2959

(m, CH), 2939 (s, CH), 2853 (m, CH), 2818 (w, CH), 1497 (w), 1469 (w), 1456

(m), 1446 (w), 1376 (w), 1359 (w), 1339 (w), 1294 (w), 1280 (w), 1241 (s), 1221

(w), 1207 (m), 1180 (s), 1162 (s), 1125 (w), 1071 (m), 1039 (w), 1019 (m), 998

(m), $962(\mathrm{~s}), 833(\mathrm{w}), 810(\mathrm{w}), 775(\mathrm{w}), 768(\mathrm{w}), 700(\mathrm{~s})$

MS: $\quad(\mathrm{EI}, 70 \mathrm{eV})$

$369\left(\mathrm{M}^{+}, 26\right), 250$ (23), 166 (15), 165 (12), 120 (32), $119\left(\mathrm{C}_{8} \mathrm{H}_{9} \mathrm{~N}^{+}, 36\right), 118$

(87), $84\left(\mathrm{C}_{5} \mathrm{H}_{10} \mathrm{~N}^{+}, 100\right), 60$ (19), 43 (17), 42 (12)

TLC: $\quad R_{f} 0.42(\mathrm{EtOAc} / i-\mathrm{PrOH}, 9 / 1)$

Opt. Rot.: $\quad[\alpha]_{\mathrm{D}}^{22}+18.2\left(c=1.3, \mathrm{CHCl}_{3}\right)$

Analysis: Calcd for $\mathrm{C}_{21} \mathrm{H}_{28} \mathrm{~N}_{3} \mathrm{OP}(369.45)$

Calculated: $\quad$ C, 68.27\%; H, 7.64\%; N, 11.37\%; P, 8.38\%

Found: $\quad$ C, $68.00 \% ; \quad H, 7.68 \% ; \quad N, 11.31 \% ; \quad$ P, $8.23 \%$

\section{(+)-(4R,5R)-1,3-Dimethyl-4,5-bis-(4-trifluoromethyl-phenyl)-2-piperidino-1,3,2-}

diazaphospholidine 2-Oxide $(R, R)-7 b$.

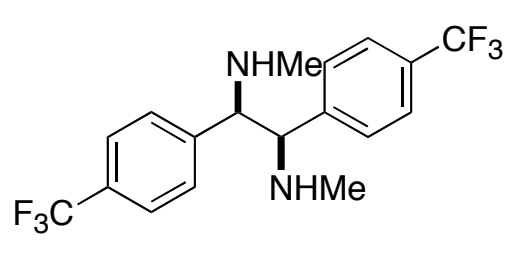

$(R, R)-19$

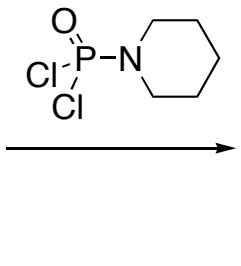

$\mathrm{F}_{3} \mathrm{C}$

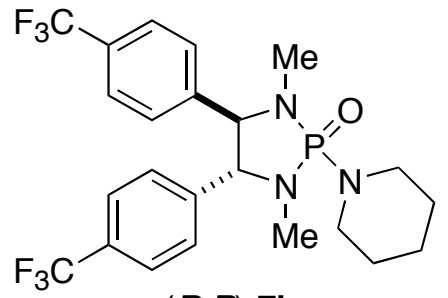

$(R, R)-7 \mathbf{b}$

Following Representative Procedure I from triethylamine $(230 \mu \mathrm{L}, 1.64 \mathrm{mmol}, 4.5$ equiv), $(R, R)-1^{2}(137 \mathrm{mg}, 0.364 \mathrm{mmol})$ and piperidinophosphoric dichloride (61 $\mu \mathrm{L}, 0.4 \mathrm{mmol}$, 1.1 equiv) in $70 \mathrm{~mL}$ of $\mathrm{CH}_{2} \mathrm{Cl}_{2}$ was obtained $75 \mathrm{mg}(41 \%)$ of $(R, R)-7 \mathbf{b}$ as white needles after column chromatography ( $\left.\mathrm{SiO}_{2}, \mathrm{TBME} / \mathrm{MeOH}, 19 / 1\right)$ and recrystallization (hexane). 
Analytical Data for $(+)-(R, R)-7 \mathbf{b}$ :

M.W.: $\quad 505.45$

mp: $\quad 138-139{ }^{\circ} \mathrm{C}$ (hexane)

1ㅁN NMR: $\quad\left(500 \mathrm{MHz}, \mathrm{CDCl}_{3}\right)$

$7.58\left(\mathrm{~d}, J=8.0,2 \mathrm{H}, \mathrm{HC}\left(3^{\prime}\right)\right), 7.57\left(\mathrm{~d}, J=7.8,2 \mathrm{H}, \mathrm{HC}\left(3^{\prime}\right)\right), 7.26(\mathrm{~d}, J=7.8,2 \mathrm{H}$, $\left.\mathrm{HC}\left(2^{\prime}\right)\right), 7.17$ (d, $\left.J=8.1,2 \mathrm{H}, \mathrm{HC}\left(2^{\prime}\right)\right), 3.98$ (d, $\left.J=8.7,1 \mathrm{H}, \mathrm{HC}(4)\right), 3.92$ (d, $J=$ 8.7, 1 H, HC(5)), 3.32-3.22 (m, 4 H, HC(1")), 3.38 (d, J = 10.4, 3 H, Me), 3.37 (d, $J=9.2,3 \mathrm{H}, \mathrm{Me}), 1.73-1.63$ (m, 2 H, HC(3")), 1.63-1.56 (m, 4 H, HC(2")).

\section{${ }^{13}$ C NMR: $\quad\left(125 \mathrm{MHz}, \mathrm{CDCl}_{3}\right)$}

$142.3\left(\mathrm{~d}, J=10.3\left(\mathrm{C}\left(1^{\prime}\right)\right), 142.2\left(\mathrm{~d}, J=6.6, \mathrm{C}\left(1^{\prime}\right)\right), 130.7\left(\mathrm{q}, J=32.2, \mathrm{C}\left(4^{\prime}\right)\right)\right.$, $128.2\left(\mathrm{C}\left(2^{\prime}\right)\right), 127.9\left(\mathrm{C}\left(2^{\prime}\right)\right), 125.7\left(\mathrm{br}, \mathrm{C}\left(3^{\prime}\right)\right), 123.98\left(\mathrm{q}, J=272.6, \mathrm{CF}_{3}\right), 123.90$ $\left(\mathrm{q}, J=271.7, \mathrm{CF}_{3}\right), 71.7(\mathrm{~d}, J=11.0, \mathrm{C}(4)), 70.0(\mathrm{~d}, J=11.1, \mathrm{C}(5)), 45.6(\mathrm{~d}, J$ =2.8, C(1")), 30.4 (d, $J=2.8, \mathrm{Me}), 29.6(\mathrm{~d}, J=4.6, \mathrm{Me}), 26.8\left(\mathrm{~d}, J=3.7, \mathrm{C}\left(2^{\prime \prime}\right)\right)$, $24.7\left(\mathrm{C}\left(3^{\prime \prime}\right)\right)$.

${ }^{19}$ F NMR: $\quad\left(470 \mathrm{MHz}, \mathrm{CDCl}_{3}\right)$

$-64.18,-64.22$.

31 $\underline{\text { P NMR: }} \quad\left(202 \mathrm{MHz}, \mathrm{CDCl}_{3}\right)$

29.54

IR: $\quad\left(\mathrm{CHCl}_{3}\right)$

$2981(w), 2940$ (w), 2880 (w), 2842 (w), 2820 (w), 1620 (w), 1467 (w), 1447 (w), 1419 (w), 1368 (w), 1325 (s), 1310 (w), 1300 (w), 1288 (w), 1244 (w), 1167 (m), 1132 (s), 1107 (m), 1068 (s), 1038 (w), 1017 (w), 1002 (w), 962 (m), 856 (w), $836(\mathrm{w})$.

$\underline{\mathrm{MS}}: \quad(\mathrm{CI})$

$534\left(\mathrm{M}^{+}+29,29\right), 507\left(\mathrm{M}^{+}+2,24\right), 506\left(\mathrm{M}^{+}+1,95\right), 505\left(\mathrm{M}^{+}, 17\right), 504\left(\mathrm{M}^{+}-1\right.$, 14), 487 (26), $486\left(\mathrm{M}^{+}-\mathrm{F}, 100\right), 84(35)$.

HRMS: (CI)

Calcd. for $\left(\mathrm{C}_{23} \mathrm{H}_{27} \mathrm{~F}_{6} \mathrm{~N}_{3} \mathrm{OP}\right)$

Found:
506.1796

506.1790 
Opt. Rot:: $[\alpha]_{\mathrm{D}}^{22}+0.6\left(\mathrm{CHCl}_{3}, \mathrm{c}=2.10\right)(+24.2$ at $405 \mathrm{~nm}$. $)$

TLC: $\quad R_{f} 0.38(\mathrm{TBME} / \mathrm{MeOH}, 20 / 1)$

HPLC: $\quad t_{R}((4 R, 5 R)-134 a) 20.73 \min ($ Daicel ChiralCel AD, hexane/EtOH, 97/3, 0.5 $\mathrm{mL} / \mathrm{min})$

Analysis: $\quad \mathrm{C}_{23} \mathrm{H}_{26} \mathrm{~F}_{6} \mathrm{~N}_{3} \mathrm{OP}$

Calcd: C, $54.66 \% ; \quad \mathrm{H}, \quad 5.18 \% ; \mathrm{N}, \quad 8.31 \%$.

Found: $\mathrm{C}, \quad 54.75 \% ; \quad \mathrm{H}, \quad 5.22 \% ; \mathrm{N}, \quad 7.84 \%$.

\section{(+)-(4R,5R)-1,3-Dimethyl-4,5-bis(4-methoxyphenyl)-2-piperidino-1,3,2-diazaphospholidine}

\section{2-Oxide $(R, R)-7 c$.}

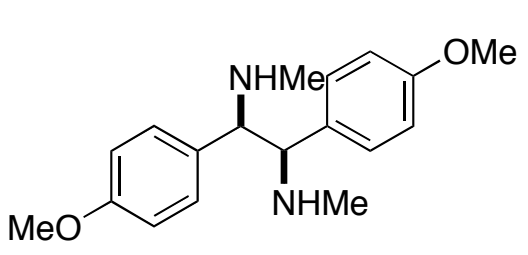

$(R, R)-20$
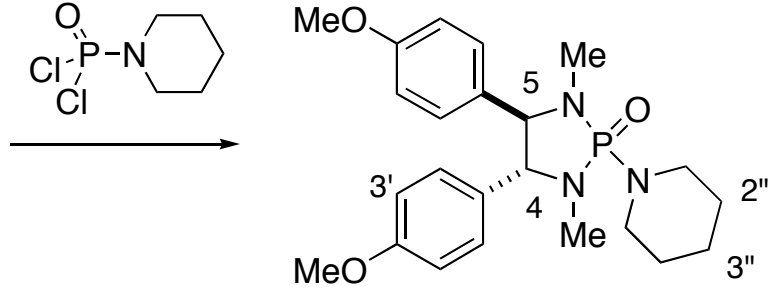

$(R, R)-7 \mathrm{c}$

To a solution of the diamine $(R, R)-\mathbf{2 0}^{2}(523 \mathrm{mg}, 1.74 \mathrm{mmol})$ and triethylamine $(610 \mu \mathrm{L}$, $4.35 \mathrm{mmol}, 2.5$ equiv) in $\mathrm{CH}_{2} \mathrm{Cl}_{2}(160 \mathrm{~mL})$ was added piperidinophosphoryl dichloride $(280 \mu \mathrm{L}$, $1.83 \mathrm{mmol}, 1.05$ equiv) at $0{ }^{\circ} \mathrm{C}$. The solution was then heated to reflux for $37 \mathrm{~h}$ and another portion of the dichloride (140 $\mu \mathrm{L}, 0.9 \mathrm{mmol}, 0.52$ equiv) was added. The reaction mixture was heated to reflux for $8 \mathrm{~h}$ further and then was poured into $5 \%$ aq. $\mathrm{HCl}(20 \mathrm{~mL})$. The phases were separated and the organic phase was washed with sat. aq. $\mathrm{NaHCO}_{3}$ solution $(20 \mathrm{~mL})$ and brine $(20 \mathrm{~mL})$, and then was dried ( $\left.\mathrm{Na}_{2} \mathrm{SO}_{4}\right)$ filtered and concentrated on a rotovap. The residue was purified by column chromatography (EtOAc/i-PrOH, 9/1) to afford $560 \mathrm{mg}(75 \%)$ of a white foam, which was recrystallized (hexane) to give $251 \mathrm{mg}(34 \%)$ of $(R, R)-7 \mathrm{c}$ as white crystals. Analytical Data for $(+)-(R, R)-7 \mathrm{c}$ :

M.W.: $\quad 429.50$

mp: $\quad 115-117^{\circ} \mathrm{C}$ (hexane) 
${ }^{1}$ H NMR: $\quad\left(400 \mathrm{MHz}, \mathrm{CDCl}_{3}\right)$

$7.03\left(\mathrm{~d}, J=8.1,2 \mathrm{H}, \mathrm{HC}\left(2^{\prime}\right)\right), 6.95\left(\mathrm{~d}, J=8.8,2 \mathrm{H}, \mathrm{HC}\left(2^{\prime}\right)\right), 6.80(\mathrm{~d}, J=8.8,2 \mathrm{H}$, $\left.\mathrm{HC}\left(3^{\prime}\right)\right), 6.79\left(\mathrm{~d}, J=8.8,2 \mathrm{H}, \mathrm{HC}\left(3^{\prime}\right)\right), 3.85(\mathrm{~d}, J=9.0,1 \mathrm{H}, \mathrm{HC}(4)), 3.79(\mathrm{~d}, 1 \mathrm{H}$, HC(5)), 3.78 (s, $3 \mathrm{H}, \mathrm{MeO}$ ), 3.78 (s, $3 \mathrm{H}, \mathrm{MeO}$ ), 3.26-3.20 (m, 4 H, HC(1")), 2.34 $(\mathrm{d}, J=10.2,3 \mathrm{H}, \mathrm{MeN}), 2.33(\mathrm{~d}, J=9.5,3 \mathrm{H}, \mathrm{MeN}), 1.68-1.54(\mathrm{~m}, 6 \mathrm{H}, \mathrm{HC}(2 "$, $3 "))$.

${ }^{13}$ C NMR: $\quad\left(100 \mathrm{MHz}, \mathrm{CDCl}_{3}\right)$

$159.23\left(\mathrm{C}\left(4^{\prime}\right)\right), 159.21\left(\mathrm{C}\left(4^{\prime}\right)\right), 130.4\left(\mathrm{~d}, J=9.9, \mathrm{C}\left(1^{\prime}\right)\right), 130.3\left(\mathrm{~d}, J=5.9, \mathrm{C}\left(1^{\prime}\right)\right)$, $129.0\left(\mathrm{C}\left(2^{\prime}\right)\right), 128.7\left(\mathrm{C}\left(2^{\prime}\right)\right), 113.72\left(\mathrm{C}\left(3^{\prime}\right)\right), 113.62\left(\mathrm{C}\left(3^{\prime}\right)\right), 72.1(\mathrm{~d}, J=10.7 \mathrm{C}(4))$, $70.2(\mathrm{~d}, J=10.7, \mathrm{C}(5)), 55.2(\mathrm{MeO}), 45.6(\mathrm{~d}, J=2.3, \mathrm{C}(1 ")), 30.0(\mathrm{~d}, J=3.1$, MeN), $29.4(\mathrm{~d}, J=4.6, \mathrm{MeN}), 26.8\left(\mathrm{~d}, J=3.1, \mathrm{C}\left(2^{\prime \prime}\right)\right), 24.8\left(\mathrm{C}\left(3^{\prime \prime}\right)\right)$.

31 $\underline{\text { P NMR: }} \quad\left(162 \mathrm{MHz}, \mathrm{CDCl}_{3}\right)$

27.11

IR: $\left(\mathrm{CHCl}_{3}\right)$

3020 (w), 2958 (m), 2938 (m), 2879 (w), 2851 (m), 2840 (m), 2817 (w), 1613 (m), 1587 (w), 1513 (s), 1464 (m), 1443 (m), 1303 (w), 1289 (w), 1246 (s), 1213 (w), 1173 (s), 1160 (s), 1070 (w), 1037 (m), 1022 (w), 997 (m), 961 (s), 849 (w), 839 (w), $833(\mathrm{w}), 796(\mathrm{~m}), 747$ (w), $773(\mathrm{w})$.

MS: $\quad(E I, 70 \mathrm{eV})$ $429\left(\mathrm{M}^{+}, 8\right), 280$ (5), 184 (10), 149 (26), 148 (12), 89 (28), 73 (100), 69 (12), 62 (46), 61 (12), 58 (46), 57 (12), 55 (17).

Opt. Rot.: $\quad[\alpha]_{\mathrm{D}}^{22}+49.9\left(\mathrm{CHCl}_{3}, \mathrm{c}=1.12\right)$

TLC: $\quad R_{f} 0.32(\mathrm{TBME} / \mathrm{MeOH}, 20 / 1)$

Analysis: $\quad \mathrm{C}_{2} 3 \mathrm{H}_{32} \mathrm{~N}_{3} \mathrm{O}_{3} \mathrm{P}$

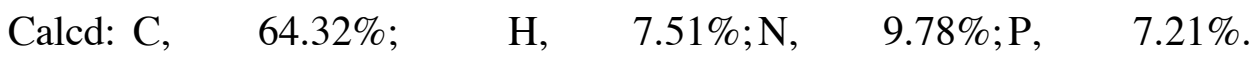

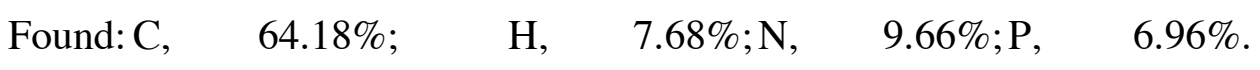


(+)-(4S,5S)-1,3-Dimethyl-4,5-bis(3,5-dimethylphenyl)-2-piperidino-1,3,2-diazaphospholidine

2-Oxide $(S, S)$-7d.

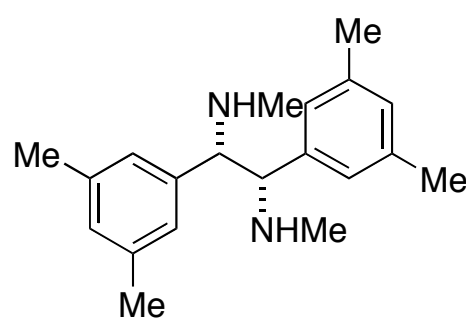

$(S, S)-21$
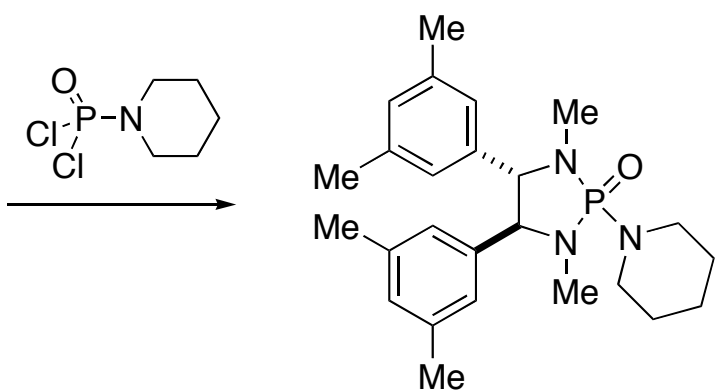

$(S, S)-7 \mathbf{d}$

Following Representative Procedure I from triethylamine (185 $\mu \mathrm{L}, 1.33$ mmol, 2.4 equiv), the diamine $(S, S)-\mathbf{2 1}{ }^{2}(164 \mathrm{mg}, 0.55 \mathrm{mmol})$ and piperidinophosphoryl dichloride (86 $\mu \mathrm{L}$, $0.56 \mathrm{mmol}, 1.02$ equiv) in $65 \mathrm{~mL}$ of $\mathrm{CH}_{2} \mathrm{Cl}_{2}$ was obtained $145 \mathrm{mg}(62 \%)$ of $(S, S)-7 \mathbf{d}$ as a white foam after column chromatography ( $\left.\mathrm{SiO}_{2}, \mathrm{TBME} / \mathrm{MeOH}, 19 / 1\right)$. An analytical sample was obtained by recrystallization from hexane.

Analytical Data for $(S, S)-7 \mathbf{d}$ :

M.W.: $\quad 425.56$

mp: $\quad 149-150{ }^{\circ} \mathrm{C}$

1

6.91 (s, 1 H, HC (4')), 6.89 (s, 1 H, HC(4')), 6.74 (br, 2 H, HC(2')), 6.64 (s, 2 H, $\left.\mathrm{H}\left(2^{\prime}\right)\right), 3.83$ (d, $\left.J=8.6,1 \mathrm{H}, \mathrm{HC}(4)\right), 3.78$ (d, $\left.J=8.6,1 \mathrm{H}, \mathrm{HC}(5)\right), 3.28-3.20$ (m, $4 \mathrm{H}, \mathrm{HC}(1 ")), 2.37$ (d, $J=10.2,3 \mathrm{H}, \mathrm{MeN}), 2.36$ (d, $J=9.1,3 \mathrm{H}, \mathrm{MeN}), 2.25$ (s, 3 H, Me-Ar), 2.24 (s, 3 H, Me-Ar), 1.70-1.56 (m, 6 H, HC(2", 3").

${ }^{13} \underline{\mathrm{C} \mathrm{NMR}}: \quad\left(125 \mathrm{MHz}, \mathrm{CDCl}_{3}\right)$

$138.9\left(\mathrm{~d}, J=10.1 \mathrm{C}\left(1^{\prime}\right)\right), 138.6\left(\mathrm{~d}, J=6.4, \mathrm{C}\left(1^{\prime}\right)\right), 137.7\left(\mathrm{C}\left(3^{\prime}\right)\right), 129.52\left(\mathrm{C}\left(4^{\prime}\right)\right)$, $129.47\left(\mathrm{C}\left(4^{\prime}\right)\right), 125.6\left(\mathrm{C}\left(2^{\prime}\right)\right), 125.4\left(\mathrm{C}\left(2^{\prime}\right)\right), 72.1$ (d, $\left.J=11.0, \mathrm{C}(4)\right), 70.3$ (d, $J=$ 11.0, C(5)), $45.6\left(\mathrm{~d}, J=2.8, \mathrm{C}\left(1^{\prime \prime}\right)\right), 30.2$ (d, $\left.J=3.7, \mathrm{MeN}\right), 29.6(\mathrm{~d}, J=4.6$, $\mathrm{MeN}), 26.8\left(\mathrm{~d}, J=3.7, \mathrm{C}\left(2^{\prime \prime}\right)\right), 24.8\left(\mathrm{C}\left(3^{\prime \prime}\right)\right), 21.3(\mathrm{Me}-\mathrm{Ar}), 21.2(\mathrm{Me}-\mathrm{Ar})$. 
31 P NMR: $\quad\left(162 \mathrm{MHz}, \mathrm{CDCl}_{3}\right)$

27.48

IR: $\quad\left(\mathrm{CHCl}_{3}\right)$

$2981(\mathrm{~m}), 2938$ (s), 2853 (m), $2816(\mathrm{w}), 1609$ (m), 1463 (m), 1446 (m), $1376(\mathrm{~m})$, 1359 (w), 1339 (m), 1305 (w), 1281 (w), 1258 (m), 1244 (w), 1156 (s), 1124 (w), $1071(\mathrm{~m}), 1033$ (w), 1022 (m), 998 (m), 961 (s), 923 (w), $857(\mathrm{w}), 822$ (w), 701 (w), $660(\mathrm{w}), 608(\mathrm{w})$.

MS: $\quad(E I, 70 \mathrm{eV})$

$426\left(\mathrm{M}^{+}+1,36\right), 425\left(\mathrm{M}^{+}, 97\right), 424\left(\mathrm{M}^{+}-1,13\right), 410$ (15), 344 (11), 340 (10), 320 (10), 279 (15), 278 (65), 263 (16), 194 (43), 193 (47), 148 (68), 147 (47), 146 (93), 132 (61), 119 (17), 84 (100), 60 (26).

Opt. Rot.: $\quad[\alpha]_{\mathrm{D}}^{22}+7.2\left(\mathrm{CHCl}_{3}, \mathrm{c}=1.00\right)$

TLC: $\quad R_{f} 0.47(\mathrm{TBME} / \mathrm{MeOH}, 20 / 1)$

HPLC: $\quad t_{R}((4 S, 5 S)-7 d) 9.93 \mathrm{~min}$ (Daicel ChiralCel AD, hexane/EtOH, 97/3, $0.5 \mathrm{~mL} / \mathrm{min}$ ) Analysis: $\quad \mathrm{C}_{2} 5 \mathrm{H} 36 \mathrm{~N}_{3} \mathrm{OP}$

Calcd: $\mathrm{C}, \quad 70.56 \% ; \quad \mathrm{H}, \quad 8.53 \% ; \mathrm{N}, \quad 9.87 \%$. Found: $\mathrm{C}, \quad 70.74 \% ; \quad \mathrm{H}, \quad 8.63 \% ; \mathrm{N}, \quad 9.82 \%$. $(S, S)-7$ e.

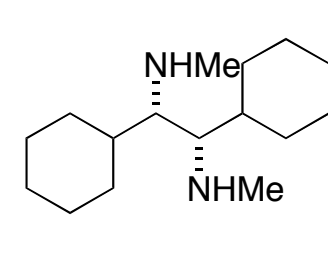

$(S, S)-22$
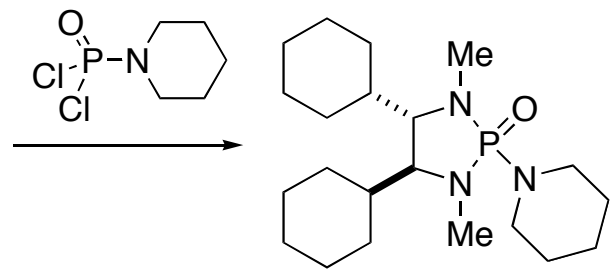

$(S, S)-7 e$

Following Representative Procedure I, to a solution of the diamine $(S, S)-\mathbf{2 2}^{2}$ (230 mg, $0.911 \mathrm{mmol})$ and triethylamine ( $305 \mu \mathrm{L}, 2.19 \mathrm{mmol}, 2.4$ equiv) in $\mathrm{CH}_{2} \mathrm{Cl}_{2}(100 \mathrm{~mL})$ was added piperidinophosphoryl dichloride $\left(153 \mu \mathrm{L}, 0.929 \mathrm{mmol}, 1.02\right.$ equiv) dropwise at $0{ }^{\circ} \mathrm{C}$. The 
solution was then heated to reflux for $66 \mathrm{~h}$ and then was poured into $5 \%$ aq. $\mathrm{HCl}$ solution. The phases were separated and the organic layer was washed with sat. aq. $\mathrm{NaHCO}_{3}$ solution and brine then was dried $\left(\mathrm{Na}_{2} \mathrm{SO}_{4}\right)$ and concentrated on a rotavap. The residue was purified by column chromatography $\left(\mathrm{SiO}_{2}, \mathrm{EtOAc} / \mathrm{i}-\mathrm{PrOH} 19 / 1\right)$ to give $172 \mathrm{mg}(49 \%)$ of $(S, S)-7 \mathrm{e}$ as a white foam.

Analytical Data for $(S, S)-7 \mathbf{e}$

M.W.: $\quad 381.55$

${ }^{1} \underline{\text { H NMR: }} \quad\left(500 \mathrm{MHz}, \mathrm{CDCl}_{3}\right)$

3.18-2.98 (m, 4 H, H(1")), 2.64 (d, $J=16.1,1 \mathrm{H}, \mathrm{HC}(4)), 2.52$ (d, $J=9.1,3 \mathrm{H}$, Me), $2.51(\mathrm{~d}, J=2.7,3 \mathrm{H}, \mathrm{Me}), 2.35(\mathrm{dd}, J=7.3,7.1,1 \mathrm{H}, \mathrm{HC}(5)), 1.97$ (d, $J=$ $12.8,1 \mathrm{H}), 1.86(\mathrm{~d}, J=12.3,1 \mathrm{H}), 1.80-0.90\left(\mathrm{~m}, 26 \mathrm{H}, \mathrm{CH}_{2}\right)$.

${ }^{13}$ C NMR: $\quad\left(125 \mathrm{MHz}, \mathrm{CDCl}_{3}\right)$

$66.9(\mathrm{~d}, J=15.7, \mathrm{C}(4)), 66.0(\mathrm{~d}, J=11.0, \mathrm{C}(5)), 45.4\left(\mathrm{~d}, J=2.8, \mathrm{C}\left(1^{\prime \prime}\right)\right), 45.0(\mathrm{~d}, J$ $\left.=2.8, \mathrm{C}\left(1^{\prime}\right)\right), 41.0\left(\mathrm{C}\left(1^{\prime}\right)\right), 33.7(\mathrm{~d}, J=5.5, \mathrm{Me}), 30.6(\mathrm{~d}, J=6.4, \mathrm{Me}), 30.1,29.6$, 29.1, 27.7, 26.8 (d, $\left.J=3.7, \mathrm{C}\left(2^{\prime \prime}\right)\right), 26.52,26.45\left(\mathrm{~d}, J=3.7, \mathrm{C}\left(2^{\prime \prime}\right)\right), 26.3,26.2$, $24.9\left(\mathrm{C}\left(3^{\prime \prime}\right)\right)$.

31 $\underline{\text { P NMR: }} \quad\left(202 \mathrm{MHz}, \mathrm{CDCl}_{3}\right)$

27.48

IR: $\quad\left(\mathrm{CHCl}_{3}\right)$

2931 (s), 2854 (s), 1450 (m), 1374 (w), 1358 (w), 1338 (w), 1301 (w), 1260 (w), 1249 (w), 1161 (s), 1124 (w), 1070 (m), 1027 (w), 1008 (w), 977 (w), 961 (m), $896(\mathrm{w})$.

MS: $\quad(\mathrm{CI})$

$410\left(\mathrm{M}^{+}+29,16\right), 383(\mathrm{M}+2,24), 382(\mathrm{M}+1,100), 381(\mathrm{M}+, 11), 380(\mathrm{M}-1,45)$, 299 (15), 298 (87).

HRMS: (CI)

Calcd. for $\left(\mathrm{C}_{21} \mathrm{H}_{41} \mathrm{~N}_{3} \mathrm{OP}\right) \quad 382.2987$

Found: 382.2991

Opt. Rot.: $\quad[\alpha]_{\mathrm{D}}^{22}+1.5\left(\mathrm{CHCl}_{3}, \mathrm{c}=2.31\right)$ 
TLC: $\quad R_{f} 0.52(\mathrm{EtOAc} / i-\mathrm{PrOH}, 19 / 1)$

\section{Representative Procedure II: Synthesis of Phosphoramides from Phosphorus Trichloride. ( \pm )-1,3-Dimethyl-4,5-bis(1-naphthyl)-2-piperidino-1,3,2-diazaphospholidine 2-Oxide ( \pm )-7f}

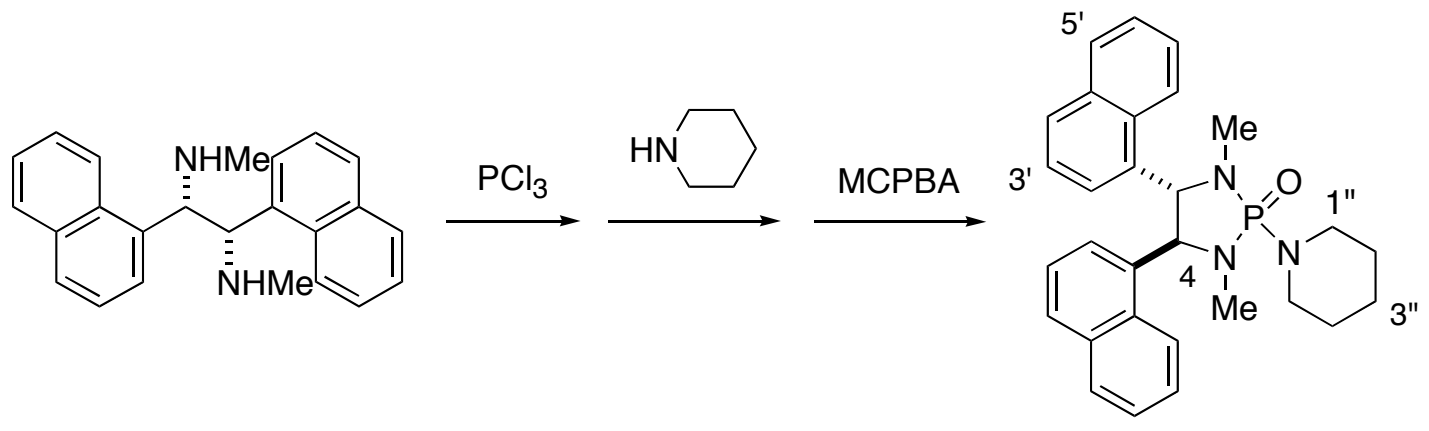

$( \pm)-23$

$( \pm)-7 f$

To a stirred cold $\left(-78{ }^{\circ} \mathrm{C}\right)$ solution of $\mathrm{PCl}_{3}(97 \mu \mathrm{L}, 1.11 \mathrm{mmol}, 1.02$ equiv) in $3 \mathrm{~mL}$ of $\mathrm{CH}_{2} \mathrm{Cl}_{2}$ was added ( \pm )- $N, N^{\prime}$-dimethyl-1,2-bis(1-naphthyl)-1,2-ethanediamine $( \pm)-\mathbf{2 3}^{2}$ (370 mg, $1.09 \mathrm{mmol})$ in $3 \mathrm{~mL}$ of $\mathrm{CH}_{2} \mathrm{Cl}_{2}$ via a syringe under nitrogen. Then triethylamine $(318 \mu \mathrm{L}, 2.28$ mmol, 2.1 equiv) was added at the same temperature. The mixture was warmed to room temperature, was stirred for $5 \mathrm{~h}$, and then was evaporated under reduced pressure to remove the excess reagents $\left(\mathrm{PCl}_{3}\right.$ and triethylamine).

The residue was suspended in $4 \mathrm{~mL}$ of $\mathrm{CH}_{2} \mathrm{Cl}_{2}$ and piperidine $(269 \mu \mathrm{L}, 2.72 \mathrm{mmol}, 2.5$ equiv) at room temperature and the mixture was stirred under nitrogen, for $18 \mathrm{~h}$ and then was concentrated under reduced pressure to remove excess piperidine.

The residue was dissolved in $5 \mathrm{~mL}$ of $\mathrm{CH}_{2} \mathrm{Cl}_{2}$ and a solution of MCPBA $(70 \%, 563 \mathrm{mg}$, $2.28 \mathrm{mmol}, 2.1$ equiv) in $5 \mathrm{~mL}$ of $\mathrm{CH}_{2} \mathrm{Cl}_{2}$ was added dropwise at $0^{\circ} \mathrm{C}$. The mixture was allowed to warm to room temperature and was stirred for $24 \mathrm{~h}$. Then, sat. aq. NaHCO3 solution (10 mL) was added with stirring. The aqueous layer was separated and was extracted with $\mathrm{CH}_{2} \mathrm{Cl}_{2}(2 \mathrm{X}$ $10 \mathrm{~mL})$. The combined organic solutions were washed with sat. aq. $\mathrm{NaHCO}_{3},(10 \mathrm{~mL})$ dried (MgSO4), filtered, and evaporated to give a residue, which was purified by column chromatography ( $\mathrm{SiO}_{2}, \mathrm{EtOAc} / \mathrm{i}-\mathrm{PrOH}, 19 / 1$ to 9/1) to afford $196 \mathrm{mg}(38 \%)$ of $( \pm)-7 f$. An analytical sample was obtained by recrystallization from hexane. 
Analytical Data for ( \pm -7f:

M.W.: $\quad 469.57$

mp: $\quad 183-186^{\circ} \mathrm{C}$ (hexane)

1ㅁN $\underline{\text { NMR: }} \quad\left(500 \mathrm{MHz}, \mathrm{CDCl}_{3}\right)$

mixture of rotamers: $9.33(\mathrm{~d}, J=8.6), 8.12(\mathrm{~d}, J=5.1), 7.90(\mathrm{~d}, J=8.1), 7.87(\mathrm{~d}$, $J=7.1$ ), 7.78-7.52 (m), 7.16 (dd, $J=7.7,7.5), 7.10-6.92$ (br), 6.83 (dd, $J=8.1$, 7.1), 6.74-6.58(br), 6.48 (d, $J=6.8$ ), 6.37 (d, $J=8.8$ ), 5.54 (d, $J=9.1$ ), 5.08-4.92 (br), 4.33 (d, $J=9.3$ ), 3.46-3.32 (br, HC(1")), 2.48 (d, $J=10.4), 2.44$ (d, $J=9.0$, Me), 2.43 (d, $J=10.5, \mathrm{Me}), 1.82-1.62\left(\mathrm{br}, \mathrm{HC}\left(2^{\prime \prime}, 3^{\prime \prime}\right)\right)$.

\section{${ }^{13} \underline{\mathrm{C} \mathrm{NMR}}: \quad\left(125 \mathrm{MHz}, \mathrm{CDCl}_{3}\right)$}

mixture of rotamers: 136.1, 135.0, 133.3, 132.4, 132.2, 131.7, 131.4, 129,6, 129.2, 128.9, 128.1, 127.2, 126.0, 125.8, 125.4, 125.3, 125.1, 124.7, 124.4, 124.2, 123.2, 122.1, 121.5, 61.2, 46-45 (br, C(1")), 31-29 (br, Me), 26.89 (d, J = 2.8, $\left.\mathrm{C}\left(2^{\prime \prime}\right)\right), 24.85\left(\mathrm{C}\left(3^{\prime \prime}\right)\right)$.

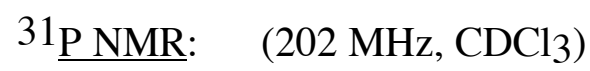

28.20 (br), $26.21,25.63$ (minor).

IR: $\quad\left(\mathrm{CHCl}_{3}\right)$

3059 (w), 3018 (w), 2981 (m), 2970 (m), 2958 (m), 2939 (m), 2883 (w), 2853

(m), 2822 (w), 1597 (w), 1512 (w), 1466 (w), 1446 (w), 1396 (w), $1376(\mathrm{w}), 1362$

(w), 1352 (w), 1339 (m), 1326 (w), 1289 (m), 1257 (w), 1243 (m), 1165 (s), 1150

(m), 1124 (w), 1071 (w), 1048 (w), 1020 (m), 981 (m), 962 (s), 865 (w), 852 (w), $834(\mathrm{w}), 815(\mathrm{w}), 804(\mathrm{w}), 661(\mathrm{w}), 647(\mathrm{w})$.

MS: $\quad(\mathrm{FAB})$

$471\left(\mathrm{M}^{+}+2,34\right), 470\left(\mathrm{M}^{+}+1,100\right), 469\left(\mathrm{M}^{+}, 11\right), 468\left(\mathrm{M}^{+}-1,13\right), 216(18), 170$ (10), 168 (14).

TLC: $\quad R_{f} 0.40(\mathrm{TBME} / \mathrm{MeOH}, 20 / 1)$

Analysis: $\quad \mathrm{C}_{2} 9 \mathrm{H}_{32} \mathrm{~N}_{3} \mathrm{OP}$

Calcd: C, 74.18\%; $\mathrm{H}, \quad 6.87 \% ; \mathrm{N}, \quad 8.95 \%$.

Found: C, 74.10\%; H, $6.75 \% ; \mathrm{N}, \quad 8.88 \%$. 
(+)-(4S,5S)-1,3-Dimethyl-4,5-bis-(2-naphthyl)-2-piperidino-1,3,2-diazaphospholidine 2-

\section{Oxide $(S, S)-7 \mathrm{~g}$.}

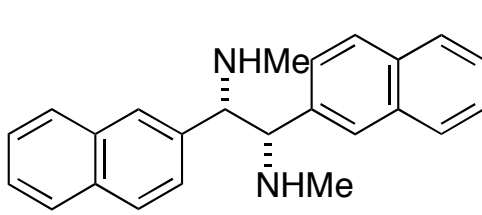

$(S, S)-24$
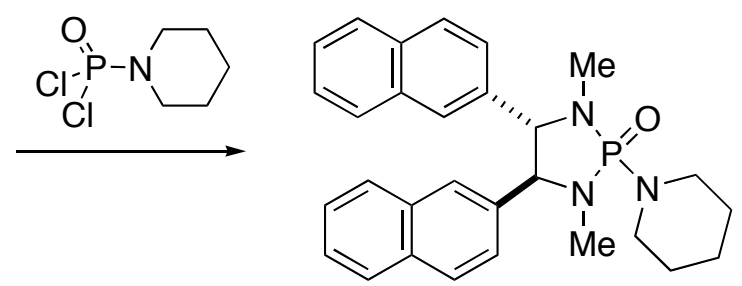

$(S, S)-\mathbf{7 g}$

Following Representative Procedure I from triethylamine $(185 \mu \mathrm{L}, 1.32 \mathrm{mmol}, 3.5$ equiv), the diamine $(S, S)-\mathbf{2 4}^{2}(180 \mathrm{mg}, 0.529 \mathrm{mmol})$ and piperidinophosphoryl dichloride $(89 \mu \mathrm{L}$, $0.582 \mathrm{mmol}, 1.1$ equiv) in $50 \mathrm{~mL}$ of $\mathrm{CH}_{2} \mathrm{Cl}_{2}$ was obtained $75 \mathrm{mg}(30 \%)$ of $(S, S)-7 \mathrm{~g}$ as white crystals after column chromatography ( $\left.\mathrm{SiO}_{2}, \mathrm{EtOAc} / i-\mathrm{PrOH}, 9 / 1\right)$ and recrystallization (hexane). Analytical Data for $(S, S)-7 \mathrm{~g}$ :

M.W.: 469.59

mp: $\quad 205-206^{\circ} \mathrm{C}$ (hexane)

1슬 $: \quad\left(500 \mathrm{MHz}, \mathrm{CDCl}_{3}\right)$

7.86-7.78 (m, $4 \mathrm{H}, \mathrm{Ar}), 7.69$ (d, J= 7.7, $1 \mathrm{H}, \mathrm{Ar}), 7.66$ (d, J= 7.9, $1 \mathrm{H}, \mathrm{Ar}), 7.54$ (br, $\left.1 \mathrm{H}, \mathrm{HC}\left(1^{\prime}\right)\right), 7.50-7.40$ (m, $\left.5 \mathrm{H}, \mathrm{Ar}\right), 7.37$ (br, $\left.1 \mathrm{H}, \mathrm{HC}\left(1^{\prime}\right)\right), 7.32$ (dd, $J=8.5$, 1.5, $\left.1 \mathrm{H}, \mathrm{HC}\left(3^{\prime}\right)\right), 4.24(\mathrm{~d}, J=8.6,1 \mathrm{H}, \mathrm{HC}(4)), 4.19$ (d, $\left.J=8.6,1 \mathrm{H}, \mathrm{HC}(5)\right)$, 3.40-3.38 (m, $\left.4 \mathrm{H}, \mathrm{HC}\left(1^{\prime \prime}\right)\right), 2.43$ (d, $J=10.4$, Me), 2.42 (d, $\left.J=9.13 \mathrm{H}, \mathrm{Me}\right)$, 1.72-1.60 (m, $\left.6 \mathrm{H}, \mathrm{HC}\left(2^{\prime \prime}, 3^{\prime \prime}\right)\right)$.

${ }^{13} \underline{\mathrm{C} \mathrm{NMR}}: \quad\left(125 \mathrm{MHz}, \mathrm{CDCl}_{3}\right)$

$136.1\left(\mathrm{~d}, J=10.2, \mathrm{C}\left(2^{\prime}\right)\right), 136.0\left(\mathrm{~d}, J=7.5, \mathrm{C}\left(2^{\prime}\right)\right), 133.29\left(\mathrm{C}\left(9^{\prime}\right)\right), 133.27\left(\mathrm{C}\left(9^{\prime}\right)\right)$, $133.14\left(\mathrm{C}\left(10^{\prime}\right)\right), 133.11$ (C(10')), 128.5 (Ar), 128.4 (Ar), 127.9 (Ar), 127.9 (Ar), 127.7 (Ar), 127.4 (Ar), 127.2 (Ar), 126.2 (Ar), 126.08 (Ar), 126.02 (Ar), 125.5 (Ar), 124.7 (Ar), 72.2 (d, $J=12.0, \mathrm{C}(4)), 70.6(\mathrm{~d}, J=11.0, \mathrm{C}(5)), 45.7\left(\mathrm{C}\left(1^{\prime \prime}\right)\right)$, $30.3(\mathrm{Me}), 29.6(\mathrm{~d}, J=3.7, \mathrm{Me}), 26.8\left(\mathrm{~d}, J=3.7, \mathrm{C}\left(2^{\prime \prime}\right)\right), 24.8\left(\mathrm{C}\left(3^{\prime \prime}\right)\right)$. 
31 P NMR: $\quad\left(202 \mathrm{MHz}, \mathrm{CDCl}_{3}\right)$

27.38

IR: $\left(\mathrm{CHCl}_{3}\right)$

3059 (w), 2982 (m), 2973 (m), 2963 (m), 2939 (m), 2904 (w), 2882 (w), 2852

(m), 2822 (w), 1602 (w), 1509 (w), 1467 (w), 1445 (w), 1375 (m, 1361 (w), 1339

(m), 1326 (w), 1319 (w), 1289 (m), 1269 (w), 1244 (m), 1162 (w), 1150 (m), 1123 (m), 1071 (m), 1026 (m), 1019 (m), 1005 (m), 961 (s), 953 (m), 896 (w), $883(w), 962(w), 832(w), 660(w)$.

MS: $\quad(E I, 70 \mathrm{eV})$

$470\left(\mathrm{M}^{+}+1\right), 469\left(\mathrm{M}^{+}, 70\right), 301$ (10), 300 (42), 216 (21), 215 (17), 170 (33), 169

(81), 168 (100), 153 (14), 141 (23), 128 (11), 60 (18).

Opt. Rot.: $\quad[\alpha]_{\mathrm{D}}^{22}+80.7\left(\mathrm{CHCl}_{3}, \mathrm{c}=1.38\right)$

TLC: $\quad R_{f} 0.39$ (TBME / MeOH, 20 / 1)

Analysis: $\quad \mathrm{C}_{2} 2 \mathrm{H}_{32} \mathrm{~N}_{3} \mathrm{OP}$

Calcd: $\mathrm{C}, \quad 74.18 \% ; \quad \mathrm{H}, \quad 6.87 \% ; \mathrm{N}, \quad 8.95 \% ; \mathrm{P}, \quad 6.60 \%$.

Found: $\mathrm{C}, \quad 74.12 \% ; \quad \mathrm{H}, \quad 6.75 \% ; \mathrm{N}, \quad 8.68 \% ; \mathrm{P}, \quad 6.72 \%$.

(4S,5S)-4,5-Diphenyl-2-(1-piperidinyl)-1,3,2-diazaphospholidine 2-Oxide (S,S)-7h.

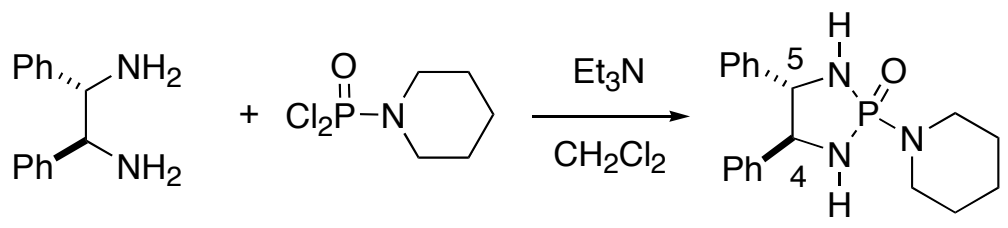

$(S, S)-7 \mathbf{h}$

Following Representative Procedure I, from triethylamine $(0.63 \mathrm{~mL}, 4.5 \mathrm{mmol}, 3.0$ equiv), (S,S)-1,2-diphenyl-1,2-ethanediamine ${ }^{1}(0.318 \mathrm{~g}, 1.5 \mathrm{mmol})$ and 1-piperidinylphosphoric dichloride $\left(0.23 \mathrm{~mL}, 1.5 \mathrm{mmol}, 1.0\right.$ equiv) in $\mathrm{CH}_{2} \mathrm{Cl}_{2}(14+8+8 \mathrm{~mL})$ was obtained $0.301 \mathrm{~g}(59 \%)$ of $(S, S)-7 \mathbf{h}$ as white crystals after column chromatography $\left(\mathrm{SiO}_{2}, \mathrm{EtOAc} / \mathrm{i}-\mathrm{PrOH} 10 / 1\right)$ and recrystallization $\left(\mathrm{CHCl}_{3} /\right.$ hexane). Anal. Calcd for $\mathrm{C}_{19} \mathrm{H}_{24} \mathrm{~N}_{3} \mathrm{OP}(341,37)$ : C, 66.85; H, 7.09; $\mathrm{N}, 12.31$. Found: C, 66.52; H, 6.90; N, 12.13 . 
Representative Procedure III: Synthesis of Phosphoramides from Aminophosphorus Dichloride. (4S,5S)-(+)-1,3-Diethyl-4,5-diphenyl-2-piperidino-1,3,2-diazaphospholidine 2Oxide $(S, S)-7 i)$

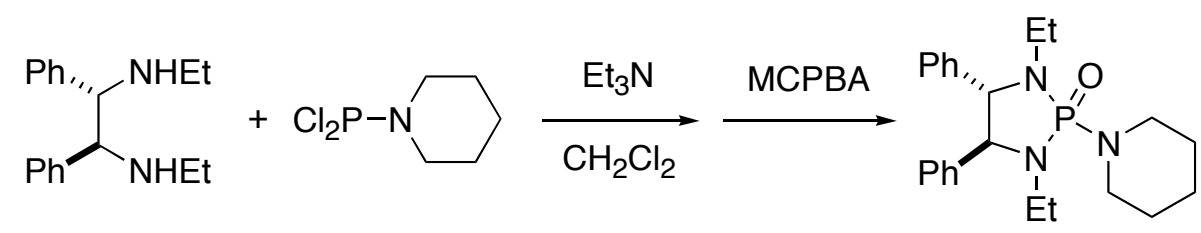

$(S, S)-25$

$(S, S)-7 \mathbf{i}$

To a solution of triethylamine $\left(0.66 \mathrm{~mL}, 4.73 \mathrm{mmol}, 2.2\right.$ equiv) in $80 \mathrm{~mL}$ of $\mathrm{CH}_{2} \mathrm{Cl}_{2}$ at reflux was added a solution of $N, N^{\prime}$-diethyl-1,2-diphenyl-1,2-ethanediamine $(S, S)$-25 ${ }^{3}$ (578 $\mathrm{mg}$, $2.15 \mathrm{mmol})$ in $5 \mathrm{~mL}$ of $\mathrm{CH}_{2} \mathrm{Cl}_{2}$ and a solution of piperidinophosphorus dichloride $(0.33 \mathrm{~mL}, 2.15$ mmol, 1.0 equiv) in $5 \mathrm{~mL}$ of $\mathrm{CH}_{2} \mathrm{Cl}_{2}$ simultaneously via a syringe pump over $2 \mathrm{~h}$. After addition the solution was heated to reflux for an additional $30 \mathrm{~min}$ and then was cooled back to $0^{\circ} \mathrm{C}$. A solution of MCPBA (85\%, $480 \mathrm{mg}, 2.37 \mathrm{mmol}, 1.1$ equiv) in $\mathrm{CH}_{2} \mathrm{Cl}_{2}(10 \mathrm{~mL})$ was then added via syringe and the solution was stirred at rt overnight. The slightly yellow solution was poured into a separatory funnel and washed with water $(20 \mathrm{~mL})$, sat. aq. $\mathrm{NaHCO}_{3}$ solution $(2 \mathrm{X}$ $20 \mathrm{~mL})$ and brine $(20 \mathrm{~mL})$. The organic layer was dried $\left(\mathrm{Na}_{2} \mathrm{SO}_{4}\right)$, and concentrated in vacuo to afford a crude product that was purified by column chromatography $\left(\mathrm{SiO}_{2}, \mathrm{CH}_{2} \mathrm{Cl}_{2} / \mathrm{MeOH}\right.$, 19/1) to give $549 \mathrm{mg}(64 \%)$ of $(S, S)-7 \mathbf{i}$ as a white solid. Recrystallization from hexane afforded $255 \mathrm{mg}(30 \%)$ analytically pure sample as white prisms.

Analytical Data for $(S, S)-7 \mathbf{i}$ :

mp: $\quad 150-152{ }^{\circ} \mathrm{C}$ (hexane)

1슬 $: \quad\left(500 \mathrm{MHz}, \mathrm{CDCl}_{3}\right)$

7.32-7.20 (m, $6 \mathrm{H}, \mathrm{Ph}), 7.20-7.12$ (m, $2 \mathrm{H}, \mathrm{Ph}), 7.06-7.00$ (m, $2 \mathrm{H}, \mathrm{Ph}), 4.12$ (d, J $=8.5,1 \mathrm{H}, \mathrm{HC}(4)), 4.04(\mathrm{~d}, J=8.5,1 \mathrm{H}, \mathrm{HC}(5)), 3.36-3.20\left(\mathrm{~m}, 4 \mathrm{H}, \mathrm{HC}\left(1^{\prime}\right)\right)$, 2.88-2.70 (m, $\left.4 \mathrm{H}, \mathrm{H}_{2} \mathrm{C}(\mathrm{Et})\right), 1.68-1.58$ (m, $\left.6 \mathrm{H}, \mathrm{HC}\left(2^{\prime}, 3^{\prime}\right)\right), 1.07$ (t, $J=7.2,3 \mathrm{H}$, $\mathrm{Me}), 0.97(\mathrm{t}, J=7.1, \mathrm{Me})$.

${ }^{13}$ C NMR: $\quad\left(125 \mathrm{MHz}, \mathrm{CDCl}_{3}\right)$

$139.2\left(\mathrm{~d}, J=10.1, \mathrm{C}\left(1^{\prime \prime}\right)\right), 138.9\left(\mathrm{~d}, J=6.4, \mathrm{C}\left(1^{\prime \prime}\right)\right), 128.4\left(\mathrm{C}\left(3^{\prime \prime}\right)\right), 128.3\left(\mathrm{C}\left(3^{\prime \prime}\right)\right)$, 
$128.1\left(\mathrm{C}\left(2^{\prime \prime}\right)\right), 127.90\left(\mathrm{C}\left(2^{\prime \prime}\right)\right), 127.87\left(\mathrm{C}\left(4^{\prime \prime}\right)\right), 127.6\left(\mathrm{C}\left(4^{\prime \prime}\right)\right), 69.5$ (d, $J=12.0$, C(4)), 68.1 (d, $J=11.0, \mathrm{C}(5)), 45.9\left(\mathrm{~d}, J=2.8, \mathrm{C}\left(1^{\prime}\right)\right), 38.0(\mathrm{~d}, J=2.8, \mathrm{C}(\mathrm{Et}))$, $37.1(\mathrm{~d}, J=4.6, \mathrm{C}(\mathrm{Et})), 26.6\left(\mathrm{~d}, J=4.6, \mathrm{C}\left(2^{\prime}\right)\right), 24,8\left(\mathrm{C}\left(3^{\prime}\right)\right), 13.6(\mathrm{Me}), 13.4$ (Me).

31 $\underline{\text { P NMR: }} \quad\left(202 \mathrm{MHz}, \mathrm{CDCl}_{3}\right)$

28.12

IR: $\quad\left(\mathrm{CHCl}_{3}\right)$

2973 (m), 2938 (s), 2902 (w), 2872 (m), 2853 (m), 1602 (w), 1492 (w), 1463 (w), 1455 (m), 1444 (m), 1377 (m), 1357 (m), 1338 (m), 1310 (w), 1278 (w), 1258 (w), 1161 (s), 1123 (m), 1071 (s), 1054 (m), 1022 (m), 961 (s), 917 (w), 912 (w), $901(\mathrm{w}), 879(\mathrm{w}), 854(\mathrm{w}), 838(\mathrm{w}), 700(\mathrm{w}), 659(\mathrm{w}), 613(\mathrm{w})$.

MS: $\quad(\mathrm{EI}, 70 \mathrm{eV})$

$397\left(\mathrm{M}^{+}, 34\right), 264$ (21), 235 (13), 180 (14), 179 (11), 134 (39), 133 (40), 132 (53), 118 (25), 104 (20), 91 (13), 84 (100), 74 (13), 55 (11).

Opt. Rot.: $[\alpha]_{\mathrm{D}}^{22}+6.2\left(\mathrm{CHCl}_{3}, \mathrm{c}=1.20\right)$

TLC: $\quad R_{f} 0.33\left(\mathrm{CH}_{2} \mathrm{Cl}_{2} / \mathrm{MeOH}, 19 / 1\right)$

Analysis: $\quad$ Calcd for $\mathrm{C}_{23} 3 \mathrm{H}_{32} \mathrm{~N}_{3} \mathrm{OP}$ (397.50)

Calcd: $\quad$ C $\quad 69.50 \% ; \quad \mathrm{H}, 8.11 \% ; \mathrm{N}, 10.57 \% ; \mathrm{P}, \quad 7.79 \%$.

Found: $\quad$ C, $69.48 \% ; \quad \mathrm{H}, 8.08 \% ; \mathrm{N}, 10.55 \% ; \mathrm{P}, 8.04 \%$. 
$(S, S)-1,3,4,5-T e t r a p h e n y l-2-p i p e r i d i n o-1,3,2-d i a z a p h o s p h o l i d i n e ~ 2-O x i d e ~(S, S)-7 j$.

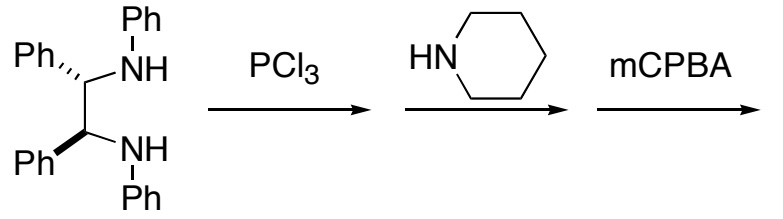

$(S, S)-26$

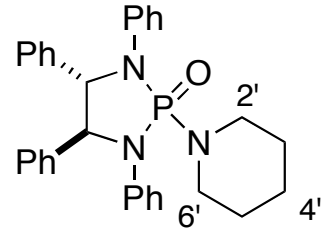

$(S, S)-7 \mathbf{j}$

Following Representative Procedure II, to a stirred cold $\left(-78{ }^{\circ} \mathrm{C}\right)$ solution of $\mathrm{PCl} 3$ (69 $\mathrm{mL}, 0.79 \mathrm{mmol}, 1.04$ equiv) in $3 \mathrm{~mL}$ of $\mathrm{CH}_{2} \mathrm{Cl}_{2}$ was added $(S, S)-N, N^{\prime}, 1,2$-tetraphenyl-1,2ethanediamine $(S, S)-\mathbf{2 6}^{4}$ (261 mg, $0.716 \mathrm{mmol}, 1.0$ equiv) in $3 \mathrm{~mL}$ of $\mathrm{CH}_{2} \mathrm{Cl}_{2}$ via a syringe under nitrogen. Then triethylamine $(0.22 \mathrm{~mL}, 1.57 \mathrm{mmol}, 2.2$ equiv) was added at the same temperature. The mixture was warmed to room temperature, was stirred for $5 \mathrm{~h}$, and then was evaporated under reduced pressure to remove the excess reagents ( $\mathrm{PCl}_{3}$ and triethylamine).

The residue was suspended in $4 \mathrm{~mL}$ of $\mathrm{CH}_{2} \mathrm{Cl}_{2}$ and piperidine $(0.21 \mathrm{~mL}, 2.15 \mathrm{mmol}, 3.0$ equiv) at room temperature and the mixture was stirred under nitrogen, for $18 \mathrm{~h}$ and then was concentrated under reduced pressure to remove excess piperidine.

The residue was dissolved in $5 \mathrm{~mL}$ of $\mathrm{CH}_{2} \mathrm{Cl}_{2}$ and a solution of MCPBA $(50 \%, 250 \mathrm{mg}$; $0.72 \mathrm{mmol}, 1.0 \mathrm{mmol}$ ) in $5 \mathrm{~mL}$ of $\mathrm{CH}_{2} \mathrm{Cl}_{2}$ was added dropwise at $0^{\circ} \mathrm{C}$. The mixture was allowed to warm to room temperature and was stirred for $24 \mathrm{~h}$. Then, sat. aq. $\mathrm{NaHCO}_{3}$ solution $(10 \mathrm{~mL})$ was added with stirring. The aqueous layer was separated and was extracted with $\mathrm{CH}_{2} \mathrm{Cl}_{2}(2 \times 10 \mathrm{~mL})$. The combined organic solutions were washed with sat. aq. $\mathrm{NaHCO}_{3},(10$ $\mathrm{mL})$ dried $\left(\mathrm{MgSO}_{4}\right)$, filtered, and evaporated to give a residue, which was purified by column chromatography ( $\mathrm{SiO}_{2}, \mathrm{CH}_{2} \mathrm{Cl}_{2}$ then $\mathrm{CH}_{2} \mathrm{Cl}_{2} / i-\mathrm{PrOH}, 100 / 1$ as eluents). Recrystallization from hexane afforded $196 \mathrm{mg}(55 \%)$ of $(S, S)-7 \mathbf{j}$ as a colorless amorphous solid.

Analytical Data for $(-)-(S, S)-7 \mathbf{j}:$

M.W.: 493.59

mp: $\quad 195-197^{\circ} \mathrm{C}$ (hexane)

${ }^{1} \underline{\text { H NMR: }} \quad\left(500 \mathrm{MHz}, \mathrm{CDCl}_{3}\right)$

7.25-7.20 (m, 8H), 7.19-7.13 (m, 6H), 7.103-7.06 (m, 2H), 7.00 (dd, J = 7.7, 0.9, 
2H), $6.94(\mathrm{t}, J=7.3,1 \mathrm{H}), 6.91(\mathrm{t}, J=6.4,1 \mathrm{H}), 4.93(\mathrm{~d}, J=8.1,1 \mathrm{H}, \mathrm{HC}(4 / 5))$, $4.88(\mathrm{dd}, J=8.3,6.8,1 \mathrm{H}, \mathrm{H}(5 / 4))$, 3.40-3.32 (m, 2H, HC(1')), 3.18-3.08 (m, 2H, $\left.\mathrm{HC}\left(1^{\prime}\right)\right), 1.46-1.38\left(\mathrm{~m}, 4 \mathrm{H}, \mathrm{HC}\left(2^{\prime}\right) \mathrm{H}_{2}\right), 1.18-1.06$ (m, 2H, HC(3'))

\section{${ }^{13} \underline{\mathrm{C} \mathrm{NMR}}: \quad\left(125 \mathrm{MHz}, \mathrm{CDCl}_{3}\right)$}

140.30 (d, $\left.J=3.7, \mathrm{C}\left(1^{\prime \prime}\right)\right), 140.29$ (d, $\left.J=6.6,(\mathrm{C} 1 ")\right), 138.38$ (d, $J=8.3, \mathrm{C}\left(1^{\prime \prime \prime}\right)$ ), $138.30\left(\mathrm{~d}, J=4.6,\left(\mathrm{C} 1{ }^{\prime \prime}\right)\right), 129.0,128.6,128.52,128.1,127.7,127.5,123.2,122.2$, $121.9,121.8,119.6,119.5,67.7(\mathrm{~d}, J=10.1, \mathrm{C}(4 / 5)), 66.7(\mathrm{~d}, J=12.0, \mathrm{C}(5 / 4))$, $45.4\left(\mathrm{~d}, J=2.8, \mathrm{C}\left(1^{\prime}\right)\right), 25.4\left(\mathrm{~d}, J=4.6, \mathrm{C}\left(2^{\prime}\right)\right), 24.4\left(\mathrm{C}\left(3^{\prime}\right)\right)$

31P NMR: $\quad\left(162 \mathrm{MHz}, \mathrm{CDCl}_{3}\right)$

17.30

IR: $\quad\left(\mathrm{CHCl}_{3}\right)$

2989(w), 2940(m), 2899(w), 2854(w), 1599(m), 1493(s), 1456(m), 1385(m), 1377(m), 1361(w), 1353(w), 1341(w), 1308(w), 1294(m), 1279(w), 1262(m), 124(w), 1165(w), 1120(m), 1069(m), 1029 (w), 1001(w), 967(s), 917 (w), 836(w), 699(w)

MS: $\quad(\mathrm{EI}, 70 \mathrm{eV})$

$494\left(\mathrm{M}^{+}+1,21\right), 493\left(\mathrm{M}^{+}, 50\right), 182$ (43), 181 (100), 180 (39), 104 (10), 84 (25), 77 (18)

Opt. Rot.: $\quad[\alpha]_{\mathrm{D}}^{22}-194\left(\mathrm{CHCl}_{3}, c=1.01\right)$

TLC: $\quad R_{f} 0.49(\mathrm{EtOAc})$

Analysis: $\quad \mathrm{C}_{3} 1 \mathrm{H} 32 \mathrm{~N}_{3} \mathrm{OP}$

Calcd: $\mathrm{C}, \quad 75.44 \% ; \quad \mathrm{H}, \quad 6.53 \% ; \mathrm{N}, \quad 8.51 \% ; \mathrm{P}, \quad 6.28 \%$.

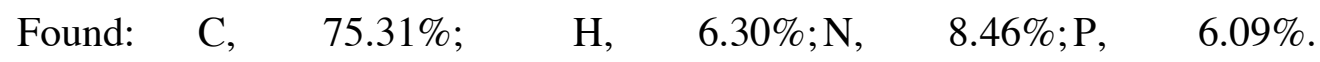




\section{$(R, R)-1,3,4,5$-Tetraphenyl-2-piperidino-1,3,2-diazaphospholidine 2-Oxide $(R, R)$-7j.}

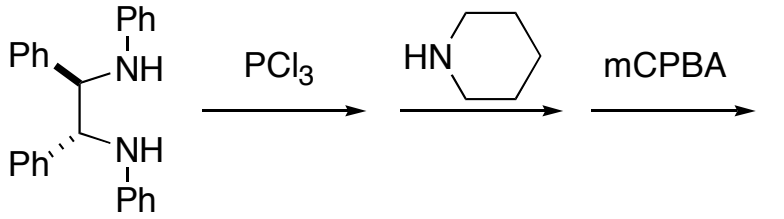

$(R, R)-26$

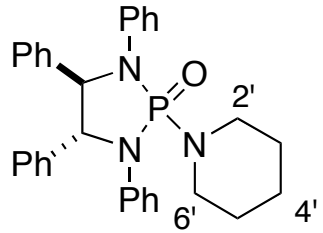

$(R, R)-\mathbf{7 j}$

Following Representative Procedure II, to a stirred cold $\left(-78^{\circ} \mathrm{C}\right)$ solution of $\mathrm{PCl}_{3}$ (69 $\mathrm{mL}, 0.79 \mathrm{mmol})$ in $3 \mathrm{~mL}$ of dry $\mathrm{CH}_{2} \mathrm{Cl}_{2}$ was added $(R, R)-\mathbf{2 6}{ }^{4}(261 \mathrm{mg}, 0.716 \mathrm{mmol})$ in $3 \mathrm{~mL}$ of dry $\mathrm{CH}_{2} \mathrm{Cl}_{2}$ via a syringe under nitrogen. Then triethylamine $(0.22 \mathrm{~mL}, 1.57 \mathrm{mmol})$ was added at the same temperature. The mixture was warmed to room temperature, stirred for $5 \mathrm{~h}$, and then condensed under reduced pressure to remove the excess reagents ( $\mathrm{PCl}_{3}$ and triethylamine).

The residue was suspended in $4 \mathrm{~mL}$ of dry $\mathrm{CH}_{2} \mathrm{Cl}_{2}$ and stirred, to which was added piperidine $(0.21 \mathrm{~mL}, 2.15 \mathrm{mmol})$ at room temperature under nitrogen. The mixture was stirred for $18 \mathrm{~h}$ and condensed under reduced pressure to remove excess piperidine.

The residue was dissolved in $5 \mathrm{~mL}$ of $\mathrm{CH}_{2} \mathrm{Cl}_{2}$. $m$-Chloroperbenzoic acid $(50 \%, 250 \mathrm{mg}$; $0.72 \mathrm{mmol}$ ) in $5 \mathrm{~mL}$ of $\mathrm{CH}_{2} \mathrm{Cl}_{2}$ was added dropwise at $0{ }^{\circ} \mathrm{C}$. The mixture was allowed to warm to room temperature and stirred for $24 \mathrm{~h}$. Then saturated aqueous $\mathrm{NaHCO}_{3}$ was added with stirring. The aqueous layer was separated and extracted with $\mathrm{CH}_{2} \mathrm{Cl}_{2}$ twice. The combined organic solutions were washed with saturated aqueous $\mathrm{NaHCO}_{3}$, dried over $\mathrm{MgSO} 4$, filtered, and evaporated to give a crude material, which was chromatographed on silica gel $\left(\mathrm{CH}_{2} \mathrm{Cl}_{2}\right.$ then $\mathrm{CH}_{2} \mathrm{Cl}_{2} / 2$-propanol $=100 / 1$ as eluents) and recrystalized from hexane to afford $196 \mathrm{mg}(55 \%)$ of $(R, R)-7 \mathbf{j}$ as a colorless amorphous solid.

Analytical Data for $(R, R)-7 \mathbf{j}$ :

1 ${ }_{\mathrm{H} \text { NMR: }} \quad\left(500 \mathrm{MHz}, \mathrm{CDCl}_{3}\right)$

7.24-7.20 (m, 8H), 7.18-7.13 (m, 6H), $7.10(\mathrm{~m}, 2 \mathrm{H}), 7.00(\mathrm{~d}, J=8.1,2 \mathrm{H}), 6.94(\mathrm{t}$, $J=7.3,1 \mathrm{H}), 6.91(\mathrm{t}, J=6.6,1 \mathrm{H}), 4.93(\mathrm{~d}, J=8.2,1 \mathrm{H}, \mathrm{NCH}), 4.88(\mathrm{dd}, J=8.2$, 1.4, $1 \mathrm{H}, \mathrm{NCH}), 3.36\left(\mathrm{~m}, 2 \mathrm{H}, \mathrm{C}\left(2^{\prime} / 6^{\prime}\right) \mathrm{H}_{2}\right), 3.14\left(\mathrm{~m}, 2 \mathrm{H}, \mathrm{C}\left(2^{\prime} / 6^{\prime}\right) \mathrm{H}_{2}\right), 1.43(\mathrm{~m}, 4 \mathrm{H}$, $\left.\mathrm{C}\left(3^{\prime}, 5^{\prime}\right) \mathrm{H}_{2}\right), 1.13\left(\mathrm{~m}, 2 \mathrm{H}, \mathrm{C}\left(4^{\prime}\right) \mathrm{H}_{2}\right)$. 
13C NMR: $\quad\left(100 \mathrm{MHz}, \mathrm{CDCl}_{3}\right)$

140.24 (d, $J=3.1$, ipso-Ph), 140.22 (d, $J=6.1$, ipso-Ph), 138.3 (d, $J=8.8$, ipso$\mathrm{Ph}), 138.2$ (d, $J=6.1$, ipso-Ph), 128.9, 128.6, 128.5, 128.0, 127.7, 127.5, 123.2, 122.2, $121.8(\mathrm{~d}, J=3.8, o-\mathrm{Ph}), 119.5(\mathrm{~d}, J=4.6, o-\mathrm{Ph}), 67.7(\mathrm{~d}, J=9.9, \mathrm{CH}), 66.7$ $(\mathrm{d}, J=11.4, \mathrm{CH}), 45.3\left(\mathrm{~d}, J=3.1, \mathrm{C}\left(2^{\prime}, 6^{\prime}\right)\right), 25.4\left(\mathrm{~d}, J=4.6, \mathrm{C}\left(3^{\prime}, 5^{\prime}\right)\right), 24.3$ $\left(\mathrm{C}\left(4^{\prime}\right)\right)$.

31P NMR: $\quad\left(162 \mathrm{MHz}, \mathrm{CDCl}_{3}\right)$

17.25

IR: $\quad(\mathrm{KBr})$

$3063(\mathrm{w}), 3029$ (w), 3010 (w), 2934 (m), 2851 (m), 1630 (w), 1599 (s), 1494 (s), 1455 (m), 1401 (s), 1385 (m), 1340 (m), 1259 (s), 1236 (s), 1165 (m), 1120 (m), 1067 (m), 1030 (m), 965 (s), 917 (w), 793 (w), 757 (m), 734 (m), 698 (m)

MS: $\quad(\mathrm{EI}, 70 \mathrm{eV})$

$494\left(\mathrm{M}^{+}+1,22\right), 493\left(\mathrm{M}^{+}, 57\right), 182(48), 181(100), 180$ (34), 104 (9), 84 (22), 77 (17).

TLC: $\quad R_{f} 0.89$ (EtOAc)

Opt. Rot.: $\quad[\alpha]_{\mathrm{D}}^{22}+174.8\left(c=1.05, \mathrm{CHCl}_{3}\right)$ 
( \pm -1,3,4,5-Tetraphenyl-2-piperidino-1,3,2-diazaphospholidine 2-Oxide ( \pm )-(7j)

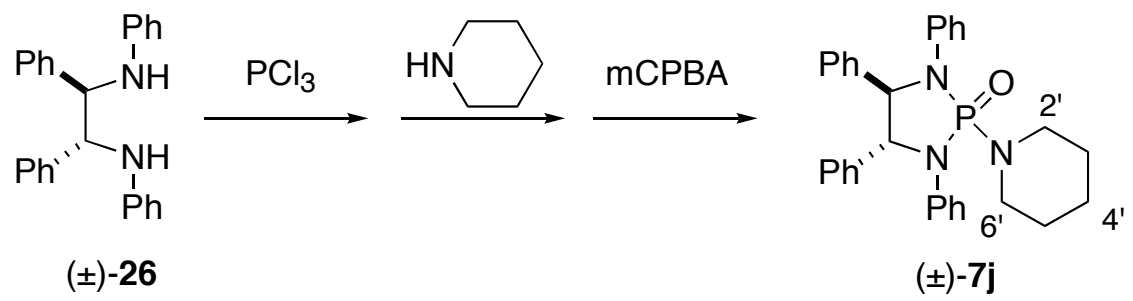

Following Representative Procedure II, from ( \pm )-26 ${ }^{4}(1.09 \mathrm{~g}, 3.0 \mathrm{mmol}), \mathrm{PCl}_{3}(294 \mathrm{~mL}$, $3.3 \mathrm{mmol}, 1.1$ equiv), triethylamine $(0.92 \mathrm{~mL}, 6.6 \mathrm{mmol}, 2.2$ equiv), piperidine $(0.89 \mathrm{~mL}, 9.0$ mmol, 3.0 equiv) and $\mathrm{MCPBA}\left(50 \%, 1.14 \mathrm{~g}, 3.3 \mathrm{mmol}, 1.1\right.$ equiv) in $\mathrm{CH}_{2} \mathrm{Cl}_{2}(15+15+15 \mathrm{~mL})$ was obtained $0.62 \mathrm{~g}(42 \%)$ of $( \pm)-7 \mathbf{j}$ as a colorless amorphous solid after column chromatography ( $\left.\mathrm{SiO}_{2}, \mathrm{CH}_{2} \mathrm{Cl}_{2} / i-\mathrm{PrOH}, 20 / 1\right)$ and recrystallization (toluene/hexane).

Analytical Data for $( \pm)-7 \mathbf{j}$ :

M.W.: $\quad 493.59$

mp: $\quad 196-197{ }^{\circ} \mathrm{C}$ (toluene/hexane)

31 P NMR: $\quad\left(162 \mathrm{MHz}, \mathrm{CDCl}_{3}\right)$

17.30

MS: $\quad(E I, 70 \mathrm{eV})$

494 (M+1, 21), 493 ( $\left.\mathrm{M}^{+}, 50\right), 182$ (100), 180 (39), 104 (10), 84 (25), 77 (18).

TLC: $\quad R_{f} 0.49$ (EtOAc)

Analysis: $\quad \mathrm{C}_{31} \mathrm{H}_{32} \mathrm{~N}_{3} \mathrm{OP}$

$\begin{array}{lllll}\text { Calcd: } \mathrm{C}, \quad 75.44 \% ; & \mathrm{H}, & 6.53 \% ; \mathrm{N}, & 8.51 \% ; \mathrm{P}, & 6.28 \% \text {. }\end{array}$

Found: $\mathrm{C}, \quad 75.68 \% ; \quad \mathrm{H}, \quad 6.51 \% ; \mathrm{N}, \quad 8.49 \% ; \mathrm{P}, \quad 6.25 \%$. 


\section{(+)-(4S,5S)-1,3-Bis-(1-naphthyl)-4,5-diphenyl-2-piperidino-1,3,2-diazaphospholidine 2-}

\section{Oxide $(S, S)-7 k$.}<smiles>c1ccc([C@H](Nc2cccc3ccccc23)[C@H](Nc2cccc3ccccc23)c2ccccc2)cc1</smiles>

$(S, S)-27$

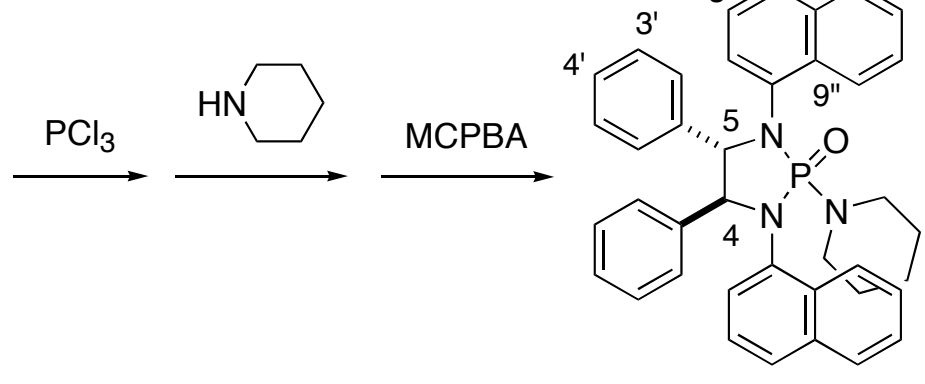

$(S, S)-7 k$

Following Representative Procedure II, to a solution of phosphorus trichloride $(100 \mu \mathrm{L}$, 1.14 mmol, 1.1 equiv) in $\mathrm{CH}_{2} \mathrm{Cl}_{2}(10 \mathrm{~mL})$ was added the solution of the diamine $(S, S)-27^{4}(482$ mg, 1.04 mmol, 1.0 equiv) in $\mathrm{CH}_{2} \mathrm{Cl}_{2}(4 \mathrm{~mL})$ via a cannula at $-78{ }^{\circ} \mathrm{C}$ followed by triethylamine (363 $\mu \mathrm{L}, 3.12 \mathrm{mmol}, 3.0$ equiv). The colorless solution was then slowly warmed to $\mathrm{rt}$ and the solution turned light green-yellow. The solution was stirred at $\mathrm{rt}$ for $6 \mathrm{~h}$ and then was cooled back to $-78{ }^{\circ} \mathrm{C}$. Piperidine $(310 \mu \mathrm{L}, 3.12 \mathrm{mmol}, 3.0$ equiv) was added and then the solution was allowed to warm to $\mathrm{rt}$ and was stirred overnight. A solution of MCPBA (50\%, $431 \mathrm{mg}, 1.25$ mmol, 1.2 equiv) in $\mathrm{CH}_{2} \mathrm{Cl}_{2}(2 \mathrm{~mL})$ was added and the mixture stirred at $\mathrm{rt}$ for $5 \mathrm{~h}$. The redorange solution was then poured into sat. aq. $\mathrm{NaHCO}_{3}$ solution $(30 \mathrm{~mL})$ and the layers were separated. The aqueous layer was extracted with $\mathrm{CH}_{2} \mathrm{Cl}_{2}(2 \mathrm{X} 20 \mathrm{~mL})$ and combined organic layers were washed with brine $(20 \mathrm{~mL})$, dried $\left(\mathrm{MgSO}_{4}\right)$, and concentrated on a rotavap. The oily residue was then purified by column chromatography $\left(\mathrm{SiO}_{2}\right.$, hexane/EtOAc, 3/1 to 1/1) to give $309 \mathrm{mg}(50 \%)$ of $(S, S)-7 \mathbf{k}$ as an off-white foam, which was recrystallized from toluene/hexane to give white amorphous powder.

Analytical Data for $(S, S)-7 \mathbf{k}$ :

M.W.: $\quad 593.72$

mp: $\quad 188-190{ }^{\circ} \mathrm{C}($ toluene/hexane $)$

1슬 $: \quad\left(500 \mathrm{MHz}, \mathrm{CDCl}_{3}\right)$

mixture of rotamers: $9.54(\mathrm{~d}, J=8.4), 8.82-8.38(\mathrm{br}), 8.32-8.06(\mathrm{br}), 7.96-6.92$ 
(br), 5.64-5.52 (br), 5.57 (d, $J=9.2), 5.24-5.12$ (br), 3.36 -2.72 (br), 1.74-1.62 (br), 1.32-0.36 (br).

${ }^{13}$ C NMR: $\quad\left(125 \mathrm{MHz}, \mathrm{CDCl}_{3}\right)$

mixture of rotamers: 138.8, 137.8, 134.5, 132.6, 131.1, 129.0, 128.6, 128.2, 128.0, 127.8, 127.6, 127.4, 126.6, 126.2, 125.6, 125.0, 124.6, 123.6, 118.1, 71.76, $69.9,46.2,45.7,26.0,25.0,24.0,14.1$.

31 $\underline{\text { P NMR: }} \quad\left(202 \mathrm{MHz}, \mathrm{CDCl}_{3}\right)$

mixture of rotamers: 21.29 (br), 20.25 (br), 14.02 (minor).

IR: $\quad\left(\mathrm{CHCl}_{3}\right)$

3091 (w), 3065 (w), 2986 (m), 2968 (m), 2941 (m), 2903 (w), 2884 (w), 2879 (w), 2854 (m), 1596 (m), 1575 (m), 1510 (w), 1497 (w), 1465 (w), 1456 (w), 1444 (w), 1393 (s), 1376 (w), 1363 (w), 1342 (w), 1307 (w), 1281 (m), 1266 (m), 1251 (w), 1237 (w), 1192 (w), 1166 (m), 1151 (w), 1116 (m), 1097 (s), 1072 (s), 1049 (m), 1034 (w), 1026 (m), 967 (s), 945 (m), 915 (w), 854 (w), 839 (w), 820 (w), $699(\mathrm{w}), 605(\mathrm{w})$.

MS: $\quad(\mathrm{EI}, 70 \mathrm{eV})$

594 (M+1, 10), $593\left(\mathrm{M}^{+}, 23\right), 446$ (17), 232 (24), 231 (100), 230 (13), 91 (14), 84 (10), 57 (16), $56(12)$.

Opt. Rot.: $\quad[\alpha]_{\mathrm{D}}^{22}-356\left(\mathrm{CHCl}_{3}, \mathrm{c}=1.01\right)$

TLC: $\quad R_{f} 0.33$ (hexane / EtOAc, 1/1).

Analysis: $\quad \mathrm{C} 39 \mathrm{H} 36 \mathrm{~N} 3 \mathrm{OP}$

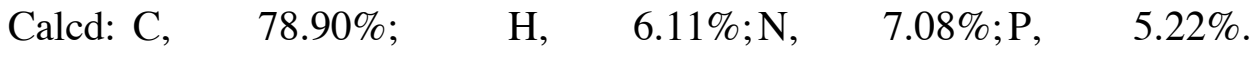

Found: $\mathrm{C}, \quad 78.86 \% ; \quad \mathrm{H}, \quad 6.26 \% ; \mathrm{N}, \quad 6.91 \% ; \mathrm{P}, \quad 5.18 \%$. 

$(S, S)-7 \mathrm{~m}$

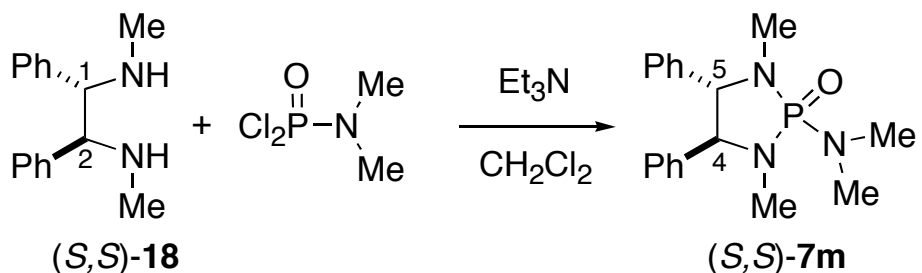

Following Representative Procedure I, from triethylamine $(0.21 \mathrm{~mL}, 0.154 \mathrm{~g}, 1.53$ mmol), $(S, S)-18^{2}(0.146 \mathrm{~g}, 0.61 \mathrm{mmol})$ and $N, N$-dimethylphosphoramic dichloride $(0.073 \mathrm{~mL}$, $0.099 \mathrm{~g}, 0.61 \mathrm{mmol})$ in $\mathrm{CH}_{2} \mathrm{Cl}_{2}(5+1+1 \mathrm{~mL})$, was obtained $0.121 \mathrm{~g}(60 \%)$ of $(S, S)-7 \mathbf{m}$ as a white solid after chromatography $\left(\mathrm{SiO}_{2}, \mathrm{EtOAc} / i-\mathrm{PrOH} 10 / 1\right)$. An analytical sample was obtained by recrystallization from hexane.

Analytical data for $(S, S)-7 \mathbf{m}$ :

mp: $\quad 161-163{ }^{\circ} \mathrm{C}$ (hexane)

${ }^{1} \underline{\text { H NMR: }} \quad\left(400 \mathrm{MHz}, \mathrm{CDCl}_{3}\right)$

7.30 - 7.25 (m, 6H, CH(Ar)), 7.14 - 7.10 (m, 2H, CH(Ar)), 7.08 - 7.05 (m, 2H, $\mathrm{CH}(\mathrm{Ar})$ ), 3.94 (dm $J=9.0, \mathrm{HCPh}), 3.90$ (d, $J=8.8, \mathrm{HCPh}), 2.88$ (d, $J=9.5,6 \mathrm{H}$, $\left.\left(\mathrm{CH}_{3}\right)_{2} \mathrm{~N}\right), 2.37\left(\mathrm{~d}, J=9.0, \mathrm{CH}_{3} \mathrm{~N}\right), 2.35\left(\mathrm{~d}, J=10.3, \mathrm{CH}_{3} \mathrm{~N}\right)$

${ }^{13} \underline{\mathrm{C} \mathrm{NMR}}: \quad\left(100 \mathrm{MHz}, \mathrm{CDCl}_{3}\right)$

138.3 (C(Ar)), 138.2(C(Ar)), 128.4 (CH(Ar)), 128.4 (CH(Ar)), 128.0 (CH(Ar)), $128.0(\mathrm{CH}(\mathrm{Ar})), 127.9(\mathrm{CH}(\mathrm{Ar})), 127.7(\mathrm{CH}(\mathrm{Ar})), 72.3(\mathrm{~d}, J=11.4)$ and $70.7(\mathrm{~d}, J$ $=10.7)\left((\mathrm{C}(4), \mathrm{C}(5)), 37.0\left(\mathrm{~d}, J=4.6,\left(\mathrm{CH}_{3}\right)_{2} \mathrm{~N}\right), 30.2\left(\mathrm{~d}, J=2.3, \mathrm{CH}_{3} \mathrm{~N}\right), 29.2(\mathrm{~d}\right.$, $\left.J=4.6, \mathrm{CH}_{3} \mathrm{~N}\right)$

31 $\underline{\text { P NMR: }} \quad\left(162 \mathrm{MHz}, \mathrm{CDCl}_{3}\right)$

29.61

IR: $\quad(\mathrm{KBr})$

3470 (w), 3210 (w), 3027 (w), 2940 (w), 2805 (w), 1491 (m), 1455 (m), 1291 (m), 1244 (s), 1202 (s), 1152 (s), 1071 (m), 984 (s), 791 (s), 745 (s) 
MS: $\quad(70 \mathrm{eV})$

$329\left(\mathrm{M}^{+}, 85\right), 210$ (38), 166 (27), 165 (28), $120(28), 119(56), 118(100)$

TLC: $\quad R_{f} 0.23(\mathrm{EtOAc} / i-\mathrm{PrOH}, 10 / 1)$

Opt. Rot.: $\quad[\alpha]_{D}^{22}-5.10\left(\mathrm{c}=1.02, \mathrm{CHCl}_{3}\right)$

Analysis: $\quad$ Calcd for $\mathrm{C}_{18} \mathrm{H}_{24} \mathrm{~N}_{3} \mathrm{OP}$

Calcd: $\quad$ C, 65.64; H, 7.34; N, 12.76; P, 9.40.

Found: $\quad$ C, 65.68; $\quad \mathrm{H}, 7.30 ; \quad \mathrm{N}, 12.65 ; \quad \mathrm{P}, 9.34$.

(4S,5S)-1,3-Dimethyl-4,5-diphenyl-2-di-n-propylamino-1,3,2-diazaphospholidine 2-Oxide $(S, S)-7 \mathrm{n}$<smiles>CNC(N[14CH3])C(c1ccccc1)c1ccccc1</smiles>

$(S, S)-18$

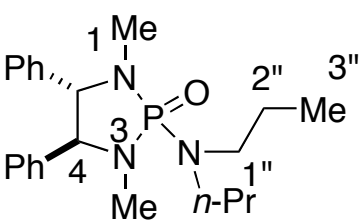

$(S, S)-7 n$

Following Representative Procedure I, from triethylamine $(0.42 \mathrm{~mL}, 3.0 \mathrm{mmol}, 3.0$ equiv), $(S, S)-18^{2}(0.248 \mathrm{~g}, 1.0 \mathrm{mmol})$ and $N, N$-dipropylphosphoramic dichloride $(0.218 \mathrm{~mL}, 1.0$ mmol, 1.0 equiv) in $\mathrm{CH}_{2} \mathrm{Cl}_{2}(20 \mathrm{~mL})$ was obtained $0.179 \mathrm{~g}(46 \%)$ of $7 \mathbf{n}$ as a thick oil after column chromatography $\left(\mathrm{SiO}_{2}\right.$, EtOAc/i-PrOH 10/1).

Analytical Data for $(S, S)-7 \mathbf{n}$ :

$1_{\text {H NMR: }} \quad\left(500 \mathrm{MHz}, \mathrm{CDCl}_{3}\right)$

7.28-7.20 (m, 6 H, Ar), 7.18-7.12 (d, 2 H, Ar), 7.04-6.98 (d, Ar), 3.94 (d, $J=8.6$, 1H, HC(4)), 3.90 (d, $J=8.6,1 \mathrm{H}, \mathrm{HC}(5)), 3.22-3.12$ (m, 2 H, H2C(1")), 3.04-2.96 (m, $\left.2 \mathrm{H}, \mathrm{H}_{2} \mathrm{C}(1 ")\right), 2.35$ (d, $\left.J=9.3,3 \mathrm{H}, \mathrm{MeN}(1)\right), 2.32(\mathrm{~d}, J=11.5,3 \mathrm{H}$, $\operatorname{MeN}(3)), 1.76-1.58$ (m, 4 H, $\left.\mathrm{H}_{2} \mathrm{C}(2 ")\right), 0.97$ (t, $\left.J=7.3,6 \mathrm{H}, \mathrm{Me}(3 ")\right)$

31 $\underline{\text { P NMR: }} \quad\left(162 \mathrm{MHz}, \mathrm{CDCl}_{3}\right)$

27.74

MS: $\quad(E I, 70 \mathrm{eV})$ $386\left(\mathrm{M}^{+}+1,13\right), 385\left(\mathrm{M}^{+}, 34\right), 357$ (29), 356 (100), 286 (22), 285 (96), 251 (14), 
166 (40), 165 (10), 133 (53), 132 (63), 120 (24), 119 (20), 118 (54), 100 (39), 91 (18), $60(23)$

Analysis: $\quad$ Calcd for $\mathrm{C}_{22} \mathrm{H}_{32} \mathrm{~N}_{3} \mathrm{OP}$ (385.49)

Calcd: $\quad$ C, $68.55 \% ; \quad H, 8.37 \% ; \quad$ N, $10.90 \%$.

Found: $\quad$ C, $68.52 \% ; \quad H, 8.07 \% ; \quad$ N, $10.98 \%$.

(4S,5S)-1,3-Di-(2-propyl)-4,5-diphenyl-2-piperidino-1,3,2-diazaphospholidine 2-Oxide $(S, S)-70)$

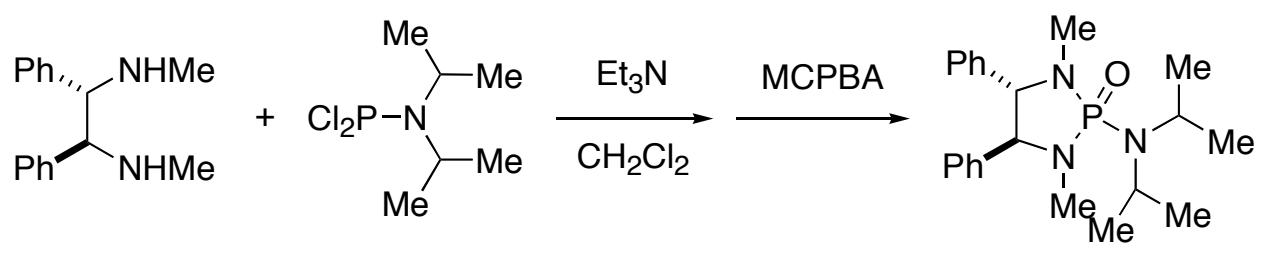

$(S, S)-18$

$(S, S)-70$

Following Representative Procedure III, from $(S, S)-\mathbf{1 8}^{2}$ (483 mg, $\left.2.01 \mathrm{mmol}\right)$, triethylamine (0.62 mL, $4.42 \mathrm{mmol}, 2.2$ equiv) and dimethylaminophosphoric dichloride (0.414 $\mathrm{mL}, 2.01 \mathrm{mmol}, 1.0$ equiv) in $\mathrm{CH}_{2} \mathrm{Cl}_{2}(80+10+10 \mathrm{~mL})$ was obtained $549 \mathrm{mg}(71 \%)$ of $(S, S)-7 \mathbf{o}$ as a white foam after chromatography $\left(\mathrm{SiO}_{2}, \mathrm{TBME} / \mathrm{MeOH}, 49 / 1\right)$.

Analytical Data for $(S, S)-70$ :

M.W.: $\quad 385.49$

1슬 $: \quad\left(500 \mathrm{MHz}, \mathrm{CDCl}_{3}\right)$

7.28-7.22 (m, 6 H, Ph), 7.18-7.04 (m, 2 H, Ph), 7.00-6.96 (m, 2 H, Ph), 3.92 (d, J $=8.8,1 \mathrm{H}, \mathrm{HC}(4)), 3.87(\mathrm{~d}, J=8.8, \mathrm{HC}(5)), 3.50-3.88\left(\mathrm{~m}, 2 \mathrm{H}, \mathrm{HC}\left(1^{\prime}\right)\right), 2.35(\mathrm{~d}, J$ $=9.2,3 \mathrm{H}, \mathrm{MeN}), 2.35(\mathrm{~d}, J=10.3,3 \mathrm{H}, \mathrm{MeN}), 1.42-1.32(\mathrm{~m}, 12 \mathrm{H}, \mathrm{Me}-\mathrm{CH})$.

${ }^{13} \underline{\mathrm{C} \mathrm{NMR}}: \quad\left(125 \mathrm{MHz}, \mathrm{CDCl}_{3}\right)$

$138.8\left(\mathrm{~d}, J=10.1, \mathrm{C}\left(1^{\prime \prime}\right)\right), 138.7$ (d, $\left.J=5.5, \mathrm{C}\left(1^{\prime \prime}\right)\right), 128.4\left(\mathrm{C}\left(3^{\prime \prime}\right)\right), 128.3\left(\mathrm{C}\left(3^{\prime \prime}\right)\right)$, $128.0\left(\mathrm{C}\left(2^{\prime \prime}\right)\right), 127.9\left(\mathrm{C}\left(2^{\prime \prime}\right)\right), 127.8\left(\mathrm{C}\left(4^{\prime \prime}\right)\right), 127.5\left(\mathrm{C}\left(4^{\prime \prime}\right)\right), 72.1$ (d, $J=11.0$, C(4)), 70.9 (d, $J=11.0, \mathrm{C}(5)), 45.8$ (br, C(1')), 30.0 (d, $J=2.8, \mathrm{MeN}), 29.4$ (d, $J$ = 5.5, MeN), 24.0 (br, C(2')). 
31

28.12

MS: $\quad(E I, 70 \mathrm{eV})$

$385\left(\mathrm{M}^{+}, 20\right), 371$ (12), 370 (44), 343 (15), 342 (60), 286 (26), 285 (100), 266

(11), 167 (11), 166 (56), 165 (11), 120 (30), 119 (30), 118 (77), 105 (24), 100

(61), 91 (12), 77(21), 73 (83), 60 (30), 59 (17), 57 (36)

HRMS (EI)

Calcd. for $\mathrm{C}_{22} \mathrm{H}_{32} \mathrm{~N}_{3} \mathrm{OP} \quad 385.2283$;

Found: 385.2284 .

TLC: $\quad R_{f} 0.42(\mathrm{TBME} / \mathrm{MeOH}, 19 / 1)$

$(R, R)$-1,3-Dimethyl-4,5-diphenyl-2-diphenylamino-1,3,2-diazaphospholidine 2-Oxide $(R, R)$ 7 p.

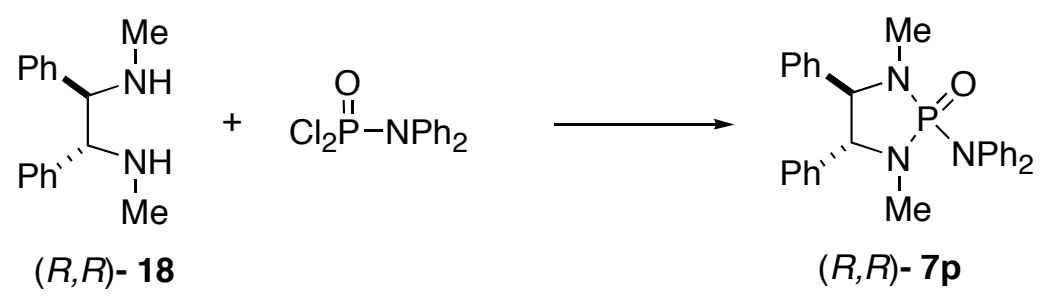

Following Representative Procedure I, to a solution of triethylamine $(0.44 \mathrm{~mL}, 3.16$ $\mathrm{mmol})$ in $30 \mathrm{~mL}$ of dry $\mathrm{CH}_{2} \mathrm{Cl}_{2}$ were added solutions of $(R, R)-\mathbf{1 8}^{7}(314 \mathrm{mg}, 1.31 \mathrm{mmol})$ in 15 $\mathrm{mL}$ of dry $\mathrm{CH}_{2} \mathrm{Cl}_{2}$ and diphenylaminophosphorus dichloride $(450 \mathrm{mg}, 1.57 \mathrm{mmol})$ in $15 \mathrm{~mL}$ of dry $\mathrm{CH}_{2} \mathrm{Cl}_{2}$ simultaneously and dropwise over $30 \mathrm{~min}$ at reflux under nitrogen. The solution was heated to reflux for $36 \mathrm{~h}$ then was concentrated under reduced pressure to give a residue, which was purified by column chromatography on silica gel $\left(\mathrm{CH}_{2} \mathrm{Cl}_{2}, \mathrm{CH}_{2} \mathrm{Cl}_{2} / 2\right.$-propanol, $100 / 2$ then 100/4) affording $372 \mathrm{mg}(63 \%)$ of $(R, R)-7 \mathbf{p}$ as a colorless amorphous solid.

Data for $(R, R)-7 \mathbf{p}$ :

1 $\mathrm{H} \mathrm{NMR}: \quad\left(400 \mathrm{MHz}, \mathrm{CDCl}_{3}\right)$

7.44-7.35 (m, 8H, o, $m-\mathrm{Ph}$ ), 7.27-7.17 (m, 6H, $\left.m, p-\mathrm{Ph}^{\prime}, p-\mathrm{Ph}, p-\mathrm{Ph} "\right), 7.12-7.08$ 
(m, 4H, $\left.o-\mathrm{Ph}^{\prime}, m-\mathrm{Ph} "\right), 6.44$ (d, $\left.J=8.3,2 \mathrm{H}, o-\mathrm{Ph}^{\prime \prime}\right), 3.99$ (d, $\left.J=8.9,1 \mathrm{H}, \mathrm{NCH}\right)$, $3.71(\mathrm{~d}, J=8.9,1 \mathrm{H}, \mathrm{NCH}), 2.56(\mathrm{~d}, J=9.5,3 \mathrm{H}, \mathrm{CH} 3), 2.46(\mathrm{~d}, J=10.3,3 \mathrm{H}$, $\left.\mathrm{CH}_{3}\right)$.

13C NMR: $\quad\left(125 \mathrm{MHz}, \mathrm{CDCl}_{3}\right)$

145.3 (d, $J=4.6$, ipso-Ph), 137.7 (ipso-Ph), 137.6 (d, $J=4.6$, ipso-Ph), 129.1, $128.5,128.23,128.16,128.09,127.99$ (d, $J=2.8), 127.95,127.87,125.4,71.2$ (d, $J=10.0, \mathrm{NCH}), 71.0(\mathrm{~d}, J=11.0, \mathrm{NCH}), 29.6\left(\mathrm{~d}, J=3.7, \mathrm{CH}_{3}\right), 29.1(\mathrm{~d}, J=5.5$, $\left.\mathrm{CH}_{3}\right)$

31P NMR: $\quad\left(162 \mathrm{MHz}, \mathrm{CDCl}_{3}\right)$

20.76

IR: $\quad(\mathrm{KBr})$

3088 (w), 3062 (w), 3032 (w), 2974 (w), 2930 (m), 2904 (m), 2856 (m), 2815 (w), 1590 (m), 1489 (s), 1455 (m), 1401 (m), 1339 (w), 1300 (m), 1280 (m), 1257 (s), 1211 (s), 1166 (s), 1074 (m), 1033 (s), 1016 (s), 991 (s), 915 (w), 901 (w), $891(\mathrm{w}), 830(\mathrm{w}), 814(\mathrm{w}), 777(\mathrm{~m}), 746$ (s), $700(\mathrm{~s}), 632(\mathrm{~m}), 626(\mathrm{w}), 613(\mathrm{w})$, $555(\mathrm{~s})$

Opt. Rot.: $\quad[\alpha]_{\mathrm{D}}^{22}+25.7\left(c=0.65, \mathrm{CHCl}_{3}\right)$

TLC: $\quad R_{f} 0.73$ (EtOAc)

Analysis: $\quad \mathrm{C}_{28} \mathrm{H}_{28} \mathrm{~N}_{3} \mathrm{OP}(453.52)$

Calcd.: C, 74.15; $\quad$ H, 6.22; $\quad$ N, 9.27; $\quad$ P, 6.83

Found: C, $\quad \mathrm{H}, ; \quad \mathrm{N}, ; \quad \mathrm{P}$, 

$(S, S)-7 q$.

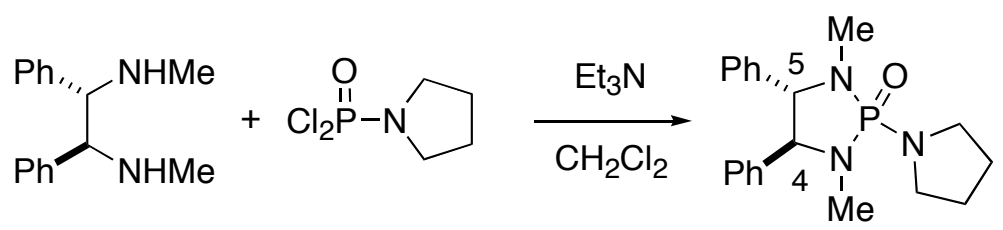

$(S, S)-18$

$(S, S)-7 q$

Following Representative Procedure I, from triethylamine (0.55 mL, $3.93 \mathrm{mmol}, 2.5$ equiv), $(S, S)-1^{2}(0.377 \mathrm{~g}, 1.57 \mathrm{mmol})$ and 1-pyrrolidinylphosphoramic dichloride $(0.295 \mathrm{~g}, 1.57 \mathrm{mmol}$, 1.0 equiv) in $\mathrm{CH}_{2} \mathrm{Cl}_{2}(20+8+8 \mathrm{~mL})$ was obtained $0.34 \mathrm{~g}(61 \%)$ of $(S, S)-7 \mathbf{q}$ as white crystals after column chromatography ( $\mathrm{SiO}_{2}, \mathrm{EtOAc} / \mathrm{i}-\mathrm{PrOH}$ 9/1) and recrystallization (hexane), mp: 105-106 ${ }^{\circ} \mathrm{C}$ (hexane); TLC $R_{f}=0.32$ (EtOAc/i-PrOH, 10/1). Anal. Calcd for $\mathrm{C}_{20} \mathrm{H}_{26} \mathrm{~N}_{3} \mathrm{OP}$ (355.42): C, 67.5; H, 7.37; N, 11.82. Found: C, 67.36; H, 7.13; N, 11.78.

\section{(S)-1,3-Dimethyl-2-methyl(1-phenylethyl)amino-1,3,2-diazaphospholidine 2-Oxide (8a)}

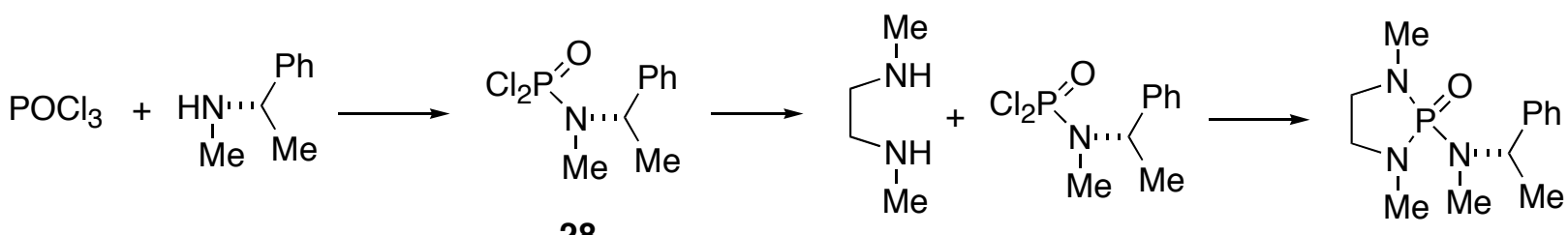

28

$8 a$

A solution of $(S)$-1-methylamino-1-phenylethane $(7.3 \mathrm{~mL}, 50 \mathrm{mmol})$ in $15 \mathrm{~mL}$ of dry diethyl ether was added to a solution of $\mathrm{POCl}_{3}(14 \mathrm{~mL}, 150 \mathrm{mmol})$ in $25 \mathrm{~mL}$ of dry diethyl ether, dropwise through an addition funnel at $0{ }^{\circ} \mathrm{C}$ under nitrogen. After completion of the addition, the mixture was stirred at rt overnight. The solvent was distilled off, the mixture was heated at 130$140{ }^{\circ} \mathrm{C}$ (bath temperature) to reflux for $20 \mathrm{~h}$, the $\mathrm{POCl}_{3}$ was distilled off under atmospheric pressure then the product was distilled under reduced pressure to give $9.35 \mathrm{~g}(74 \%)$ of 28 as a colorless liquid (bp $150{ }^{\circ} \mathrm{C} / 1.1 \mathrm{mmHg}$; ABT) which solidified upon cooling. 
Data for 28:

1

7.37-7.31 (m, 5H, Ph), 5.39 (dq, $\left.J=11.0,7.1, \mathrm{CHCH}_{3}\right), 2.55(\mathrm{~d}, J \mathrm{PH}=16.1$, $\mathrm{NCH} 3), 1.63\left(\mathrm{~d}, J=6.8, \mathrm{CHCH}_{3}\right)$

31P NMR: $\quad\left(162 \mathrm{MHz}, \mathrm{CDCl}_{3}\right)$

18.19

To a solution of triethylamine $(3.5 \mathrm{~mL}, 25 \mathrm{mmol})$ in $200 \mathrm{~mL}$ of dry $\mathrm{CH}_{2} \mathrm{Cl}_{2}$ were added solutions of $N, N^{\prime}$-dimethylethylenediamine $(1.06 \mathrm{~mL}, 10 \mathrm{mmol})$ in $25 \mathrm{~mL}$ of dry $\mathrm{CH}_{2} \mathrm{Cl}_{2}$ and (S)-methyl(1-phenylethyl)aminophosphorus dichloride 18 (2.52 g, $10 \mathrm{mmol})$ in $25 \mathrm{~mL}$ of dry $\mathrm{CH}_{2} \mathrm{Cl}_{2}$ simultaneously over $2 \mathrm{~h}$ using a syringe pump at reflux under nitrogen. The solution was heated to reflux for $42 \mathrm{~h}$ then was concentrated under reduced pressure to give a residue, which was purified by column chromatography on silica gel (ethyl acetate/2-propanol, 10/1, 10/2, then 10/3). Distillation under reduced pressure (bp 160-165 ${ }^{\circ} \mathrm{C} / 0.1 \mathrm{mmHg}$; ABT) afforded $2.139 \mathrm{~g}(80 \%)$ of $\mathbf{8 a}$ as hygroscopic colorless crystals. Analytical sample was obtained by further recrystalization from hexane.

Analytical Data for 8a:

mp: $\quad 51-52{ }^{\circ} \mathrm{C}$ (hexane)

1

$7.41(\mathrm{~d}, J=7.1,2 \mathrm{H}, o-\mathrm{Ph}), 7.32(\mathrm{t}, J=7.5,2 \mathrm{H}, m-\mathrm{Ph}), 7.23(\mathrm{t}, J=7.3, p-\mathrm{Ph}), 5.05$ (dq, $\left.J=8.8,7.1, \mathrm{CHCH}_{3}\right), 3.18\left(\mathrm{~m}, 2 \mathrm{H}, \mathrm{CH}_{2}\right), 3.11\left(\mathrm{~m}, 2 \mathrm{H}, \mathrm{CH}_{2}\right), 2.57$ (d, $J=$ 9.7, 3H, NCH3), 2.49 (d, $\left.J=9.7,3 \mathrm{H}, \mathrm{NCH}_{3}\right), 2.27\left(\mathrm{~d}, J=10.2,3 \mathrm{H}, \mathrm{NCH}_{3}\right), 1.52$ (d, $\left.J=7.1,3 \mathrm{H}, \mathrm{CHCH}_{3}\right)$.

13 $\mathrm{C} \mathrm{NMR:} \quad\left(100 \mathrm{MHz}, \mathrm{CDCl}_{3}\right)$

142.3 (d, $J=4.6$, ipso-Ph), 128.0 (o/m-Ph), 127.5 (o/m-Ph), 126.8 (p-Ph), 53.2 (d, $\left.J=4.6, C \mathrm{HCH}_{3}\right), 47.3\left(\mathrm{~d}, J=12.2, \mathrm{CH}_{2}\right), 31.43\left(\mathrm{~d}, J=6.9, \mathrm{NCH}_{3}\right), 31.38(\mathrm{~d}, J=$ 6.9, $\left.\mathrm{NCH}_{3}\right), 26.6\left(\mathrm{~d}, J=5.3, \mathrm{NCH}_{3}\right), 17.5\left(\mathrm{~d}, J=1.5, \mathrm{CHCH}_{3}\right)$

31P NMR: $\quad\left(162 \mathrm{MHz}, \mathrm{CDCl}_{3}\right)$ 
IR: $\quad\left(\mathrm{CHCl}_{3}\right)$

$3104(w), 3088$ (w), 3017 (w), 2979 (s), 2943 (m), 2854 (w), $2819(w), 1494(w)$, 1481 (w), 1470 (w), 1452 (w), 1376 (m), 1352 (s), 1265 (m), 1229 (s), 1211 (s), 1196 (s), 1167 (s), 1038 (m), 1027 (m), 1005 (m), 995 (m), 948 (s), 889 (w), 702 (m), $662(\mathrm{~m}), 518(\mathrm{~m})$

MS: $\quad(E I, 70 \mathrm{eV})$

$267\left(\mathrm{M}^{+}, 38\right), 252$ (22), 135 (13), 134 (100), 133 (91), 105 (13), 90 (8), 77 (8)

Opt. Rot.: $[\alpha]_{\mathrm{D}}^{22}-1.6\left(c=1.1, \mathrm{CHCl}_{3}\right)$

TLC: $\quad R_{f} 0.58(\mathrm{EtOAc} / i-\mathrm{PrOH}, 9 / 1)$

Analysis: $\quad \mathrm{C}_{13} \mathrm{H}_{22} \mathrm{~N}_{3} \mathrm{OP}(267.31)$

Calcd.: C, 58.41\%; H, 8.30\%; N, $15.72 \% ; \quad \mathrm{P}, 11.59 \%$.

Found: C, 58.39\%; H, 8.38\%; N, 15.77\%; P, $11.37 \%$.

(S)-1,3-Diphenyl-2-methyl(1-phenylethyl)amino-1,3,2-diazaphospholidine 2-Oxide (8b)

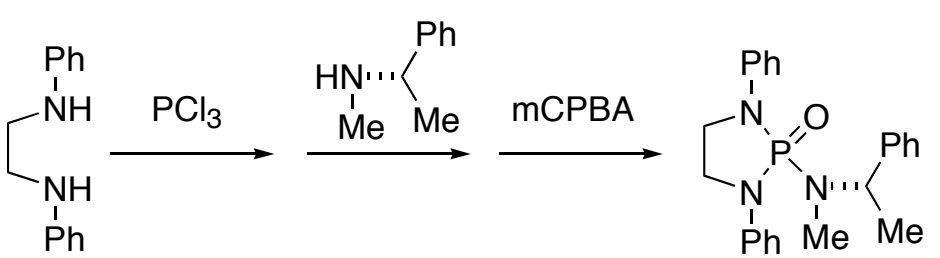

$8 b$

Following Representative Procedure II, to a stirred cold $\left(-78^{\circ} \mathrm{C}\right)$ solution of $\mathrm{PCl}_{3}(0.95$ $\mathrm{mL} ; 11 \mathrm{mmol}$ ) in $20 \mathrm{~mL}$ of dry $\mathrm{CH}_{2} \mathrm{Cl}_{2}$ was added a solution of $N, N^{\prime}$-diphenylethylenediamine $(2.12 \mathrm{~g}, 10 \mathrm{mmol})$ in $20 \mathrm{~mL}$ of dry $\mathrm{CH}_{2} \mathrm{Cl}_{2}$ through a dropping funnel under nitrogen. Then triethylamine $(3.5 \mathrm{~mL} ; 25 \mathrm{mmol})$ was added at the same temperature. The mixture was warmed to room temperature and was stirred for $24 \mathrm{~h}$. To this mixture was added $40 \mathrm{~mL}$ of dry diethyl ether and the precipitates were filtered off and washed with dry ether $(40 \mathrm{~mL})$. The combined filtrate and washings were concentrated under reduced pressure to remove the excess reagents ( $\mathrm{PCl}_{3}$ and triethylamine).

The residue was dissolved in $10 \mathrm{~mL}$ of dry $\mathrm{CH}_{2} \mathrm{Cl}_{2}$ and stirred, to which was added a mixture of $(S)$-1-methylamino-1-phenylethane $(1.45 \mathrm{~mL}, 10 \mathrm{mmol})$ and triethylamine $(4.2 \mathrm{~mL}$, $30 \mathrm{mmol}$ ) at $0{ }^{\circ} \mathrm{C}$, and the mixture was stirred for $18 \mathrm{~h}$ at $\mathrm{rt}$ under nitrogen. After addition of dry 
ether $(30 \mathrm{~mL})$, the suspension was filtered to remove the precipitates and condensed under reduced pressure.

The residue was dissolved in $10 \mathrm{~mL}$ of $\mathrm{CH}_{2} \mathrm{Cl}_{2}$, and a solution of $m$-chloroperbenzoic acid $(50 \%, 3.45 \mathrm{~g}, 10 \mathrm{mmol})$ in $15 \mathrm{~mL}$ of $\mathrm{CH}_{2} \mathrm{Cl}_{2}$ was added dropwise at $0{ }^{\circ} \mathrm{C}$. The mixture was allowed to warm to room temperature and was stirred for $24 \mathrm{~h}$. Then sat. aq. $\mathrm{NaHCO}_{3}$ solution $(25 \mathrm{~mL})$ was added with stirring. The aqueous layer was separated and extracted with $\mathrm{CH}_{2} \mathrm{Cl}_{2}$ (2 X $25 \mathrm{~mL})$. The combined organic solutions were washed with sat. aq. NaHCO3 solution (25 mL) than was dried over $\mathrm{MgSO}_{4}$, filtered, and evaporated to give a crude material, which was purified by column chromatography on silica gel (benzene/ $\mathrm{CH}_{2} \mathrm{Cl}_{2} / 2$-propanol, 30/20/1 as eluent). Recrystallization from $\mathrm{CH}_{2} \mathrm{Cl}_{2}$ /hexane afforded $1.96 \mathrm{~g}(50 \%)$ of $\mathbf{8 b}$ as colorless fine needles. An analytical sample was obtained by sublimation at $0.05 \mathrm{mmHg}$.

Analytical Data for $\mathbf{8 b}$ :

mp: $\quad 204-205^{\circ} \mathrm{C}$ (sublimation)

1H NMR: $\quad\left(400 \mathrm{MHz}, \mathrm{CDCl}_{3}\right)$

$7.32(\mathrm{t}, J=7.8,2 \mathrm{H}, m-\mathrm{Ph}), 7.25(\mathrm{t}, J=7.8,2 \mathrm{H}, m-\mathrm{Ph}), 7.22(\mathrm{~d}, J=7.8,2 \mathrm{H}, o-$ $\mathrm{Ph}), 7.16$ (d, $\left.J=7.6,2 \mathrm{H}, o-\mathrm{Ph}^{\prime}\right), 7.13-7.07$ (m, 3H, $\left.m, p-\mathrm{Ph}^{\prime \prime}\right), 7.04-6.97$ (m, 4H, p-Ph, o-Ph", $p$-Ph'), $4.84\left(\mathrm{dq}, J=10.0,7.1, \mathrm{CHCH}_{3}\right), 3.94-3.76(\mathrm{~m}, 4 \mathrm{H}$, $\left.\mathrm{CH}_{2} \mathrm{CH}_{2}\right), 2.51\left(\mathrm{~d}, J=10.5,3 \mathrm{H}, \mathrm{NCH}_{3}\right), 1.20\left(\mathrm{~d}, J=7.1,3 \mathrm{H}, \mathrm{CHCH}_{3}\right)$.

\section{C NMR: $\quad\left(100 \mathrm{MHz}, \mathrm{CDCl}_{3}\right)$}

141.6 (d, $J=6.6$, ipso-Ph), 140.7 (d, $J=4.6$, ipso- $\mathrm{Ph}), 129.18$ ( $m-\mathrm{Ph}), 129.15$ ( $m$ $\mathrm{Ph}), 127.9$ (o/m-Ph'), 127.5 (o/m-Ph'), 126.9 (p-Ph'), 121.74 (p-Ph), 121.68 (p-Ph), $117.1(\mathrm{~d}, J=4.6, o-\mathrm{PhN}), 117.0(\mathrm{~d}, J=4.6, o-\mathrm{PhN}), 54.0\left(\mathrm{~d}, J=5.3, C \mathrm{HCH}_{3}\right)$, $43.04\left(\mathrm{~d}, J=12.2, \mathrm{CH}_{2}\right), 43.00\left(\mathrm{~d}, J=12.2, \mathrm{CH}_{2}\right), 27.9\left(\mathrm{~d}, J=5.3, \mathrm{NCH}_{3}\right), 16.8$ $\left(\mathrm{CHCH}_{3}\right)$

31P NMR: $\quad\left(162 \mathrm{MHz}, \mathrm{CDCl}_{3}\right)$

15.31

IR: $\quad(\mathrm{KBr})$

3057 (w), 3030 (w), 2981 (w), 2941 (w), 2883 (w), 2811 (w), 1599 (s), 1581 (m), 1499 (s), 1482 (s), 1453 (m), 1400 (m), 1384 (m), 1379 (s), 1363 (m), 1348 (m), 
1317 (m), 1281 (s), 1231 (s), 1207 (s), 1190 (m), 1165 (s), 1135 (s), 1102 (w), 1066 (w), 1048 (m), 1033 (m), 994 (s), 949 (s), 877 (w), 777 (m), 749 (s), 734 (m), $702(\mathrm{~s}), 688(\mathrm{~s}), 616(\mathrm{~m}), 586(\mathrm{w}), 530(\mathrm{~m})$

MS: $\quad(\mathrm{EI}, 70 \mathrm{eV})$

$391\left(\mathrm{M}^{+}, 29\right), 376$ (13), 258 (14), 257 (46), 152 (23), 135 (10), 134 (100), 119 (13), 106 (42), 105 (37), 104 (20), 77 (19).

Opt. Rot.: $\quad[\alpha]_{\mathrm{D}}^{22}+1.7\left(c=1.1, \mathrm{CHCl}_{3}\right)$

TLC: $\quad R f 0.87$ (EtOAc)

Analysis: $\quad \mathrm{C}_{23} \mathrm{H}_{26} \mathrm{~N}_{3} \mathrm{OP}(391.45)$

Calcd.: C, 70.57\%; H, 6.69\%; N, 10.73\%; P, $7.91 \%$.

Found: C, 70.35\%; H, 6.56\%; N, $10.79 \% ; \quad \mathrm{P}, 7.86 \%$.

\section{1,3-Dimethyl-2-piperidino-1,3,2-diazaphospholidine 2-Oxide (11a)}

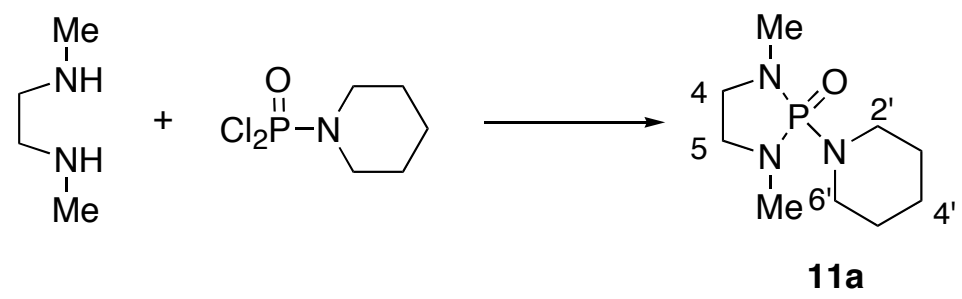

Following Representative Procedure I, to a solution of triethylamine $(2.3 \mathrm{~mL}, 17 \mathrm{mmol})$ in $150 \mathrm{~mL}$ of dry $\mathrm{CH}_{2} \mathrm{Cl}_{2}$ were added solutions of $N, N$ '-dimethylethylenediamine $(0.71 \mathrm{~mL} ; 6.7$ $\mathrm{mmol})$ in $30 \mathrm{~mL}$ of dry $\mathrm{CH}_{2} \mathrm{Cl}_{2}$ and piperidinophosphorus dichloride $(1.02 \mathrm{~mL} ; 6.7 \mathrm{mmol})$ in 30 $\mathrm{mL}$ of dry $\mathrm{CH}_{2} \mathrm{Cl}_{2}$ simultaneously over $2 \mathrm{~h}$ using a syringe pump at reflux under nitrogen. The solution was heated to reflux for $40 \mathrm{~h}$ then was concentrated under reduced pressure to give a colorless residue, which was purified by column chromatography on silica gel (ethyl acetate, ethyl acetate/2-propanol, 9/1, and then ethyl acetate/2-propanol, 7/3 as eluents). Distillation of the product, (bp 120-125 ${ }^{\circ} \mathrm{C} / 0.05 \mathrm{mmHg}$; ABT) afforded $1.45 \mathrm{~g}(100 \%)$ of 11a as colorless hygroscopic crystals. An analytical sample was prepared by further recrystalization from hexane.

Analytical Data for 11a:

mp: $\quad 55-56^{\circ} \mathrm{C}$ (hexane) 
1 1 HMR: $\quad\left(400 \mathrm{MHz}, \mathrm{CDCl}_{3}\right)$

$3.11\left(\mathrm{~m}, 2 \mathrm{H}, \mathrm{C}(4 / 5) \mathrm{H}_{2}\right), 3.05-2.96\left(\mathrm{~m}, 6 \mathrm{H}, \mathrm{C}(4 / 5) \mathrm{H}_{2}, \mathrm{C}\left(2^{\prime}, 6^{\prime}\right) \mathrm{H}_{2}\right), 2.49(\mathrm{~d}, J=$ 9.8, 6H, $\left.\mathrm{CH}_{3}\right), 1.51\left(\mathrm{~m}, 2 \mathrm{H}, \mathrm{C}\left(4^{\prime}\right) \mathrm{H}_{2}\right), 1.41\left(\mathrm{~m}, 4 \mathrm{H}, \mathrm{C}\left(3^{\prime}, 5^{\prime}\right) \mathrm{H}_{2}\right)$.

13 $\mathrm{C} \mathrm{NMR}: \quad\left(100 \mathrm{MHz}, \mathrm{CDCl}_{3}\right)$

$47.2(\mathrm{~d}, J=12.2, \mathrm{C}(4,5)), 45.3\left(\mathrm{~d}, J=3.1, \mathrm{C}\left(2^{\prime}, 6^{\prime}\right)\right), 31.5\left(\mathrm{~d}, J=4.6, \mathrm{CH}_{3}\right), 26.7$ $\left(\mathrm{d}, J=3.8, \mathrm{C}\left(3^{\prime}, 5^{\prime}\right)\right), 24.7\left(\mathrm{C}\left(4^{\prime}\right)\right)$.

31P NMR: $\quad\left(162 \mathrm{MHz}, \mathrm{CDCl}_{3}\right)$

26.44

IR: $\left(\mathrm{CHCl}_{3}\right)$ $2979(\mathrm{~m}), 2938$ (s), 2852 (m), 1493 (w), 1482 (w), 1470 (w), $1445(\mathrm{w}), 1376(\mathrm{w})$, 1351 (w), 1338 (w), 1264 (m), 1230 (m), 1191 (m), 1166 (s), 1122 (w), 1068 (m), 1040 (m), 1026 (w), 961 (s), 943 (m), 863 (w), 853 (w), 834 (w), 670 (w), 665 (w), $547(\mathrm{w})$

MS: $\quad(\mathrm{EI}, 70 \mathrm{eV})$

$217\left(\mathrm{M}^{+}, 30\right), 133$ (44), $131(8), 90(8), 85$ (11), 84 (100)

TLC: $\quad R_{f} 0.20$ (EtOAc/i-PrOH, 9/1)

Analysis: $\quad \mathrm{C}_{9} \mathrm{H}_{20} \mathrm{~N} 3 \mathrm{OP}(217.25)$

Calcd.: C, 49.76\%; H, 9.28\%; N, 19.34\%; P, $14.26 \%$.

Found: C, $49.56 \% ; \quad \mathrm{H}, 9.22 \% ; \quad \mathrm{N}, 19.46 \% ; \quad \mathrm{P}, 14.34 \%$.

\section{1,3-Diisopropyl-2-piperidino-1,3,2-diazaphospholidine 2-Oxide (11b)}

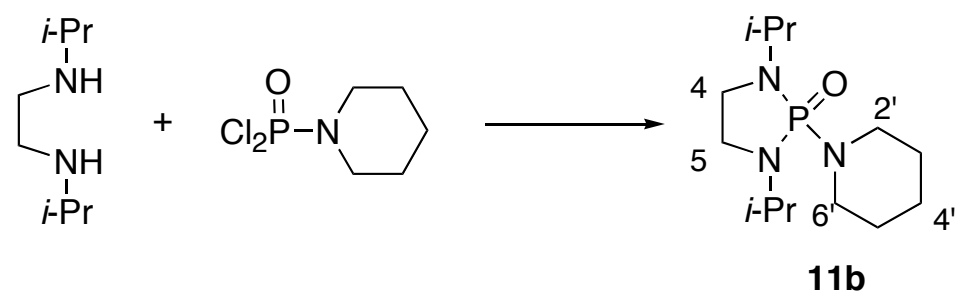

Following Representative Procedure I, to a solution of triethylamine ( $2.4 \mathrm{~mL}, 17.5 \mathrm{mmol})$ in $150 \mathrm{~mL}$ of dry $\mathrm{CH}_{2} \mathrm{Cl}_{2}$ were added solutions of $N, N^{\prime}$-diisopropylethylenediamine $(1.27 \mathrm{~mL}$, $7.0 \mathrm{mmol}$ ) in $30 \mathrm{~mL}$ of dry $\mathrm{CH}_{2} \mathrm{Cl}_{2}$ and piperidinophosphorus dichloride ${ }^{5}(1.07 \mathrm{~mL} ; 7.0 \mathrm{mmol})$ 
in $30 \mathrm{~mL}$ of dry $\mathrm{CH}_{2} \mathrm{Cl}_{2}$ simultaneously over $2 \mathrm{~h}$ using a syringe pump at reflux under nitrogen. The solution was heated to reflux for $36 \mathrm{~h}$ then was concentrated under reduced pressure to give a colorless residue, which was purified by column chromatography on silica gel (ethyl acetate, ethyl acetate/2-propanol, 95/5 then ethyl acetate/2-propanol, 9/1 as eluents). Distillation of the product (bp $145{ }^{\circ} \mathrm{C} / 0.05 \mathrm{mmHg}$; ABT) afforded $949 \mathrm{mg}(50 \%)$ of $\mathbf{1 1 b}$ as hygroscopic colorless crystals. The analytical sample was prepared by further recrystalization from hexane.

Analytical Data for 11b:

mp: $\quad 52-53{ }^{\circ} \mathrm{C}$ (hexane)

1 H NMR: $^{\text {(4) }}\left(400 \mathrm{MHz}, \mathrm{CDCl}_{3}\right)$

3.34 (d sep, $J=8.2 \mathrm{~Hz}, 6.6,2 \mathrm{H}, \mathrm{CH}), 3.09\left(\mathrm{~m}, 2 \mathrm{H}, \mathrm{C}(4 / 5) \mathrm{H}_{2}\right), 2.97(\mathrm{~m}, 6 \mathrm{H}$, $\left.\mathrm{C}(4 / 5) \mathrm{H}_{2}, \mathrm{C}\left(2^{\prime}, 6^{\prime}\right) \mathrm{H}_{2}\right), 1.49$ (m, 2H, C(4') $\left.\mathrm{H}_{2}\right), 1.41$ (m, 4H, C(3' 5') $\left.\mathrm{H}_{2}\right), 1.13$ (d, $\left.J=6.6 \mathrm{~Hz}, 6 \mathrm{H}, \mathrm{CH}_{3}\right), 1.08\left(\mathrm{~d}, J=6.6 \mathrm{~Hz}, 6 \mathrm{H}, \mathrm{CH}_{3}\right)$.

13C NMR: $\quad\left(100 \mathrm{MHz}, \mathrm{CDCl}_{3}\right)$

$45.6\left(\mathrm{~d}, J=3.1, \mathrm{C}\left(2^{\prime}, 6^{\prime}\right)\right), 44.1(\mathrm{~d}, J=5.3, \mathrm{CH}), 39.0(\mathrm{~d}, J=13.0, \mathrm{C}(4,5)), 26.7$

$\left(\mathrm{d}, J=4.6, \mathrm{C}\left(3^{\prime}, 5^{\prime}\right)\right), 24.8\left(\mathrm{C}\left(4^{\prime}\right)\right), 21.4\left(\mathrm{~d}, J=2.3, \mathrm{CH}_{3}\right), 21.2\left(\mathrm{~d}, J=4.6, \mathrm{CH}_{3}\right)$.

31P NMR: $\quad\left(162 \mathrm{MHz}, \mathrm{CDCl}_{3}\right)$

22.44

IR: $\quad\left(\mathrm{CDCl}_{3}\right)$

2970 (s), 2937 (s), 2851 (m), 1477 (w), 1464 (w), 1452 (w), 1443 (w), $1401(\mathrm{w})$, 1390 (w), 1365 (w), 1336 (w), 1230 (m), 1222 (m), 1212 (m), 1179 (s), 1167 (m), $1112(\mathrm{~m}), 1064$ (m), 1027 (w), $960(\mathrm{~s}), 862(\mathrm{w}), 698(\mathrm{w}), 669(\mathrm{w}), 561(\mathrm{w})$

MS: $\quad(E I, 70 \mathrm{eV})$

$273\left(\mathrm{M}^{+}, 34\right), 259$ (10), 258 (70), 189 (15), 175 (79), 147 (8), 133 (17), 105 (17), 85 (10), 84 (100), 72 (12)

TLC: $\quad R_{f} 0.71(\mathrm{EtOAc} / i-\mathrm{PrOH}, 9 / 1)$

Analysis: $\quad \mathrm{C}_{13} \mathrm{H}_{28} \mathrm{~N}_{3} \mathrm{OP}(273.36)$

Calcd.: C, $57.12 \% ; \quad \mathrm{H}, 10.32 \% ; \quad \mathrm{N}, 15.37 \% ; \quad \mathrm{P}, 11.33 \%$.

Found: C, 56.82\%; H, 10.46\%; N, 15.56\%; P, $11.21 \%$. 


\section{1,3-Diphenyl-2-piperidino-1,3,2-diazaphospholidine 2-Oxide (11c)}

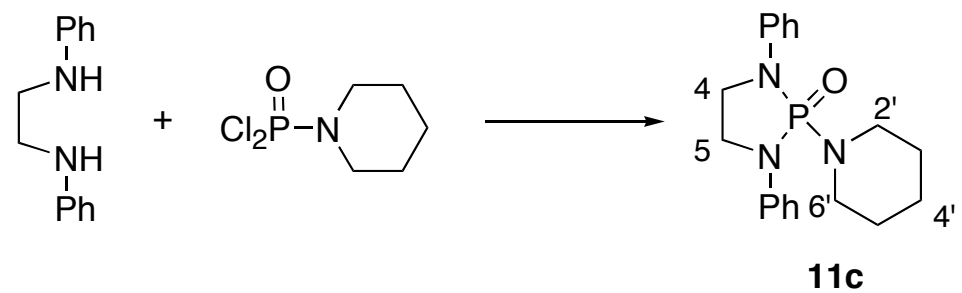

Following Representative Procedure I, to a solution of $N, N^{\prime}$-diphenylethylene diamine $(2.12 \mathrm{~g}, 10 \mathrm{mmol})$ in $20 \mathrm{~mL}$ of dry 1,2-dichloroethane were added triethylamine $(3.5 \mathrm{~mL}, 25$ mmol) and piperidinophosphorus dichloride $5(1.53 \mathrm{~mL}, 10 \mathrm{mmol})$ at r.t. under nitrogen. The mixture was heated to reflux for $19 \mathrm{~h}$ when the mixture solidified. The mixture was filtered with help of $\mathrm{CH}_{2} \mathrm{Cl}_{2}(50 \mathrm{~mL})$ and was washed with $\mathrm{CH}_{2} \mathrm{Cl}_{2}(100 \mathrm{~mL})$. The filtrate and the washings were combined and concentrated under reduced pressure to give tan solid which was purified by column chromatography on silica gel $\left(\mathrm{CH}_{2} \mathrm{Cl}_{2}\right.$ then $\mathrm{CH}_{2} \mathrm{Cl}_{2} / 2$-propanol, 20/1 as eluents). Recrystalization from benzene/hexane afforded $1.741 \mathrm{~g}(51 \%)$ of 11c as colorless fine needles. Analytical Data for 11c:

mp: $\quad 169-171^{\circ} \mathrm{C}$ (benzene-hexane)

1 ${ }_{\mathrm{H} \text { NMR: }} \quad\left(500 \mathrm{MHz}, \mathrm{CDCl}_{3}\right)$

$7.33(\mathrm{t}, J=7.9,4 \mathrm{H}, m-\mathrm{Ph}), 7.17(\mathrm{~d}, J=7.9 \mathrm{~Hz}, 4 \mathrm{H}, o-\mathrm{Ph}), 7.00(\mathrm{t}, J=7.3 \mathrm{~Hz}, 2 \mathrm{H}$, p-Ph), 3.81 (m, 4H, C(4, 5)H2), 3.08 (m, 4H, C(2', 6') $\left.\mathrm{H}_{2}\right), 1.40$ (m, 2H, C(4') $\left.\mathrm{H}_{2}\right)$, $1.26\left(\mathrm{~m}, 4 \mathrm{H}, \mathrm{C}\left(3^{\prime}, 5^{\prime}\right) \mathrm{H}_{2}\right)$.

13C NMR: $\quad\left(125 \mathrm{MHz}, \mathrm{CDCl}_{3}\right)$

141.7 (d, $J=7.4 \mathrm{~Hz}$, ipso-Ph), $129.2(m-\mathrm{Ph}), 121.3(p-\mathrm{Ph}), 116.2$ (d, $J=4.6 \mathrm{~Hz}, o-$ $\mathrm{Ph}), 45.0\left(\mathrm{~d}, J=2.8 \mathrm{~Hz}, \mathrm{C}\left(2^{\prime}, 6^{\prime}\right)\right), 42.5(\mathrm{~d}, J=11.0 \mathrm{~Hz}, \mathrm{C}(4,5)), 25.5(\mathrm{~d}, J=$ $\left.4.6 \mathrm{~Hz}, \mathrm{C}\left(3^{\prime}, 5^{\prime}\right)\right), 24.3\left(\mathrm{C}\left(4^{\prime}\right)\right)$.

31P NMR: $\quad\left(162 \mathrm{MHz}, \mathrm{CDCl}_{3}\right)$

13.32 .

IR: $\quad(\mathrm{KBr})$

2950 (m), 2933 (s), 2873 (m), 2853 (m), 2836 (s), 1598 (s), 1491 (s), 1465 (m), 1452 (m), 1438 (m), 1400 (m), 1383 (s), 1342 (s), 1325 (m), 1286 (s), 1249 (s), 1219 (s), 1187 (s), 1166 (s), 1147 (m), 1121 (s), 1072 (s), 1033 (s), 997 (s), 962 
(s), $863(\mathrm{~m}), 831(\mathrm{~m}), 754(\mathrm{~s}), 737(\mathrm{~m}), 708$ (s), $691(\mathrm{~s}), 591(\mathrm{~m}), 526(\mathrm{~s})$

MS: $\quad(E I, 70 \mathrm{eV})$

$342\left(\mathrm{M}^{+}+1,22\right), 341\left(\mathrm{M}^{+}, 91\right), 258$ (58), 257 (34), 153 (23), 152 (32), 119 (36),

107 (10), 106 (100), 105 (41), 104 (30), 84 (89), 77 (28).

TLC: $\quad R_{f} 0.83$ (EtOAc)

Analysis: $\quad \mathrm{C}_{19} \mathrm{H}_{24} \mathrm{~N}_{3} \mathrm{OP}(341.39)$

Calcd.: C, 66.85\%; H, 7.09\%; N, 12.31\%; P, $9.07 \%$.

Found: C, 66.66\%; H, 7.04\%; N, 12.19\%; $\quad$ P, $8.88 \%$.

\section{1,3-Di(1-naphthyl)-2-piperidino-1,3,2-diazaphospholidine 2-Oxide (11d)}

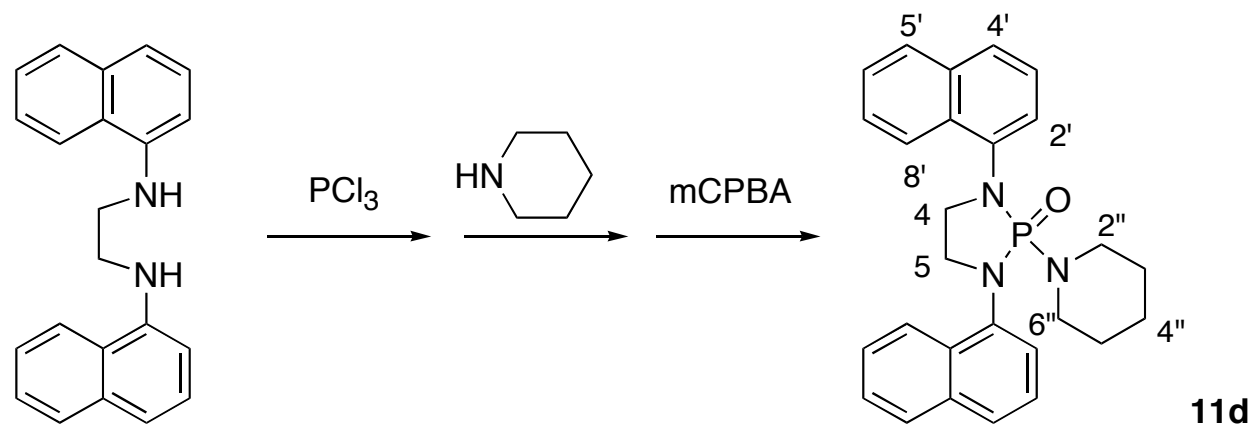

Following Representative Procedure II, to a stirred cold $\left(-78^{\circ} \mathrm{C}\right)$ solution of $\mathrm{PCl}_{3}(0.61$ $\mathrm{mL}, 7.0 \mathrm{mmol}$ ) in $10 \mathrm{~mL}$ of dry $\mathrm{CH}_{2} \mathrm{Cl}_{2}$ was added a solution of 1,2-di(1-naphthylamino)ethane ${ }^{8}$ (2.0 g, $6.4 \mathrm{mmol}$ ) in $10 \mathrm{~mL}$ of dry $\mathrm{CH}_{2} \mathrm{Cl}_{2}$ through a dropping funnel under nitrogen. Then triethylamine $(2.2 \mathrm{~mL}, 16 \mathrm{mmol})$ was added at the same temperature. The mixture was warmed to room temperature and was stirred for $24 \mathrm{~h}$. After filtration of the precipitates, the filtrate was concentrated under reduced pressure to remove the excess reagents ( $\mathrm{PCl}_{3}$ and triethylamine).

The residue was suspended in $13 \mathrm{~mL}$ of dry $\mathrm{CH}_{2} \mathrm{Cl}_{2}$ and was stirred, to which was added piperidine $(2.3 \mathrm{~mL}, 2.3 \mathrm{mmol})$ at room temperature. The mixture was stirred for $20 \mathrm{~h}$, filtered to remove the precipitates, and was concentrated under reduced pressure to remove excess piperidine.

The residue was dissolved in $10 \mathrm{~mL}$ of $\mathrm{CH}_{2} \mathrm{Cl}_{2}$ and a solution of $m$-chloroperbenzoic acid $(50 \%, 2.21 \mathrm{~g}, 6.4 \mathrm{mmol})$ in $10 \mathrm{~mL}$ of $\mathrm{CH}_{2} \mathrm{Cl}_{2}$ was added dropwise at $0{ }^{\circ} \mathrm{C}$. The mixture was allowed to warm to room temperature and was stirred for $24 \mathrm{~h}$. Then sat. aq. $\mathrm{NaHCO}_{3}$ solution 
(25 mL) was added with stirring. The aqueous layer was separated and extracted with $\mathrm{CH}_{2} \mathrm{Cl}_{2}$ (2 $\mathrm{X} 10 \mathrm{~mL}$ ). The combined organic solutions were washed with sat. aq. $\mathrm{NaHCO}_{3}$ solution, then were dried over $\mathrm{MgSO}_{4}$, filtered, and evaporated to give a residue that was chromatographed $\left(\mathrm{SiO}_{2}\right.$, benzene then benzene/ethyl acetate, 4/1). Recrystalized from diethyl ether/hexane gave $1.29 \mathrm{~g}(46 \%)$ of 11d as slightly tan crystals.

\section{Analytical Data for 11d:}

mp: $\quad 170-171^{\circ} \mathrm{C}\left(\mathrm{Et}_{2} \mathrm{O}-\right.$ hexane $)$

1

$8.44\left(\mathrm{~d}, J=8.4,2 \mathrm{H}, \mathrm{C}\left(8^{\prime}\right) \mathrm{H}\right), 7.93\left(\mathrm{~d}, J=7.3,2 \mathrm{H}, \mathrm{C}\left(2^{\prime} / 4^{\prime}\right) \mathrm{H}\right), 7.91(\mathrm{~d}, J=8.1,2 \mathrm{H}$, $\left.\mathrm{C}\left(5^{\prime}\right) \mathrm{H}\right), 7.77\left(\mathrm{~d}, J=8.2,2 \mathrm{H}, \mathrm{C}\left(2^{\prime} / 4^{\prime}\right) \mathrm{H}\right), 7.60\left(\mathrm{ddd}, J=8.4,6.8,1.3,2 \mathrm{H}, \mathrm{C}\left(7^{\prime}\right) \mathrm{H}\right)$, $7.53\left(\mathrm{ddd}, J=8.2,7.0,1.1,2 \mathrm{H}, \mathrm{C}\left(6^{\prime}\right) \mathrm{H}\right), 7.50$ (t, $\left.J=7.9,2 \mathrm{H}, \mathrm{C}\left(3^{\prime}\right) \mathrm{H}\right), 4.19$ (m, $\left.2 \mathrm{H}, \mathrm{C}(4 / 5) \mathrm{H}_{2}\right), 3.82\left(\mathrm{~m}, 2 \mathrm{H}, \mathrm{C}(4 / 5) \mathrm{H}_{2}\right), 3.06\left(\mathrm{~m}, 4 \mathrm{H}, \mathrm{C}\left(2^{\prime \prime}, 6 "\right) \mathrm{H}_{2}\right), 1.14(\mathrm{~m}, 2 \mathrm{H}$, $\left.\mathrm{C}(4 ") \mathrm{H}_{2}\right), 0.92\left(\mathrm{~m}, 4 \mathrm{H}, \mathrm{C}\left(3^{\prime \prime}, 5^{\prime \prime}\right) \mathrm{H}_{2}\right)$.

13C NMR: $\quad\left(100 \mathrm{MHz}, \mathrm{CDCl}_{3}\right)$

$138.0(\mathrm{~d}, J=4.6), 134.8,131.3$ (d, $J=5.3), 128.5,126.6,126.1,126.0,125.9$, 124.5 (d, $J=3.0), 123.5$ (br), 49.3 (d, $J=12.2$, C(4, 5)), 45.7 (d, $J=3.1, \mathrm{C}(2 "$, 6")), $26.0\left(\mathrm{~d}, J=4.6, \mathrm{C}\left(3^{\prime \prime}, 5^{\prime \prime}\right)\right), 24.1\left(\mathrm{C}\left(4^{\prime \prime}\right)\right)$.

31P NMR: $\quad\left(162 \mathrm{MHz}, \mathrm{CDCl}_{3}\right)$

16.56.

IR: $\quad(\mathrm{KBr})$

3037 (w), 3010 (w), 2964 (m), 2931 (s), 2846 (s), 1591 (s), 1574 (s), 1509 (m), 1478 (w), 1462 (s), 1441 (m), 1394 (s), 1372 (s), 1341 (s), 1276 (s), 1237 (s), 1219 (s), 1205 (s), 1180 (s), 1168 (s), 1116 (s), 1100 (s), 1080 (s), 1071 (s), 1028 (s), 1017 (m), 966 (s), 936 (m), 804 (s), 797 (s), 775 (s), 731 (s), 680 (m), 646 (m), $623(\mathrm{~m}), 605(\mathrm{w})$,

MS: $\quad(\mathrm{EI}, 70 \mathrm{eV})$ $442\left(\mathrm{M}^{+}+1,51\right), 441\left(\mathrm{M}^{+}, 100\right), 359$ (25), 358 (77), 357 (18), 291 (33), 272 (16), 203 (20), 188 (16), 186 (18), 170 (11), 169 (32), 168 (18), 167 (11), 157 (21), 156 (92), 155 (40), 154 (51), 143 (10), 130 (10), 129 (15), 128 (23), 127 (28), 115 (18), 84 (35). 
TLC: $\quad R_{f} 0.83$ (EtOAc)

Analysis: $\quad \mathrm{C}_{27} \mathrm{H}_{28} \mathrm{~N}_{3} \mathrm{OP}(441.51)$
Calcd.:
C, $73.45 \%$;
$\mathrm{H}, 6.39 \%$;
$\mathrm{N}, 9.52 \%$;
$\mathrm{P}, 7.02 \%$.
Found:
C, $73.32 \%$;
H, 6.42\%;
N, 9.61\%;
$\mathrm{P}, 6.74 \%$.

\section{1,3-Dimethyl-2-diphenylamino-1,3,2-diazaphospholidine 2-Oxide (12a)}<smiles>[M]CCN=[W]</smiles><smiles>O=[Pb][PbH2]</smiles><smiles>CN(CCN(C)P(=O)(O)N(C)C)P(N)(=O)O</smiles>

$12 a$

Following Representative Procedure I, to a solution of triethylamine $(1.74 \mathrm{~mL}, 12.5$ mmol) in $150 \mathrm{~mL}$ of dry $\mathrm{CH}_{2} \mathrm{Cl}_{2}$ were added solutions of $N, N^{\prime}$-dimethylethylenediamine $(0.53$ $\mathrm{mL}, 5.0 \mathrm{mmol})$ in $15 \mathrm{~mL}$ of dry $\mathrm{CH}_{2} \mathrm{Cl}_{2}$ and diphenylaminophosphorus dichloride $(1.43 \mathrm{~g}, 5.0$ mmol) in $15 \mathrm{~mL}$ of dry $\mathrm{CH}_{2} \mathrm{Cl}_{2}$ simultaneously and dropwise at reflux under nitrogen. The solution was heated to reflux for $15 \mathrm{~h}$ then was concentrated under reduced pressure to give a residue, which was purified by column chromatography on silica gel (ethyl acetate then ethyl acetate/2-propanol, $10 / 1$ as eluents). Recrystalization from $\mathrm{CH}_{2} \mathrm{Cl}_{2} /$ hexane afforded $1.32 \mathrm{~g}$ (87\%) of 12a as colorless needles.

Analytical Data for 12a:

mp: $\quad 115-116{ }^{\circ} \mathrm{C}\left(\mathrm{CH}_{2} \mathrm{Cl}_{2}\right.$-hexane $)$

1 H NMR: $_{\quad\left(400 \mathrm{MHz}, \mathrm{CDCl}_{3}\right)}$

$7.28(\mathrm{~m}, 8 \mathrm{H}, o, m-\mathrm{Ph}), 7.11(\mathrm{~m}, 2 \mathrm{H}, p-\mathrm{Ph}), 3.12\left(\mathrm{~m}, 2 \mathrm{H}, \mathrm{CH}_{2}\right), 2.79(\mathrm{~m}, 2 \mathrm{H}$, $\left.\mathrm{CH}_{2}\right), 2.64\left(\mathrm{~d}, J_{\mathrm{PH}}=9.5,6 \mathrm{H}, \mathrm{CH}_{3}\right)$.

13 $\mathrm{C} \mathrm{NMR:} \quad\left(100 \mathrm{MHz}, \mathrm{CDCl}_{3}\right)$

$145.2(\mathrm{~d}, J \mathrm{PC}=4.6$, ipso-Ph), $128.8(m-\mathrm{Ph}), 127.2(\mathrm{~d}, J \mathrm{PC}=3.1, o-\mathrm{Ph}), 124.9(p-$

$\mathrm{Ph}), 46.6\left(\mathrm{~d}, J_{\mathrm{PC}}=13.7, \mathrm{CH}_{2}\right), 31.2\left(\mathrm{~d}, J_{\mathrm{PC}}=6.1, \mathrm{CH}_{3}\right)$.

31P NMR: (162 MHz, $\left.\mathrm{CDCl}_{3}\right)$

19.39 . 
IR: $\quad(\mathrm{KBr})$

3090 (w), 3059 (w), 3020 (w), 3000 (w), 2987 (w), 2969 (w), 2987 (m), 2969 (m), 2934 (m), 2912 (s), 2898 (s), 2861 (s), 2820 (m), 1584 (s), 1485 (s), 1454 (s), 1444 (s), 1427 (m), 1400 (m), 1381 (s), 1355 (s), 1336 (m), 1311 (m), 1254 (s), 1203 (s), 1173 (s), 1088 (m), 1074 (s), 1040 (s), 1033 (s), 1005 (s), 981 (s), 945 (s), 925 (s), 894 (s), 862 (m), 851 (m), 762 (s), 733 (s), 721 (s), 713 (s), 696 (s), $667(\mathrm{~s}), 610(\mathrm{~m}), 604(\mathrm{~m}), 541(\mathrm{~s}), 516(\mathrm{~m})$

MS: $\quad(\mathrm{EI}, 70 \mathrm{eV})$

$302\left(\mathrm{M}^{+}+1,9\right), 301\left(\mathrm{M}^{+}, 51\right), 169$ (5), 168 (5), 167 (9), 134 (5), 133 (100), 90 (6), 69 (9).

TLC: $\quad R_{f} 0.61(\mathrm{EtOAc} / i-\mathrm{PrOH}, 9 / 1)$

Analysis: $\quad \mathrm{C}_{16} \mathrm{H}_{20} \mathrm{~N}_{3} \mathrm{OP}(301.33)$

Calcd.: C, 63.78\%; H, 6.69\%; N, 13.95\%; P, $10.28 \%$.

Found: C, 63.73\%; $\mathrm{H}, 6.79 \% ; \quad \mathrm{N}, 14.21 \% ; \quad \mathrm{P}, 9.97 \%$.

\section{1,3-Diphenyl-2-diphenylamino-1,3,2-diazaphospholidine 2-Oxide (12b)}

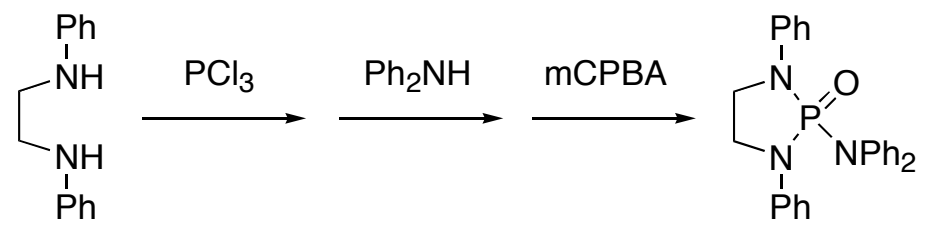

$12 b$

Following Representative Procedure II, to a stirred cold $\left(-78^{\circ} \mathrm{C}\right)$ solution of $\mathrm{PCl}_{3}(0.5$ $\mathrm{mL}, 5.73 \mathrm{mmol}$ ) in $20 \mathrm{~mL}$ of dry $\mathrm{CH}_{2} \mathrm{Cl}_{2}$ was added a solution of $N, N$ '-diphenylethylene diamide $(1.10 \mathrm{~g}, 5.17 \mathrm{mmol})$ in $5 \mathrm{~mL}$ of dry $\mathrm{CH}_{2} \mathrm{Cl}_{2}$ immediately followed by addition of triethylamine $(1.8 \mathrm{~mL}, 13 \mathrm{mmol})$. The mixture was warmed to $\mathrm{rt}$ and stirring was continued overnight. Solvent, excess $\mathrm{PCl}_{3}$ and triethylamine were removed under reduced pressure.

The residue was suspended in $10 \mathrm{~mL}$ of dry $\mathrm{CH}_{2} \mathrm{Cl}_{2}$ and a solution of diphenylamine $(0.875 \mathrm{~g}, 5.17 \mathrm{mmol})$ dissolved in triethylamine $(2.2 \mathrm{~mL}, 15.5 \mathrm{mmol})$ was added to it via a syringe, which was washed with $5 \mathrm{~mL}$ of dry $\mathrm{CH}_{2} \mathrm{Cl}_{2}$. The mixture was stirred at $\mathrm{rt}$ for $36 \mathrm{~h}$ and then was concentrated under reduced pressure to remove excess triethylamine. 
The residue was suspended in $\mathrm{CH}_{2} \mathrm{Cl}_{2}(25 \mathrm{~mL})$ and was treated with $\mathrm{mCPBA}(50 \%$, $1.78 \mathrm{~g}, 5.17 \mathrm{mmol}$ ) at $0{ }^{\circ} \mathrm{C}$. The mixture was stirred at $0{ }^{\circ} \mathrm{C}$-rt for $20 \mathrm{~h}$, then sat. aq. $\mathrm{NaHCO}_{3}$ solution $(25 \mathrm{~mL})$ was added with stirring. The aqueous layer was separated and extracted with $\mathrm{CH}_{2} \mathrm{Cl}_{2}$ (2 X $10 \mathrm{~mL}$ ). The combined organic solutions were washed with sat. aq. $\mathrm{NaHCO}_{3}$ (10 $\mathrm{mL}$ ), dried over $\mathrm{MgSO}_{4}$, filtered, and evaporated to give a crude material. The product was purified by column chromatography on silica gel $\left(\mathrm{CH}_{2} \mathrm{Cl}_{2}\right.$ /hexane, $1 / 2$ then $\mathrm{CH}_{2} \mathrm{Cl}_{2} /$ hexane/2propanol, 1/2/0.03) and recrystallization from benzene/hexane to give $1.59 \mathrm{~g}(72 \%)$ of $\mathbf{1 2 b}$ as colorless needles.

\section{Analytical Data for 12b:}

mp: $\quad 210-211^{\circ} \mathrm{C}$ (benzene/hexane)

1

$7.35(\mathrm{t}, J=7.8,4 \mathrm{H}, m-\mathrm{Ph}), 7.23(\mathrm{~d}, J=7.8,4 \mathrm{H}, o-\mathrm{Ph}), 7.21(\mathrm{t}, J=7.8,4 \mathrm{H}, m-$ $\left.\mathrm{Ph}^{\prime}\right), 7.13$ (t, $\left.J=7.3,2 \mathrm{H}, p-\mathrm{Ph}^{\prime}\right), 7.08-7.03$ (m, 6H, o-Ph', $\left.p-\mathrm{Ph}\right), 3.62(\mathrm{~m}, 2 \mathrm{H}$, $\left.\mathrm{CH}_{2}\right), 3.14\left(\mathrm{~m}, 2 \mathrm{H}, \mathrm{CH}_{2}\right)$.

13C NMR: $\quad\left(100 \mathrm{MHz}, \mathrm{CDCl}_{3}\right)$

143.6 (d, $J=5.3$, ipso-Ph), 141.1 (d, $J=7.6$, ipso-Ph), $129.3(m-\mathrm{Ph}), 129.0$ ( $m$ $\mathrm{Ph}), 127.4$ (d, $J=2.3, o-\mathrm{Ph}), 125.8$ ( $p-\mathrm{Ph}), 121.8$ ( $p-\mathrm{Ph}), 116.6(\mathrm{~d}, J=5.3, o-\mathrm{Ph})$, $42.2\left(\mathrm{~d}, J=12.2, \mathrm{CH}_{2}\right)$

31P NMR: $\quad\left(162 \mathrm{MHz}, \mathrm{CDCl}_{3}\right)$

7.43

IR: $\quad(\mathrm{KBr})$

3064 (m), 3055 (m), 3036 (m), 2970 (w), 2946 (m), 2888 (m), 2874 (m), 1597 (s), 1492 (s), 1467 (s), 1451 (m), 1401 (m), 1383 (m), 1362 (s), 1353 (s), 1310 (s), 1267 (s), 1255 (s), 1231 (s), 1213 (s), 1149 (m), 1127 (s), 1106 (s), 1077 (s), 1031 (s), 1019 (s), 996 (s), 958 (s), 935 (m), 751 (s), 701 (s), 691 (s), 586 (s), 531 (s)

MS: $\quad(\mathrm{EI}, 70 \mathrm{eV})$

$426\left(\mathrm{M}^{+}+1,44\right), 425\left(\mathrm{M}^{+}, 90\right), 258$ (38), 257 (100), 169 (10), 168 (15), 167 (22), 152 (64), 106 (16), 105 (46), 104 (37), 91 (10), 77 (38).

TLC: $\quad R f 0.90($ EtOAc) 
Analysis: $\quad \mathrm{C}_{26} \mathrm{H}_{24} \mathrm{~N}_{3} \mathrm{OP}(425.47)$

$\begin{array}{llll}\text { Calcd.: C, 73.40\%; } & \text { H, 5.69\%; } & \text { N, 9.88\%; } & \text { P, 7.28\% } \\ \text { Found: C, 73.48\%; } & \text { H, 5.63\%; } & \text { N, 9.92\%; } & \text { P, 6.98\% }\end{array}$

\section{Catalyzed Aldol Additions of Trichlorosilyl Enolates}

General Procedure for Catalyzed Aldol Additions of Trichlorosilyl Enolates 4 and (Z)-9 with Fast Addition of Aldehyde: (-)-(2R,1'S)-2-(Hydroxyphenylmethyl)cyclohexanone (anti-6a)

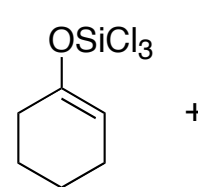

4

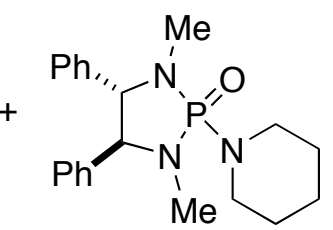

$(S, S)-7 a$

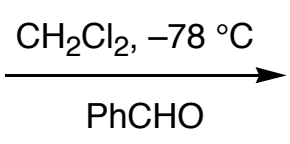<smiles>O=C1CCCC[C@H]1[C@H](O)c1ccccc1</smiles>

(-)-anti-6a

Catalyst $(S, S)-7 \mathbf{a}\left(7.3 \mathrm{mg}, 0.02 \mathrm{mmol}, 0.1\right.$ equiv) was dissolved in $\mathrm{CH}_{2} \mathrm{Cl}_{2}(2 \mathrm{~mL})$ and was cooled to $-78{ }^{\circ} \mathrm{C}$. Trichlorosilyl enolate $4 \mathbf{b}(40 \mu \mathrm{L}, 0.22 \mathrm{mmol}, 1.1$ equiv) was added dropwise over $1 \mathrm{~min}$ Benzaldehyde $(20.3 \mu \mathrm{L}, 1.0 \mathrm{mmol})$ was then added neat, over $20 \mathrm{sec}$. The reaction mixture was stirred at $-78{ }^{\circ} \mathrm{C}$ for $2 \mathrm{~h}$, then sat. aq. $\mathrm{NaHCO}_{3}$ solution $(5 \mathrm{~mL})$ was added quickly and the mixture was allowed to warm to rt. The phases were separated, and the aqueous phase was extracted with $\mathrm{CH}_{2} \mathrm{Cl}_{2}(3 \mathrm{X} 10 \mathrm{~mL})$. The organic phases were combined, dried over $\mathrm{Na}_{2} \mathrm{SO}_{4}$, filtered and concentrated in vacuo. The syn/anti ratio was determined by ${ }^{1} \mathrm{H}$ NMR (400 MHz) analysis to be $1 / 28$. The crude material was purified by column chromatography $\left(\mathrm{SiO}_{2}\right.$, hexane/EtOAc, 6/1) to give $1.3 \mathrm{mg}$ of (-)-syn-6a as an oil and $35.3 \mathrm{mg}$ (90\% total) of (-)anti-6a as a clear oil.

\section{Analytical Data for (-)-anti-6a:}

mp: $\quad 41-42^{\circ} \mathrm{C}$

1ㅁN $\underline{\text { NMR: }} \quad\left(400 \mathrm{MHz}, \mathrm{CDCl}_{3}\right)$

7.36-7.26 (m, $5 \mathrm{H}, \mathrm{Ph}), 4.78(\mathrm{dd}, J=9.2$ and 2.4, $1 \mathrm{H}, \mathrm{PhCHOH}), 3.98(\mathrm{~d}, J=2.4$, $1 \mathrm{H}, \mathrm{OH}), 2.64-2.58\left(\mathrm{~m}, 1 \mathrm{H}, \mathrm{C}(2) \mathrm{H}_{\mathrm{ax}}\right), 2.50-2.44$ (m, $\left.1 \mathrm{H}, \mathrm{C}(6) \mathrm{H}_{\mathrm{eq}}\right), 2.39-2.31$ 
(m, $\left.1 \mathrm{H}, \mathrm{C}(6) \mathrm{H}_{\mathrm{ax}}\right), 2.11-2.03$ (m, $\left.1 \mathrm{H}, \mathrm{C}(5) \mathrm{H}_{\mathrm{eq}}\right), 1.80-1.74$ (m, $\left.1 \mathrm{H}, \mathrm{C}(4) \mathrm{H}_{\mathrm{eq}}\right)$, 1.71-1.47 (m, $\left.3 \mathrm{H}, \mathrm{C}(5) \mathrm{H}_{\mathrm{ax}}, \mathrm{C}(3) \mathrm{H}_{\mathrm{eq}}, \mathrm{C}(4) \mathrm{H}_{\mathrm{ax}}\right), 1.34-1.23$ (m, $\left.1 \mathrm{H}, \mathrm{C}(3) \mathrm{H}_{\mathrm{ax}}\right)$

${ }^{13} \underline{\mathrm{C} \mathrm{NMR}}: \quad\left(100.6 \mathrm{MHz}, \mathrm{CDCl}_{3}\right)$

$215.53(\mathrm{C}=\mathrm{O}), 140.84$ (ipso-Ph), $128.30(m-\mathrm{Ph}), 127.81(p-\mathrm{Ph}), 126.95(o-\mathrm{Ph})$, $74.64(\mathrm{CHOH}), 57.35(\mathrm{C}(2)), 42.60(\mathrm{C}(6)), 30.76(\mathrm{C}(3)), 27.74(\mathrm{C}(5)), 24.63$ (C(4))

IR: $\quad\left(\mathrm{CHCl}_{3}\right)$

3536 (m, OH), 3066 (m), 3033 (m), 2945 (s), 2904 (m), 2868 (m), 1697 (s, C=O), 1497 (m), 1450 (s), 1426 (m), 1400 (m), 1322 (m), 1312 (m), 1297(m), 1226 (s), 1201(s), 1191 (m), 1130 (s, C-O), 1101 (m), 1064 (m), 1041 (s), 1029 (m), 1016 (m), $846(\mathrm{~m}), 777(\mathrm{~m}), 731(\mathrm{~m}), 724(\mathrm{~m}), 707(\mathrm{~m})$

MS: $\quad(E I, 70 \mathrm{eV})$

$204\left(\mathrm{M}^{+}, 6\right), 186\left(\mathrm{M}^{+}-\mathrm{H}_{2} \mathrm{O}, 21\right), 106\left(\mathrm{M}^{+}-\mathrm{C}_{6} \mathrm{H}_{10} \mathrm{O}, 40\right), 98\left(\mathrm{M}^{+}-\mathrm{C}_{7} \mathrm{H}_{6} \mathrm{O}\right.$, 100), 70 (48), 55 (33)

TLC: $\quad R_{f} 0.24$ (hexane/EtOAc, 3/1)

Opt. Rot.: $\quad[\alpha]_{\mathrm{D}}^{24}-24.2^{\circ}\left(c=1.03, \mathrm{CHCl}_{3}\right)$

HPLC: $\quad t_{\mathrm{R}}\left(2 R, 1^{\prime} S\right)-6 a \mathrm{a} 16.2 \mathrm{~min}(4.6 \%) ; t_{\mathrm{R}}\left(2 S, 1^{\prime} R\right)-6 \mathrm{a} 19.6 \mathrm{~min}(95.4 \%)$ (Chiracel OJ, 90/10 hexane $/ i$-PrOH, $\left.0.5 \mathrm{~mL} \mathrm{~min}^{-1}\right)$

HPLC: $\quad t_{\mathrm{R}}\left(2 S, 1^{\prime} S\right)-6 \mathrm{a} 16.8 \min (40.5 \%) ; t_{\mathrm{R}}\left(2 R, 1^{\prime} R\right)-\mathbf{6 a} 22.3 \min (59.5 \%)$ (Chiracel OJ, 90/10 hexane/i-PrOH, $0.5 \mathrm{~mL} \mathrm{~min}^{-1}$ )

Analysis: $\quad \mathrm{C}_{13} \mathrm{H}_{16} \mathrm{O}_{2}(204.27)$

Calcd.: C, $76.44 \%$; H, 7.90\%

Found: $\mathrm{C}, 76.45 \%$; $\mathrm{H}, 7.80 \%$ 


\section{General Procedure for Catalyzed Aldol Additions of 4 with Slow Addition of Aldehyde:} (-)-(2R,1'S)-2-(Hydroxyphenylmethyl)cyclohexanone (anti-6a)<smiles>COC1=CCCCC1</smiles>

4

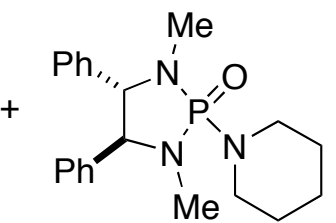

$(S, S)-7 \mathbf{a}$

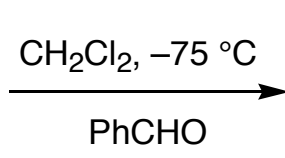

(-)-anti-6a

Catalyst $(S, S)-7 \mathbf{a}(37.6 \mathrm{mg}, 0.1 \mathrm{mmol}, 0.1$ equiv) was dried under vacuum $(0.05 \mathrm{mmHg})$ for 12 at $\mathrm{rt}, \mathrm{CH}_{2} \mathrm{Cl}_{2}(5 \mathrm{~mL})$ was added and the solution was cooled to $-75^{\circ} \mathrm{C}$ (internal). Trichlorosilyl enolate 4 (200 $\mu \mathrm{L}, 1.1 \mathrm{mmol}, 1.1$ equiv) was added dropwise over 2 min A solution of benzaldehyde $(102.0 \mu \mathrm{L}, 1.0 \mathrm{mmol})$ in $\mathrm{CH}_{2} \mathrm{Cl}_{2}(5 \mathrm{~mL})$ was then added to the first solution, dropwise, via cannula over 45 min During the addition the temperature remained at $-75^{\circ} \mathrm{C}$. The reaction mixture was stirred at $-75^{\circ} \mathrm{C}$ for $30 \mathrm{~min}$, then it was quickly poured into cold $\left(0^{\circ} \mathrm{C}\right)$ sat. aq. $\mathrm{NaHCO}_{3}$ solution $(10 \mathrm{~mL})$ and the slurry was stirred for $15 \mathrm{~min}$ The twophase mixture was filtered through Celite, the phases were separated, and the aqueous phase was extracted with $\mathrm{CH}_{2} \mathrm{Cl}_{2}(3 \times 50 \mathrm{~mL})$. The organic phases were combined, dried over $\mathrm{Na}_{2} \mathrm{SO}_{4}$, filtered and concentrated. The syn/anti ratio was determined by ${ }^{1} \mathrm{H}$ NMR (500 MHz) analysis to be $1 />50$. The crude material was purified by column chromatography $\left(\mathrm{SiO}_{2}\right.$, hexane/EtOAc, 6/1) to give $2.4 \mathrm{mg}$ of (-)-syn-6a as an oil and $189.8 \mathrm{mg}$ (94\% total) of (-)-anti-6a as a clear oil . Analytical Data for (-)-syn-6a:

SFC: $\quad t \mathrm{R}\left(2 S, 1^{\prime} S\right)-6 \mathbf{a} 2.62 \min (41.0 \%) ; t \mathrm{R}\left(2 R, 1^{\prime} R\right)-6 \mathbf{a} 3.26 \min (59.0 \%)$ (Chiralcel OJ, 150 bar, $40{ }^{\circ} \mathrm{C}, 6 \% \mathrm{CH}_{3} \mathrm{OH}$ in $\mathrm{CO}_{2}, 2.5 \mathrm{~mL} \mathrm{~min}^{-1}$ )

Analytical Data for (-)-anti-6a:

SFC: $\quad t \mathrm{R}\left(2 S, 1^{\prime} R\right)-\mathbf{6 a} 2.23 \min (4.5 \%) ; t \mathrm{R}\left(2 R, 1^{\prime} S\right)-6 \mathbf{a} 2.54 \min (95.5 \%)$ (Chiralcel OJ, 150 bar, $40{ }^{\circ} \mathrm{C}, 6 \% \mathrm{CH}_{3} \mathrm{OH}$ in $\mathrm{CO}_{2}, 2.5 \mathrm{~mL} \mathrm{~min}^{-1}$ ) 


\section{Reaction between (Z)-9 and Benzaldehyde Promoted by Phosphoramide $(S, S)-7$ a}

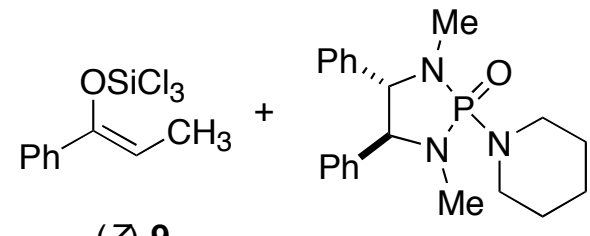

$(S, S)-7 a$

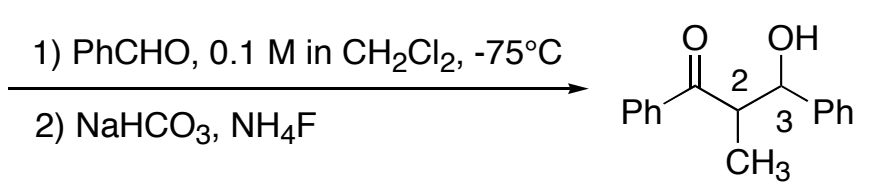

10

Phosphoramide $(S, S)-7 \mathbf{a}(34.7 \mathrm{mg}, 0.079 \mathrm{mmol})$ was dried at room temperature under high vacuum (ca. $0.05 \mathrm{mmHg}$ ) for $3 \mathrm{~h}$ in a two-necked round-bottomed flask. To this were added trichlorosilyl enolate $(Z)-9(113 \mathrm{~mL}, 0.55 \mathrm{mmol})$ and $5 \mathrm{~mL}$ of dry $\mathrm{CH}_{2} \mathrm{Cl}_{2}$ successively under nitrogen. After the solution was cooled to $-75{ }^{\circ} \mathrm{C}$ (internal temperature, dry ice-2-propanol bath), benzaldehyde $(50 \mathrm{~mL}, 0.49 \mathrm{mmol})$ was added dropwise and the mixture was stirred at $-75^{\circ} \mathrm{C}$ for $6 \mathrm{~h}$.

The reaction mixture was poured into a mixture of $5 \mathrm{~mL}$ of sat. aq. $\mathrm{NaHCO} 3$ solution and $8 \mathrm{~mL}$ of $20 \%$ aqueous $\mathrm{NH}_{4} \mathrm{~F}$ solution at $0{ }^{\circ} \mathrm{C}$ and was stirred for $1 \mathrm{~h}$ at room temperature. After separation of the organic layer, the aqueous layer was extracted with $\mathrm{CH}_{2} \mathrm{Cl}_{2}(2 \mathrm{X} 10 \mathrm{~mL})$. The combined organic extracts were dried over $\mathrm{MgSO}_{4}$, filtered, and concentrated under reduced pressure to give the crude products.

By the ${ }^{1} \mathrm{H}$ NMR analysis of of the crude products (comparison of the integrarions of $\mathrm{C}(3) H$ of $\mathbf{1 0}$; d 5.25 (br) for syn-10 and d 5.00 (dd) for anti-10), the diastereomeric ratio was determined to be syn/anti $=1 / 1.4$. The crude mixture was chromatographed on silica gel (hexane/ethyl acetate $=20 / 1.5)$ to give syn-10 $(26.6 \mathrm{mg}, 0.111 \mathrm{mmol})$ and anti-10 $(42.0 \mathrm{mg}$, $0.173 \mathrm{mmol})$. Total yield was $58 \%$.

\section{Reaction in $8 \mathrm{~min}$}

Reactions were performed as mentioned above but the reaction time was $8 \mathrm{~min}$ In the reactions of the promoters 11a, 11b, 11c, and HMPA, $0.20 \mathrm{~mL}(1.1 \mathrm{mmol})$ of 4 and $0.10 \mathrm{~mL}$ $(0.98 \mathrm{mmol})$ of benzaldehyde were used. In the reactions of 11d, 12a, and 12b, $100 \mathrm{~mL}(0.55$ mmol) of enolate 4 and $50 \mathrm{~mL}(0.49 \mathrm{mmol})$ of benzaldehyde were used.

Conversion was determined by comparison of ${ }^{1} \mathrm{H}$ NMR integrations of the aromatic 
signal of remained benzaldehyde $(\mathrm{d} 7.88(\mathrm{~d}, 2 \mathrm{H}, o-\mathrm{Ph}))$ and the $\mathrm{C}\left(1^{\prime}\right) \mathrm{H}$ signals of $\mathbf{6 a}$.

\section{Reaction for Loading Effect}

According to the general method, most of the reactions for loading effect were performed in $0.5 \mathrm{mmol} \mathrm{scale}$; i.e. using $50 \mathrm{~mL}(0.49 \mathrm{mmol})$ of benzaldehyde and $100 \mathrm{~mL}(0.55 \mathrm{mmol})$ of enolate 4 or $113 \mathrm{~mL}$ of enolate $(Z)-9$ in $5 \mathrm{~mL}$ of dry $\mathrm{CH}_{2} \mathrm{Cl}_{2}$. Other procedure was the same.

When the loading of the promoter was more than $40 \mathrm{~mol} \%$, the reaction scale was 0.25 mmol; i.e. using $25 \mathrm{~mL}(0.25 \mathrm{mmol})$ of benzaldehyde and $50 \mathrm{~mL}(0.28 \mathrm{mmol})$ of enolate 4 in 2.5 $\mathrm{mL}$ of dry $\mathrm{CH}_{2} \mathrm{Cl}_{2}$.

\section{Concentration Effect}

(1 M reaction) Phosphoramide 12a $(15.6 \mathrm{mg}, 0.052 \mathrm{mmol})$ was dried at room temperature under high vacuum (ca. $0.05 \mathrm{mmHg}$ ) for $3 \mathrm{~h}$ in a two-necked round-bottomed flask. To this were added trichlorosilyl enolate $4(0.10 \mathrm{~mL}, 0.55 \mathrm{mmol})$ and $0.5 \mathrm{~mL}$ of dry $\mathrm{CH}_{2} \mathrm{Cl}_{2}$ successively under nitrogen. After the solution was cooled to $-75^{\circ} \mathrm{C}$ (internal temperature, dry ice-2-propanol bath), benzaldehyde $(50 \mathrm{~mL}, 0.49 \mathrm{mmol})$ was added dropwise and the mixture was stirred at -75 ${ }^{\circ} \mathrm{C}$ for $1.5 \mathrm{~h}$.

(0.01 $\mathrm{M}$ reaction) Procedure was the same as above except using $50 \mathrm{~mL}$ of dry $\mathrm{CH}_{2} \mathrm{Cl}_{2}$ as the solvent and the reaction time extended to $6 \mathrm{~h}$.

Quenching procedure, determination of the product ratio, and isolation of the product were the same in both cases as those of the general method.

\section{Loading Effect of Aldehyde}

Phosphoramide 11c (18.5 mg, $0.054 \mathrm{mmol})$ was dried at room temperature under high vacuum (ca. $0.05 \mathrm{mmHg}$ ) for $3 \mathrm{~h}$ in a two-necked round-bottomed flask. To this were added trichlorosilyl enolate $4(0.10 \mathrm{~mL}, 0.55 \mathrm{mmol})$ and $5 \mathrm{~mL}$ of dry $\mathrm{CH}_{2} \mathrm{Cl}_{2}$ successively under nitrogen. After the solution was cooled to $-75^{\circ} \mathrm{C}$ (internal temperature, dry ice-2-propanol bath), benzaldehyde $(0.20 \mathrm{~mL}, 1.97 \mathrm{mmol})$ was added dropwise and the mixture was stirred at $-75^{\circ} \mathrm{C}$ for $3 \mathrm{~h}$. Quenching procedure and determination of the product ratio were the same as those of the general method. 


\section{Phosphoramide-Promoted Reaction in the Presence of Acid}

Phosphoramide 12a (3.4 mg, $0.011 \mathrm{mmol})$ was dried at room temperature under high vacuum (ca. $0.05 \mathrm{mmHg}$ ) for $3 \mathrm{~h}$ in a two-necked round-bottomed flask. To this were added trichlorosilyl enolate $4(100 \mathrm{~mL}, 0.55 \mathrm{mmol})$ and $4 \mathrm{~mL}$ of dry $\mathrm{CH}_{2} \mathrm{Cl}_{2}$ successively under nitrogen. After the solution was cooled to $-75^{\circ} \mathrm{C}$ (dry ice-2-propanol bath), benzoic acid (1.4 $\mathrm{mg}, 0.011 \mathrm{mmol})$ in $1 \mathrm{~mL}$ of dry $\mathrm{CH}_{2} \mathrm{Cl}_{2}$ and benzaldehyde $(50 \mathrm{~mL}, 0.49 \mathrm{mmol})$ were added successively and the mixture was stirred at $-75^{\circ} \mathrm{C}$ for $3 \mathrm{~h}$. The cold reaction mixture was poured into a mixture of $5 \mathrm{~mL}$ of saturated aqueous $\mathrm{NaHCO}_{3}$ and $8 \mathrm{~mL}$ of $20 \%$ aqueous $\mathrm{NH}_{4} \mathrm{~F}$ at $0{ }^{\circ} \mathrm{C}$ with vigorous stirring, which was continued for $30 \mathrm{~min}$ at $\mathrm{rt}$. Then insoluble material was filtered through a celite pad and the aqueous layer was extracted with $\mathrm{CH}_{2} \mathrm{Cl}_{2}$ twice. The combined organic solutions were dried ( $\left.\mathrm{MgSO}_{4}\right)$ and condensed to give white solid.

The product ratio was determined by ${ }^{1} \mathrm{H}$ NMR as metioned above. The product $6 \mathbf{a}$ was isolated ( $86.8 \mathrm{mg}, 87 \%)$ by column chromatography.

Other experiments in Tables 13 and 14 were performed in a similar way. For triflic acid, a $\mathrm{CH}_{2} \mathrm{Cl}_{2}$ solution $(0.113 \mathrm{M})$ was prepared in advance by diluting $20 \mathrm{~mL}(0.226 \mathrm{mmol})$ of triflic acid with $2 \mathrm{~mL}$ of dry $\mathrm{CH}_{2} \mathrm{Cl}_{2}$ under nitrogen.

\section{Aldol Addition Reactions of Trichlorosilyl Enolate 4 to Benzaldehyde Catalyzed by $(S, S)-7$ a}

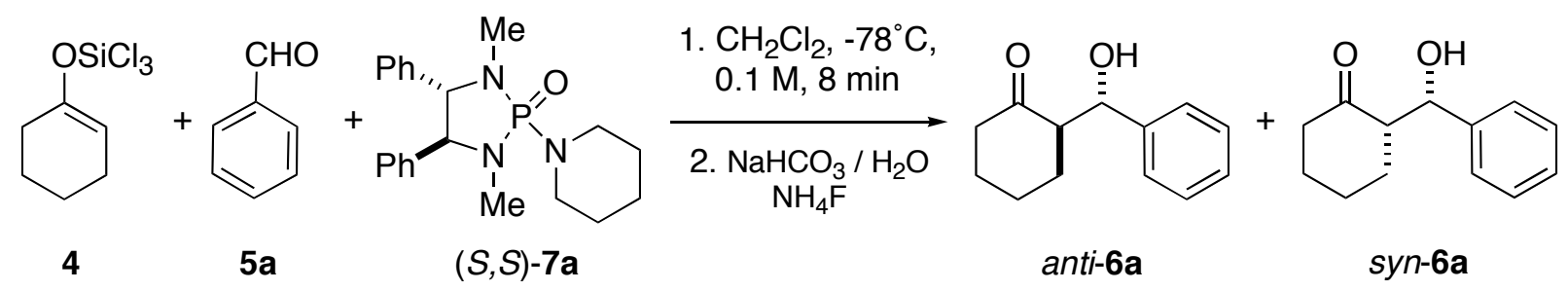

The phosphoramide $(S, S)$-7a $(36.9 \mathrm{mg}, 0.1 \mathrm{mmol}, 0.1$ equiv) was weighed into a dry flask and dried under high vacuum for $4 \mathrm{~h}$ and then $\mathrm{CH}_{2} \mathrm{Cl}_{2}(10 \mathrm{~mL})$ was added under argon. The solution was then cooled to $-78^{\circ} \mathrm{C}$ and trichlorosilyl enolate 4 (201 $\mu \mathrm{L}, 1.1 \mathrm{mmol}, 1.1$ equiv) was added neat and the solution stirred for $10 \mathrm{~min}$ Benzaldehyde (167 $\mu \mathrm{L}, 1.0 \mathrm{mmol}, 1.0$ equiv) was added into the reaction mixture via a syringe along the wall of the flask and the reaction mixture was stirred at $-78{ }^{\circ} \mathrm{C}$ for $8 \mathrm{~min}$ and then poured into a mixture of sat. aq. $\mathrm{NaHCO}_{3}$ (10 
$\mathrm{mL})$ solution and $20 \%$ aq. $\mathrm{NH}_{4} \mathrm{~F}(10 \mathrm{~mL})$ solution at $0{ }^{\circ} \mathrm{C}$ with vigorous stirring. The mixture was then allowed to stir at $\mathrm{rt}$ for $30 \mathrm{~min}$ Layers were separated and the aqueous layer was extracted with $\mathrm{CH}_{2} \mathrm{Cl}_{2}(2 \times 10 \mathrm{~mL})$. The organic extracts were washed (brine), dried (Na2SO 4$)$ and concentrated in vacuo to give an oil. ${ }^{1}$ H NMR analysis showed a syn/anti ratio of $1 / 35$. The crude product was then separated by column chromatography $\left(\mathrm{SiO}_{2}\right.$, hexane/EtOAc $9 / 1$ to 3/1) to give $6.3 \mathrm{mg}$ (3\%) syn-6a, and $196.1 \mathrm{mg}$ of anti-6a (96\%) (total yield 99\%). Analysis of the products by chiral HPLC revealed syn er of 1.2/1 and anti er of 21/1.

Analytical Data for anti-6a:

HPLC: $\quad \mathrm{t}_{R}\left(\left(2 S, 1^{\prime} R\right)-\mathbf{6 a}\right) 14.36 \mathrm{~min}(4.5 \%)$, and $\mathrm{t}_{R}\left(\left(2 R, 1^{\prime} S\right)-\mathbf{6 a}\right) 17.52 \mathrm{~min}(95.5 \%)$

(Daicel ChiralCel OJ, hexane/i-PrOH, 90/10, $0.8 \mathrm{~mL} / \mathrm{min})(\mathrm{er} 21 / 1)$

Analytical Data for syn-6a

HPLC: $\quad \mathrm{t}_{R}\left(2 R, 1^{\prime} R-\mathbf{6 a}\right) 15.22 \mathrm{~min}(45.4 \%)$, and $\mathrm{t}_{R}\left(2 S, 1^{\prime} S-6 \mathbf{6 a}\right) 20.82 \mathrm{~min}(54.6 \%)$ (Daicel ChiralCel OJ, hexane/i-PrOH, 90/10, $0.8 \mathrm{~mL} / \mathrm{min})(\mathrm{er} 1.2 / 1)$

Aldol Addition Reactions of Trichlorosilyl Enolate 4 to Benzaldehyde Catalyzed by $(S, S)$ 7a (One Equivalent)

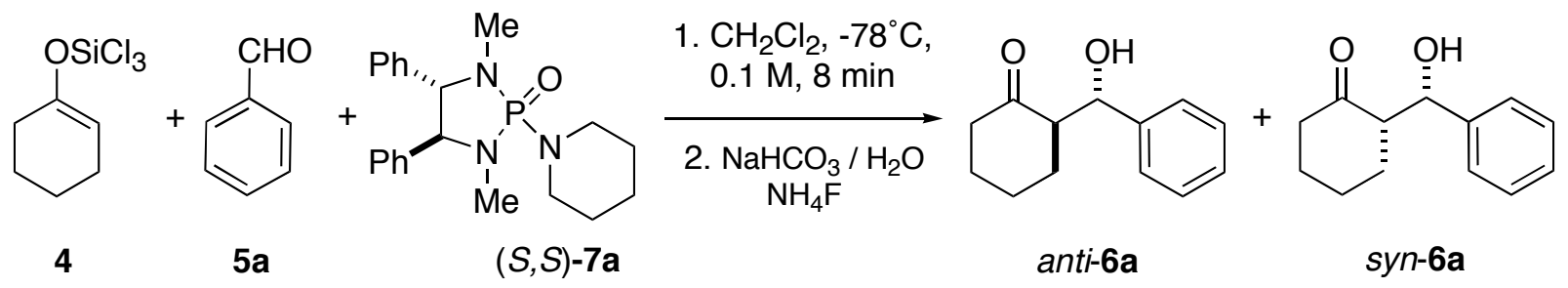

Following the procedure described above from the phosphormide $(S, S)-7 \mathbf{a}(369.3 \mathrm{mg}, 1.0$ mmol, 1.0 equiv), the trichlorosilyl enolate 4 (201 $\mu \mathrm{L}, 1.1 \mathrm{mmol}, 1.1$ equiv) and benzaldehyde (101.6 $\mu \mathrm{L}, 1.0 \mathrm{mmol}, 1.0$ equiv) in $\mathrm{CH}_{2} \mathrm{Cl}_{2}(10 \mathrm{~mL})$ was obtained $2.1 \mathrm{mg}(1 \%)$ syn-6a, and $199.1 \mathrm{mg}$ of anti-6a (97\%) (total yield 98\%). Diastereomeric ratio was determined on the crude product mixture by ${ }^{1} \mathrm{H}$ NMR analysis (syn/anti, 1/95). Analytical Data for anti-6a:

HPLC: $\quad \mathrm{t}_{R}\left(\left(2 S, 1^{\prime} R\right)-\mathbf{6 a}\right) 13.33 \min (4.15 \%)$, and $\mathrm{t}_{R}\left(\left(2 R, 1^{\prime} S\right)-6 \mathbf{6}\right) 16.28 \min (95.85 \%)$ (Daicel ChiralCel OJ, hexane/i-PrOH, 90/10, $0.8 \mathrm{~mL} / \mathrm{min})($ er 23/1) 


\section{Analytical Data for syn-6a}

HPLC: $\quad \mathrm{t}_{R}\left(2 R, 1^{\prime} R-6 \mathbf{6 a}\right) 13.96 \mathrm{~min}(15.3 \%)$, and $\mathrm{t}_{R}\left(2 S, 1^{\prime} S-6 a\right) 18.10 \mathrm{~min}(84.7 \%)$ (Daicel ChiralCel OJ, hexane $/ i-\mathrm{PrOH}, 90 / 10,0.8 \mathrm{~mL} / \mathrm{min})(\mathrm{er} 5.5 / 1)$

Nonlinear Effect Studies: Phosphoramide (S,S)-7a.
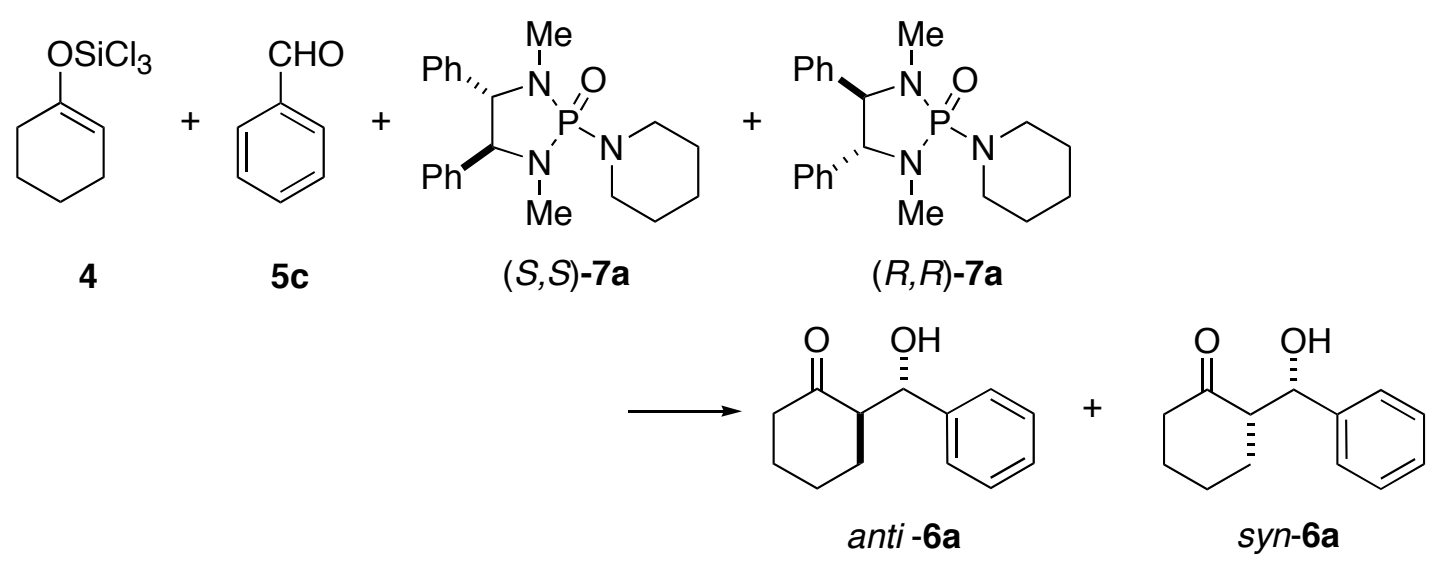

Phosphoramide $(S, S)-7 \mathbf{a}(25.89 \mathrm{mg}, 0.07008 \mathrm{mmol})$ and $(R, R)-7 \mathbf{a}(11.11 \mathrm{mg}, 0.03007$ mmol) was weighed into a round bottomed flask and dried under high vacuums for $4 \mathrm{~h}$. The phosphoramide (39.95\% e.e. based on the weights) was then dissolved in $\mathrm{CH}_{2} \mathrm{Cl}_{2}(10 \mathrm{~mL})$ under argon and the solution cooled to $-78{ }^{\circ} \mathrm{C}$. The trichlorosilyl enolate $4(201 \mu \mathrm{L}, 1.1 \mathrm{mmol})$ was then added via a syringe and solution stirred for 5 min Benzaldehyde $(102 \mu \mathrm{L}, 1.0 \mathrm{mmol})$ was then added and the solution stirred at $-78^{\circ} \mathrm{C}$ for $8 \mathrm{~min}$ The reaction mixture was then poured into a mixture of sat. aq. $\mathrm{NaHCO}_{3}$ solution and $20 \%$ aq. $\mathrm{NH}_{4} \mathrm{~F}$ solution at $0^{\circ} \mathrm{C}$ with vigorous stirring. The mixture was then stirred at $\mathrm{rt}$ for $30 \mathrm{~min}$ The layers were separated and the aqueous layer extracted with $\mathrm{CH}_{2} \mathrm{Cl}_{2}(2 \mathrm{X} 20 \mathrm{~mL})$. The combined organic solution was washed with brine, dried ( $\left.\mathrm{MgSO}_{4}\right)$ and concentrated to give oily residue, which was then purified by column chromatography ( $\mathrm{SiO}_{2}$, hexane/EtOAc, 9/1 to 3/1) to give anti-6a (189 mg, 93\%) along with syn-6a (9.2 mg, 5\%).

Analytical Data for anti-6a

HPLC: $\quad \mathrm{t}_{R}\left(\left(2 S, 1^{\prime} R\right)-6 \mathbf{a}\right) 13.65 \min (23.4 \%)$, and $\mathrm{t}_{R}\left(\left(2 R, 1^{\prime} S\right)-\mathbf{6 a}\right) 16.87 \mathrm{~min}(76.6 \%)$

(Daicel ChiralCel OJ, hexane $/ i-\mathrm{PrOH}, 90 / 10,0.8 \mathrm{~mL} / \mathrm{min})(53.3 \%$ e.e.) 
Nonlinear Effect Studies: Phosphoramide (S,S)-7j.

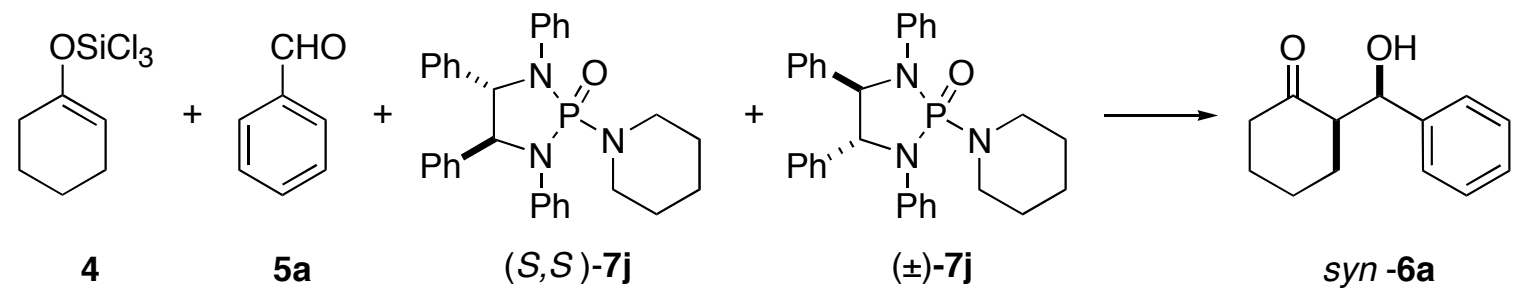

A mixture of $(S, S)-7 \mathbf{j}(4.96 \mathrm{mg}, 0.01005 \mathrm{mmol})$ and $( \pm)-7 \mathbf{j}(19.69 \mathrm{mg}, 0.03989 \mathrm{mmol})$ was dried under high vacuums over night and then dissolved in $\mathrm{CH}_{2} \mathrm{Cl}_{2}(5 \mathrm{~mL})$ under argon ( $20.1 \%$ e.e. of phosphoramide $7 \mathbf{j}$ based on weights). The solution was cooled to $-78^{\circ} \mathrm{C}$ and enolate $4(100.3 \mu \mathrm{L}, 0.55 \mathrm{mmol})$ was added. After the solution was stirred for $5 \mathrm{~min}$ at $-78^{\circ} \mathrm{C}$ benzaldehyde $(50.8 \mu \mathrm{L}, 0.50 \mathrm{mmol})$ was added and the solution was stirred at $-78^{\circ} \mathrm{C}$ for $2 \mathrm{~h}$. The reaction was then quenched by pouring into a mixture of sat. aq. $\mathrm{NaHCO}_{3}$ solution $(6 \mathrm{~mL})$ and $20 \%$ aq. $\mathrm{NH} 4 \mathrm{~F}$ solution $(6 \mathrm{~mL})$ solution at $0^{\circ} \mathrm{C}$ with vigorous stirring. The mixture was then stirred at $\mathrm{rt}$ for $1 \mathrm{~h}$ and then filtered through a layer of Celite. The layers of the filtrate were separated and the aqueous layer extracted with $\mathrm{CH}_{2} \mathrm{Cl}_{2}(2 \mathrm{X} 10 \mathrm{~mL})$. The combined organic solution was washed with brine, dried $\left(\mathrm{MgSO}_{4}\right)$ and concentrated to give solid residue (syn/anti $>100 / 1)$, which was then purified by column chromatography $\left(\mathrm{SiO}_{2}\right.$, hexane/EtOAc, 9/1) to give syn-6a (86.7 mg, 85\%). HPLC analysis showed $11.3 \%$ e.e. for syn-6a.

Analytical Data for syn-6a

HPLC: $\left.\quad \mathrm{t}_{R}\left(2 R, 1^{\prime} R\right)-\mathbf{6 a}\right) 13.35 \min (55.65 \%)$, and $\mathrm{t}_{R}\left(\left(2 S, 1^{\prime} S\right)-\mathbf{6 a}\right) 17.87 \min (44.35 \%)$ (Daicel ChiralCel OJ, hexane $/ i$-PrOH, 90/10, $0.8 \mathrm{~mL} / \mathrm{min}$ ) 


\section{Benzoic Acid Effects on the Phosphoramide ( $S, S$,)-7a Catalyzed Reactions}

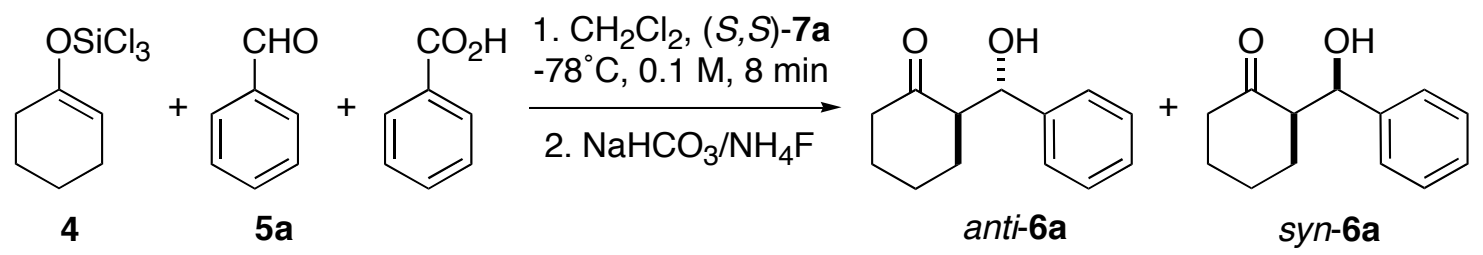

To a solution of the phosphoramide $(S, S)-,7 \mathbf{a}(36.9 \mathrm{mg}, 0.1 \mathrm{mmol}, 0.1$ equiv) and benzoic acid (12.2 mg, $0.1 \mathrm{mmol}, 0.1$ equiv) in $\mathrm{CH}_{2} \mathrm{Cl}_{2}(10 \mathrm{~mL})$ was added 4 (201 $\mu \mathrm{L}, 1.1 \mathrm{mmol}, 1.1$ equiv) via a syringe at $-78{ }^{\circ} \mathrm{C}$. The solution was stirred for 5 min before benzaldehyde $(102 \mu \mathrm{L}$, $1.0 \mathrm{mmol}, 1.0$ equiv) was added along the side wall of the flask at $-78{ }^{\circ} \mathrm{C}$. The reaction mixture was then stirred for $8 \mathrm{~min}$ at this temperature and poured into a mixture of sat. aq. $\mathrm{NaHCO}_{3}$ solution $(10 \mathrm{~mL})$ and $20 \%$ aq. $\mathrm{NH} 4 \mathrm{~F}$ solution $(10 \mathrm{~mL})$ at $0{ }^{\circ} \mathrm{C}$ with rapid stirring. The mixture was then warmed to $\mathrm{rt}$ and stirred for $30 \mathrm{~min}$ The layers were separated and aqueous layer was extracted with $\mathrm{CH}_{2} \mathrm{Cl}_{2}(2 \mathrm{X} 10 \mathrm{~mL})$. The combined extracts were washed with brine $(10 \mathrm{~mL})$ and dried (MgSO4). Solvent was removed in vacuo to give the crude product as an oil. ${ }^{1} \mathrm{H}$ NMR analysis showed conversion of $24 \%$ with syn/anti ratio of $4.1 / 1$. The crude product was then separated by column chromatography ( $\mathrm{SiO}_{2}$, hexane/EtOAc $9 / 1$ to 3/1) to give the syn product syn-6a $(28.7 \mathrm{mg}, 1.0 / 1 \mathrm{er})$ as white crystals, anti product anti-6a $(6.5 \mathrm{mg}$, er, 8.1/1) as an oil and $1.4 \mathrm{mg}$ of mixed product (total yield $18 \%$ ).

\section{Analytical Data for syn-6a}

HPLC: $\quad \mathrm{t}_{R}\left(\left(2 R, 1^{\prime} R\right)-\mathbf{1 3 8}\right) 13.92 \min (51.0 \%)$, and $\mathrm{t}_{R}\left(\left(2 S, 1^{\prime} S\right)-\mathbf{1 3 8}\right) 18.50 \min (49.0 \%)$ (Daicel ChiralCel OJ, hexane $/ i$-PrOH, 90/10, $0.8 \mathrm{~mL} / \mathrm{min}$ )

\section{Analytical Data for anti-6a}

HPLC: $\quad \mathrm{t}_{R}\left(\left(2 S, 1^{\prime} R\right)-\mathbf{6 a}\right) 13.50 \mathrm{~min}(10.8 \%)$, and $\mathrm{t}_{R}((2 R, 1 ' S)-6 \mathbf{a}) 16.34 \mathrm{~min}(89.2 \%)$ (Daicel ChiralCel OJ, hexane/i-PrOH, 90/10, $0.8 \mathrm{~mL} / \mathrm{min}$ ) 


\section{Kinetics: General Procedures.}

General Procedure 1a, GC Kinetics using zeroeth order aldehyde:

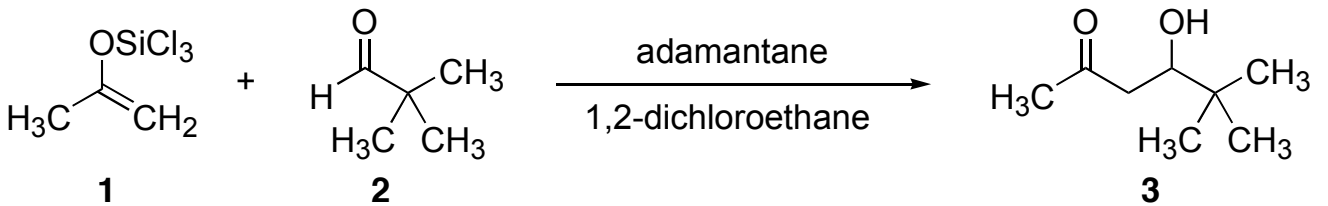

Pivalaldehyde (2) (1.16 mL, $10.4 \mathrm{mmol}, 10$ equiv.) was added rapidly to a solution of enolate, $1(150 \mu \mathrm{L}, 1.04 \mathrm{mmol})$ and adamantane $(22.3 \mathrm{mg}, 0.16 \mathrm{mmol})$ in 1,2-dichloroethane (8 $\mathrm{mL})$ at room temperature. Small aliquots were removed via cannula directly into a cold $\left(0^{\circ} \mathrm{C}\right)$ solution of concentrated KF and 1.0 M phosphate buffer (1/1, v/v). The mixture was vigorously vortexed and then was extracted with EtOAc. The mixture was passed through Florisil (plug) and quickly eluted with EtOAc. The sample was then analyzed by GC.

\section{General Procedure 1b, GC Kinetics using zeroeth order enolate:}

Pivalaldehyde (2) (29 $\mu \mathrm{L}, 0.26 \mathrm{mmol})$ was added rapidly to a solution of enolate, 1 (380 $\mu \mathrm{L}, 2.6 \mathrm{mmol}, 10$ equiv.) and adamantane $(6.8 \mathrm{mg}, 0.05 \mathrm{mmol})$ in dichloroethane $(2 \mathrm{~mL})$ at room temperature. Small aliquots were removed via cannula directly into a cold $\left(0^{\circ} \mathrm{C}\right)$ solution of concentrated KF and 1.0 M phosphate buffer $(1 / 1, \mathrm{v} / \mathrm{v})$. The mixture was vigorously vortexed and then was extracted with EtOAc. The mixture was passed through Florisil (plug) and quickly eluted with EtOAc. The sample was then analyzed by GC.

GC:

Instrument: Hewlett-Packard 5890, Hewlett-Packard 3396 Series III Integrator Conditions: Hewlett-Packard HP-5, $50 \mathrm{~m} 150{ }^{\circ} \mathrm{C}, 15 \mathrm{psi}, 4 \mu \mathrm{L}$.

Retention Times $\left(t_{R}\right): \mathbf{3}=5.8 \mathrm{~min}$; Adamantane $=7.0 \mathrm{~min}$ 
General Procedure 2a, Uncatalyzed ReactIR Kinetics:

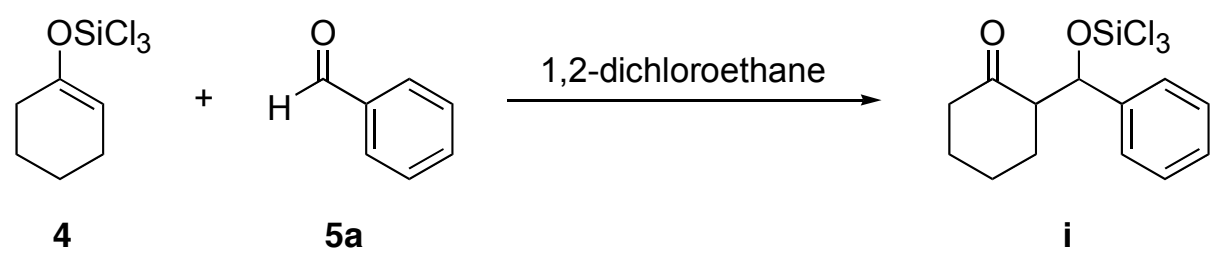

A dry reaction vessel was assembled onto the IR probe and vented for several minutes with dry nitrogen. 1,2-dichloroethane $(1.5 \mathrm{~mL})$ was then added to the reaction vessel and a background file was collected. Enolate $1(82 \mu \mathrm{L}, 0.45 \mathrm{mmol})$ was then added and allowed to fully mix and the solution was left to equilibrate before beginning data collection. Benzaldehyde (46 $\mu \mathrm{L}, 0.45 \mathrm{mmol}$ ) was added quickly to the enolate solution. IR spectra were collected at 3 min intervals for $180 \mathrm{~min}$ at $30{ }^{\circ} \mathrm{C}$ by internal monitoring. Recorded disappearance of peak at $1679 \mathrm{~cm}^{-1}$ (enolate $\left.\mathrm{C}=\mathrm{C}\right)$.

\section{General Procedure 2b, Catalyzed ReactIR Kinetics:}

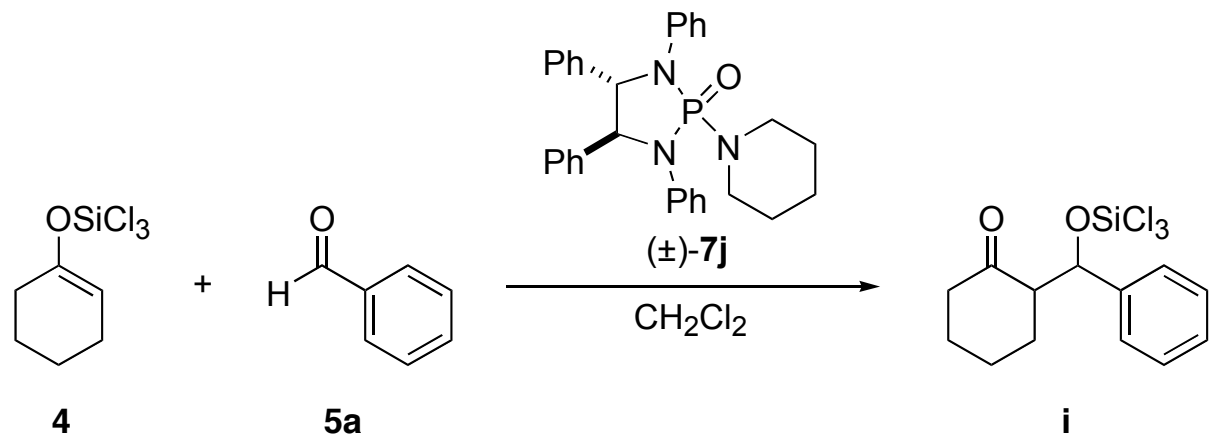

$15 \mathrm{mg}$ of ( \pm )-7j was added to a dry reaction vessel. The vessel was assembled onto the IR probe and vented for several minutes with dry nitrogen. Dry methylene chloride $(2.3 \mathrm{~mL})$ was then added to the reaction vessel. The vessel was then submerged into a chilled isopropanol bath and the contents were allowed to equilibrate prior to collecting a background file. Enolate $\mathbf{4}(111 \mu \mathrm{L}, 0.61 \mathrm{mmol})$ was then added and allowed to fully mix. Benzaldehyde $(62 \mu \mathrm{L}, 0.61$ mmol) was added quickly to the reaction solution. IR spectra were collected at $30 \mathrm{~s}$ intervals for $240 \mathrm{~min}$ at $-49^{\circ} \mathrm{C}$ by internal monitoring. Recorded disappearance of peak at $1679 \mathrm{~cm}^{-1}$ (enolate $\mathrm{C}=\mathrm{C})$.

IR:

Instrument: $\quad$ ASI ReactIR 1000

Probe and Detector: $\quad$ 5/8” DiComp Probe using an MCT detector operating at $-195{ }^{\circ} \mathrm{C}$ 
General Procedure 3a, Rapid Injection NMR Kinetics Using ( \pm )-7j:

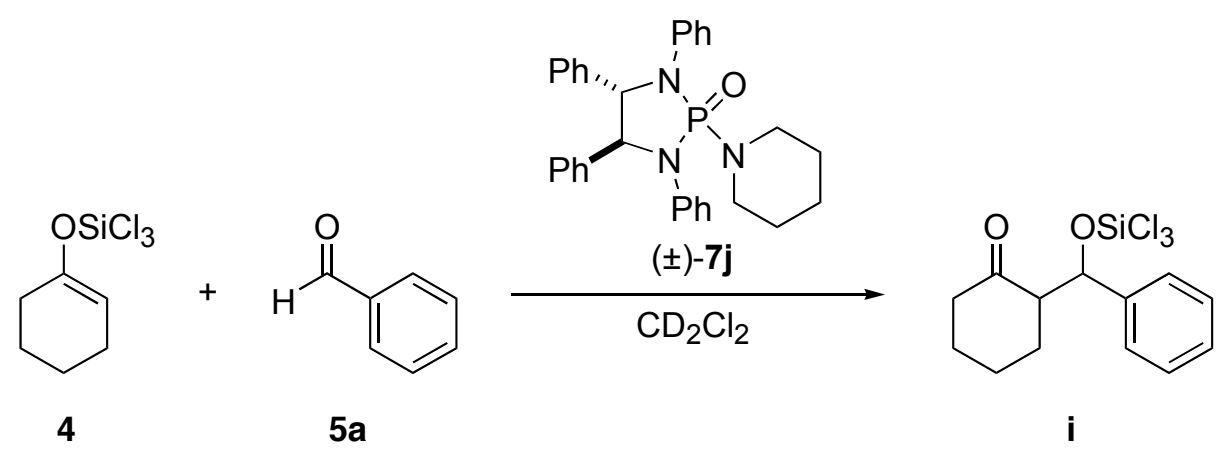

To a dry NMR tube was added phosphoramide $( \pm)-7 \mathbf{j}$ (8.6 mg, $0.017 \mathrm{mmol})$ of, $\mathrm{CD}_{2} \mathrm{Cl}_{2}$ $(600 \mu \mathrm{L})$ and enolate $4(65 \mathrm{~mL}, 0.35 \mathrm{mmol})$. The tube was vigorously mixed using a vortex shaker and stored in a short dewar at $-78^{\circ} \mathrm{C}$. Following proper positioning into spinner, the cap was carefully removed and the tube was quickly lowered into a precooled spectrometer under dry nitrogen. The fully primed RINMR injection apparatus was then carefully lowered into the spinning tube and the entire setup was allowed to equilibrate for several minutes. After equilibration, a benzaldehyde solution ( $85 \mu \mathrm{L}, 0.35 \mathrm{mmol}$ ) was then rapidly injected.

\section{General Procedure 3b, Rapid Injection NMR Kinetics Using (S,S)-7a:}

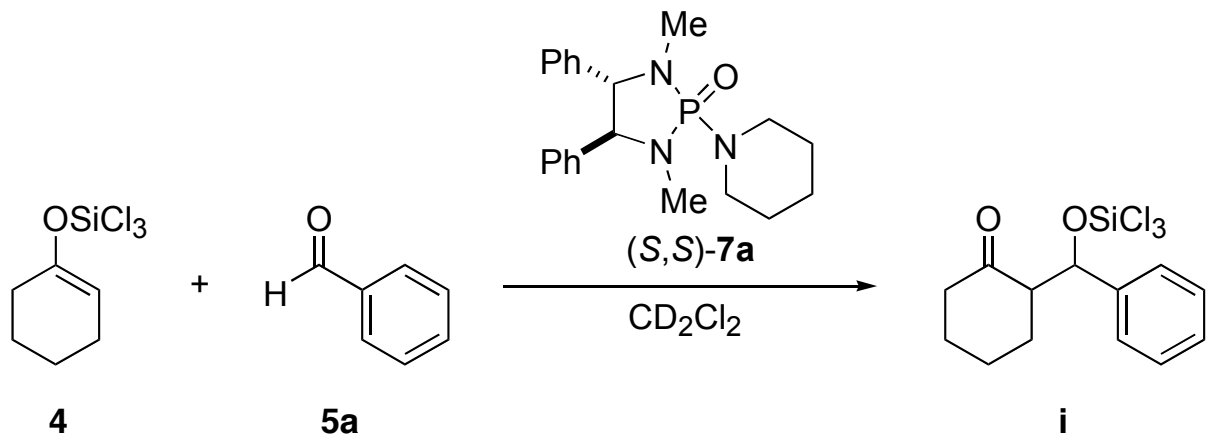

To a dry NMR tube was added $3.2 \mathrm{mg}(0.009 \mathrm{mmol})$ of phosphoramide, $600 \mu \mathrm{L}$ of $\mathrm{CD}_{2} \mathrm{Cl}_{2}, 50 \mu \mathrm{L}$ of a stock solution of $1 \% \mathrm{TMS}$ in $\mathrm{CDCl}_{3}$ followed by $16 \mu \mathrm{L}(0.086 \mathrm{mmol})$ of enolate $(S, S)-7 \mathbf{a}$. The tube was vigorously mixed using a vortex shaker and stored in a short dewar at $-78{ }^{\circ} \mathrm{C}$. Following proper positioning into spinner, the cap was carefully removed and the tube was quickly lowered into a precooled spectrometer under dry nitrogen. The fully primed RINMR injection apparatus was then carefully lowered into the spinning tube and the entire setup was allowed to equilibrate for several minutes. After equilibration a benzaldehyde solution (42 $\mu \mathrm{L}, 0.086 \mathrm{mmol})$ was then rapidly injected. 


\section{Raw Data. GC Kinetics of Addition of 1 to 2.}

Temperature: $20^{\circ} \mathrm{C}$, Run \#1:

Using General Procedure 1, pivalaldehyde (380 $\mu \mathrm{L}, 2.6 \mathrm{mmol})$ was added to a solution of enolate $1(29 \mu \mathrm{L}, 0.26 \mathrm{mmol})$ and adamantane $(6.9 \mathrm{mg}, 0.051 \mathrm{mmol})$ in 1,2-dichloroethane (2.0 $\mathrm{mL}$ ) at $20{ }^{\circ} \mathrm{C}$ as internally monitored. The reaction was monitored every 5 minutes for 90 minutes, and then incrementally thereafter until complete conversion. $\mathrm{k}_{\mathrm{obs}}=0.014 \mathrm{~min}^{-1}$.

\begin{tabular}{|cccccc|}
\hline $\begin{array}{c}\text { Time } \\
\text { (min) }\end{array}$ & $\begin{array}{c}\text { Aldolate } \\
\text { Area }\end{array}$ & $\begin{array}{c}\text { Adamantane } \\
\text { Area }\end{array}$ & Conversion & [Enolate] & In([Enolate]/[Enolate] $]_{\mathbf{0}}$ \\
\hline \hline 5 & 1441 & 5925 & 0.10 & 0.10 & 0.09 \\
10 & 1854 & 4396 & 0.16 & 0.10 & 0.15 \\
15 & 2660 & 4334 & 0.22 & 0.09 & 0.23 \\
20 & 3339 & 4400 & 0.27 & 0.08 & 0.30 \\
25 & 3644 & 4073 & 0.32 & 0.08 & 0.36 \\
30 & 4609 & 4334 & 0.38 & 0.07 & 0.45 \\
37 & 3023 & 2398 & 0.44 & 0.06 & 0.56 \\
40 & 5023 & 3583 & 0.49 & 0.06 & 0.65 \\
45 & 5957 & 4114 & 0.51 & 0.06 & 0.68 \\
50 & 5320 & 3400 & 0.55 & 0.05 & 0.76 \\
55 & 7074 & 4210 & 0.59 & 0.05 & 0.85 \\
60 & 8078 & 4575 & 0.61 & 0.04 & 0.92 \\
\hline
\end{tabular}

Temperature: $20^{\circ} \mathrm{C}$, Run \#2:

Using General Procedure 1, pivalaldehyde $(380 \mu \mathrm{L}, 2.6 \mathrm{mmol})$ was added to a solution of enolate $1(29 \mu \mathrm{L}, 0.26 \mathrm{mmol})$ and adamantane $(7.5 \mathrm{mg}, 0.055 \mathrm{mmol})$ in 1,2-dichloroethane (2.0 $\mathrm{mL})$ at $20{ }^{\circ} \mathrm{C}$ as internally monitored. The reaction was monitored every 5 minutes for 90 minutes, and then incrementally thereafter until complete conversion. $\mathrm{k}_{\mathrm{obs}}=0.015 \mathrm{~min}^{-1}$.

\begin{tabular}{|cccccc|}
\hline $\begin{array}{c}\text { Time } \\
\text { (min) }\end{array}$ & $\begin{array}{c}\text { Aldolate } \\
\text { Area }\end{array}$ & $\begin{array}{c}\text { Adamantane } \\
\text { Area }\end{array}$ & Conversion & [Enolate] & In([Enolate]/[Enolate] $]_{\mathbf{0}}$ \\
\hline \hline 5 & 0 & 0 & - & - & - \\
10 & 1446 & 3637 & 0.15 & 0.09 & 0.16 \\
15 & 2360 & 4030 & 0.21 & 0.09 & 0.24 \\
20 & 3124 & 4234 & 0.27 & 0.08 & 0.32 \\
25 & 3808 & 4184 & 0.32 & 0.07 & 0.41 \\
30 & 3966 & 3980 & 0.35 & 0.07 & 0.46 \\
35 & 5387 & 4594 & 0.41 & 0.06 & 0.57 \\
40 & 4413 & 3348 & 0.46 & 0.06 & 0.67 \\
45 & 4436 & 3162 & 0.49 & 0.05 & 0.73 \\
50 & 6356 & 4226 & 0.53 & 0.05 & 0.81 \\
55 & 6257 & 3944 & 0.55 & 0.05 & 0.88 \\
60 & 5901 & 3527 & 0.58 & 0.04 & 0.96 \\
\hline
\end{tabular}




\section{Temperature: $20^{\circ} \mathrm{C}$, Run \#3:}

Using General Procedure 1, pivalaldehyde $(380 \mu \mathrm{L}, 2.6 \mathrm{mmol})$ was added to a solution of enolate $1(29 \mu \mathrm{L}, 0.26 \mathrm{mmol})$ and adamantane $(6.9 \mathrm{mg}, 0.051 \mathrm{mmol})$ in 1,2-dichloroethane (2.0 $\mathrm{mL}$ ) at $20^{\circ} \mathrm{C}$ as internally monitored. The reaction was monitored every 5 minutes for 90 minutes, and then incrementally thereafter until complete conversion. $\mathrm{k}_{\mathrm{obs}}=0.022 \mathrm{~min}^{-1}$.

\begin{tabular}{|c|c|c|c|c|c|}
\hline $\begin{array}{l}\text { Time } \\
(\min )\end{array}$ & $\begin{array}{c}\text { Aldolate } \\
\text { Area }\end{array}$ & $\begin{array}{c}\text { Adamantane } \\
\text { Area }\end{array}$ & Conversion & [Enolate] & $\ln \left([\right.$ Enolate $\left.] /[\text { Enolate }]_{0}\right)$ \\
\hline$\overline{5}$ & 1433 & 2876 & 0.18 & 0.10 & 0.14 \\
\hline 10 & 2732 & 2914 & 0.33 & 0.08 & 0.29 \\
\hline 15 & 4211 & 3102 & 0.48 & 0.07 & 0.45 \\
\hline 20 & 4417 & 2625 & 0.59 & 0.06 & 0.59 \\
\hline 25 & 5700 & 2897 & 0.68 & 0.05 & 0.74 \\
\hline 30 & 6000 & 2731 & 0.76 & 0.05 & 0.88 \\
\hline
\end{tabular}

Temperature: $30{ }^{\circ} \mathrm{C}$, Run \#1:

Using General Procedure 1, pivalaldehyde (380 $\mu \mathrm{L}, 2.6 \mathrm{mmol})$ was added to a solution of enolate $1(29 \mu \mathrm{L}, 0.26 \mathrm{mmol})$ and adamantane $(5.4 \mathrm{mg}, 0.040 \mathrm{mmol})$ in 1,2-dichloroethane (8.0 $\mathrm{mL}$ ) at $30{ }^{\circ} \mathrm{C}$ as internally monitored. The reaction was monitored every 5 minutes for 90 minutes, and then incrementally thereafter until complete conversion. $\mathrm{k}_{\mathrm{obs}}=0.022 \mathrm{~min}^{-1}$.

\begin{tabular}{|cccccc|}
\hline $\begin{array}{c}\text { Time } \\
(\text { min) }\end{array}$ & $\begin{array}{c}\text { Aldolate } \\
\text { Area }\end{array}$ & $\begin{array}{c}\text { Adamantane } \\
\text { Area }\end{array}$ & Conversion & [Enolate] & In([Enolate]/[Enolate] $)$ \\
\hline \hline 5 & 1527 & 3550 & 0.16 & 0.10 & 0.12 \\
10 & 2561 & 3237 & 0.28 & 0.09 & 0.24 \\
15 & 3852 & 3395 & 0.40 & 0.08 & 0.36 \\
20 & 4421 & 3123 & 0.48 & 0.07 & 0.47 \\
25 & 5203 & 3101 & 0.58 & 0.06 & 0.59 \\
30 & 5564 & 2949 & 0.64 & 0.06 & 0.70 \\
35 & 7145 & 3315 & 0.75 & 0.05 & 0.85 \\
\hline
\end{tabular}


Temperature: $30{ }^{\circ} \mathrm{C}$, Run \#2:

Using General Procedure 1, pivalaldehyde $(1.16 \mathrm{~mL}, 10.4 \mathrm{mmol})$ was added to a solution of enolate $1(150 \mu \mathrm{L}, 1.03 \mathrm{mmol})$ and adamantane $(22 \mathrm{mg}, 0.16 \mathrm{mmol})$ in 1,2-dichloroethane $(8.0 \mathrm{~mL})$ at $30^{\circ} \mathrm{C}$ as internally monitored. The reaction was monitored every 5 minutes for 90 minutes, and then incrementally thereafter until complete conversion. $\mathrm{k}_{\mathrm{obs}}=0.026 \mathrm{~min}^{-1}$.

\begin{tabular}{|c|c|c|c|c|c|}
\hline $\begin{array}{l}\text { Time } \\
(\mathrm{min})\end{array}$ & $\begin{array}{c}\text { Aldolate } \\
\text { Area }\end{array}$ & $\begin{array}{c}\text { Adamantane } \\
\text { Area }\end{array}$ & Conversion & [Enolate] & $\ln \left([\right.$ Enolate $\left.] /[\text { Enolate }]_{0}\right)$ \\
\hline 5 & 3698 & 8134 & 0.17 & 0.10 & 0.14 \\
\hline 10 & 9981 & 11970 & 0.30 & 0.08 & 0.27 \\
\hline 15 & 9753 & 8239 & 0.42 & 0.07 & 0.41 \\
\hline 20 & 11317 & 7846 & 0.50 & 0.07 & 0.53 \\
\hline 25 & 16531 & 9866 & 0.58 & 0.06 & 0.64 \\
\hline 30 & 20130 & 10449 & 0.67 & 0.05 & 0.79 \\
\hline
\end{tabular}

Temperature: $30{ }^{\circ} \mathrm{C}$, Run \#3:

Using General Procedure 1, pivalaldehyde (1.16 mL, $10.4 \mathrm{mmol})$ was added to a solution of enolate $1(150 \mu \mathrm{L}, 1.03 \mathrm{mmol})$ and adamantane $(22 \mathrm{mg}, 0.15 \mathrm{mmol})$ in 1,2-dichloroethane $(8.0 \mathrm{~mL})$ at $30^{\circ} \mathrm{C}$ as internally monitored. The reaction was monitored every 5 minutes for 90 minutes, and then incrementally thereafter until complete conversion. $\mathrm{k}_{\mathrm{obs}}=0.025 \mathrm{~min}^{-1}$.

\begin{tabular}{|c|c|c|c|c|c|}
\hline $\begin{array}{l}\text { Time } \\
(\mathrm{min})\end{array}$ & $\begin{array}{c}\text { Aldolate } \\
\text { Area } \\
\end{array}$ & $\begin{array}{c}\text { Adamantane } \\
\text { Area }\end{array}$ & Conversion & [Enolate] & $\ln \left([\right.$ Enolate $\left.] /[\text { Enolate }]_{0}\right)$ \\
\hline 5 & 4071 & 7921 & 0.19 & 0.10 & 0.14 \\
\hline 10 & 7399 & 7945 & 0.33 & 0.08 & 0.27 \\
\hline 15 & 10888 & 8526 & 0.45 & 0.07 & 0.40 \\
\hline 20 & 14008 & 8708 & 0.56 & 0.07 & 0.53 \\
\hline 25 & 13471 & 7268 & 0.64 & 0.06 & 0.65 \\
\hline 30 & 18727 & 8974 & 0.72 & 0.05 & 0.77 \\
\hline
\end{tabular}


Temperature: $40{ }^{\circ} \mathrm{C}$, Run \#1:

Using General Procedure 1, pivalaldehyde $(380 \mu \mathrm{L}, 2.6 \mathrm{mmol})$ was added to a solution of enolate $1(29 \mu \mathrm{L}, 0.26 \mathrm{mmol})$ and adamantane $(7.1 \mathrm{mg}, 0.052 \mathrm{mmol})$ in 1,2-dichloroethane (2.0 $\mathrm{mL}$ ) at $40{ }^{\circ} \mathrm{C}$ as internally monitored. The reaction was monitored every 5 minutes for 100 minutes, and then incrementally thereafter until complete conversion. $\mathrm{k}_{\mathrm{obs}}=0.035 \mathrm{~min}^{-1}$.

\begin{tabular}{|cccccc|}
\hline $\begin{array}{c}\text { Time } \\
(\mathbf{m i n})\end{array}$ & $\begin{array}{c}\text { Aldolate } \\
\text { Area }\end{array}$ & $\begin{array}{c}\text { Adamantane } \\
\text { Area }\end{array}$ & Conversion & [Enolate] & In([Enolate]/[Enolate] $)$ \\
\hline \hline 5 & 1595 & 3093 & 0.19 & 0.09 & 0.20 \\
10 & 2842 & 3424 & 0.30 & 0.08 & 0.34 \\
18 & 6117 & 4420 & 0.48 & 0.06 & 0.66 \\
22 & 7056 & 4488 & 0.55 & 0.05 & 0.80 \\
26 & 11330 & 6468 & 0.61 & 0.04 & 0.95 \\
30 & 10167 & 5380 & 0.66 & 0.04 & 1.08 \\
35 & 9355 & 4549 & 0.71 & 0.03 & 1.27 \\
\hline
\end{tabular}

Temperature: $40{ }^{\circ} \mathrm{C}$, Run \#2:

Using General Procedure 1, pivalaldehyde $(380 \mu \mathrm{L}, 2.6 \mathrm{mmol})$ was added to a solution of enolate $1(29 \mu \mathrm{L}, 0.26 \mathrm{mmol})$ and adamantane $(6.7 \mathrm{mg}, 0.049 \mathrm{mmol})$ in 1,2-dichloroethane (2.0 $\mathrm{mL}$ ) at $40{ }^{\circ} \mathrm{C}$ as internally monitored. The reaction was monitored every 5 minutes for 90 minutes, and then incrementally thereafter until complete conversion. $\mathrm{k}_{\mathrm{obs}}=0.026 \mathrm{~min}^{-1}$.

\begin{tabular}{|cccccc|}
\hline $\begin{array}{c}\text { Time } \\
(\text { min) }\end{array}$ & $\begin{array}{c}\text { Aldolate } \\
\text { Area }\end{array}$ & $\begin{array}{c}\text { Adamantane } \\
\text { Area }\end{array}$ & Conversion & [Enolate] & In([Enolate]/[Enolate] $)$ \\
\hline \hline 5 & 1326 & 4711 & 0.11 & 0.10 & 0.10 \\
10 & 1776 & 3092 & 0.21 & 0.09 & 0.21 \\
18 & 3695 & 4074 & 0.32 & 0.08 & 0.36 \\
22 & 4210 & 3474 & 0.43 & 0.07 & 0.51 \\
26 & 5539 & 3832 & 0.51 & 0.06 & 0.65 \\
30 & 5972 & 3633 & 0.57 & 0.05 & 0.78 \\
35 & 6005 & 3338 & 0.63 & 0.05 & 0.90 \\
40 & 6595 & 3457 & 0.66 & 0.04 & 0.99 \\
45 & 5750 & 2710 & 0.73 & 0.03 & 1.20 \\
\hline
\end{tabular}




\section{Temperature: $40{ }^{\circ} \mathrm{C}$, Run \#3:}

Using General Procedure 1, pivalaldehyde $(380 \mu \mathrm{L}, 2.6 \mathrm{mmol})$ was added to a solution of enolate $129 \mu \mathrm{L}, 0.26 \mathrm{mmol})$ and adamantane $(22 \mathrm{mg}, 0.15 \mathrm{mmol})$ in 1,2-dichloroethane (2.0 $\mathrm{mL}$ ) at $40{ }^{\circ} \mathrm{C}$ as internally monitored. The reaction was monitored every 3 minutes for 60 minutes, and then incrementally thereafter until complete conversion. $\mathrm{k}_{\mathrm{obs}}=0.025 \mathrm{~min}^{-1}$.

\begin{tabular}{|c|c|c|c|c|c|}
\hline $\begin{array}{l}\text { Time } \\
\text { (min) }\end{array}$ & $\begin{array}{c}\text { Aldolate } \\
\text { Area }\end{array}$ & $\begin{array}{c}\text { Adamantane } \\
\text { Area }\end{array}$ & Conversion & [Enolate] & $\ln \left([\right.$ Enolate $\left.] /[\text { Enolate }]_{o}\right)$ \\
\hline 4 & 1739 & 3834 & 0.17 & 0.10 & 0.13 \\
\hline 7 & 3354 & 4104 & 0.29 & 0.09 & 0.25 \\
\hline 11 & 5481 & 4499 & 0.43 & 0.07 & 0.40 \\
\hline 14 & 6249 & 4244 & 0.51 & 0.07 & 0.51 \\
\hline 17 & 3327 & 1901 & 0.61 & 0.06 & 0.65 \\
\hline 21 & 16019 & 7914 & 0.70 & 0.05 & 0.80 \\
\hline
\end{tabular}

Temperature: $65^{\circ} \mathrm{C}$, Run \#1:

Using General Procedure 1, pivalaldehyde $(760 \mu \mathrm{L}, 5.2 \mathrm{mmol})$ was added to a solution of enolate $1(58 \mu \mathrm{L}, 0.52 \mathrm{mmol})$ and adamantane $(9.1 \mathrm{mg}, 0.07 \mathrm{mmol})$ in 1,2-dichloroethane (8.0 $\mathrm{mL}$ ) at $65{ }^{\circ} \mathrm{C}$ as internally monitored. The reaction was monitored every 4 minutes for 60 minutes, and then incrementally thereafter until complete conversion. $\mathrm{k}_{\mathrm{obs}}=0.085 \mathrm{~min}^{-1}$.

\begin{tabular}{|c|c|c|c|c|c|}
\hline $\begin{array}{l}\text { Time } \\
(\min )\end{array}$ & $\begin{array}{c}\text { Aldolate } \\
\text { Area }\end{array}$ & $\begin{array}{c}\text { Adamantane } \\
\text { Area }\end{array}$ & Conversion & [Enolate] & $\ln \left([\right.$ Enolate $\left.] /[\text { Enolate }]_{0}\right)$ \\
\hline 7 & 6365 & 3494 & 0.45 & 0.06 & 0.60 \\
\hline 14 & 7716 & 2770 & 0.69 & 0.03 & 1.18 \\
\hline 19 & 9388 & 2930 & 0.80 & 0.02 & 1.59 \\
\hline 23 & 7015 & 2028 & 0.86 & 0.02 & 1.96 \\
\hline
\end{tabular}

Temperature: $65^{\circ} \mathrm{C}$, Run \#2:

Using General Procedure 1, pivalaldehyde $(760 \mu \mathrm{L}, 5.2 \mathrm{mmol})$ was added to a solution of enolate $1(58 \mu \mathrm{L}, 0.52 \mathrm{mmol})$ and adamantane $(13 \mathrm{mg}, 0.10 \mathrm{mmol})$ in 1,2-dichloroethane (8.0 $\mathrm{mL})$ at $63{ }^{\circ} \mathrm{C}$ as internally monitored. The reaction was monitored every 4 minutes for 60 minutes, and then incrementally thereafter until complete conversion. $\mathrm{k}_{\mathrm{obs}}=0.055 \mathrm{~min}^{-1}$.

\begin{tabular}{|cccccc|}
\hline $\begin{array}{c}\text { Time } \\
\text { (min) }\end{array}$ & $\begin{array}{c}\text { Aldolate } \\
\text { Area }\end{array}$ & $\begin{array}{c}\text { Adamantane } \\
\text { Area }\end{array}$ & Conversion & [Enolate] & In([Enolate]/[Enolate] $]_{\mathbf{0}}$ ] \\
\hline \hline 5 & 4637 & 5759 & 0.29 & 0.08 & 0.30 \\
10 & 7577 & 4104 & 0.49 & 0.05 & 0.88 \\
18 & 8830 & 4145 & 0.74 & 0.04 & 1.13 \\
23 & 10224 & 4333 & 0.82 & 0.03 & 1.38 \\
\hline
\end{tabular}




\section{Temperature: $65^{\circ} \mathrm{C}$, Run \#3:}

Using General Procedure 1, pivalaldehyde $(760 \mu \mathrm{L}, 5.2 \mathrm{mmol})$ was added to a solution of enolate $1(58 \mu \mathrm{L}, 0.52 \mathrm{mmol})$ and adamantane $(6.8 \mathrm{mg}, 0.05 \mathrm{mmol})$ in 1,2-dichloroethane $(8.0$ $\mathrm{mL}$ ) at $63{ }^{\circ} \mathrm{C}$ as internally monitored. The reaction was monitored every 4 minutes for 60 minutes, and then incrementally thereafter until complete conversion. $\mathrm{k}_{\mathrm{obs}}=0.062 \mathrm{~min}^{-1}$.

\begin{tabular}{|cccccc|}
\hline $\begin{array}{c}\text { Time } \\
\text { (min) }\end{array}$ & $\begin{array}{c}\text { Aldolate } \\
\text { Area }\end{array}$ & $\begin{array}{c}\text { Adamantane } \\
\text { Area }\end{array}$ & Conversion & [Enolate] & In([Enolate]/[Enolate] $]_{\mathbf{o}}$ ) \\
\hline \hline 7 & 6365 & 3494 & 0.41 & 0.07 & 0.52 \\
14 & 7716 & 2770 & 0.62 & 0.04 & 0.98 \\
19 & 9388 & 2930 & 0.72 & 0.03 & 1.26 \\
23 & 7015 & 2028 & 0.77 & 0.03 & 1.49 \\
\hline
\end{tabular}

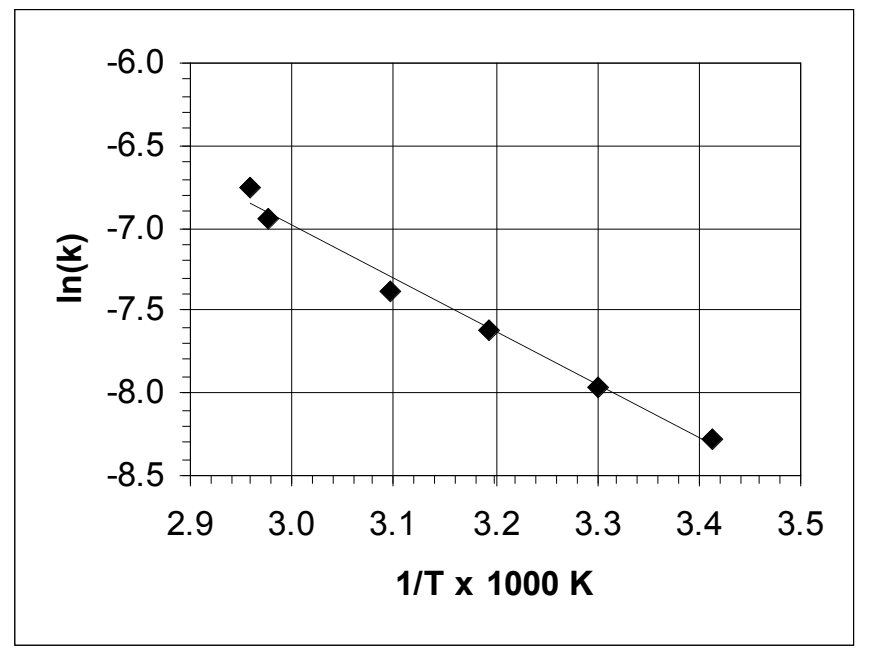

Figure I. Arrhenius Plot. The graph depicts a linear fit, $f(x)=m x+b\left(m=-3.22, R^{2}=0.99\right)$. 


\section{Raw Data. ReactIR Kinetics of Addition of 1 to 2. Uncatalyzed.}

Temperature: $30{ }^{\circ} \mathrm{C}$, Run \#1:

Using General Procedure 2a, pivalaldehyde (300 $\mu \mathrm{L}, 2.7 \mathrm{mmol})$ was added to a solution of enolate $1(38 \mu \mathrm{L}, 0.27 \mathrm{mmol})$ in 1,2-dichloroethane $(1.0 \mathrm{~mL})$ at $30{ }^{\circ} \mathrm{C}$ as internally monitored. The reaction was monitored every 3 minutes until complete conversion. $\mathrm{k}=0.021 \mathrm{M}^{-1} \mathrm{~min}^{-1}$.

\begin{tabular}{|cccccc|}
\hline Time (min) & Absorbance & [Enolate] & Conversion & $\ln ([$ Enolate]) & $\ln \left([\text { Enolate]/[Enolate }]_{\mathbf{o}}\right)$ \\
\hline \hline 0.00 & 0.0314 & 0.203 & 0.00 & 1.59 & 0.00 \\
3.00 & 0.0288 & 0.182 & 0.10 & 1.70 & 0.11 \\
6.00 & 0.0255 & 0.157 & 0.23 & 1.85 & 0.25 \\
9.00 & 0.0229 & 0.136 & 0.33 & 1.99 & 0.40 \\
12.00 & 0.0205 & 0.118 & 0.42 & 2.14 & 0.54 \\
15.00 & 0.0187 & 0.104 & 0.49 & 2.26 & 0.67 \\
18.00 & 0.0167 & 0.089 & 0.56 & 2.42 & 0.82 \\
21.00 & 0.0155 & 0.080 & 0.61 & 2.53 & 0.93 \\
24.00 & 0.0144 & 0.071 & 0.65 & 2.64 & 1.05 \\
27.00 & 0.0131 & 0.061 & 0.70 & 2.80 & 1.21 \\
\hline
\end{tabular}

Temperature: $30{ }^{\circ} \mathrm{C}$, Run \#2:

Using General Procedure 2a, pivalaldehyde $(300 \mu \mathrm{L}, 2.7 \mathrm{mmol})$ was added to a solution of enolate $1(38 \mu \mathrm{L}, 0.27 \mathrm{mmol})$ in 1,2-dichloroethane $(1.0 \mathrm{~mL})$ at $30^{\circ} \mathrm{C}$ as internally monitored. The reaction was monitored every 3 minutes until complete conversion. $\mathrm{k}=0.021 \mathrm{M}^{-1} \mathrm{~min}^{-1}$.

\begin{tabular}{|cccccc|}
\hline Time (min) & Absorbance & [Enolate] & Conversion & $\ln ([$ Enolate]) & $\ln \left([\text { Enolate]/[Enolate }]_{0}\right)$ \\
\hline \hline 0.00 & 0.0274 & 0.203 & 0.00 & 1.59 & 0.00 \\
3.00 & 0.0244 & 0.176 & 0.13 & 1.74 & 0.14 \\
6.00 & 0.0219 & 0.154 & 0.24 & 1.87 & 0.27 \\
9.00 & 0.0195 & 0.133 & 0.34 & 2.02 & 0.42 \\
12.00 & 0.0173 & 0.114 & 0.44 & 2.17 & 0.58 \\
15.00 & 0.0160 & 0.102 & 0.50 & 2.28 & 0.69 \\
18.00 & 0.0143 & 0.087 & 0.57 & 2.44 & 0.84 \\
21.00 & 0.0133 & 0.079 & 0.61 & 2.54 & 0.94 \\
24.00 & 0.0123 & 0.070 & 0.66 & 2.66 & 1.07 \\
27.00 & 0.0113 & 0.061 & 0.70 & 2.79 & 1.20 \\
\hline
\end{tabular}

Temperature: $30^{\circ} \mathrm{C}$, Run \#3:

Using General Procedure 2a, pivalaldehyde (300 $\mu \mathrm{L}, 2.7 \mathrm{mmol})$ was added to a solution of enolate $1(38 \mu \mathrm{L}, 0.27 \mathrm{mmol})$ in 1,2-dichloroethane $(1.0 \mathrm{~mL})$ at $30^{\circ} \mathrm{C}$ as internally monitored. The reaction was monitored every 3 minutes until complete conversion. $\mathrm{k}=0.0211 \mathrm{M}^{-1} \mathrm{~min}^{-1}$. 


\begin{tabular}{|cccccc|}
\hline Time (min) & Absorbance & [Enolate] & Conversion & $\ln ([$ Enolate]) & $\ln \left([\text { Enolate]/[Enolate }]_{\mathbf{o}}\right)$ \\
\hline \hline 0.00 & 0.0353 & 0.203 & 0.00 & 1.595 & 0.00 \\
3.00 & 0.0314 & 0.177 & 0.13 & 1.731 & 0.14 \\
6.00 & 0.0279 & 0.154 & 0.24 & 1.870 & 0.28 \\
9.00 & 0.0249 & 0.134 & 0.34 & 2.012 & 0.42 \\
12.00 & 0.0226 & 0.118 & 0.42 & 2.133 & 0.54 \\
15.00 & 0.0207 & 0.105 & 0.48 & 2.249 & 0.65 \\
18.00 & 0.0187 & 0.092 & 0.54 & 2.381 & 0.79 \\
21.00 & 0.0169 & 0.081 & 0.60 & 2.517 & 0.92 \\
24.00 & 0.0156 & 0.072 & 0.65 & 2.632 & 1.04 \\
27.00 & 0.0143 & 0.063 & 0.69 & 2.766 & 1.17 \\
\hline
\end{tabular}

Temperature: $40^{\circ} \mathrm{C}$, Run \#1:

Using General Procedure 2a, pivalaldehyde $(300 \mu \mathrm{L}, 2.7 \mathrm{mmol})$ was added to a solution of enolate $1(38 \mu \mathrm{L}, 0.27 \mathrm{mmol})$ in 1,2-dichloroethane $(1.0 \mathrm{~mL})$ at $40{ }^{\circ} \mathrm{C}$ as internally monitored. The reaction was monitored every 3 minutes until complete conversion. $\mathrm{k}=0.027 \mathrm{M}^{-1} \mathrm{~min}^{-1}$.

\begin{tabular}{|cccccc|}
\hline Time $(\mathbf{m i n})$ & Absorbance & [Enolate] & Conversion & $\ln ([$ Enolate]) & $\ln \left([\text { Enolate]/[Enolate }]_{\mathbf{o}}\right)$ \\
\hline \hline 0.00 & 0.0342 & 0.203 & 0.00 & 1.59 & 0.00 \\
3.00 & 0.0291 & 0.168 & 0.17 & 1.78 & 0.19 \\
6.00 & 0.0251 & 0.141 & 0.31 & 1.96 & 0.37 \\
9.00 & 0.0219 & 0.119 & 0.42 & 2.13 & 0.54 \\
12.00 & 0.0192 & 0.099 & 0.51 & 2.31 & 0.71 \\
15.00 & 0.0169 & 0.084 & 0.59 & 2.48 & 0.88 \\
18.00 & 0.0153 & 0.073 & 0.64 & 2.62 & 1.02 \\
21.00 & 0.0137 & 0.062 & 0.69 & 2.78 & 1.19 \\
\hline
\end{tabular}

Temperature: $40{ }^{\circ} \mathrm{C}$, Run \#2:

Using General Procedure 2a, pivalaldehyde (300 $\mu \mathrm{L}, 2.7 \mathrm{mmol})$ was added to a solution of enolate $1(38 \mu \mathrm{L}, 0.27 \mathrm{mmol})$ in 1,2-dichloroethane $(1.0 \mathrm{~mL})$ at $40^{\circ} \mathrm{C}$ as internally monitored. The reaction was monitored every 2 minutes until complete conversion. $\mathrm{k}=0.028 \mathrm{M}^{-1} \mathrm{~min}^{-1}$.

\begin{tabular}{|cccccc|}
\hline Time (min) & Absorbance & [Enolate] & Conversion & $\ln ([$ Enolate]) & $\ln \left([\text { Enolate]/[Enolate }]_{\mathbf{o}}\right)$ \\
\hline \hline 0.00 & 0.0306 & 0.203 & 0.00 & 1.59 & 0.00 \\
2.00 & 0.0276 & 0.180 & 0.11 & 1.72 & 0.12 \\
4.00 & 0.0252 & 0.161 & 0.21 & 1.83 & 0.23 \\
6.00 & 0.0229 & 0.143 & 0.30 & 1.95 & 0.35 \\
8.00 & 0.0208 & 0.127 & 0.38 & 2.07 & 0.47 \\
10.00 & 0.0190 & 0.113 & 0.44 & 2.18 & 0.59 \\
12.00 & 0.0174 & 0.101 & 0.50 & 2.30 & 0.70 \\
14.00 & 0.0160 & 0.090 & 0.56 & 2.41 & 0.81 \\
16.00 & 0.0148 & 0.081 & 0.60 & 2.52 & 0.92 \\
18.00 & 0.0136 & 0.071 & 0.65 & 2.64 & 1.05 \\
20.00 & 0.0128 & 0.065 & 0.68 & 2.729 & 1.134 \\
\hline
\end{tabular}


Temperature: $40{ }^{\circ} \mathrm{C}$, Run \#3:

Using General Procedure 2a, pivalaldehyde (300 $\mu \mathrm{L}, 2.7 \mathrm{mmol})$ was added to a solution of enolate $1(38 \mu \mathrm{L}, 0.27 \mathrm{mmol})$ in 1,2-dichloroethane $(1.0 \mathrm{~mL})$ at $40{ }^{\circ} \mathrm{C}$ as internally monitored. The reaction was monitored every 2 minutes until complete conversion. $\mathrm{k}=0.0266 \mathrm{M}^{-1} \mathrm{~min}^{-1}$.

\begin{tabular}{|cccccc|}
\hline Time (min) & Absorbance & [Enolate] & Conversion & $\ln ([$ Enolate]) & $\ln \left([\text { Enolate]/[Enolate }]_{\mathbf{o}}\right)$ \\
\hline \hline 0.00 & 0.0321 & 0.203 & 0.00 & 1.59 & 0.00 \\
2.00 & 0.0291 & 0.181 & 0.11 & 1.71 & 0.11 \\
4.00 & 0.0262 & 0.160 & 0.21 & 1.83 & 0.24 \\
6.00 & 0.0239 & 0.143 & 0.30 & 1.94 & 0.35 \\
8.00 & 0.0215 & 0.125 & 0.38 & 2.08 & 0.48 \\
10.00 & 0.0197 & 0.112 & 0.45 & 2.19 & 0.60 \\
12.00 & 0.0181 & 0.100 & 0.51 & 2.30 & 0.70 \\
14.00 & 0.0166 & 0.089 & 0.56 & 2.42 & 0.82 \\
16.00 & 0.0154 & 0.081 & 0.60 & 2.52 & 0.92 \\
18.00 & 0.0145 & 0.074 & 0.64 & 2.60 & 1.01 \\
20.00 & 0.0134 & 0.065 & 0.68 & 2.72 & 1.13 \\
22.00 & 0.0128 & 0.061 & 0.70 & 2.79 & 1.19 \\
\hline
\end{tabular}

Temperature: $50{ }^{\circ} \mathrm{C}$, Run \#1:

Using General Procedure 2a, pivalaldehyde $(300 \mu \mathrm{L}, 2.7 \mathrm{mmol})$ was added to a solution of enolate $1(38 \mu \mathrm{L}, 0.27 \mathrm{mmol})$ in 1,2-dichloroethane $(1.0 \mathrm{~mL})$ at $50{ }^{\circ} \mathrm{C}$ as internally monitored. The reaction was monitored every 2 minutes until complete conversion. $\mathrm{k}=0.0407 \mathrm{M}^{-1} \mathrm{~min}^{-1}$.

\begin{tabular}{|cccccc|}
\hline Time (min) & Absorbance & [Enolate] & Conversion & $\ln ([$ Enolate]) & $\ln \left([\text { Enolate]/[Enolate }]_{0}\right)$ \\
\hline \hline 0.00 & 0.0369 & 0.203 & 0.00 & 1.59 & 0.00 \\
2.00 & 0.0302 & 0.160 & 0.21 & 1.84 & 0.24 \\
4.00 & 0.0263 & 0.134 & 0.34 & 2.01 & 0.42 \\
6.00 & 0.0231 & 0.113 & 0.44 & 2.18 & 0.58 \\
8.00 & 0.0206 & 0.097 & 0.52 & 2.33 & 0.73 \\
10.00 & 0.0183 & 0.082 & 0.60 & 2.50 & 0.90 \\
12.00 & 0.0166 & 0.071 & 0.65 & 2.65 & 1.05 \\
14.00 & 0.0148 & 0.059 & 0.71 & 2.82 & 1.23 \\
\hline
\end{tabular}


Temperature: $50{ }^{\circ} \mathrm{C}$, Run \#2:

Using General Procedure 2a, pivalaldehyde (300 $\mu \mathrm{L}, 2.7 \mathrm{mmol})$ was added to a solution of enolate $1(38 \mu \mathrm{L}, 0.27 \mathrm{mmol})$ in 1,2-dichloroethane $(1.0 \mathrm{~mL})$ at $50{ }^{\circ} \mathrm{C}$ as internally monitored. The reaction was monitored every 2 minutes until complete conversion. $\mathrm{k}=0.0385 \mathrm{M}^{-1} \mathrm{~min}^{-1}$.

\begin{tabular}{|cccccc|}
\hline Time (min) & Absorbance & [Enolate] & Conversion & $\ln ([$ Enolate]) & $\ln \left([\text { Enolate]/[Enolate }]_{\mathbf{o}}\right)$ \\
\hline \hline 0.00 & 0.0321 & 0.203 & 0.00 & 1.59 & 0.00 \\
2.00 & 0.0282 & 0.173 & 0.15 & 1.76 & 0.16 \\
4.00 & 0.0244 & 0.144 & 0.29 & 1.94 & 0.34 \\
6.00 & 0.0217 & 0.124 & 0.39 & 2.09 & 0.49 \\
8.00 & 0.0194 & 0.106 & 0.48 & 2.24 & 0.65 \\
10.00 & 0.0172 & 0.090 & 0.56 & 2.41 & 0.81 \\
12.00 & 0.0155 & 0.077 & 0.62 & 2.56 & 0.97 \\
14.00 & 0.0141 & 0.067 & 0.67 & 2.71 & 1.11 \\
\hline
\end{tabular}

Temperature: $50^{\circ} \mathrm{C}$, Run \#3:

Using General Procedure 2a, pivalaldehyde $(300 \mu \mathrm{L}, 2.7 \mathrm{mmol})$ was added to a solution of enolate $1(38 \mu \mathrm{L}, 0.27 \mathrm{mmol})$ in 1,2-dichloroethane $(1.0 \mathrm{~mL})$ at $50{ }^{\circ} \mathrm{C}$ as internally monitored. The reaction was monitored every 2 minutes until complete conversion. $\mathrm{k}=0.0417 \mathrm{M}^{-1} \mathrm{~min}^{-1}$.

\begin{tabular}{|cccccc|}
\hline Time (min) & Absorbance & [Enolate] & Conversion & $\ln ([$ Enolate]) & $\ln \left([\text { Enolate]/[Enolate }]_{\mathbf{o}}\right)$ \\
\hline \hline 0.00 & 0.0338 & 0.203 & 0.00 & 1.59 & 0.00 \\
2.00 & 0.0300 & 0.175 & 0.14 & 1.74 & 0.15 \\
4.00 & 0.0265 & 0.150 & 0.26 & 1.90 & 0.30 \\
6.00 & 0.0231 & 0.125 & 0.38 & 2.08 & 0.49 \\
8.00 & 0.0204 & 0.105 & 0.48 & 2.25 & 0.66 \\
10.00 & 0.0180 & 0.088 & 0.57 & 2.43 & 0.84 \\
12.00 & 0.0160 & 0.073 & 0.64 & 2.61 & 1.02 \\
14.00 & 0.0144 & 0.062 & 0.70 & 2.79 & 1.19 \\
\hline
\end{tabular}

Temperature: $60{ }^{\circ} \mathrm{C}$, Run \#1:

Using General Procedure 2a, pivalaldehyde $(300 \mu \mathrm{L}, 2.7 \mathrm{mmol})$ was added to a solution of enolate $1(38 \mu \mathrm{L}, 0.27 \mathrm{mmol})$ in 1,2-dichloroethane $(1.0 \mathrm{~mL})$ at $60^{\circ} \mathrm{C}$ as internally monitored. The reaction was monitored every 2 minutes until complete conversion. $\mathrm{k}=0.0550 \mathrm{M}^{-1} \mathrm{~min}^{-1}$.

\begin{tabular}{|cccccc|}
\hline Time (min) & Absorbance & [Enolate] & Conversion & $\ln ([$ Enolate]) & $\ln \left([\text { Enolate]/[Enolate }]_{\mathbf{o}}\right)$ \\
\hline \hline 0.00 & 0.0292 & 0.203 & 0.00 & 1.59 & 4.93 \\
2.00 & 0.0246 & 0.164 & 0.19 & 1.81 & 6.11 \\
4.00 & 0.0207 & 0.131 & 0.36 & 2.03 & 7.65 \\
6.00 & 0.0179 & 0.107 & 0.47 & 2.24 & 9.38 \\
8.00 & 0.0153 & 0.085 & 0.58 & 2.47 & 11.83 \\
10.00 & 0.0131 & 0.066 & 0.68 & 2.72 & 15.18 \\
\hline
\end{tabular}


Temperature: $60{ }^{\circ} \mathrm{C}$, Run \#2:

Using General Procedure 2a, pivalaldehyde (300 $\mu \mathrm{L}, 2.7 \mathrm{mmol})$ was added to a solution of enolate $1(38 \mu \mathrm{L}, 0.27 \mathrm{mmol})$ in 1,2-dichloroethane $(1.0 \mathrm{~mL})$ at $60{ }^{\circ} \mathrm{C}$ as internally monitored. The reaction was monitored every 1 minutes until complete conversion. $\mathrm{k}=0.0545 \mathrm{M}^{-1} \mathrm{~min}^{-1}$.

\begin{tabular}{|cccccc|}
\hline Time (min) & Absorbance & [Enolate] & Conversion & $\ln ([$ Enolate]) & $\ln \left([\text { Enolate]/[Enolate }]_{\mathbf{o}}\right)$ \\
\hline \hline 0.00 & 0.0004 & 0.000 & 0.00 & & \\
0.00 & 0.0005 & 0.000 & 0.00 & & 0.00 \\
0.00 & 0.0229 & 0.203 & 0.00 & 1.59 & 0.10 \\
1.00 & 0.0208 & 0.184 & 0.09 & 1.69 & 0.22 \\
2.00 & 0.0184 & 0.162 & 0.20 & 1.82 & 0.32 \\
3.00 & 0.0167 & 0.147 & 0.28 & 1.92 & 0.45 \\
4.00 & 0.0147 & 0.129 & 0.36 & 2.05 & 0.56 \\
5.00 & 0.0132 & 0.115 & 0.43 & 2.16 & 0.66 \\
6.00 & 0.0120 & 0.104 & 0.49 & 2.26 & 0.80 \\
7.00 & 0.0105 & 0.091 & 0.55 & 2.39 & 0.93 \\
8.00 & 0.0093 & 0.080 & 0.61 & 2.52 & 0.99 \\
9.00 & 0.0087 & 0.075 & 0.63 & 2.59 & 1.11 \\
10.00 & 0.0079 & 0.067 & 0.67 & 2.70 & 1.25 \\
11.00 & 0.0069 & 0.058 & 0.71 & 2.84 & \\
\hline
\end{tabular}

Temperature: $60{ }^{\circ} \mathrm{C}$, Run \#3:

Using General Procedure 2a, pivalaldehyde $(300 \mu \mathrm{L}, 2.7 \mathrm{mmol})$ was added to a solution of enolate $1(38 \mu \mathrm{L}, 0.27 \mathrm{mmol})$ in 1,2-dichloroethane $(1.0 \mathrm{~mL})$ at $60^{\circ} \mathrm{C}$ as internally monitored. The reaction was monitored every 1 minutes until complete conversion. $\mathrm{k}=0.0532 \mathrm{M}^{-1} \mathrm{~min}^{-1}$.

\begin{tabular}{|cccccc|}
\hline Time $(\mathbf{m i n})$ & Absorbance & [Enolate] & Conversion & $\ln ([$ Enolate]) & $\ln \left([\text { Enolate]/[Enolate }]_{0}\right)$ \\
\hline \hline 0.00 & 0.0287 & 0.203 & 0.00 & 1.59 & 0.00 \\
1.00 & 0.0263 & 0.183 & 0.10 & 1.70 & 0.10 \\
2.00 & 0.0238 & 0.161 & 0.21 & 1.83 & 0.23 \\
3.00 & 0.0216 & 0.142 & 0.30 & 1.95 & 0.36 \\
4.00 & 0.0201 & 0.129 & 0.36 & 2.05 & 0.45 \\
5.00 & 0.0182 & 0.113 & 0.44 & 2.18 & 0.59 \\
6.00 & 0.0172 & 0.104 & 0.49 & 2.26 & 0.67 \\
7.00 & 0.0161 & 0.094 & 0.53 & 2.36 & 0.77 \\
8.00 & 0.0149 & 0.084 & 0.58 & 2.47 & 0.88 \\
9.00 & 0.0139 & 0.075 & 0.63 & 2.59 & 0.99 \\
10.00 & 0.0132 & 0.069 & 0.66 & 2.67 & 1.08 \\
11.00 & 0.0121 & 0.060 & 0.70 & 2.81 & 1.22 \\
\hline
\end{tabular}




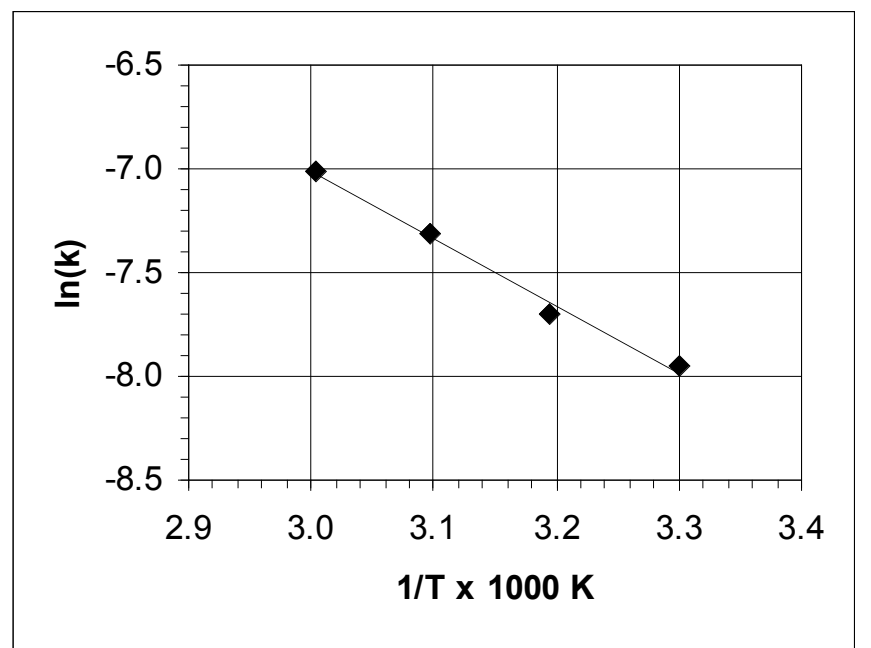

Figure II. Arrhenius Plot. The graph depicts a linear fit, $f(x)=m x+b\left(m=-3.25, R^{2}=0.99\right)$. 
Raw Data. ReactIR Kinetics of Addition of 4 to 5a. Uncatalyzed.

Temperature: $30^{\circ} \mathrm{C}$, Run \#1:

Using General Procedure 2a, benzaldehyde (46 $\mu \mathrm{L}, 0.45 \mathrm{mmol}$ ) was added to a solution of enolate $4(82 \mu \mathrm{L}, 0.45 \mathrm{mmol})$ in 1,2-dichloroethane $(1.5 \mathrm{~mL})$ at $30{ }^{\circ} \mathrm{C}$ as internally monitored. The reaction was monitored every 1 minute until complete conversion. $\mathrm{k}=1.741 \mathrm{M}^{-1} \mathrm{~min}^{-1}$.

\begin{tabular}{|ccccc|}
\hline Time (min) & Absorbance & [Enolate] & Conversion & 1/[Enolate] \\
\hline \hline 0.00 & 0.0325 & 0.276 & 0.00 & 3.62 \\
1.00 & 0.0241 & 0.192 & 0.31 & 5.22 \\
2.00 & 0.0191 & 0.142 & 0.49 & 7.05 \\
3.00 & 0.0163 & 0.113 & 0.59 & 8.81 \\
4.00 & 0.0130 & 0.080 & 0.71 & 12.54 \\
5.00 & 0.0127 & 0.077 & 0.72 & 13.04 \\
\hline
\end{tabular}

\section{Temperature: $30{ }^{\circ} \mathrm{C}$, Run \#2:}

Using General Procedure 2a, benzaldehyde ( $46 \mu \mathrm{L}, 0.45 \mathrm{mmol}$ ) was added to a solution of enolate $4(82 \mu \mathrm{L}, 0.45 \mathrm{mmol})$ in 1,2 -dichloroethane $(1.5 \mathrm{~mL})$ at $30^{\circ} \mathrm{C}$ as internally monitored. The reaction was monitored every 1 minute until complete conversion. $\mathrm{k}=1.732 \mathrm{M}^{-1} \mathrm{~min}^{-1}$.

\begin{tabular}{|ccccc|}
\hline Time (min) & Absorbance & [Enolate] & Conversion & 1/[Enolate] \\
\hline \hline 0.00 & 0.0352 & 0.276 & 0.00 & 3.62 \\
1.00 & 0.0264 & 0.195 & 0.29 & 5.13 \\
2.00 & 0.0208 & 0.144 & 0.48 & 6.97 \\
3.00 & 0.0176 & 0.114 & 0.59 & 8.78 \\
4.00 & 0.0151 & 0.091 & 0.67 & 11.03 \\
5.00 & 0.0135 & 0.077 & 0.72 & 13.07 \\
\hline
\end{tabular}

Temperature: $30{ }^{\circ} \mathrm{C}$, Run \#3:

Using General Procedure 2a, benzaldehyde (46 $\mu \mathrm{L}, 0.45 \mathrm{mmol})$ was added to a solution of enolate $4(82 \mu \mathrm{L}, 0.45 \mathrm{mmol})$ in 1,2 -dichloroethane $(1.5 \mathrm{~mL})$ at $30^{\circ} \mathrm{C}$ as internally monitored. The reaction was monitored every 1 minute until complete conversion. $\mathrm{k}=1.662 \mathrm{M}^{-1} \mathrm{~min}^{-1}$.

\begin{tabular}{|ccccc|}
\hline Time (min) & Absorbance & [Enolate] & Conversion & 1/[Enolate] \\
\hline \hline 0.00 & 0.0336 & 0.276 & 0.00 & 3.62 \\
1.00 & 0.0259 & 0.200 & 0.28 & 5.00 \\
2.00 & 0.0205 & 0.146 & 0.47 & 6.83 \\
3.00 & 0.0175 & 0.117 & 0.58 & 8.55 \\
4.00 & 0.0153 & 0.095 & 0.66 & 10.50 \\
5.00 & 0.0131 & 0.074 & 0.73 & 13.54 \\
\hline
\end{tabular}


Temperature: $40{ }^{\circ} \mathrm{C}$, Run \#1:

Using General Procedure 2a, benzaldehyde (46 $\mu \mathrm{L}, 0.45 \mathrm{mmol})$ was added to a solution of enolate $4(82 \mu \mathrm{L}, 0.45 \mathrm{mmol})$ in 1,2 -dichloroethane $(1.5 \mathrm{~mL})$ at $40{ }^{\circ} \mathrm{C}$ as internally monitored. The reaction was monitored every 1 minute until complete conversion. $\mathrm{k}=1.816 \mathrm{M}^{-1} \mathrm{~min}^{-1}$.

\begin{tabular}{|ccccc|}
\hline Time (min) & Absorbance & [Enolate] & Conversion & 1/[Enolate] \\
\hline \hline 0.00 & 0.0290 & 0.276 & 0.00 & 3.62 \\
1.00 & 0.0273 & 0.258 & 0.07 & 3.87 \\
2.00 & 0.0196 & 0.178 & 0.36 & 5.63 \\
3.00 & 0.0153 & 0.132 & 0.52 & 7.56 \\
4.00 & 0.0130 & 0.108 & 0.61 & 9.28 \\
5.00 & 0.0112 & 0.089 & 0.68 & 11.20 \\
6.00 & 0.0102 & 0.078 & 0.72 & 12.78 \\
\hline
\end{tabular}

Temperature: $40{ }^{\circ} \mathrm{C}$, Run \#2:

Using General Procedure 2a, benzaldehyde (46 $\mu \mathrm{L}, 0.45 \mathrm{mmol}$ ) was added to a solution of enolate $4(82 \mu \mathrm{L}, 0.45 \mathrm{mmol})$ in 1,2-dichloroethane $(1.5 \mathrm{~mL})$ at $40{ }^{\circ} \mathrm{C}$ as internally monitored. The reaction was monitored every 1 minute until complete conversion. $\mathrm{k}=1.807 \mathrm{M}^{-1} \mathrm{~min}^{-1}$.

\begin{tabular}{|ccccc|}
\hline Time (min) & Absorbance & [Enolate] & Conversion & 1/[Enolate] \\
\hline \hline 0.00 & 0.0317 & 0.276 & 0.00 & 3.62 \\
1.00 & 0.0297 & 0.257 & 0.07 & 3.90 \\
2.00 & 0.0219 & 0.181 & 0.35 & 5.54 \\
3.00 & 0.0171 & 0.134 & 0.52 & 7.48 \\
4.00 & 0.0145 & 0.108 & 0.61 & 9.27 \\
5.00 & 0.0124 & 0.087 & 0.68 & 11.44 \\
6.00 & 0.0110 & 0.074 & 0.73 & 13.57 \\
\hline
\end{tabular}

\section{Temperature: $40{ }^{\circ} \mathrm{C}$, Run \#3:}

Using General Procedure 2a, benzaldehyde (46 $\mu \mathrm{L}, 0.45 \mathrm{mmol}$ ) was added to a solution of enolate $4(82 \mu \mathrm{L}, 0.45 \mathrm{mmol})$ in 1,2-dichloroethane $(1.5 \mathrm{~mL})$ at $40^{\circ} \mathrm{C}$ as internally monitored. The reaction was monitored every 1 minute until complete conversion. $\mathrm{k}=1.807 \mathrm{M}^{-1} \mathrm{~min}^{-1}$.

\begin{tabular}{|ccccc|}
\hline Time (min) & Absorbance & [Enolate] & Conversion & 1/[Enolate] \\
\hline \hline 0.00 & 0.0296 & 0.276 & 0.00 & 3.62 \\
1.00 & 0.0284 & 0.263 & 0.05 & 3.80 \\
2.00 & 0.0210 & 0.184 & 0.33 & 5.42 \\
3.00 & 0.0162 & 0.135 & 0.51 & 7.42 \\
4.00 & 0.0134 & 0.105 & 0.62 & 9.53 \\
5.00 & 0.0109 & 0.079 & 0.72 & 12.70 \\
6.00 & 0.0102 & 0.071 & 0.74 & 14.09 \\
\hline
\end{tabular}


Temperature: $50{ }^{\circ} \mathrm{C}$, Run \#1:

Using General Procedure 2a, benzaldehyde (46 $\mu \mathrm{L}, 0.45 \mathrm{mmol}$ ) was added to a solution of enolate $4(82 \mu \mathrm{L}, 0.45 \mathrm{mmol})$ in 1,2 -dichloroethane $(1.5 \mathrm{~mL})$ at $50{ }^{\circ} \mathrm{C}$ as internally monitored. The reaction was monitored every 30 seconds until complete conversion. $\mathrm{k}=1.811 \mathrm{M}^{-1} \mathrm{~min}^{-1}$.

\begin{tabular}{|ccccc|}
\hline Time (min) & Absorbance & [Enolate] & Conversion & 1/[Enolate] \\
\hline \hline 0.00 & 0.0375 & 0.276 & 0.00 & 3.62 \\
0.50 & 0.0326 & 0.222 & 0.19 & 4.50 \\
1.00 & 0.0294 & 0.187 & 0.32 & 5.36 \\
1.50 & 0.0269 & 0.159 & 0.42 & 6.28 \\
2.00 & 0.0250 & 0.139 & 0.50 & 7.20 \\
2.50 & 0.0236 & 0.123 & 0.55 & 8.12 \\
3.00 & 0.0222 & 0.108 & 0.61 & 9.22 \\
3.50 & 0.0215 & 0.100 & 0.64 & 9.96 \\
4.00 & 0.0209 & 0.093 & 0.66 & 10.72 \\
4.50 & 0.0198 & 0.082 & 0.70 & 12.21 \\
\hline
\end{tabular}

\section{Temperature: $50^{\circ} \mathrm{C}$, Run \#2:}

Using General Procedure 2a, benzaldehyde (46 $\mu \mathrm{L}, 0.45 \mathrm{mmol})$ was added to a solution of enolate $4(82 \mu \mathrm{L}, 0.45 \mathrm{mmol})$ in 1,2 -dichloroethane $(1.5 \mathrm{~mL})$ at $50{ }^{\circ} \mathrm{C}$ as internally monitored. The reaction was monitored every 30 seconds until complete conversion. $\mathrm{k}=2.062 \mathrm{M}^{-1} \mathrm{~min}^{-1}$.

\begin{tabular}{|ccccc|}
\hline Time (min) & Absorbance & [Enolate] & Conversion & 1/[Enolate] \\
\hline \hline 0.00 & 0.0288 & 0.276 & 0.00 & 3.62 \\
0.50 & 0.0276 & 0.264 & 0.05 & 3.79 \\
1.00 & 0.0225 & 0.212 & 0.23 & 4.72 \\
1.50 & 0.0191 & 0.177 & 0.36 & 5.65 \\
2.00 & 0.0161 & 0.146 & 0.47 & 6.87 \\
2.50 & 0.0142 & 0.127 & 0.54 & 7.88 \\
3.00 & 0.0128 & 0.113 & 0.59 & 8.88 \\
3.50 & 0.0117 & 0.101 & 0.64 & 9.95 \\
4.00 & 0.0102 & 0.086 & 0.69 & 11.65 \\
4.50 & 0.0096 & 0.079 & 0.71 & 12.63 \\
\hline
\end{tabular}

\section{Temperature: $50{ }^{\circ} \mathrm{C}$, Run \#3:}

Using General Procedure 2a, benzaldehyde (46 $\mu \mathrm{L}, 0.45 \mathrm{mmol})$ was added to a solution of enolate $4(82 \mu \mathrm{L}, 0.45 \mathrm{mmol})$ in 1,2 -dichloroethane $(1.5 \mathrm{~mL})$ at $50{ }^{\circ} \mathrm{C}$ as internally monitored. The reaction was monitored every 30 seconds until complete conversion. $\mathrm{k}=2.227 \mathrm{M}^{-1} \mathrm{~min}^{-1}$. 


\begin{tabular}{|ccccc|}
\hline Time (min) & Absorbance & [Enolate] & Conversion & 1/[Enolate] \\
\hline \hline 0.00 & 0.0291 & 0.276 & 0.00 & 3.62 \\
0.50 & 0.0277 & 0.262 & 0.05 & 3.82 \\
1.00 & 0.0227 & 0.210 & 0.24 & 4.75 \\
1.50 & 0.0190 & 0.172 & 0.38 & 5.80 \\
2.00 & 0.0160 & 0.143 & 0.48 & 7.02 \\
2.50 & 0.0139 & 0.121 & 0.56 & 8.25 \\
3.00 & 0.0126 & 0.107 & 0.61 & 9.32 \\
3.50 & 0.0114 & 0.095 & 0.66 & 10.55 \\
4.00 & 0.0099 & 0.079 & 0.71 & 12.58 \\
\hline
\end{tabular}

Temperature: $60^{\circ} \mathrm{C}$, Run \#1:

Using General Procedure 2a, benzaldehyde ( $46 \mu \mathrm{L}, 0.45 \mathrm{mmol}$ ) was added to a solution of enolate $4(82 \mu \mathrm{L}, 0.45 \mathrm{mmol})$ in 1,2-dichloroethane $(1.5 \mathrm{~mL})$ at $60^{\circ} \mathrm{C}$ as internally monitored. The reaction was monitored every 30 seconds until complete conversion. $\mathrm{k}=2.541 \mathrm{M}^{-1} \mathrm{~min}^{-1}$.

\begin{tabular}{|ccccc|}
\hline Time (min) & Absorbance & [Enolate] & Conversion & 1/[Enolate] \\
\hline \hline 0.00 & 0.0239 & 0.276 & 0.00 & 3.62 \\
0.50 & 0.0194 & 0.210 & 0.24 & 4.75 \\
1.00 & 0.0166 & 0.171 & 0.38 & 5.86 \\
1.50 & 0.0143 & 0.137 & 0.51 & 7.32 \\
2.00 & 0.0128 & 0.115 & 0.58 & 8.69 \\
2.50 & 0.0113 & 0.093 & 0.66 & 10.70 \\
3.00 & 0.0107 & 0.086 & 0.69 & 11.69 \\
\hline
\end{tabular}

\section{Temperature: $60^{\circ} \mathrm{C}$, Run \#2:}

Using General Procedure 2a, benzaldehyde ( $46 \mu \mathrm{L}, 0.45 \mathrm{mmol}$ ) was added to a solution of enolate $4(82 \mu \mathrm{L}, 0.45 \mathrm{mmol})$ in 1,2-dichloroethane $(1.5 \mathrm{~mL})$ at $60^{\circ} \mathrm{C}$ as internally monitored. The reaction was monitored every 30 seconds until complete conversion. $\mathrm{k}=2.627 \mathrm{M}^{-2} \min ^{-1}$.

\begin{tabular}{|ccccc|}
\hline Time (min) & Absorbance & [Enolate] & Conversion & 1/[Enolate] \\
\hline \hline 0.00 & 0.0281 & 0.276 & 0.00 & 3.62 \\
0.50 & 0.0255 & 0.249 & 0.10 & 4.02 \\
1.00 & 0.0197 & 0.186 & 0.33 & 5.37 \\
1.50 & 0.0160 & 0.146 & 0.47 & 6.84 \\
2.00 & 0.0129 & 0.114 & 0.59 & 8.78 \\
2.50 & 0.0111 & 0.095 & 0.66 & 10.57 \\
3.00 & 0.0100 & 0.082 & 0.70 & 12.12 \\
\hline
\end{tabular}




\section{Temperature: $60{ }^{\circ} \mathrm{C}$, Run \#3:}

Using General Procedure 2a, benzaldehyde ( $46 \mu \mathrm{L}, 0.45 \mathrm{mmol}$ ) was added to a solution of enolate $4(82 \mu \mathrm{L}, 0.45 \mathrm{mmol})$ in 1,2-dichloroethane $(1.5 \mathrm{~mL})$ at $60{ }^{\circ} \mathrm{C}$ as internally monitored. The reaction was monitored every 30 seconds until complete conversion. $\mathrm{k}=2.956 \mathrm{M}^{-2} \mathrm{~min}^{-1}$.

\begin{tabular}{|ccccc|}
\hline Time (min) & Absorbance & [Enolate] & Conversion & 1/[Enolate] \\
\hline \hline 0.00 & 0.0276 & 0.276 & 0.00 & 3.62 \\
0.50 & 0.0248 & 0.247 & 0.11 & 4.05 \\
1.00 & 0.0194 & 0.189 & 0.31 & 5.28 \\
1.50 & 0.0151 & 0.144 & 0.48 & 6.94 \\
2.00 & 0.0127 & 0.119 & 0.57 & 8.43 \\
2.50 & 0.0104 & 0.095 & 0.66 & 10.57 \\
3.00 & 0.0093 & 0.083 & 0.70 & 12.06 \\
\hline
\end{tabular}

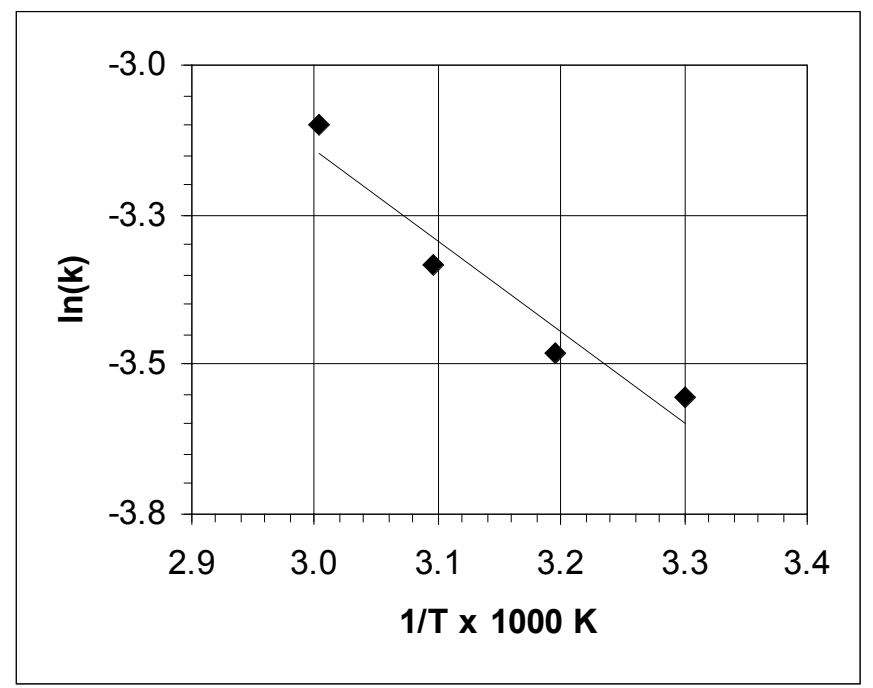

Figure III. Arrhenius Plot. The graph depicts a linear fit, $f(x)=m x+b\left(m=-1.52, R^{2}=0.94\right)$. 
Raw Data. ReactIR Kinetics of Addition of 4 to 5a. Catalyzed by ( \pm )-7j.

Temperature: $-49{ }^{\circ} \mathrm{C}$, Run \#1:

Using General Procedure $2 \mathrm{~b}$, benzaldehyde $(62 \mu \mathrm{L}, 0.61 \mathrm{mmol})$ was added to a solution of enolate $4(111 \mu \mathrm{L}, 0.61 \mathrm{mmol})$ and phosphoramide $( \pm)-7 \mathbf{j}$ (15 mg, $0.031 \mathrm{mmol})$ in methylene chloride $(2.3 \mathrm{~mL})$ at $-49{ }^{\circ} \mathrm{C}$ as internally monitored. The reaction was monitored every 30 seconds until complete conversion. $\mathrm{k}=55.57 \mathrm{M}^{-2} \min ^{-1}$.

\begin{tabular}{|ccccc|}
\hline Time $(\mathbf{m i n})$ & Absorbance & [Enolate] & Conversion & 1/[Enolate] \\
\hline \hline 0.0 & 0.0325 & 0.246 & 0.00 & 4.06 \\
0.5 & 0.2473 & 0.000 & 0.00 & 0.00 \\
1.0 & 0.0750 & 0.000 & 0.00 & 0.00 \\
1.5 & 0.0292 & 0.219 & 0.11 & 4.56 \\
2.0 & 0.0275 & 0.205 & 0.17 & 4.87 \\
2.5 & 0.0258 & 0.191 & 0.22 & 5.24 \\
3.0 & 0.0246 & 0.181 & 0.26 & 5.52 \\
3.5 & 0.0232 & 0.170 & 0.31 & 5.89 \\
4.0 & 0.0218 & 0.158 & 0.36 & 6.33 \\
4.5 & 0.0204 & 0.147 & 0.40 & 6.82 \\
5.0 & 0.0197 & 0.141 & 0.43 & 7.10 \\
5.5 & 0.0188 & 0.134 & 0.46 & 7.47 \\
6.0 & 0.0183 & 0.130 & 0.47 & 7.71 \\
6.5 & 0.0177 & 0.125 & 0.49 & 8.02 \\
7.0 & 0.0166 & 0.115 & 0.53 & 8.67 \\
7.5 & 0.0162 & 0.112 & 0.55 & 8.93 \\
8.0 & 0.0154 & 0.105 & 0.57 & 9.49 \\
8.5 & 0.0149 & 0.101 & 0.59 & 9.86 \\
9.0 & 0.0144 & 0.097 & 0.61 & 10.29 \\
9.5 & 0.0138 & 0.093 & 0.62 & 10.81 \\
10.0 & 0.0138 & 0.093 & 0.62 & 10.81 \\
10.5 & 0.0133 & 0.089 & 0.64 & 11.26 \\
11.0 & 0.0130 & 0.086 & 0.65 & 11.65 \\
11.5 & 0.0126 & 0.083 & 0.66 & 12.04 \\
12.0 & 0.0122 & 0.080 & 0.68 & 12.58 \\
12.5 & 0.0119 & 0.077 & 0.69 & 12.95 \\
13.0 & 0.0117 & 0.076 & 0.69 & 13.22 \\
13.5 & 0.0115 & 0.073 & 0.70 & 13.64 \\
\hline
\end{tabular}


Temperature: $-49{ }^{\circ} \mathrm{C}$, Run \#2:

Using General Procedure 2b, benzaldehyde $(67 \mu \mathrm{L}, 0.66 \mathrm{mmol})$ was added to a solution of enolate $4(120 \mu \mathrm{L}, 0.66 \mathrm{mmol})$ and phosphoramide $( \pm)-7 \mathbf{j}(16 \mathrm{mg}, 0.033 \mathrm{mmol})$ in methylene chloride $(2.5 \mathrm{~mL})$ at $-49{ }^{\circ} \mathrm{C}$ as internally monitored. The reaction was monitored every 30 seconds until complete conversion. $\mathrm{k}=77.96 \mathrm{M}^{-2} \min ^{-1}$.

\begin{tabular}{|ccccc|}
\hline Time (min) & Absorbance & [Enolate] & Conversion & 1/[Enolate] \\
\hline \hline 0.0 & 0.0347 & 0.245 & 0.00 & 4.08 \\
0.5 & 0.0305 & 0.215 & 0.12 & 4.65 \\
1.0 & 0.0278 & 0.196 & 0.20 & 5.11 \\
1.5 & 0.0255 & 0.179 & 0.27 & 5.58 \\
2.0 & 0.0240 & 0.168 & 0.31 & 5.94 \\
2.5 & 0.0219 & 0.153 & 0.37 & 6.53 \\
3.0 & 0.0205 & 0.144 & 0.41 & 6.96 \\
3.5 & 0.0193 & 0.135 & 0.45 & 7.42 \\
4.0 & 0.0180 & 0.125 & 0.49 & 8.00 \\
4.5 & 0.0170 & 0.118 & 0.52 & 8.45 \\
5.0 & 0.0161 & 0.112 & 0.54 & 8.95 \\
5.5 & 0.0155 & 0.108 & 0.56 & 9.29 \\
6.0 & 0.0145 & 0.100 & 0.59 & 9.98 \\
6.5 & 0.0139 & 0.096 & 0.61 & 10.43 \\
7.0 & 0.0128 & 0.088 & 0.64 & 11.33 \\
7.5 & 0.0126 & 0.087 & 0.65 & 11.54 \\
8.0 & 0.0119 & 0.082 & 0.67 & 12.20 \\
8.5 & 0.0115 & 0.079 & 0.68 & 12.70 \\
9.0 & 0.0109 & 0.074 & 0.70 & 13.46 \\
9.5 & 0.0103 & 0.071 & 0.71 & 14.16 \\
\hline
\end{tabular}


Temperature: $-49^{\circ} \mathrm{C}$, Run \#3:

Using General Procedure 2b, benzaldehyde $(60 \mu \mathrm{L}, 0.59 \mathrm{mmol})$ was added to a solution of enolate 1 (107 $\mu \mathrm{L}, 0.59 \mathrm{mmol})$ and phosphoramide ( \pm )-7j (15 mg, $0.030 \mathrm{mmol})$ in methylene chloride $(2.2 \mathrm{~mL})$ at $-49{ }^{\circ} \mathrm{C}$ as internally monitored. The reaction was monitored every 30 seconds until complete conversion. $\mathrm{k}=50.93 \mathrm{M}^{-2} \min ^{-1}$.

\begin{tabular}{|c|c|c|c|c|}
\hline Time (min) & Absorbance & [Enolate] & Conversion & 1/[Enolate] \\
\hline 0.0 & 0.0325 & 0.248 & 0.00 & 4.03 \\
\hline 0.5 & 0.0311 & 0.237 & 0.04 & 4.22 \\
\hline 1.0 & 0.0294 & 0.223 & 0.10 & 4.47 \\
\hline 1.5 & 0.0276 & 0.209 & 0.16 & 4.79 \\
\hline 2.0 & 0.0263 & 0.198 & 0.20 & 5.05 \\
\hline 2.5 & 0.0247 & 0.185 & 0.25 & 5.41 \\
\hline 3.0 & 0.0234 & 0.174 & 0.30 & 5.73 \\
\hline 3.5 & 0.0227 & 0.169 & 0.32 & 5.93 \\
\hline 4.0 & 0.0212 & 0.157 & 0.37 & 6.39 \\
\hline 4.5 & 0.0201 & 0.148 & 0.40 & 6.76 \\
\hline 5.0 & 0.0194 & 0.143 & 0.42 & 7.01 \\
\hline 5.5 & 0.0187 & 0.137 & 0.45 & 7.31 \\
\hline 6.0 & 0.0178 & 0.129 & 0.48 & 7.74 \\
\hline 6.5 & 0.0171 & 0.124 & 0.50 & 8.07 \\
\hline 7.0 & 0.0167 & 0.121 & 0.51 & 8.29 \\
\hline 7.5 & 0.0160 & 0.115 & 0.54 & 8.69 \\
\hline 8.0 & 0.0154 & 0.110 & 0.56 & 9.11 \\
\hline 8.5 & 0.0147 & 0.104 & 0.58 & 9.63 \\
\hline 9.0 & 0.0144 & 0.102 & 0.59 & 9.79 \\
\hline 9.5 & 0.0141 & 0.099 & 0.60 & 10.11 \\
\hline 10.0 & 0.0139 & 0.098 & 0.61 & 10.22 \\
\hline 10.5 & 0.0133 & 0.093 & 0.62 & 10.74 \\
\hline 11.0 & 0.0131 & 0.091 & 0.63 & 10.94 \\
\hline 11.5 & 0.0126 & 0.087 & 0.65 & 11.51 \\
\hline 12.0 & 0.0123 & 0.085 & 0.66 & 11.80 \\
\hline 12.5 & 0.0118 & 0.081 & 0.67 & 12.34 \\
\hline 13.0 & 0.0115 & 0.078 & 0.68 & 12.74 \\
\hline 13.5 & 0.0113 & 0.077 & 0.69 & 13.06 \\
\hline 14.0 & 0.0113 & 0.076 & 0.69 & 13.10 \\
\hline 14.5 & 0.0107 & 0.072 & 0.71 & 13.90 \\
\hline
\end{tabular}


Temperature: $-32{ }^{\circ} \mathrm{C}$, Run \#1:

Using General Procedure $2 b$, benzaldehyde $(62 \mu \mathrm{L}, 0.61 \mathrm{mmol})$ was added to a solution of enolate $4(111 \mu \mathrm{L}, 0.61 \mathrm{mmol})$ and phosphoramide $( \pm)-7 \mathbf{j}(15 \mathrm{mg}, 0.031 \mathrm{mmol})$ in methylene chloride $(2.3 \mathrm{~mL})$ at $-32{ }^{\circ} \mathrm{C}$ as internally monitored. The reaction was monitored every 30 seconds until complete conversion. $\mathrm{k}=72.30 \mathrm{M}^{-2} \mathrm{~min}^{-1}$.

\begin{tabular}{|ccccc|}
\hline Time (min) & Absorbance & [Enolate] & Conversion & 1/[Enolate] \\
\hline 0.00 & 0.0325 & 0.276 & 0.00 & 3.62 \\
0.00 & 0.0340 & 0.246 & 0.00 & 4.06 \\
0.50 & 0.0308 & 0.223 & 0.09 & 4.48 \\
1.00 & 0.0281 & 0.203 & 0.17 & 4.92 \\
1.50 & 0.0256 & 0.185 & 0.25 & 5.40 \\
2.00 & 0.0236 & 0.171 & 0.30 & 5.84 \\
2.50 & 0.0222 & 0.161 & 0.35 & 6.23 \\
3.00 & 0.0204 & 0.147 & 0.40 & 6.78 \\
3.50 & 0.0193 & 0.140 & 0.43 & 7.16 \\
4.00 & 0.0182 & 0.131 & 0.47 & 7.61 \\
4.50 & 0.0171 & 0.124 & 0.50 & 8.08 \\
5.00 & 0.0163 & 0.118 & 0.52 & 8.46 \\
5.50 & 0.0155 & 0.112 & 0.55 & 8.94 \\
6.00 & 0.0146 & 0.106 & 0.57 & 9.47 \\
6.50 & 0.0142 & 0.103 & 0.58 & 9.74 \\
7.00 & 0.0133 & 0.096 & 0.61 & 10.42 \\
7.50 & 0.0127 & 0.092 & 0.63 & 10.90 \\
8.00 & 0.0124 & 0.090 & 0.63 & 11.12 \\
8.50 & 0.0119 & 0.086 & 0.65 & 11.68 \\
9.00 & 0.0115 & 0.083 & 0.66 & 12.01 \\
9.50 & 0.0110 & 0.080 & 0.68 & 12.54 \\
10.00 & 0.0105 & 0.076 & 0.69 & 13.12 \\
10.50 & 0.0104 & 0.075 & 0.69 & 13.27 \\
11.00 & 0.0099 & 0.072 & 0.71 & 13.92 \\
\hline
\end{tabular}


Temperature: $-32{ }^{\circ} \mathrm{C}$, Run \#2:

Using General Procedure 2b, benzaldehyde ( $78 \mu \mathrm{L}, 0.77 \mathrm{mmol})$ was added to a solution of enolate $4(140 \mu \mathrm{L}, 0.77 \mathrm{mmol})$ and phosphoramide $( \pm)-7 \mathbf{j}(19 \mathrm{mg}, 0.038 \mathrm{mmol})$ in methylene chloride $(2.9 \mathrm{~mL})$ at $-32{ }^{\circ} \mathrm{C}$ as internally monitored. The reaction was monitored every 30 seconds until complete conversion. $\mathrm{k}=63.05 \mathrm{M}^{-2} \min ^{-1}$.

\begin{tabular}{|ccccc|}
\hline Time (min) & Absorbance & [Enolate] & Conversion & 1/[Enolate] \\
\hline \hline 0.00 & 0.0327 & 0.246 & 0.00 & 4.06 \\
0.50 & 0.0302 & 0.226 & 0.08 & 4.42 \\
1.00 & 0.0277 & 0.208 & 0.16 & 4.82 \\
1.50 & 0.0257 & 0.191 & 0.22 & 5.22 \\
2.00 & 0.0240 & 0.178 & 0.28 & 5.60 \\
2.50 & 0.0224 & 0.166 & 0.33 & 6.02 \\
3.00 & 0.0211 & 0.156 & 0.37 & 6.40 \\
3.50 & 0.0199 & 0.147 & 0.40 & 6.82 \\
4.00 & 0.0191 & 0.141 & 0.43 & 7.12 \\
4.50 & 0.0179 & 0.131 & 0.47 & 7.63 \\
5.00 & 0.0173 & 0.126 & 0.49 & 7.92 \\
5.50 & 0.0165 & 0.120 & 0.51 & 8.30 \\
6.00 & 0.0157 & 0.114 & 0.54 & 8.77 \\
6.50 & 0.0151 & 0.109 & 0.56 & 9.14 \\
7.00 & 0.0143 & 0.103 & 0.58 & 9.69 \\
7.50 & 0.0139 & 0.100 & 0.59 & 10.03 \\
8.00 & 0.0134 & 0.096 & 0.61 & 10.41 \\
8.50 & 0.0128 & 0.091 & 0.63 & 10.93 \\
9.00 & 0.0123 & 0.088 & 0.64 & 11.43 \\
9.50 & 0.0123 & 0.087 & 0.65 & 11.44 \\
10.00 & 0.0118 & 0.083 & 0.66 & 12.00 \\
10.50 & 0.0112 & 0.079 & 0.68 & 12.71 \\
11.00 & 0.0110 & 0.078 & 0.69 & 12.90 \\
11.50 & 0.0107 & 0.075 & 0.69 & 13.28 \\
12.00 & 0.0106 & 0.074 & 0.70 & 13.54 \\
12.50 & 0.0102 & 0.071 & 0.71 & 14.06 \\
\hline
\end{tabular}


Temperature: $-32{ }^{\circ} \mathrm{C}$, Run \#3:

Using General Procedure $2 \mathrm{~b}$, benzaldehyde $(58 \mu \mathrm{L}, 0.57 \mathrm{mmol})$ was added to a solution of enolate $4(103 \mu \mathrm{L}, 0.57 \mathrm{mmol})$ and phosphoramide $( \pm)-7 \mathbf{j}(14 \mathrm{mg}, 0.029 \mathrm{mmol})$ in methylene chloride $(2.1 \mathrm{~mL})$ at $-32{ }^{\circ} \mathrm{C}$ as internally monitored. The reaction was monitored every 30 seconds until complete conversion. $\mathrm{k}=61.46 \mathrm{M}^{-2} \mathrm{~min}^{-1}$.

\begin{tabular}{|ccccc|}
\hline Time (min) & Absorbance & [Enolate] & Conversion & 1/[Enolate] \\
\hline \hline 0.00 & 0.0319 & 0.250 & 0.00 & 4.00 \\
0.50 & 0.0292 & 0.228 & 0.09 & 4.38 \\
1.00 & 0.0270 & 0.210 & 0.16 & 4.76 \\
1.50 & 0.0250 & 0.194 & 0.22 & 5.16 \\
2.00 & 0.0232 & 0.180 & 0.28 & 5.57 \\
2.50 & 0.0218 & 0.169 & 0.33 & 5.93 \\
3.00 & 0.0206 & 0.159 & 0.37 & 6.31 \\
3.50 & 0.0195 & 0.150 & 0.40 & 6.68 \\
4.00 & 0.0183 & 0.140 & 0.44 & 7.12 \\
4.50 & 0.0174 & 0.133 & 0.47 & 7.50 \\
5.00 & 0.0168 & 0.128 & 0.49 & 7.79 \\
5.50 & 0.0159 & 0.121 & 0.52 & 8.27 \\
6.00 & 0.0149 & 0.113 & 0.55 & 8.83 \\
6.50 & 0.0143 & 0.108 & 0.57 & 9.25 \\
7.00 & 0.0137 & 0.103 & 0.59 & 9.71 \\
7.50 & 0.0131 & 0.098 & 0.61 & 10.17 \\
8.00 & 0.0122 & 0.091 & 0.63 & 10.96 \\
8.50 & 0.0118 & 0.088 & 0.65 & 11.36 \\
9.00 & 0.0115 & 0.085 & 0.66 & 11.75 \\
9.50 & 0.0113 & 0.084 & 0.66 & 11.91 \\
10.00 & 0.0108 & 0.080 & 0.68 & 12.57 \\
10.50 & 0.0103 & 0.076 & 0.70 & 13.15 \\
\hline
\end{tabular}


Temperature: $-26^{\circ} \mathrm{C}$, Run \#1:

Using General Procedure $2 \mathrm{~b}$, benzaldehyde $(63 \mu \mathrm{L}, 0.61 \mathrm{mmol})$ was added to a solution of enolate $4(113 \mu \mathrm{L}, 0.62 \mathrm{mmol})$ and phosphoramide $( \pm)-7 \mathbf{j}(15.3 \mathrm{mg}, 0.031 \mathrm{mmol})$ in methylene chloride $(2.3 \mathrm{~mL})$ at $-26{ }^{\circ} \mathrm{C}$ as internally monitored. The reaction was monitored every 30 seconds until complete conversion. $\mathrm{k}=61.77 \mathrm{M}^{-2} \min ^{-1}$.

\begin{tabular}{|ccccc|}
\hline Time (min) & Absorbance & [Enolate] & Conversion & 1/[Enolate] \\
\hline \hline 0.0 & 0.0300 & 0.250 & 0.00 & 4.00 \\
0.5 & 0.0281 & 0.234 & 0.07 & 4.28 \\
1.0 & 0.0266 & 0.220 & 0.12 & 4.54 \\
1.5 & 0.0246 & 0.203 & 0.19 & 4.93 \\
2.0 & 0.0227 & 0.186 & 0.26 & 5.37 \\
2.5 & 0.0218 & 0.178 & 0.29 & 5.62 \\
3.0 & 0.0202 & 0.164 & 0.34 & 6.09 \\
3.5 & 0.0192 & 0.155 & 0.38 & 6.44 \\
4.0 & 0.0179 & 0.144 & 0.43 & 6.96 \\
4.5 & 0.0171 & 0.137 & 0.45 & 7.32 \\
5.0 & 0.0164 & 0.130 & 0.48 & 7.66 \\
5.5 & 0.0155 & 0.122 & 0.51 & 8.17 \\
6.0 & 0.0149 & 0.117 & 0.53 & 8.55 \\
6.5 & 0.0144 & 0.113 & 0.55 & 8.88 \\
7.0 & 0.0136 & 0.106 & 0.58 & 9.47 \\
7.5 & 0.0132 & 0.102 & 0.59 & 9.80 \\
8.0 & 0.0125 & 0.097 & 0.61 & 10.36 \\
8.5 & 0.0123 & 0.094 & 0.62 & 10.63 \\
9.0 & 0.0117 & 0.089 & 0.64 & 11.18 \\
9.5 & 0.0115 & 0.087 & 0.65 & 11.49 \\
10.0 & 0.0110 & 0.083 & 0.67 & 12.11 \\
10.5 & 0.0106 & 0.079 & 0.68 & 12.65 \\
11.0 & 0.0105 & 0.078 & 0.69 & 12.80 \\
11.5 & 0.0102 & 0.076 & 0.70 & 13.16 \\
12.0 & 0.0101 & 0.075 & 0.70 & 13.27 \\
12.5 & 0.0097 & 0.071 & 0.72 & 14.06 \\
\hline
\end{tabular}


Temperature: $-26^{\circ} \mathrm{C}$, Run \#2:

Using General Procedure $2 b$, benzaldehyde $(64 \mu \mathrm{L}, 0.63 \mathrm{mmol})$ was added to a solution of enolate $4(115 \mu \mathrm{L}, 0.63 \mathrm{mmol})$ and phosphoramide $( \pm)-7 \mathbf{j}(15.6 \mathrm{mg}, 0.032 \mathrm{mmol})$ in methylene chloride $(2.3 \mathrm{~mL})$ at $-26^{\circ} \mathrm{C}$ as internally monitored. The reaction was monitored every 30 seconds until complete conversion. $\mathrm{k}=59.54 \mathrm{M}^{-2} \min ^{-1}$.

\begin{tabular}{|ccccc|}
\hline Time (min) & Absorbance & [Enolate] & Conversion & 1/[Enolate] \\
\hline \hline 0.0 & 0.0353 & 0.254 & 0.00 & 3.93 \\
0.5 & 0.0323 & 0.232 & 0.09 & 4.31 \\
1.0 & 0.0299 & 0.214 & 0.16 & 4.68 \\
1.5 & 0.0283 & 0.202 & 0.21 & 4.96 \\
2.0 & 0.0267 & 0.190 & 0.25 & 5.26 \\
2.5 & 0.0242 & 0.171 & 0.33 & 5.85 \\
3.0 & 0.0228 & 0.161 & 0.37 & 6.21 \\
3.5 & 0.0233 & 0.164 & 0.35 & 6.09 \\
4.0 & 0.0208 & 0.146 & 0.43 & 6.85 \\
4.5 & 0.0196 & 0.137 & 0.46 & 7.31 \\
5.0 & 0.0190 & 0.132 & 0.48 & 7.57 \\
5.5 & 0.0175 & 0.121 & 0.52 & 8.25 \\
6.0 & 0.0170 & 0.118 & 0.54 & 8.49 \\
6.5 & 0.0166 & 0.114 & 0.55 & 8.75 \\
7.0 & 0.0158 & 0.109 & 0.57 & 9.21 \\
7.5 & 0.0158 & 0.108 & 0.57 & 9.25 \\
8.0 & 0.0151 & 0.103 & 0.60 & 9.72 \\
8.5 & 0.0146 & 0.100 & 0.61 & 10.02 \\
9.0 & 0.0140 & 0.095 & 0.63 & 10.49 \\
9.5 & 0.0135 & 0.091 & 0.64 & 10.94 \\
10.0 & 0.0137 & 0.093 & 0.64 & 10.80 \\
10.5 & 0.0126 & 0.085 & 0.67 & 11.79 \\
11.0 & 0.0126 & 0.085 & 0.67 & 11.80 \\
11.5 & 0.0120 & 0.080 & 0.68 & 12.44 \\
12.0 & 0.0122 & 0.081 & 0.68 & 12.28 \\
12.5 & 0.0115 & 0.076 & 0.70 & 13.09 \\
13.0 & 0.0114 & 0.075 & 0.70 & 13.25 \\
13.5 & 0.0113 & 0.075 & 0.71 & 13.34 \\
\hline & & & & \\
\hline
\end{tabular}


Temperature: $-26^{\circ} \mathrm{C}$, Run \#3:

Using General Procedure $2 \mathrm{~b}$, benzaldehyde $(63 \mu \mathrm{L}, 0.61 \mathrm{mmol})$ was added to a solution of enolate $4(113 \mu \mathrm{L}, 0.62 \mathrm{mmol})$ and phosphoramide $( \pm)-7 \mathbf{j}(15.6 \mathrm{mg}, 0.032 \mathrm{mmol})$ in methylene chloride $(2.3 \mathrm{~mL})$ at $-26{ }^{\circ} \mathrm{C}$ as internally monitored. The reaction was monitored every 30 seconds until complete conversion. $\mathrm{k}=69.57 \mathrm{M}^{-2} \min ^{-1}$.

\begin{tabular}{|ccccc|}
\hline Time (min) & Absorbance & [Enolate] & Conversion & 1/[Enolate] \\
\hline \hline 0.0 & 0.0274 & 0.250 & 0.00 & 4.00 \\
0.5 & 0.0249 & 0.226 & 0.10 & 4.42 \\
1.0 & 0.0233 & 0.211 & 0.16 & 4.74 \\
1.5 & 0.0212 & 0.191 & 0.24 & 5.24 \\
2.0 & 0.0199 & 0.179 & 0.29 & 5.59 \\
2.5 & 0.0187 & 0.167 & 0.33 & 5.98 \\
3.0 & 0.0175 & 0.155 & 0.38 & 6.44 \\
3.5 & 0.0165 & 0.146 & 0.41 & 6.83 \\
4.0 & 0.0156 & 0.137 & 0.45 & 7.28 \\
4.5 & 0.0144 & 0.126 & 0.50 & 7.92 \\
5.0 & 0.0136 & 0.118 & 0.53 & 8.45 \\
5.5 & 0.0134 & 0.116 & 0.53 & 8.59 \\
6.0 & 0.0126 & 0.109 & 0.56 & 9.14 \\
6.5 & 0.0119 & 0.102 & 0.59 & 9.80 \\
7.0 & 0.0117 & 0.100 & 0.60 & 9.99 \\
7.5 & 0.0112 & 0.095 & 0.62 & 10.49 \\
8.0 & 0.0107 & 0.091 & 0.64 & 11.01 \\
8.5 & 0.0101 & 0.086 & 0.66 & 11.67 \\
9.0 & 0.0098 & 0.083 & 0.67 & 12.08 \\
9.5 & 0.0095 & 0.079 & 0.68 & 12.60 \\
10.0 & 0.0090 & 0.075 & 0.70 & 13.31 \\
10.5 & 0.0087 & 0.072 & 0.71 & 13.92 \\
\hline
\end{tabular}


Temperature: $-10{ }^{\circ} \mathrm{C}$, Run \#1:

Using General Procedure $2 \mathrm{~b}$, benzaldehyde $(63 \mu \mathrm{L}, 0.61 \mathrm{mmol})$ was added to a solution of enolate $4(112 \mu \mathrm{L}, 0.61 \mathrm{mmol})$ and phosphoramide $( \pm)-7 \mathbf{j}(15.2 \mathrm{mg}, 0.031 \mathrm{mmol})$ in methylene chloride $(2.3 \mathrm{~mL})$ at $-10^{\circ} \mathrm{C}$ as internally monitored. The reaction was monitored every 30 seconds until complete conversion. $\mathrm{k}=73.48 \mathrm{M}^{-2} \min ^{-1}$.

\begin{tabular}{|ccccc|}
\hline Time $(\mathbf{m i n})$ & Absorbance & [Enolate] & Conversion & 1/[Enolate] \\
\hline \hline 0.0 & 0.0304 & 0.248 & 0.00 & 4.03 \\
0.5 & 0.0278 & 0.225 & 0.09 & 4.44 \\
1.0 & 0.0256 & 0.207 & 0.17 & 4.83 \\
1.5 & 0.0235 & 0.189 & 0.24 & 5.28 \\
2.0 & 0.0215 & 0.173 & 0.30 & 5.79 \\
2.5 & 0.0204 & 0.163 & 0.34 & 6.12 \\
3.0 & 0.0188 & 0.150 & 0.40 & 6.67 \\
3.5 & 0.0178 & 0.141 & 0.43 & 7.09 \\
4.0 & 0.0166 & 0.132 & 0.47 & 7.60 \\
4.5 & 0.0157 & 0.123 & 0.50 & 8.10 \\
5.0 & 0.0148 & 0.116 & 0.53 & 8.60 \\
5.5 & 0.0139 & 0.108 & 0.56 & 9.24 \\
6.0 & 0.0137 & 0.107 & 0.57 & 9.37 \\
6.5 & 0.0127 & 0.098 & 0.60 & 10.18 \\
7.0 & 0.0123 & 0.095 & 0.62 & 10.49 \\
7.5 & 0.0116 & 0.089 & 0.64 & 11.24 \\
8.0 & 0.0113 & 0.087 & 0.65 & 11.54 \\
8.5 & 0.0105 & 0.080 & 0.68 & 12.53 \\
9.0 & 0.0102 & 0.078 & 0.69 & 12.89 \\
9.5 & 0.0100 & 0.075 & 0.70 & 13.28 \\
10.0 & 0.0095 & 0.071 & 0.71 & 14.09 \\
\hline
\end{tabular}


Temperature: $-10{ }^{\circ} \mathrm{C}$, Run \#2:

Using General Procedure $2 \mathrm{~b}$, benzaldehyde $(54 \mu \mathrm{L}, 0.54 \mathrm{mmol})$ was added to a solution of enolate $4(98 \mu \mathrm{L}, 0.54 \mathrm{mmol})$ and phosphoramide $( \pm)-7 \mathbf{j}(13.2 \mathrm{mg}, 0.027 \mathrm{mmol})$ in methylene chloride $(2.0 \mathrm{~mL})$ at $-10{ }^{\circ} \mathrm{C}$ as internally monitored. The reaction was monitored every 30 seconds until complete conversion. $\mathrm{k}=71.19 \mathrm{M}^{-2} \min ^{-1}$.

\begin{tabular}{|ccccc|}
\hline Time (min) & Absorbance & [Enolate] & Conversion & 1/[Enolate] \\
\hline 0.0 & 0.0302 & 0.250 & 0.00 & 4.00 \\
0.5 & 0.0274 & 0.225 & 0.10 & 4.44 \\
1.0 & 0.0253 & 0.207 & 0.17 & 4.83 \\
1.5 & 0.0237 & 0.192 & 0.23 & 5.20 \\
2.0 & 0.0213 & 0.172 & 0.31 & 5.83 \\
2.5 & 0.0202 & 0.161 & 0.35 & 6.19 \\
3.0 & 0.0190 & 0.151 & 0.39 & 6.60 \\
3.5 & 0.0180 & 0.142 & 0.43 & 7.03 \\
4.0 & 0.0169 & 0.133 & 0.47 & 7.52 \\
4.5 & 0.0162 & 0.127 & 0.49 & 7.90 \\
5.0 & 0.0152 & 0.118 & 0.53 & 8.47 \\
5.5 & 0.0146 & 0.113 & 0.55 & 8.88 \\
6.0 & 0.0139 & 0.106 & 0.57 & 9.40 \\
6.5 & 0.0134 & 0.103 & 0.59 & 9.76 \\
7.0 & 0.0126 & 0.096 & 0.62 & 10.46 \\
7.5 & 0.0124 & 0.094 & 0.62 & 10.65 \\
8.0 & 0.0119 & 0.089 & 0.64 & 11.22 \\
8.5 & 0.0113 & 0.084 & 0.66 & 11.88 \\
9.0 & 0.0110 & 0.081 & 0.67 & 12.32 \\
9.5 & 0.0106 & 0.078 & 0.69 & 12.81 \\
10.0 & 0.0101 & 0.073 & 0.71 & 13.65 \\
\hline
\end{tabular}


Temperature: $-10{ }^{\circ} \mathrm{C}$, Run \#3:

Using General Procedure $2 \mathrm{~b}$, benzaldehyde $(62 \mu \mathrm{L}, 0.61 \mathrm{mmol})$ was added to a solution of enolate $4(111 \mu \mathrm{L}, 0.61 \mathrm{mmol})$ and phosphoramide $( \pm)-7 \mathbf{j}(15 \mathrm{mg}, 0.030 \mathrm{mmol})$ in methylene chloride $(2.0 \mathrm{~mL})$ at $-10{ }^{\circ} \mathrm{C}$ as internally monitored. The reaction was monitored every 30 seconds until complete conversion. $\mathrm{k}=70.97 \mathrm{M}^{-2} \min ^{-1}$.

\begin{tabular}{|ccccc|}
\hline Time (min) & Absorbance & [Enolate] & Conversion & 1/[Enolate] \\
\hline \hline 0.0 & 0.0321 & 0.246 & 0.00 & 4.06 \\
0.5 & 0.0293 & 0.224 & 0.09 & 4.46 \\
1.0 & 0.0270 & 0.206 & 0.16 & 4.86 \\
1.5 & 0.0245 & 0.186 & 0.24 & 5.38 \\
2.0 & 0.0232 & 0.175 & 0.29 & 5.70 \\
2.5 & 0.0217 & 0.163 & 0.34 & 6.13 \\
3.0 & 0.0203 & 0.152 & 0.38 & 6.57 \\
3.5 & 0.0189 & 0.141 & 0.43 & 7.07 \\
4.0 & 0.0179 & 0.133 & 0.46 & 7.51 \\
4.5 & 0.0171 & 0.126 & 0.49 & 7.91 \\
5.0 & 0.0161 & 0.118 & 0.52 & 8.44 \\
5.5 & 0.0157 & 0.116 & 0.53 & 8.63 \\
6.0 & 0.0149 & 0.109 & 0.56 & 9.17 \\
6.5 & 0.0144 & 0.105 & 0.57 & 9.54 \\
7.0 & 0.0136 & 0.099 & 0.60 & 10.13 \\
7.5 & 0.0128 & 0.093 & 0.62 & 10.79 \\
8.0 & 0.0126 & 0.091 & 0.63 & 10.96 \\
8.5 & 0.0120 & 0.086 & 0.65 & 11.60 \\
9.0 & 0.0118 & 0.085 & 0.66 & 11.81 \\
9.5 & 0.0112 & 0.080 & 0.68 & 12.52 \\
10.0 & 0.0107 & 0.076 & 0.69 & 13.21 \\
11.0 & 0.0101 & 0.075 & 0.71 & 14.10 \\
\hline
\end{tabular}

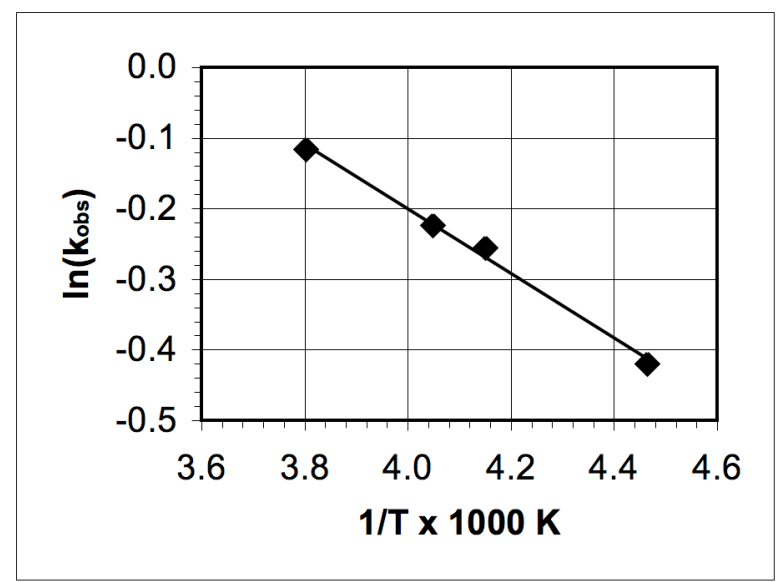

Figure IV. Arrhenius Plot. The graph depicts a linear fit, $f(x)=m x+b\left(m=-0.46, R^{2}=0.99\right)$. 


\section{Raw Data. ReactIR Loading Studies on Addition of 4 to 5a Catalyzed by ( \pm )-7j,}

Temperature: $-34{ }^{\circ} \mathrm{C}, 1 \mathrm{~mol} \%$ :

Using General Procedure 2b, benzaldehyde (66 $\mu \mathrm{L}, 0.65 \mathrm{mmol})$ was added to a solution of enolate $4(118 \mu \mathrm{L}, 0.65 \mathrm{mmol})$ and phosphoramide $( \pm)-7 \mathbf{j}(3.2 \mathrm{mg}, 0.007 \mathrm{mmol})$ in methylene chloride $(2.4 \mathrm{~mL})$ at $-34{ }^{\circ} \mathrm{C}$ as internally monitored. The reaction was monitored every 30 seconds until complete conversion. $\mathrm{k}_{\mathrm{obs}}=0.513 \mathrm{M}^{-1} \mathrm{~min}^{-1}$.

\begin{tabular}{|c|c|c|c|c|}
\hline Time (min) & Absorbance & [Enolate] & Conversion & 1/[Enolate] \\
\hline 0.0 & 0.0344 & 0.250 & 0.00 & 3.99 \\
\hline 0.5 & 0.0326 & 0.238 & 0.05 & 4.21 \\
\hline 1.0 & 0.0309 & 0.225 & 0.10 & 4.44 \\
\hline 1.5 & 0.0295 & 0.215 & 0.14 & 4.65 \\
\hline 2.0 & 0.0276 & 0.201 & 0.20 & 4.98 \\
\hline 2.5 & 0.0265 & 0.193 & 0.23 & 5.17 \\
\hline 3.0 & 0.0253 & 0.184 & 0.26 & 5.43 \\
\hline 3.5 & 0.0240 & 0.175 & 0.30 & 5.73 \\
\hline 4.0 & 0.0234 & 0.170 & 0.32 & 5.87 \\
\hline 4.5 & 0.0221 & 0.161 & 0.36 & 6.22 \\
\hline 5.0 & 0.0210 & 0.153 & 0.39 & 6.53 \\
\hline 5.5 & 0.0206 & 0.150 & 0.40 & 6.68 \\
\hline 6.0 & 0.0197 & 0.144 & 0.43 & 6.95 \\
\hline 6.5 & 0.0186 & 0.135 & 0.46 & 7.38 \\
\hline 7.0 & 0.0184 & 0.134 & 0.46 & 7.44 \\
\hline 7.5 & 0.0177 & 0.129 & 0.48 & 7.74 \\
\hline 8.0 & 0.0170 & 0.124 & 0.51 & 8.07 \\
\hline 8.5 & 0.0167 & 0.122 & 0.51 & 8.23 \\
\hline 9.0 & 0.0159 & 0.116 & 0.54 & 8.64 \\
\hline 9.5 & 0.0156 & 0.113 & 0.55 & 8.82 \\
\hline 10.0 & 0.0152 & 0.111 & 0.56 & 9.00 \\
\hline 10.5 & 0.0149 & 0.109 & 0.57 & 9.20 \\
\hline 11.0 & 0.0143 & 0.104 & 0.58 & 9.61 \\
\hline 11.5 & 0.0140 & 0.102 & 0.59 & 9.81 \\
\hline 12.0 & 0.0137 & 0.100 & 0.60 & 9.98 \\
\hline 12.5 & 0.0132 & 0.096 & 0.62 & 10.40 \\
\hline 13.0 & 0.0125 & 0.091 & 0.64 & 10.97 \\
\hline 13.5 & 0.0128 & 0.093 & 0.63 & 10.71 \\
\hline 14.0 & 0.0122 & 0.089 & 0.64 & 11.24 \\
\hline 14.5 & 0.0121 & 0.088 & 0.65 & 11.31 \\
\hline 15.0 & 0.0118 & 0.086 & 0.66 & 11.67 \\
\hline 15.5 & 0.0115 & 0.083 & 0.67 & 11.98 \\
\hline 16.0 & 0.0113 & 0.082 & 0.67 & 12.15 \\
\hline 16.5 & 0.0110 & 0.080 & 0.68 & 12.50 \\
\hline 17.0 & 0.0109 & 0.079 & 0.68 & 12.60 \\
\hline 17.5 & 0.0108 & 0.079 & 0.68 & 12.67 \\
\hline
\end{tabular}




\begin{tabular}{|lllll|}
18.0 & 0.0102 & 0.074 & 0.70 & 13.44 \\
18.5 & 0.0104 & 0.076 & 0.70 & 13.15 \\
19.0 & 0.0102 & 0.074 & 0.70 & 13.49 \\
\hline
\end{tabular}

Temperature: $-34{ }^{\circ} \mathrm{C}, 10 \mathrm{~mol} \%$ :

Using General Procedure $2 \mathrm{~b}$, benzaldehyde $(66 \mu \mathrm{L}, 0.65 \mathrm{mmol}$ ) was added to a solution of enolate $4(119 \mu \mathrm{L}, 0.65 \mathrm{mmol})$ and phosphoramide $( \pm)-7 \mathbf{j}(32 \mathrm{mg}, 0.065 \mathrm{mmol})$ in methylene chloride $(2.4 \mathrm{~mL})$ at $-34{ }^{\circ} \mathrm{C}$ as internally monitored. The reaction was monitored every 30 seconds until complete conversion. $\mathrm{k}_{\mathrm{obs}}=0.778 \mathrm{M}^{-1} \mathrm{~min}^{-1}$.

\begin{tabular}{|ccccc|}
\hline Time (min) & Absorbance & [Enolate] & Conversion & 1/[Enolate] \\
\hline 0.0 & 0.0342 & 0.252 & 0.00 & 3.96 \\
0.5 & 0.0313 & 0.230 & 0.09 & 4.34 \\
1.0 & 0.0289 & 0.212 & 0.16 & 4.71 \\
1.5 & 0.0267 & 0.196 & 0.23 & 5.11 \\
2.0 & 0.0249 & 0.182 & 0.28 & 5.50 \\
2.5 & 0.0235 & 0.172 & 0.32 & 5.83 \\
3.0 & 0.0217 & 0.158 & 0.38 & 6.34 \\
3.5 & 0.0207 & 0.150 & 0.40 & 6.66 \\
4.0 & 0.0196 & 0.142 & 0.44 & 7.07 \\
4.5 & 0.0187 & 0.135 & 0.46 & 7.40 \\
5.0 & 0.0178 & 0.128 & 0.49 & 7.82 \\
5.5 & 0.0170 & 0.122 & 0.52 & 8.18 \\
6.0 & 0.0161 & 0.115 & 0.54 & 8.68 \\
6.5 & 0.0152 & 0.109 & 0.57 & 9.19 \\
7.0 & 0.0148 & 0.106 & 0.58 & 9.48 \\
7.5 & 0.0141 & 0.100 & 0.60 & 10.01 \\
8.0 & 0.0134 & 0.095 & 0.62 & 10.53 \\
8.5 & 0.0130 & 0.092 & 0.64 & 10.88 \\
9.0 & 0.0127 & 0.089 & 0.65 & 11.20 \\
9.5 & 0.0119 & 0.083 & 0.67 & 12.00 \\
10.0 & 0.0116 & 0.081 & 0.68 & 12.32 \\
10.5 & 0.0115 & 0.080 & 0.68 & 12.48 \\
11.0 & 0.0112 & 0.078 & 0.69 & 12.85 \\
11.5 & 0.0107 & 0.075 & 0.70 & 13.41 \\
\hline
\end{tabular}


Temperature: $-34^{\circ} \mathrm{C}, 20 \mathrm{~mol} \%$ :

Using General Procedure $2 \mathrm{~b}$, benzaldehyde ( $67 \mu \mathrm{L}, 0.66 \mathrm{mmol})$ was added to a solution of enolate $4(120 \mu \mathrm{L}, 0.66 \mathrm{mmol})$ and phosphoramide $( \pm)-7 \mathbf{j}(65 \mathrm{mg}, 0.131 \mathrm{mmol})$ in methylene chloride $(2.4 \mathrm{~mL})$ at $-34{ }^{\circ} \mathrm{C}$ as internally monitored. The reaction was monitored every 30 seconds until complete conversion. $\mathrm{k}_{\mathrm{obs}}=1.048 \mathrm{M}^{-1} \mathrm{~min}^{-1}$.

\begin{tabular}{|ccccc|}
\hline Time (min) & Absorbance & [Enolate] & Conversion & 1/[Enolate] \\
\hline 0.0 & 0.0344 & 0.254 & 0.00 & 3.93 \\
0.3 & 0.0318 & 0.234 & 0.08 & 4.27 \\
0.7 & 0.0293 & 0.215 & 0.16 & 4.66 \\
1.0 & 0.0274 & 0.200 & 0.21 & 5.00 \\
1.3 & 0.0258 & 0.187 & 0.26 & 5.34 \\
1.7 & 0.0241 & 0.175 & 0.31 & 5.73 \\
2.0 & 0.0231 & 0.166 & 0.35 & 6.01 \\
2.3 & 0.0219 & 0.157 & 0.38 & 6.36 \\
2.7 & 0.0211 & 0.151 & 0.41 & 6.64 \\
3.0 & 0.0198 & 0.140 & 0.45 & 7.13 \\
3.3 & 0.0190 & 0.134 & 0.47 & 7.44 \\
3.7 & 0.0181 & 0.128 & 0.50 & 7.84 \\
4.0 & 0.0177 & 0.124 & 0.51 & 8.03 \\
4.3 & 0.0168 & 0.117 & 0.54 & 8.53 \\
4.7 & 0.0163 & 0.113 & 0.55 & 8.83 \\
5.0 & 0.0155 & 0.107 & 0.58 & 9.35 \\
5.3 & 0.0153 & 0.106 & 0.58 & 9.47 \\
5.7 & 0.0144 & 0.099 & 0.61 & 10.13 \\
6.0 & 0.0142 & 0.097 & 0.62 & 10.28 \\
6.3 & 0.0142 & 0.097 & 0.62 & 10.29 \\
6.7 & 0.0135 & 0.092 & 0.64 & 10.92 \\
7.0 & 0.0130 & 0.088 & 0.65 & 11.38 \\
7.3 & 0.0130 & 0.088 & 0.66 & 11.40 \\
7.7 & 0.0129 & 0.087 & 0.66 & 11.56 \\
8.0 & 0.0121 & 0.081 & 0.68 & 12.35 \\
8.3 & 0.0119 & 0.079 & 0.69 & 12.72 \\
8.7 & 0.0116 & 0.076 & 0.70 & 13.10 \\
9.0 & 0.0115 & 0.076 & 0.70 & 13.13 \\
\hline
\end{tabular}


Temperature: $-34{ }^{\circ} \mathrm{C}, 30 \mathrm{~mol} \%$ :

Using General Procedure $2 b$, benzaldehyde $(63 \mu \mathrm{L}, 0.62 \mathrm{mmol})$ was added to a solution of enolate $4(112 \mu \mathrm{L}, 0.61 \mathrm{mmol})$ and phosphoramide $( \pm)-7 \mathbf{j}(88 \mathrm{mg}, 0.179 \mathrm{mmol})$ in methylene chloride $(2.3 \mathrm{~mL})$ at $-34{ }^{\circ} \mathrm{C}$ as internally monitored. The reaction was monitored every 20 seconds until complete conversion. $\mathrm{k}_{\mathrm{obs}}=1.305 \mathrm{M}^{-1} \mathrm{~min}^{-1}$.

\begin{tabular}{|ccccc|}
\hline Time (min) & Absorbance & [Enolate] & Conversion & 1/[Enolate] \\
\hline 0.0 & 0.0344 & 0.254 & 0.00 & 3.93 \\
0.3 & 0.0318 & 0.234 & 0.08 & 4.27 \\
0.7 & 0.0293 & 0.215 & 0.16 & 4.66 \\
1.0 & 0.0274 & 0.200 & 0.21 & 5.00 \\
1.3 & 0.0258 & 0.187 & 0.26 & 5.34 \\
1.7 & 0.0241 & 0.175 & 0.31 & 5.73 \\
2.0 & 0.0231 & 0.166 & 0.35 & 6.01 \\
2.3 & 0.0219 & 0.157 & 0.38 & 6.36 \\
2.7 & 0.0211 & 0.151 & 0.41 & 6.64 \\
3.0 & 0.0198 & 0.140 & 0.45 & 7.13 \\
3.3 & 0.0190 & 0.134 & 0.47 & 7.44 \\
3.7 & 0.0181 & 0.128 & 0.50 & 7.84 \\
4.0 & 0.0177 & 0.124 & 0.51 & 8.03 \\
4.3 & 0.0168 & 0.117 & 0.54 & 8.53 \\
4.7 & 0.0163 & 0.113 & 0.55 & 8.83 \\
5.0 & 0.0155 & 0.107 & 0.58 & 9.35 \\
5.3 & 0.0153 & 0.106 & 0.58 & 9.47 \\
5.7 & 0.0144 & 0.099 & 0.61 & 10.13 \\
6.0 & 0.0142 & 0.097 & 0.62 & 10.28 \\
6.3 & 0.0142 & 0.097 & 0.62 & 10.29 \\
6.7 & 0.0135 & 0.092 & 0.64 & 10.92 \\
7.0 & 0.0130 & 0.088 & 0.65 & 11.38 \\
7.3 & 0.0130 & 0.088 & 0.66 & 11.40 \\
7.7 & 0.0129 & 0.087 & 0.66 & 11.56 \\
8.0 & 0.0121 & 0.081 & 0.68 & 12.35 \\
8.3 & 0.0119 & 0.079 & 0.69 & 12.72 \\
8.7 & 0.0116 & 0.076 & 0.70 & 13.10 \\
9.0 & 0.0115 & 0.076 & 0.70 & 13.13 \\
\hline
\end{tabular}




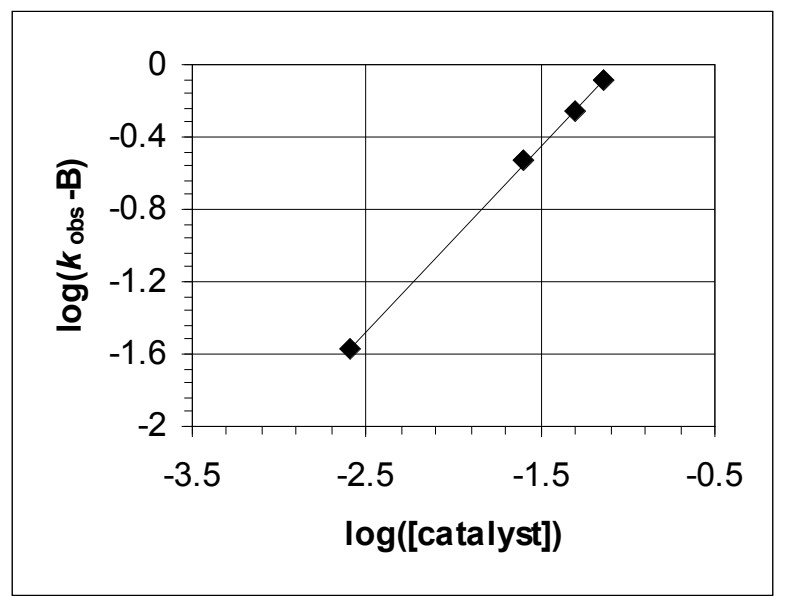

Figure V. Plot of $\log \left(k_{\mathrm{obs}}-\mathrm{B}\right)$ versus $\log ([$ catalyst] $)$ for the addition of $\mathbf{4}$ to $\mathbf{5 a}$ catalyzed by $\mathbf{7} \mathbf{j}$ at $\mathrm{T}=-35^{\circ} \mathrm{C}$. The graph depicts the linear fit to $f(x)=m x+b\left(m=1.014, R^{2}=1.000\right)$. 


\section{Raw Data. RINMR kinetics of Addition of 4 to 5a Catalyzed by ( \pm )-7j}

Temperature: $-27^{\circ} \mathrm{C}$ :

Using General Procedure 3a, a solution of benzaldehyde in chloroform (85 $\mu \mathrm{L}, 0.35$ mmol) was injected into an NMR tube containing enolate $4(65 \mu \mathrm{L}, 0.65 \mathrm{mmol})$ and phosphoramide $( \pm)-7 \mathbf{j}(8.6 \mathrm{mg}, 0.018 \mathrm{mmol})$ in methylene chloride- $d_{2}(0.6 \mathrm{~mL})$ at $-27{ }^{\circ} \mathrm{C}$ as internally monitored. The reaction was monitored every 37 seconds until complete conversion. $\mathrm{k}=0.4373 \mathrm{M}^{-2} \mathrm{~s}^{-1}$.

\begin{tabular}{|ccccc|}
\hline Time (s) & \% Enolate & \% Aldolate & [Enolate] & 1/[Enolate] \\
\hline \hline 0 & 0.00 & 0.00 & 0.437 & 2.289 \\
11 & 97.17 & 2.83 & 0.425 & 2.355 \\
48 & 84.61 & 15.39 & 0.370 & 2.705 \\
85 & 75.20 & 24.80 & 0.329 & 3.043 \\
122 & 67.69 & 32.31 & 0.296 & 3.381 \\
159 & 60.95 & 39.05 & 0.266 & 3.755 \\
196 & 55.60 & 44.40 & 0.243 & 4.116 \\
233 & 50.65 & 49.35 & 0.221 & 4.519 \\
\hline
\end{tabular}

Temperature: $-37^{\circ} \mathrm{C}$ :

Using General Procedure 3a, a solution of benzaldehyde in chloroform ( $85 \mu \mathrm{L}, 0.35$ mmol) was injected into an NMR tube containing enolate $4(65 \mu \mathrm{L}, 0.65 \mathrm{mmol})$ and phosphoramide, $( \pm)-7 \mathbf{j}(8.6 \mathrm{mg}, 0.018 \mathrm{mmol})$ in methylene chloride- $d_{2}(0.6 \mathrm{~mL})$ at $-37{ }^{\circ} \mathrm{C}$ as internally monitored. The reaction was monitored every 37 seconds until complete conversion. $\mathrm{k}=0.353 \mathrm{M}^{-2} \mathrm{~s}^{-1}$.

\begin{tabular}{|ccccc|}
\hline Time (s) & \% Enolate & \% Aldolate & [Enolate] & 1/[Enolate] \\
\hline \hline 0 & 0.00 & 0.00 & 0.437 & 2.289 \\
11 & 97.59 & 2.41 & 0.426 & 2.345 \\
48 & 86.84 & 13.16 & 0.379 & 2.635 \\
85 & 78.23 & 21.77 & 0.342 & 2.925 \\
122 & 70.82 & 29.18 & 0.309 & 3.232 \\
159 & 64.70 & 35.30 & 0.283 & 3.537 \\
196 & 59.85 & 40.15 & 0.262 & 3.824 \\
233 & 55.83 & 44.17 & 0.244 & 4.099 \\
270 & 52.66 & 47.34 & 0.230 & 4.346 \\
307 & 49.60 & 50.40 & 0.217 & 4.614 \\
\hline
\end{tabular}




\section{Temperature: $-49^{\circ} \mathrm{C}$ :}

Using General Procedure 3a, a solution of benzaldehyde in chloroform (85 $\mu \mathrm{L}, 0.35$ mmol) was injected into an NMR tube containing enolate 4 (65 $\mu \mathrm{L}, 0.65 \mathrm{mmol})$ and phosphoramide $( \pm)-7 \mathbf{j}(8.6 \mathrm{mg}, 0.018 \mathrm{mmol})$ in methylene chloride- $d_{2}(0.6 \mathrm{~mL})$ at $-49{ }^{\circ} \mathrm{C}$ as internally monitored. The reaction was monitored every 37 seconds until complete conversion. $\mathrm{k}=0.300 \mathrm{M}^{-2} \mathrm{~s}^{-1}$.

\begin{tabular}{|ccccc|}
\hline Time (s) & \% Enolate & \% Aldolate & [Enolate] & 1/[Enolate] \\
\hline \hline 0 & 100.00 & 0.00 & 0.437 & 2.289 \\
180 & 92.84 & 7.16 & 0.406 & 2.465 \\
217 & 78.84 & 21.16 & 0.345 & 2.903 \\
254 & 66.58 & 33.42 & 0.291 & 3.437 \\
291 & 57.47 & 42.53 & 0.251 & 3.982 \\
328 & 50.49 & 49.51 & 0.221 & 4.532 \\
365 & 45.32 & 54.68 & 0.198 & 5.050 \\
\hline
\end{tabular}

\section{Temperature: $-58^{\circ} \mathrm{C}$ :}

Using General Procedure 3a, a solution of benzaldehyde in chloroform ( $85 \mu \mathrm{L}, 0.35$ mmol) was injected into an NMR tube containing enolate 4 (65 $\mu \mathrm{L}, 0.65 \mathrm{mmol})$ and phosphoramide $( \pm)-7 \mathbf{j}(8.6 \mathrm{mg}, 0.018 \mathrm{mmol})$ in methylene chloride- $d_{2}(0.6 \mathrm{~mL})$ at $-58{ }^{\circ} \mathrm{C}$ as internally monitored. The reaction was monitored every 67 seconds until complete conversion. $\mathrm{k}=0.247 \mathrm{M}^{-2} \mathrm{~s}^{-1}$.

\begin{tabular}{|ccccc|}
\hline Time (s) & \% Enolate & \% Aldolate & [Enolate] & 1/[Enolate] \\
\hline \hline 0 & 100.00 & 0.00 & 0.437 & 2.289 \\
90 & 83.68 & 16.32 & 0.366 & 2.735 \\
157 & 73.16 & 26.84 & 0.320 & 3.128 \\
224 & 64.85 & 35.15 & 0.283 & 3.529 \\
291 & 58.84 & 41.16 & 0.257 & 3.889 \\
358 & 54.19 & 45.81 & 0.237 & 4.223 \\
425 & 50.35 & 49.65 & 0.220 & 4.546 \\
\hline
\end{tabular}




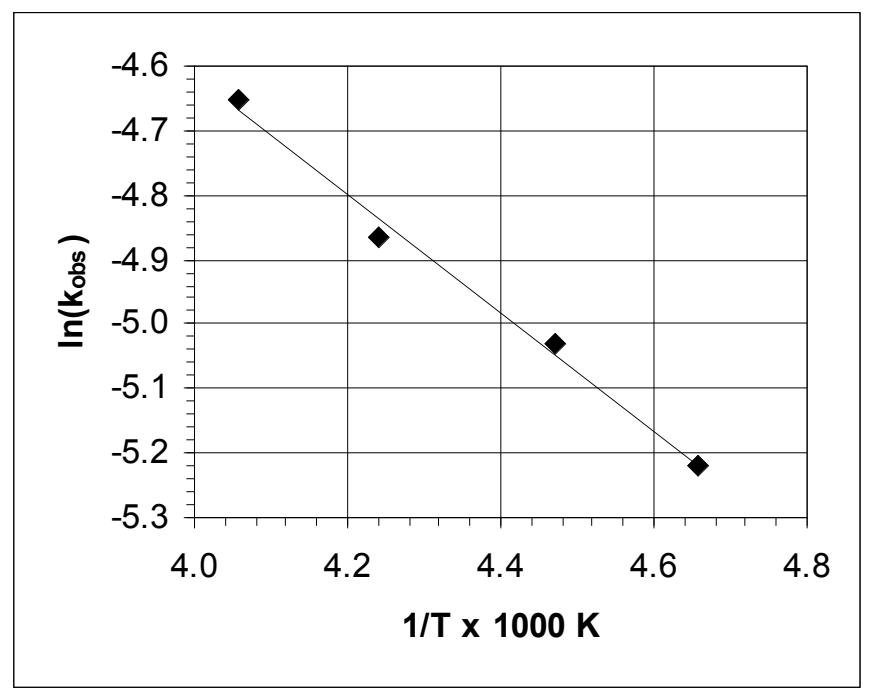

Figure VI. Arrhenius Plot. The graph depicts a linear fit, $f(x)=m x+b\left(m=-0.92, R^{2}=0.99\right)$. 
Raw Data. RINMR Loading Studies on the Addition of 4 to 5 a Catalyzed by ( \pm )-7j.

Temperature: $-53{ }^{\circ} \mathrm{C}, 5 \mathrm{~mol} \%$ :

Using General Procedure 3a, a solution of benzaldehyde in chloroform ( $85 \mu \mathrm{L}, 0.35 \mathrm{mmol}$ ) was injected into an NMR tube containing enolate $4(65 \mu \mathrm{L}, 0.65 \mathrm{mmol})$ and phosphoramide $( \pm)-7 \mathbf{j}$ $(8.6 \mathrm{mg}, 0.018 \mathrm{mmol})$ in methylene chloride- $d_{2}(0.6 \mathrm{~mL})$ at $-53{ }^{\circ} \mathrm{C}$ as internally monitored. The reaction was monitored every 10 seconds until complete conversion. $\mathrm{k}_{\mathrm{obs}}=0.012 \mathrm{M}^{-1} \mathrm{~s}^{-1}$.

\begin{tabular}{|ccccc|}
\hline Time (s) & \% Enolate & \% Aldolate & [Enolate] & 1/[Enolate] \\
\hline 0 & 100.00 & 0.00 & 0.470 & 2.129 \\
4 & - & - & - & - \\
14 & 86.68 & 13.32 & 0.407 & 2.456 \\
24 & 83.62 & 16.38 & 0.393 & 2.545 \\
34 & 80.66 & 19.34 & 0.379 & 2.639 \\
44 & 77.70 & 22.30 & 0.365 & 2.739 \\
54 & 75.11 & 24.89 & 0.353 & 2.834 \\
64 & 72.67 & 27.33 & 0.341 & 2.929 \\
74 & 70.50 & 29.50 & 0.331 & 3.019 \\
84 & 68.46 & 31.54 & 0.322 & 3.109 \\
94 & 66.53 & 33.47 & 0.313 & 3.199 \\
104 & 64.76 & 35.24 & 0.304 & 3.287 \\
114 & 63.02 & 36.98 & 0.296 & 3.378 \\
124 & 61.49 & 38.51 & 0.289 & 3.462 \\
134 & 60.28 & 39.72 & 0.283 & 3.531 \\
\hline
\end{tabular}


Temperature: $-53^{\circ} \mathrm{C}, 3 \mathrm{~mol} \%$ :

Using General Procedure 3a, a solution of benzaldehyde in chloroform ( $85 \mu \mathrm{L}, 0.35 \mathrm{mmol})$ was injected into an NMR tube containing enolate $4(65 \mu \mathrm{L}, 0.65 \mathrm{mmol})$ and phosphoramide $( \pm)-7 \mathbf{j}$ $(5.1 \mathrm{mg}, 0.010 \mathrm{mmol})$ in methylene chloride- $d_{2}(0.6 \mathrm{~mL})$ at $-53{ }^{\circ} \mathrm{C}$ as internally monitored. The reaction was monitored every 10 seconds until complete conversion. $\mathrm{k}_{\mathrm{obs}}=0.011 \mathrm{M}^{-1} \mathrm{~s}^{-1}$.

\begin{tabular}{|ccccc|}
\hline Time (s) & \% Enolate & \% Aldolate & [Enolate] & 1/[Enolate] \\
\hline \hline 0 & 100.00 & 0.00 & 0.470 & 2.129 \\
4 & - & - & - & - \\
14 & 91.65 & 8.35 & 0.431 & 2.323 \\
24 & 88.08 & 11.92 & 0.414 & 2.417 \\
34 & 84.75 & 15.25 & 0.398 & 2.512 \\
44 & 81.54 & 18.46 & 0.383 & 2.611 \\
54 & 76.14 & 23.86 & 0.358 & 2.795 \\
64 & 73.51 & 26.49 & 0.345 & 2.896 \\
74 & 71.22 & 28.78 & 0.335 & 2.989 \\
84 & 68.95 & 31.05 & 0.324 & 3.087 \\
94 & 66.99 & 33.01 & 0.315 & 3.177 \\
104 & 65.08 & 34.92 & 0.306 & 3.270 \\
114 & 63.33 & 36.67 & 0.298 & 3.361 \\
124 & 61.70 & 38.30 & 0.290 & 3.450 \\
134 & 60.26 & 39.74 & 0.283 & 3.532 \\
144 & 58.77 & 41.23 & 0.276 & 3.622 \\
154 & 57.54 & 42.46 & 0.270 & 3.699 \\
164 & 56.30 & 43.70 & 0.264 & 3.781 \\
\hline
\end{tabular}


Temperature: $-53^{\circ} \mathrm{C}, 2 \mathrm{~mol} \%$ :

Using General Procedure 3a, a solution of benzaldehyde in chloroform ( $85 \mu \mathrm{L}, 0.35 \mathrm{mmol})$ was injected into an NMR tube containing enolate $4(65 \mu \mathrm{L}, 0.65 \mathrm{mmol})$ and phosphoramide $( \pm)-7 \mathbf{j}$ (3.4 mg, $0.007 \mathrm{mmol})$ in methylene chloride- $d_{2}(0.6 \mathrm{~mL})$ at $-53{ }^{\circ} \mathrm{C}$ as internally monitored. The reaction was monitored every 10 seconds until complete conversion. $\mathrm{k}_{\mathrm{obs}}=0.009 \mathrm{M}^{-1} \mathrm{~s}^{-1}$.

\begin{tabular}{|ccccc|}
\hline Time (s) & \% Enolate & \% Aldolate & [Enolate] & 1/[Enolate] \\
\hline \hline 0 & 100.00 & 0.00 & 0.470 & 2.129 \\
4 & - & - & - & - \\
14 & 93.08 & 6.92 & 0.437 & 2.287 \\
24 & 89.15 & 10.85 & 0.419 & 2.388 \\
34 & 85.93 & 14.07 & 0.404 & 2.477 \\
44 & 82.78 & 17.22 & 0.389 & 2.571 \\
54 & 79.17 & 20.83 & 0.372 & 2.689 \\
64 & 77.15 & 22.85 & 0.362 & 2.759 \\
74 & 74.59 & 25.41 & 0.350 & 2.854 \\
84 & 72.24 & 27.76 & 0.339 & 2.946 \\
94 & 70.11 & 29.89 & 0.329 & 3.036 \\
104 & 68.10 & 31.90 & 0.320 & 3.126 \\
114 & 66.20 & 33.80 & 0.311 & 3.215 \\
124 & 64.45 & 35.55 & 0.303 & 3.303 \\
134 & 62.86 & 37.14 & 0.295 & 3.386 \\
144 & 61.35 & 38.65 & 0.288 & 3.469 \\
154 & 59.97 & 40.03 & 0.282 & 3.550 \\
164 & 57.52 & 42.48 & 0.270 & 3.700 \\
174 & 56.32 & 43.68 & 0.265 & 3.779 \\
184 & 55.19 & 44.81 & 0.259 & 3.857 \\
194 & 54.06 & 45.94 & 0.254 & 3.937 \\
204 & 53.00 & 47.00 & 0.249 & 4.016 \\
214 & 52.01 & 47.99 & 0.244 & 4.093 \\
224 & 50.22 & 49.78 & 0.236 & 4.239 \\
234 & 49.33 & 50.67 & 0.232 & 4.315 \\
\hline
\end{tabular}


Temperature: $-53^{\circ} \mathrm{C}, 1 \mathrm{~mol} \%$ :

Using General Procedure 3a, a solution of benzaldehyde in chloroform ( $85 \mu \mathrm{L}, 0.35 \mathrm{mmol})$ was injected into an NMR tube containing enolate $4(65 \mu \mathrm{L}, 0.65 \mathrm{mmol})$ and phosphoramide $( \pm)-7 \mathbf{j}$ $(1.7 \mathrm{mg}, 0.004 \mathrm{mmol})$ in methylene chloride- $d_{2}(0.6 \mathrm{~mL})$ at $-53{ }^{\circ} \mathrm{C}$ as internally monitored. The reaction was monitored every 10 seconds until complete conversion. $\mathrm{k}_{\mathrm{obs}}=0.007 \mathrm{M}^{-1} \mathrm{~s}^{-1}$.

\begin{tabular}{|ccccc|}
\hline Time (s) & \% Enolate & \% Aldolate & [Enolate] & 1/[Enolate] \\
\hline \hline 0 & 100.00 & 0.00 & 0.470 & 2.129 \\
4 & - & - & - & - \\
14 & 91.60 & 8.40 & 0.430 & 2.324 \\
24 & 89.15 & 10.85 & 0.419 & 2.388 \\
34 & 86.86 & 13.14 & 0.408 & 2.450 \\
44 & 84.72 & 15.28 & 0.398 & 2.513 \\
54 & 82.65 & 17.35 & 0.388 & 2.575 \\
64 & 80.57 & 19.43 & 0.379 & 2.642 \\
74 & 78.65 & 21.35 & 0.369 & 2.707 \\
84 & 76.81 & 23.19 & 0.361 & 2.771 \\
94 & 75.14 & 24.86 & 0.353 & 2.833 \\
104 & 73.50 & 26.50 & 0.345 & 2.896 \\
114 & 71.98 & 28.02 & 0.338 & 2.957 \\
124 & 70.57 & 29.43 & 0.332 & 3.016 \\
134 & 69.13 & 30.87 & 0.325 & 3.079 \\
144 & 67.84 & 32.16 & 0.319 & 3.138 \\
154 & 66.67 & 33.33 & 0.313 & 3.193 \\
164 & 65.45 & 34.55 & 0.307 & 3.252 \\
174 & 64.30 & 35.70 & 0.302 & 3.310 \\
184 & 63.20 & 36.80 & 0.297 & 3.368 \\
194 & 62.28 & 37.72 & 0.293 & 3.418 \\
204 & 59.39 & 40.61 & 0.279 & 3.584 \\
214 & 58.52 & 41.48 & 0.275 & 3.637 \\
224 & 57.74 & 42.26 & 0.271 & 3.687 \\
234 & 56.91 & 43.09 & 0.267 & 3.740 \\
\hline
\end{tabular}




\section{Temperature: $-53{ }^{\circ} \mathrm{C}, 0.5 \mathrm{~mol} \%$ :}

Using General Procedure 3a, a solution of benzaldehyde in chloroform ( $85 \mu \mathrm{L}, 0.35 \mathrm{mmol})$ was injected into an NMR tube containing enolate $4(65 \mu \mathrm{L}, 0.65 \mathrm{mmol})$ and phosphoramide $( \pm)-7 \mathbf{j}$ $(0.9 \mathrm{mg}, 0.002 \mathrm{mmol})$ in methylene chloride- $d_{2}(0.6 \mathrm{~mL})$ at $-53{ }^{\circ} \mathrm{C}$ as internally monitored. The reaction was monitored every 10 seconds until complete conversion. $\mathrm{k}_{\mathrm{obs}}=0.006 \mathrm{M}^{-1} \mathrm{~s}^{-1}$.

\begin{tabular}{|ccccc|}
\hline Time (s) & \% Enolate & \% Aldolate & [Enolate] & 1/[Enolate] \\
\hline \hline 0 & 100.00 & 0.00 & 0.470 & 2.129 \\
4 & - & - & - & - \\
14 & 95.35 & 4.65 & 0.448 & 2.232 \\
24 & 92.96 & 7.04 & 0.437 & 2.290 \\
34 & 90.65 & 9.35 & 0.426 & 2.348 \\
44 & 88.45 & 11.55 & 0.416 & 2.407 \\
54 & 86.37 & 13.63 & 0.406 & 2.464 \\
64 & 84.43 & 15.57 & 0.397 & 2.521 \\
74 & 82.53 & 17.47 & 0.388 & 2.579 \\
84 & 80.79 & 19.21 & 0.380 & 2.635 \\
94 & 79.04 & 20.96 & 0.371 & 2.693 \\
104 & 77.45 & 22.55 & 0.364 & 2.748 \\
114 & 75.88 & 24.12 & 0.356 & 2.805 \\
124 & 71.10 & 28.90 & 0.334 & 2.994 \\
134 & 69.76 & 30.24 & 0.328 & 3.051 \\
144 & 68.45 & 31.55 & 0.322 & 3.110 \\
154 & 67.16 & 32.84 & 0.316 & 3.169 \\
164 & 65.88 & 34.12 & 0.310 & 3.231 \\
174 & 64.72 & 35.28 & 0.304 & 3.289 \\
184 & 63.59 & 36.41 & 0.299 & 3.347 \\
194 & 62.51 & 37.49 & 0.294 & 3.405 \\
204 & 61.46 & 38.54 & 0.289 & 3.463 \\
214 & 59.49 & 40.51 & 0.279 & 3.578 \\
224 & 61.20 & 38.80 & 0.288 & 3.478 \\
234 & 60.36 & 39.64 & 0.284 & 3.526 \\
\hline
\end{tabular}




\section{Temperature: $-53{ }^{\circ} \mathrm{C}, 0.0 \mathrm{~mol} \%$ :}

Using General Procedure 3a, a solution of benzaldehyde in chloroform ( $85 \mu \mathrm{L}, 0.35 \mathrm{mmol})$ was injected into an NMR tube containing enolate $4(65 \mu \mathrm{L}, 0.65 \mathrm{mmol})$ in methylene chloride- $d_{2}$ $(0.6 \mathrm{~mL})$ at $-53{ }^{\circ} \mathrm{C}$ as internally monitored. The reaction was monitored every 10 seconds for several minutes. $\mathrm{k}=0.001 \mathrm{M}^{-1} \mathrm{~s}^{-1}$.

\begin{tabular}{|ccccc|}
\hline Time $(\mathbf{s})$ & \% Enolate & \% Aldolate & [Enolate] & 1/[Enolate] \\
\hline \hline 0 & 100.00 & 0.00 & 0.437 & 2.289 \\
4 & 97.49 & 2.51 & 0.426 & 2.348 \\
14 & 97.42 & 2.58 & 0.426 & 2.349 \\
24 & 96.97 & 3.03 & 0.424 & 2.360 \\
34 & 96.59 & 3.41 & 0.422 & 2.369 \\
44 & 96.16 & 3.84 & 0.420 & 2.380 \\
54 & 95.81 & 4.19 & 0.419 & 2.389 \\
64 & 95.40 & 4.60 & 0.417 & 2.399 \\
74 & 95.03 & 4.97 & 0.415 & 2.408 \\
84 & 94.64 & 5.36 & 0.414 & 2.418 \\
94 & 94.23 & 5.77 & 0.412 & 2.429 \\
104 & 93.89 & 6.11 & 0.410 & 2.437 \\
114 & 93.50 & 6.50 & 0.409 & 2.448 \\
124 & 93.16 & 6.84 & 0.407 & 2.456 \\
134 & 92.80 & 7.20 & 0.405 & 2.466 \\
144 & 92.40 & 7.60 & 0.404 & 2.477 \\
154 & 92.05 & 7.95 & 0.402 & 2.486 \\
164 & 91.70 & 8.30 & 0.401 & 2.496 \\
174 & 91.36 & 8.64 & 0.399 & 2.505 \\
184 & 91.01 & 8.99 & 0.398 & 2.515 \\
\hline
\end{tabular}

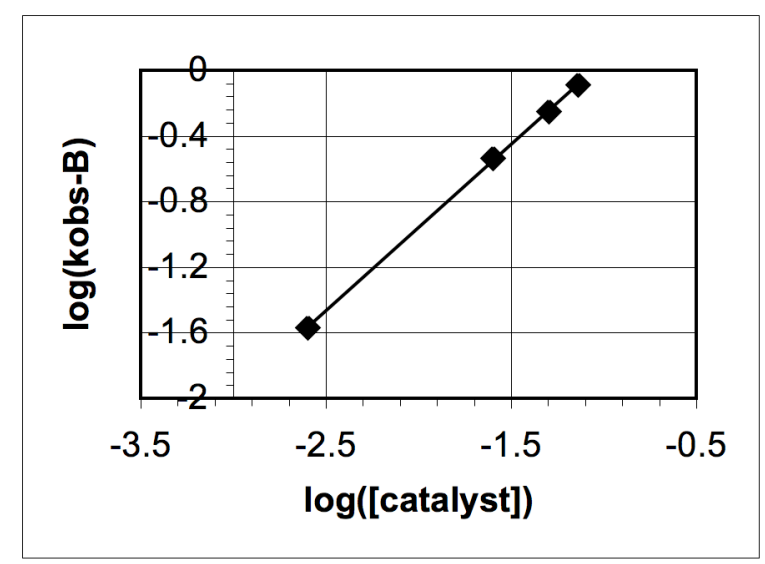

Figure VI. Plot of $\log \left(\mathrm{k}_{\mathrm{obs}}-\mathrm{B}\right.$ versus $\log ([$ catalyst] $)$ for the addition of 4 to benzaldehyde catalyzed by $( \pm)-7 \mathbf{j}$ at $\mathrm{T}=-35^{\circ} \mathrm{C}$. The graph depicts the linear fit to $f(x)=m x+b\left(m=1.01, R^{2}\right.$ $=0.99)$. 


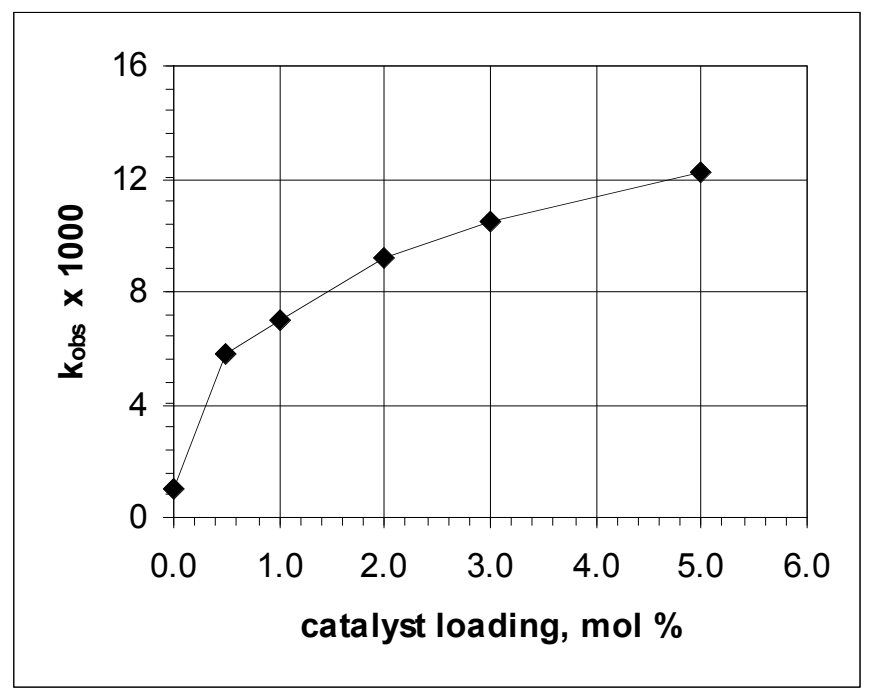

Figure VII. Plot of $\mathrm{k}_{\mathrm{obs}}$ versus catalyst loading for the addition of $\mathbf{4}$ to benzaldehyde catalyzed by $( \pm)-7 \mathbf{j}$. 


\section{Raw Data. RINMR Kinetics of the Addition of 4 to 5a Catalyzed by $(S, S)-7$ a}

\section{Series \#1, Temperature: $-96^{\circ} \mathrm{C}$ :}

Using General Procedure 3b, a solution of benzaldehyde in chloroform ( $44 \mu \mathrm{L}, 0.086 \mathrm{mmol}$ ) was injected into an NMR tube containing enolate $4(16 \mu \mathrm{L}, 0.086 \mathrm{mmol})$ and phosphoramide $(S, S)$ 7a $(3.2 \mathrm{mg}, 0.009 \mathrm{mmol})$ in methylene chloride- $d_{2}(0.6 \mathrm{~mL})$ with a standard solution of TMS in chloroform- $d(0.05 \mathrm{~mL})$ at $-96^{\circ} \mathrm{C}$ as internally monitored. The reaction was monitored every 5 seconds until complete conversion. $\mathrm{k}=48.07 \mathrm{M}^{-2} \mathrm{~s}^{-1}$.

\begin{tabular}{|cccc|}
\hline Time (s) & Integral & [PhCHO] & 1/[PhCHO] \\
\hline \hline 0 & - & 0.124 & 8.069 \\
9 & 1.00 & 0.075 & 13.338 \\
14 & 0.90 & 0.067 & 14.953 \\
19 & 0.81 & 0.060 & 16.603 \\
24 & 0.73 & 0.054 & 18.374 \\
29 & 0.69 & 0.052 & 19.377 \\
34 & 0.65 & 0.049 & 20.603 \\
39 & 0.63 & 0.047 & 21.428 \\
\hline
\end{tabular}

Area of aldehydic proton relative to internal standard

\section{Series \#1, Temperature: $-91^{\circ} \mathrm{C}$ :}

Using General Procedure 3b, a solution of benzaldehyde in chloroform ( $44 \mu \mathrm{L}, 0.086 \mathrm{mmol}$ ) was injected into an NMR tube containing enolate $4(16 \mu \mathrm{L}, 0.086 \mathrm{mmol})$ and phosphoramide $(S, S)$ 7a $(3.2 \mathrm{mg}, 0.009 \mathrm{mmol})$ in methylene chloride- $d_{2}(0.6 \mathrm{~mL})$ with a standard solution of TMS in chloroform- $d(0.05 \mathrm{~mL})$ at $-91{ }^{\circ} \mathrm{C}$ as internally monitored. The reaction was monitored every 5 seconds until complete conversion. $\mathrm{k}=55.68 \mathrm{M}^{-2} \mathrm{~s}^{-1}$.

\begin{tabular}{|cccc|}
\hline Time (s) & Integral & [PhCHO] & 1/[PhCHO] \\
\hline \hline 0 & - & 0.124 & 8.069 \\
9 & 0.90 & 0.067 & 14.883 \\
14 & 0.93 & 0.070 & 14.378 \\
19 & 0.91 & 0.068 & 14.790 \\
24 & 0.86 & 0.065 & 15.493 \\
\hline
\end{tabular}

Area of aldehydic proton relative to internal standard

\section{Series \#1, Temperature: $-85^{\circ} \mathrm{C}$ :}

Using General Procedure 3b, a solution of benzaldehyde in chloroform ( $44 \mu \mathrm{L}, 0.086 \mathrm{mmol}$ ) was injected into an NMR tube containing enolate $4(16 \mu \mathrm{L}, 0.086 \mathrm{mmol})$ and phosphoramide $(S, S)$ $7 \mathbf{a}(3.2 \mathrm{mg}, 0.009 \mathrm{mmol})$ in methylene chloride- $d_{2}(0.6 \mathrm{~mL})$ with a standard solution of TMS in 
chloroform- $d(0.05 \mathrm{~mL})$ at $-85^{\circ} \mathrm{C}$ as internally monitored. The reaction was monitored every 5 seconds until complete conversion. $\mathrm{k}=64.05 \mathrm{M}^{-2} \mathrm{~s}^{-1}$.

\begin{tabular}{|cccc|}
\hline Time (s) & Integral & [PhCHO] & 1/[PhCHO] \\
\hline \hline 0 & - & 0.124 & 8.069 \\
9 & 0.87 & 0.065 & 15.402 \\
14 & 0.75 & 0.056 & 17.957 \\
19 & 0.64 & 0.048 & 20.802 \\
24 & 0.58 & 0.043 & 23.106 \\
29 & 0.53 & 0.040 & 25.244 \\
34 & 0.47 & 0.035 & 28.656 \\
39 & 0.44 & 0.033 & 30.701 \\
\hline
\end{tabular}

Area of aldehydic proton relative to internal standard

\section{Series \#1, Temperature: $\mathbf{- 8 0}{ }^{\circ} \mathbf{C}$ :}

Using General Procedure 3b, a solution of benzaldehyde in chloroform ( $44 \mu \mathrm{L}, 0.086 \mathrm{mmol})$ was injected into an NMR tube containing enolate $4(16 \mu \mathrm{L}, 0.086 \mathrm{mmol})$ and phosphoramide $(S, S)$ $7 \mathbf{a}(3.2 \mathrm{mg}, 0.009 \mathrm{mmol})$ in methylene chloride- $d_{2}(0.6 \mathrm{~mL})$ with a standard solution of TMS in chloroform- $d(0.05 \mathrm{~mL})$ at $-85{ }^{\circ} \mathrm{C}$ as internally monitored. The reaction was monitored every 5 seconds until complete conversion. $\mathrm{k}=70.78 \mathrm{M}^{-2} \mathrm{~s}^{-1}$.

\begin{tabular}{|cccc|}
\hline Time (s) & Integral & [PhCHO] & 1/[PhCHO] \\
\hline 0 & - & 0.124 & 8.069 \\
9 & 0.84 & 0.062 & 16.036 \\
14 & 0.71 & 0.053 & 18.947 \\
19 & 0.61 & 0.045 & 21.996 \\
24 & 0.55 & 0.041 & 24.542 \\
29 & 0.50 & 0.037 & 26.936 \\
34 & 0.44 & 0.033 & 30.580 \\
39 & 0.40 & 0.030 & 33.195 \\
\hline
\end{tabular}

Area of aldehydic proton relative to internal standard

\section{Series \#2, Temperature: $-\mathbf{8 5}{ }^{\circ} \mathrm{C}$ :}

Using General Procedure 3b, a solution of benzaldehyde in chloroform ( $44 \mu \mathrm{L}, 0.086 \mathrm{mmol}$ ) was injected into an NMR tube containing enolate $4(16 \mu \mathrm{L}, 0.086 \mathrm{mmol})$ and phosphoramide $(S, S)$ $7 \mathbf{a}(3.2 \mathrm{mg}, 0.009 \mathrm{mmol})$ in methylene chloride- $d_{2}(0.6 \mathrm{~mL})$ with a standard solution of TMS in chloroform- $d(0.05 \mathrm{~mL})$ at $-85^{\circ} \mathrm{C}$ as internally monitored. The reaction was monitored every 5 seconds until complete conversion. $\mathrm{k}=29.72 \mathrm{M}^{-2} \mathrm{~s}^{-1}$. 


\begin{tabular}{|cccc|}
\hline Time (s) & Integral & [PhCHO] & 1/[PhCHO] \\
\hline 0 & - & 0.124 & 8.069 \\
9 & 1.24 & 0.093 & 10.784 \\
14 & 1.15 & 0.086 & 11.631 \\
19 & 1.12 & 0.083 & 11.999 \\
24 & 1.04 & 0.078 & 12.894 \\
29 & 0.98 & 0.074 & 13.603 \\
34 & 0.93 & 0.070 & 14.369 \\
39 & 0.92 & 0.069 & 14.593 \\
\hline
\end{tabular}

Area of aldehydic proton relative to internal standard

\section{Series \#2, Temperature: $-\mathbf{8 0}{ }^{\circ} \mathrm{C}$ :}

Using General Procedure 3b, a solution of benzaldehyde in chloroform ( $44 \mu \mathrm{L}, 0.086 \mathrm{mmol}$ ) was injected into an NMR tube containing enolate $4(16 \mu \mathrm{L}, 0.086 \mathrm{mmol})$ and phosphoramide $(S, S)$ $7 \mathbf{a}(3.2 \mathrm{mg}, 0.009 \mathrm{mmol})$ in methylene chloride- $d_{2}(0.6 \mathrm{~mL})$ with a standard solution of TMS in chloroform- $d(0.05 \mathrm{~mL})$ at $-80{ }^{\circ} \mathrm{C}$ as internally monitored. The reaction was monitored every 5 seconds until complete conversion. $\mathrm{k}=36.24 \mathrm{M}^{-2} \mathrm{~s}^{-1}$.

\begin{tabular}{|cccc|}
\hline Time (s) & Integral & [PhCHO] & 1/[PhCHO] \\
\hline \hline 0 & - & 0.124 & 8.069 \\
5 & 1.02 & 0.076 & 13.169 \\
10 & 1.02 & 0.076 & 13.074 \\
15 & 1.00 & 0.075 & 13.385 \\
20 & 0.98 & 0.073 & 13.720 \\
25 & 0.95 & 0.071 & 14.105 \\
30 & 0.92 & 0.069 & 14.583 \\
35 & 0.89 & 0.067 & 14.970 \\
\hline
\end{tabular}

\section{Series \#2, Temperature: $-75^{\circ} \mathrm{C}$ :}

Using General Procedure 3b, a solution of benzaldehyde in chloroform ( $44 \mu \mathrm{L}, 0.086 \mathrm{mmol}$ ) was injected into an NMR tube containing enolate $4(16 \mu \mathrm{L}, 0.086 \mathrm{mmol})$ and phosphoramide $(S, S)$ 7a $(3.2 \mathrm{mg}, 0.009 \mathrm{mmol})$ in methylene chloride- $d_{2}(0.6 \mathrm{~mL})$ with a standard solution of TMS in chloroform- $d(0.05 \mathrm{~mL})$ at $-75^{\circ} \mathrm{C}$ as internally monitored. The reaction was monitored every 5 seconds until complete conversion. $\mathrm{k}=44.06 \mathrm{M}^{-2} \mathrm{~s}^{-1}$. 


\begin{tabular}{|c|c|c|c|}
\hline Time (s) & Integral $^{\dagger}$ & [PhCHO] & 1/[PhCHO] \\
\hline 0 & - & 0.124 & 8.069 \\
\hline 9 & 1.10 & 0.082 & 12.194 \\
\hline 14 & 0.94 & 0.070 & 14.236 \\
\hline 19 & 0.85 & 0.063 & 15.831 \\
\hline 24 & 0.77 & 0.057 & 17.441 \\
\hline 29 & 0.68 & 0.051 & 19.674 \\
\hline 34 & 0.62 & 0.046 & 21.759 \\
\hline 39 & 0.58 & 0.043 & 23.262 \\
\hline
\end{tabular}

Area of aldehydic proton relative to internal standard

\section{Series \#3, Temperature: $-\mathbf{7 0}^{\circ} \mathrm{C}$ :}

Using General Procedure 3b, a solution of benzaldehyde in chloroform ( $44 \mu \mathrm{L}, 0.086 \mathrm{mmol}$ ) was injected into an NMR tube containing enolate $4(16 \mu \mathrm{L}, 0.086 \mathrm{mmol})$ and phosphoramide $(S, S)$ $7 \mathbf{a}(3.2 \mathrm{mg}, 0.009 \mathrm{mmol})$ in methylene chloride- $d_{2}(0.6 \mathrm{~mL})$ with a standard solution of TMS in chloroform- $d(0.05 \mathrm{~mL})$ at $-70{ }^{\circ} \mathrm{C}$ as internally monitored. The reaction was monitored every 5 seconds until complete conversion. $\mathrm{k}=56.46 \mathrm{M}^{-2} \mathrm{~s}^{-1}$.

\begin{tabular}{|cccc|}
\hline Time (s) & Integral $^{\dagger}$ & [PhCHO] $^{\text {[Ph }}$ & 1/[PhCHO] \\
\hline 0 & - & 0.124 & 8.069 \\
9 & 1.08 & 0.081 & 12.390 \\
14 & 1.00 & 0.075 & 13.362 \\
19 & 0.94 & 0.070 & 14.254 \\
24 & 0.86 & 0.064 & 15.511 \\
29 & 0.80 & 0.060 & 16.688 \\
34 & 0.76 & 0.057 & 17.515 \\
39 & 0.73 & 0.055 & 18.323 \\
\hline
\end{tabular}

Area of aldehydic proton relative to internal standard

\section{Series \#3, Temperature: $-75^{\circ} \mathrm{C}$ :}

Using General Procedure 3b, a solution of benzaldehyde in chloroform ( $44 \mu \mathrm{L}, 0.086 \mathrm{mmol}$ ) was injected into an NMR tube containing enolate $4(16 \mu \mathrm{L}, 0.086 \mathrm{mmol})$ and phosphoramide $(S, S)$ $7 \mathbf{a}(3.2 \mathrm{mg}, 0.009 \mathrm{mmol})$ in methylene chloride- $d_{2}(0.6 \mathrm{~mL})$ with a standard solution of TMS in chloroform- $d(0.05 \mathrm{~mL})$ at $-75^{\circ} \mathrm{C}$ as internally monitored. The reaction was monitored every 5 seconds until complete conversion. $\mathrm{k}=46.53 \mathrm{M}^{-2} \mathrm{~s}^{-1}$. 


\begin{tabular}{|c|c|c|c|}
\hline Time (s) & Integral $^{\dagger}$ & [PhCHO] & 1/[PhCHO] \\
\hline 0 & - & 0.124 & 8.069 \\
\hline 9 & 1.30 & 0.097 & 10.336 \\
\hline 14 & 1.20 & 0.089 & 11.181 \\
\hline 19 & 1.13 & 0.084 & 11.904 \\
\hline 24 & 1.05 & 0.079 & 12.709 \\
\hline 29 & 0.99 & 0.074 & 13.497 \\
\hline 34 & 0.95 & 0.071 & 14.142 \\
\hline 39 & 0.90 & 0.067 & 14.842 \\
\hline
\end{tabular}

Area of aldehydic proton relative to internal standard

\section{Series \#3, Temperature: $-75^{\circ} \mathrm{C}$ :}

Using General Procedure 3b, a solution of benzaldehyde in chloroform ( $44 \mu \mathrm{L}, 0.086 \mathrm{mmol}$ ) was injected into an NMR tube containing enolate $4(16 \mu \mathrm{L}, 0.086 \mathrm{mmol})$ and phosphoramide $(S, S)$ $7 \mathbf{a}(3.2 \mathrm{mg}, 0.009 \mathrm{mmol})$ in methylene chloride- $d_{2}(0.6 \mathrm{~mL})$ with a standard solution of TMS in chloroform- $d(0.05 \mathrm{~mL})$ at $-75^{\circ} \mathrm{C}$ as internally monitored. The reaction was monitored every 5 seconds until complete conversion. $\mathrm{k}=16.47 \mathrm{M}^{-2} \mathrm{~s}^{-1}$.

\begin{tabular}{|cccc|}
\hline Time (s) & Integral $^{\dagger}$ & [PhCHO] $^{\text {1/[PhCHO] }}$ \\
\hline 0 & - & 0.124 & 8.069 \\
9 & 1.15 & 0.086 & 11.636 \\
14 & 1.17 & 0.087 & 11.472 \\
19 & 1.14 & 0.085 & 11.779 \\
24 & 1.14 & 0.085 & 11.785 \\
29 & 1.12 & 0.083 & 11.978 \\
\hline
\end{tabular}

Area of aldehydic proton relative to internal standard

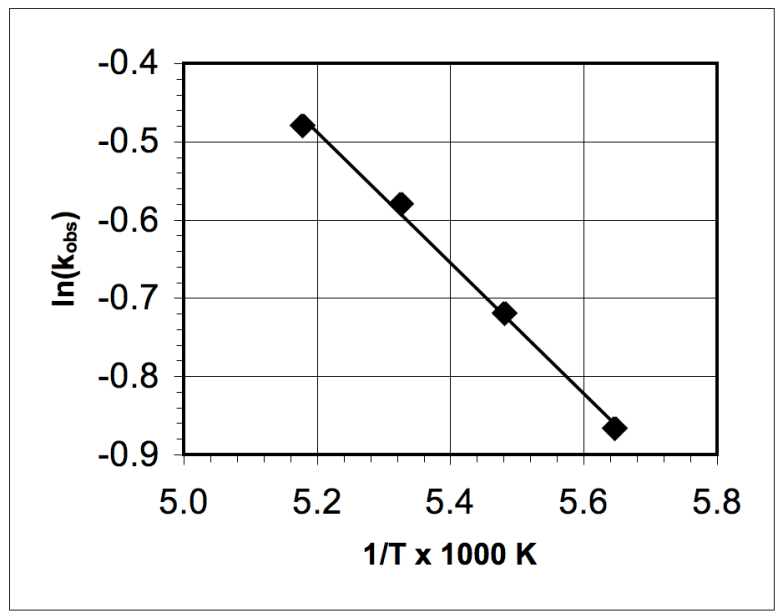

Figure VIII. Arrhenius Plot. The graph depicts a linear fit, $f(x)=m x+b\left(m=-0.84, R^{2}=1.00\right)$. 


\section{Raw Data. RINMR Loading Studies on the Addition of 4 to 5a Catalyzed by $(S, S)-7$ a, Temperature: $-80^{\circ} \mathrm{C}, 10 \mathrm{~mol} \%$ :}

Using General Procedure 3b, a solution of benzaldehyde in chloroform ( $44 \mu \mathrm{L}, 0.086 \mathrm{mmol}$ ) was injected into an NMR tube containing enolate $4(16 \mu \mathrm{L}, 0.086 \mathrm{mmol})$ and phosphoramide $(S, S)$ $7 \mathbf{a}(3.2 \mathrm{mg}, 0.009 \mathrm{mmol})$ in methylene chloride- $d_{2}(0.6 \mathrm{~mL})$ with a standard solution of TMS in chloroform- $d(0.05 \mathrm{~mL})$ at $-80{ }^{\circ} \mathrm{C}$ as internally monitored. The reaction was monitored every 2 seconds until complete conversion. $\mathrm{k}_{\mathrm{obs}}=0.513 \mathrm{M}^{-1} \mathrm{~s}^{-1}$.

\begin{tabular}{|cccc|}
\hline Time (s) & Integral & [PhCHO] & 1/[PhCHO] \\
\hline \hline 0 & - & 0.124 & 8.069 \\
5 & 1.20 & 0.071 & 14.041 \\
7 & 1.16 & 0.069 & 14.526 \\
9 & 1.12 & 0.066 & 15.103 \\
11 & 1.06 & 0.063 & 15.835 \\
13 & 1.03 & 0.061 & 16.388 \\
15 & 0.97 & 0.058 & 17.303 \\
17 & 0.94 & 0.056 & 17.887 \\
\hline
\end{tabular}

Area of aldehydic proton relative to internal standard

\section{Temperature: $-80{ }^{\circ} \mathrm{C}, 13 \mathrm{~mol} \%$ :}

Using General Procedure 3b, a solution of benzaldehyde in chloroform ( $44 \mu \mathrm{L}, 0.086 \mathrm{mmol}$ ) was injected into an NMR tube containing enolate $4(16 \mu \mathrm{L}, 0.086 \mathrm{mmol})$ and phosphoramide $(S, S)$ $7 \mathbf{a}(4.1 \mathrm{mg}, 0.011 \mathrm{mmol})$ in methylene chloride- $d_{2}(0.6 \mathrm{~mL})$ with a standard solution of TMS in chloroform- $d(0.05 \mathrm{~mL})$ at $-80{ }^{\circ} \mathrm{C}$ as internally monitored. The reaction was monitored every 1 second until complete conversion. $\mathrm{k}_{\mathrm{obs}}=0.810 \mathrm{M}^{-1} \mathrm{~s}^{-1}$.

\begin{tabular}{|cccc|}
\hline Time (s) & Integral & [PhCHO] & 1/[PhCHO] \\
\hline 0 & - & 0.124 & 8.069 \\
5 & 1.11 & 0.083 & 12.085 \\
6 & 1.03 & 0.077 & 12.944 \\
7 & 1.00 & 0.074 & 13.442 \\
8 & 0.95 & 0.071 & 14.071 \\
9 & 0.92 & 0.069 & 14.506 \\
10 & 0.87 & 0.065 & 15.334 \\
\hline
\end{tabular}

Area of aldehydic proton relative to internal standard

\section{Temperature: $-80{ }^{\circ} \mathrm{C}, 15 \mathrm{~mol} \%$ :}

Using General Procedure 3b, a solution of benzaldehyde in chloroform ( $44 \mu \mathrm{L}, 0.086 \mathrm{mmol}$ ) was injected into an NMR tube containing enolate $4(16 \mu \mathrm{L}, 0.086 \mathrm{mmol})$ and phosphoramide $(S, S)$ - 
$7 \mathbf{a}(4.8 \mathrm{mg}, 0.013 \mathrm{mmol})$ in methylene chloride- $d_{2}(0.6 \mathrm{~mL})$ with a standard solution of TMS in chloroform- $d(0.05 \mathrm{~mL})$ at $-80{ }^{\circ} \mathrm{C}$ as internally monitored. The reaction was monitored every 1 second until complete conversion. $\mathrm{k}_{\mathrm{obs}}=1.133 \mathrm{M}^{-1} \mathrm{~s}^{-1}$.

\begin{tabular}{|cccc|}
\hline Time (s) & Integral & [PhCHO] & $\mathbf{1 / [ P h C H O ] ~}$ \\
\hline 0 & - & 0.124 & 8.069 \\
5 & 1.02 & 0.076 & 13.156 \\
6 & 0.88 & 0.066 & 15.195 \\
7 & 0.80 & 0.059 & 16.846 \\
8 & 0.69 & 0.052 & 19.402 \\
9 & 0.65 & 0.048 & 20.711 \\
10 & 0.59 & 0.044 & 22.756 \\
\hline
\end{tabular}

Area of aldehydic proton relative to internal standard

\section{Temperature: $-80{ }^{\circ} \mathrm{C}, 16 \mathrm{~mol} \%$ :}

Using General Procedure 3b, a solution of benzaldehyde in chloroform ( $44 \mu \mathrm{L}, 0.086 \mathrm{mmol}$ ) was injected into an NMR tube containing enolate $4(16 \mu \mathrm{L}, 0.086 \mathrm{mmol})$ and phosphoramide $(S, S)$ $7 \mathbf{a}(5.2 \mathrm{mg}, 0.014 \mathrm{mmol})$ in methylene chloride- $d_{2}(0.6 \mathrm{~mL})$ with a standard solution of TMS in chloroform- $d(0.05 \mathrm{~mL})$ at $-80{ }^{\circ} \mathrm{C}$ as internally monitored. The reaction was monitored every 1 second until complete conversion. $\mathrm{k}_{\mathrm{obs}}=1.314 \mathrm{M}^{-1} \mathrm{~s}^{-1}$.

\begin{tabular}{|cccc|}
\hline Time (s) & Integral & [PhCHO] & 1/[PhCHO] \\
\hline 0 & - & 0.124 & 8.069 \\
5 & 1.08 & 0.081 & 12.409 \\
6 & 0.92 & 0.069 & 14.513 \\
7 & 0.79 & 0.059 & 17.021 \\
8 & 0.71 & 0.053 & 18.991 \\
9 & 0.60 & 0.045 & 22.250 \\
10 & 0.54 & 0.040 & 25.019 \\
\hline
\end{tabular}

Area of aldehydic proton relative to internal standard

\section{Temperature: $-80^{\circ} \mathrm{C}, 17 \mathrm{~mol} \%$ :}

Using General Procedure 3b, a solution of benzaldehyde in chloroform ( $44 \mu \mathrm{L}, 0.086 \mathrm{mmol}$ ) was injected into an NMR tube containing enolate $4(16 \mu \mathrm{L}, 0.086 \mathrm{mmol})$ and phosphoramide $(S, S)$ $7 \mathbf{a}(5.5 \mathrm{mg}, 0.015 \mathrm{mmol})$ in methylene chloride- $d_{2}(0.6 \mathrm{~mL})$ with a standard solution of TMS in chloroform- $d(0.05 \mathrm{~mL})$ at $-80^{\circ} \mathrm{C}$ as internally monitored. The reaction was monitored every 1 second until complete conversion. $\mathrm{k}_{\mathrm{obs}}=1.502 \mathrm{M}^{-1} \mathrm{~s}^{-1}$. 


\begin{tabular}{|cccc|}
\hline Time (s) & Integral & [PhCHO] & 1/[PhCHO] \\
\hline 0 & - & 0.124 & 8.069 \\
5 & 0.97 & 0.072 & 13.854 \\
6 & 0.81 & 0.061 & 16.484 \\
7 & 0.69 & 0.052 & 19.281 \\
8 & 0.61 & 0.045 & 22.129 \\
9 & 0.54 & 0.040 & 24.817 \\
10 & 0.46 & 0.035 & 28.944 \\
\hline
\end{tabular}

Area of aldehydic proton relative to internal standard

\section{Temperature: $-80{ }^{\circ} \mathrm{C}, 18 \mathrm{~mol} \%$ :}

Using General Procedure 3b, a solution of benzaldehyde in chloroform ( $44 \mu \mathrm{L}, 0.086 \mathrm{mmol}$ ) was injected into an NMR tube containing enolate $4(16 \mu \mathrm{L}, 0.086 \mathrm{mmol})$ and phosphoramide $(S, S)$ $7 \mathbf{a}(5.9 \mathrm{mg}, 0.016 \mathrm{mmol})$ in methylene chloride- $d_{2}(0.6 \mathrm{~mL})$ with a standard solution of TMS in chloroform- $d(0.05 \mathrm{~mL})$ at $-80{ }^{\circ} \mathrm{C}$ as internally monitored. The reaction was monitored every 1 second until complete conversion. $\mathrm{k}_{\mathrm{obs}}=1.803 \mathrm{M}^{-1} \mathrm{~s}^{-1}$.

\begin{tabular}{|cccc|}
\hline Time (s) & Integral & [PhCHO] & 1/[PhCHO] \\
\hline 0 & - & 0.124 & 8.069 \\
5 & 1.05 & 0.078 & 12.757 \\
6 & 0.85 & 0.064 & 15.722 \\
7 & 0.72 & 0.054 & 18.570 \\
8 & 0.63 & 0.047 & 21.312 \\
9 & 0.54 & 0.040 & 24.916 \\
10 & 0.48 & 0.036 & 27.915 \\
\hline
\end{tabular}

Area of aldehydic proton relative to internal standard

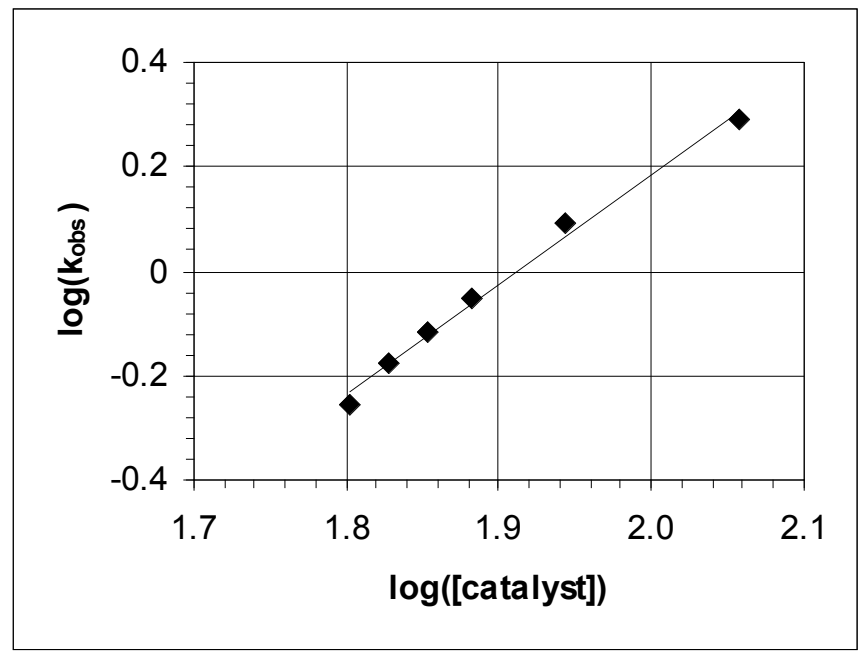

Figure IX. Plot of $\log \left(\mathrm{k}_{\mathrm{obs}}\right)$ versus $\log ([$ catalyst $])$ for the addition of $\mathbf{4}$ to benzaldehyde catalyzed by $(S, S)-7 \mathbf{a}$ at $\mathrm{T}=-80{ }^{\circ} \mathrm{C}$. The graph depicts the linear fit to $f(x)=m x+b\left(m=2.11, R^{2}=0.99\right)$. 
Natural Abundance ${ }^{13} \mathrm{C}$ NMR Kinetic Isotope Effects Studies

\section{Limiting Aldehyde}

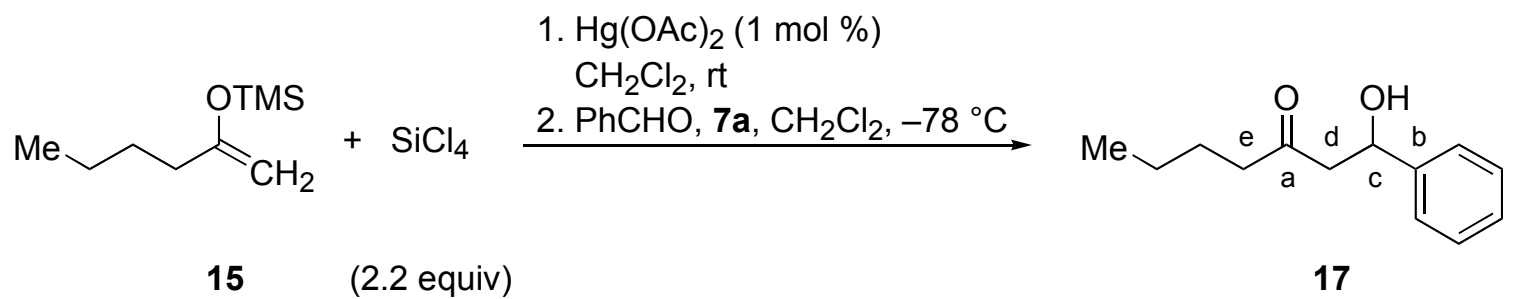

Silyl enol ether 15 (862 $\mathrm{mg}, 5.0 \mathrm{mmol})$ was added quickly to a stirred suspension of silicon tetrachloride $(1.1 \mathrm{~mL}, 10.0 \mathrm{mmol}, 2.0$ equiv) and mercuric acetate (16 mg, $0.05 \mathrm{mmol}$, 0.01 equiv) in $\mathrm{CH}_{2} \mathrm{Cl}_{2}(5 \mathrm{~mL})$ at room temperature. After addition, the mixture was stirred at room temperature for $2 \mathrm{~h}$. The volatile components were then removed under reduced pressure $(0.1 \mathrm{mmHg})$ to give a cloudy oil. A solution of catalyst $7 \mathbf{a}(184 \mathrm{mg}, 0.5 \mathrm{mmol}, 0.10$ equiv) in $\mathrm{CH}_{2} \mathrm{Cl}_{2}(10 \mathrm{~mL})$ was then added via cannula and the mixture was cooled to $-78{ }^{\circ} \mathrm{C}$. Benzaldehyde $(25 \mu \mathrm{L}, 0.25 \mathrm{mmol}, 0.05$ equiv) was then added dropwise via syringe and the reaction mixture was allowed to stir at $-78^{\circ} \mathrm{C}$ for $6 \mathrm{~h}$. The reaction mixture was then poured into a rapidly stirring sat. aq. $\mathrm{NaHCO}_{3}$ solution $(30 \mathrm{~mL})$ submerged in an ice bath and was allowed to stir at room temperature for $6 \mathrm{~h}$. The heterogeneous mixture was then filtered through Celite, the organic phase was separated and the aqueous phase was extracted with $\mathrm{CH}_{2} \mathrm{Cl}_{2}(3 \times$ $10 \mathrm{~mL}$ ). The organic extracts were combined, dried over $\mathrm{Na}_{2} \mathrm{SO}_{4}$, filtered and concentrated to give a crude oil. Purification by silica gel chromatography $\left(\mathrm{SiO}_{2}\right.$, pentane/Et $\left.2 \mathrm{O}, 6 / 1\right)$ afforded 35 mg (95\%) of $\mathbf{1 7}$ as a clear, colorless oil. Parameters for ${ }^{13} \mathrm{C}$ NMR analysis are as follows: $\mathrm{T} 1=6$ $\mathrm{s} ;$ pw90 $=8$. Spectra suitable for integration were obtained using a $\mathrm{d} 1=8 \times \mathrm{T} 1$ and a final signal-to-noise exceeding 250:1.

\begin{tabular}{|c|c|c|c|c|c|c|c|c|c|c|}
\hline Peak & $\delta$ & Run 1 & Run 2 & Run 3 & Run 4 & Run 5 & Average & Standard & Ratio & KIE \\
\hline \hline $\mathrm{a}$ & 211.74 & 1.004 & 1.005 & 1.005 & 1.000 & 1.003 & 1.003 & 1.003 & 1.000 & 1.000 \\
\hline $\mathrm{b}$ & 142.82 & 1.000 & 1.000 & 1.000 & 1.000 & 1.000 & 1.000 & 1.000 & 1.000 & 1.000 \\
\hline $\mathrm{c}$ & 69.89 & 1.988 & 1.992 & 1.987 & 1.979 & 1.993 & 1.988 & 1.988 & 1.000 & 0.997 \\
\hline $\mathrm{d}$ & 50.94 & 1.927 & 1.927 & 1.926 & 1.920 & 1.926 & 1.925 & 1.929 & 0.998 & 0.963 \\
\hline $\mathrm{e}$ & 43.37 & 1.886 & 1.889 & 1.890 & 1.878 & 1.889 & 1.886 & 1.886 & 1.000 & 1.003 \\
\hline
\end{tabular}




\section{Limiting Enolate}

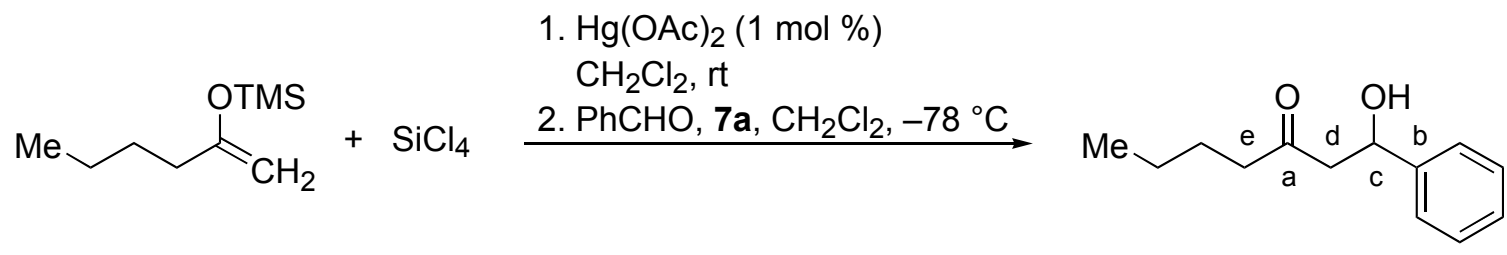

15 (2.2 equiv)

17

Silyl enol ether 15 (86 mg, $0.50 \mathrm{mmol}, 0.05$ equiv) was added quickly to a stirred suspension of silicon tetrachloride $(115 \mu \mathrm{L}, 1.0 \mathrm{mmol}, 0.10$ equiv) and mercuric acetate (1.6 mg, $0.005 \mathrm{mmol}, 0.0005$ equiv) in $\mathrm{CH}_{2} \mathrm{Cl}_{2}(0.5 \mathrm{~mL})$ at room temperature. After addition, the mixture was stirred at room temperature for $2 \mathrm{~h}$. The volatile components were then removed under reduced pressure $(0.1 \mathrm{mmHg})$ to give a cloudy oil. A solution of 7a $(368 \mathrm{mg}, 1.0 \mathrm{mmol}, 0.10$ equiv) in $\mathrm{CH}_{2} \mathrm{Cl}_{2}(10 \mathrm{~mL})$ was then added via cannula and the mixture was cooled to $-78{ }^{\circ} \mathrm{C}$. Benzaldehyde $(1.0 \mathrm{~mL}, 10.0 \mathrm{mmol})$ was then added dropwise via syringe and the reaction mixture was allowed to stir at $-78{ }^{\circ} \mathrm{C}$ for $10 \mathrm{~h}$. The reaction mixture was then poured into a rapidly stirring sat. aq. $\mathrm{NaHCO}_{3}$ solution $(30 \mathrm{~mL})$ submerged in an ice bath and was allowed to stir at room temperature for $6 \mathrm{~h}$. The heterogeneous mixture was then filtered through Celite, the organic phase was separated and the aqueous phase was extracted with $\mathrm{CH}_{2} \mathrm{Cl}_{2}(3 \times 10 \mathrm{~mL})$. The organic extracts were combined, dried over $\mathrm{Na}_{2} \mathrm{SO}_{4}$, filtered and concentrated to give a crude oil. Purification by silica gel chromatography $\left(\mathrm{SiO}_{2}\right.$, pentane/Et $\left.{ }_{2} \mathrm{O}, 6 / 1\right)$ afforded $68 \mathrm{mg}(92 \%)$ of 17 as a clear, colorless oil. Parameters for ${ }^{13} \mathrm{C}$ NMR analysis are as follows: $\mathrm{T} 1=6 \mathrm{~s}$; pw90 $=8$. Spectra suitable for integration were obtained using a $\mathrm{d} 1=8 \times \mathrm{T} 1$ and a final signal-to-noise exceeding 250:1.

\begin{tabular}{|c|c|c|c|c|c|c|c|c|c|c|}
\hline Peak & $\delta$ & Run 1 & Run 2 & Run 3 & Run 4 & Run 5 & Average & Standard & Ratio & KIE \\
\hline \hline $\mathrm{a}$ & 211.74 & 1.005 & 1.002 & 1.002 & 1.004 & 1.004 & 1.003 & 1.003 & 1.000 & 1.002 \\
\hline $\mathrm{b}$ & 142.82 & 1.000 & 1.000 & 1.000 & 1.000 & 1.000 & 1.000 & 1.000 & 1.000 & 1.000 \\
\hline $\mathrm{c}$ & 69.89 & 1.995 & 1.984 & 1.979 & 1.982 & 1.985 & 1.985 & 1.988 & 0.998 & 0.969 \\
\hline $\mathrm{d}$ & 50.94 & 1.931 & 1.930 & 1.925 & 1.928 & 1.930 & 1.929 & 1.929 & 1.000 & 0.995 \\
\hline $\mathrm{e}$ & 43.37 & 1.894 & 1.885 & 1.881 & 1.884 & 1.886 & 1.886 & 1.886 & 1.000 & 1.001 \\
\hline
\end{tabular}




\section{References}

(1) Pikul, S.; Corey, E. J. Org. Syn. 1992, 71, 22.

(2) Denmark, S. E.; Su, X.; Nishigaichi, Y.; Wong, K.-T.; Coe, D. M.; Winter S. B. D.; Choi, J. Y. J. Org. Chem. 1999, 64, 1958-1967.

(3) Yamashita, J.; Tomiyama, S.; Hashimoto, S.; Kitahara, K.; Sato, H. Chem. Lett. 1984, 749 .

(4) (a) Kanemasa, S.; Hayashi, T.; Tanaka, J.; Yamamoto, H.; Sakurai, T. J. Org. CHem. 1991, 56, 4473. (b) Stuhmer, W.; Messwarb, G. Arch. Pharm. 1953, 286, 221 (Chem. Abstr. 1955, 49, 6192b).

(5) Michaelis, A. Liebigs Ann. Chem. 1903, 326, 129.

(6) Otto, P. Ber. 1895, 28, 613.

(7) Mangeney, P.; Grojean, F.; Alexakis, A.; Normant, J.F. Tetrahedron Lett. 1988, 29, 2675.

(8) Russell, G.B.; Sutherland, G.J.; Topsom, R.D.; Vaughan, J. J. Org. Chem. 1962, 27, 4375. 